\title{
Attentional correlates of schizophrenia and related disorders : in search for objective indicators of psychopathology
}

Citation for published version (APA):

van den Bosch, R. J. (1982). Attentional correlates of schizophrenia and related disorders : in search for objective indicators of psychopathology. [Doctoral Thesis, Maastricht University]. Swets \& Zeitlinger. https://doi.org/10.26481/dis.19820903rb

Document status and date:

Published: 01/01/1982

DOI:

10.26481/dis.19820903rb

Document Version:

Publisher's PDF, also known as Version of record

Please check the document version of this publication:

- A submitted manuscript is the version of the article upon submission and before peer-review. There can be important differences between the submitted version and the official published version of record.

People interested in the research are advised to contact the author for the final version of the publication, or visit the DOI to the publisher's website.

- The final author version and the galley proof are versions of the publication after peer review.

- The final published version features the final layout of the paper including the volume, issue and page numbers.

Link to publication

\footnotetext{
General rights rights.

- You may freely distribute the URL identifying the publication in the public portal. please follow below link for the End User Agreement:

www.umlib.nl/taverne-license

Take down policy

If you believe that this document breaches copyright please contact us at:

repository@maastrichtuniversity.nl

providing details and we will investigate your claim.
}

Copyright and moral rights for the publications made accessible in the public portal are retained by the authors and/or other copyright owners and it is a condition of accessing publications that users recognise and abide by the legal requirements associated with these

- Users may download and print one copy of any publication from the public portal for the purpose of private study or research.

- You may not further distribute the material or use it for any profit-making activity or commercial gain

If the publication is distributed under the terms of Article $25 \mathrm{fa}$ of the Dutch Copyright Act, indicated by the "Taverne" license above, 


\section{ATTENTIONAL CORRELATES OF SCHIZOPHRENIA AND RELATED DISORDERS}

In search for objective indicators of psychopathology 


\section{ATTENTIONAL CORRELATES OF SCHIZOPHRENIA AND RELATED DISORDERS}

In search for objective indicators of psychopathology

\section{Proefschrift}

Ter verkrijging van de graad van doctor in de geneeskunde aan de Rijksuniversiteit Limburg te Maastricht, op gezag van de Rector Magnificus Prof. Dr. H. C. Hemker, volgens besluit van het College van Dekanen in het openbaar te verdedigen in de aula van de universiteit op vrijdag 3 september 1982 des namiddags te vier uur.

door

Robert Jan van den Bosch geboren te Boskoop

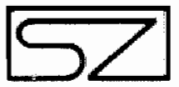

SWETS \& ZEITLINGER B.V. LISSE - THE NETHERLANDS - 1982 
Promotores: Prof. Dr. J. M. F. A, Mol

Prof. Dr. J. J. C. B. Bremer

Referenten: Prof. Dr. A. P. W. M. Appels

Prof. Dr. S. J. Nijdam

Cover H. Veltman

Printed in the Netherlands by Offsetdrukkerij Kanters B.V., Alblasserdam Copyright 1982 R. van den Bosch and Swets \& Zeitlinger B.V.

Alle rechten woorbehouden. Niets uit deze uitgave mag worden verveelvoudigd, opgeslagen in een geautomatiseerd gegevensbestand, of openbaar gemaakt, in enige vorm of op enige wijze, hetzij elektronisch, mechanisch door fotokopieien, opnamen, of op enige andere manier, zonder woorafgaande schriftelijke toestemming wan de uitgever.

All rights reserved. No parts of this publication may be reproduced, stored in a retrieval system, or transmitted, in any form or by any means, electronic, mechanical photocopying. recording, or atherwise, withowt the prior written permission of the publisher. 
To those who

"toically move quietly in the world, asking for little, expecting the worst, and langing for human dignity and equality'.

(Schizophrenia Bulletin, 6: $544-546$ ) 
We humans also have "software' and 'hardware" aspects, and the difference is second nature to us. We are used to the rigidity of our physiology: the fact that we cannot, at will, cure ourselves of diseases, or grow hair of any color - to mention just a couple of simple examples. We can, however, 'reprogran' our minds so that we operate in new conceptual frameworks. The amazing flexibility of our minds seems nearly irreconcilable with the notion that our brains must be made out of fixed-rule hardware, which cannot be reprogrammed. We cannot make our neurons fire faster or slower, we cannot rewire our brains, we cannot redesign the interior of a neuron, we cannot make any choices about the hardware - and yet, we can control how we think.

But there are clearly aspects of thought which are beyond our control. We cannot make ourselves smarter by an act of will, we cannot learn a new language as fast as we want; we cannot make ourselves think faster than we do; we cannot make ourselves think about several things at once; and so on. This is a kind of primordial self-knowledge which is so obvious that it is hard' to see it at all; it is like being conscious that the air is there. We never really bother to think about what might cause these 'defects' of our minds: namely, the organization of our brains.

Douglas $R$. Hofstadter Gotdel, Escher, Bach: An Eternal Golden Braid. 


\section{ACKNOWLEDGEMENTS}

This dissertation is the result of a sustained attention task lasting several years, and characterized by a lot of distraction as vell as reinforcement. I should like to thank the friends and colleagues who have helped me in the preparation of this book. Professor J.M.F.A. Mol has helped me very much and I like to thank him for his encouragement and advice. Professor J.J.C.B. Bremer made many helpful comments and I an grateful for his careful reading of the manuscript. Methodological and statistical advice was excellently provided by professor A.P.W.M. Appels and I am very grateful for his interest and advice. Professor 5.J. Nijdam also read the manuscript and made helpful comments.

I am much indebted to the Stichting Psychisch Gezondheidscentrum Welterhof in Heerlen (director: H.J. Kreutzkamp), which has provided the facilities for my research program. My debts go in particular to the staff of the Department of Clinical Neurophysiology: Marlies Kempers, Lucy Thorissen, and most especially Nico Rozendaal. Without his enthusiasm and energy this work could not have been done. I am also grateful to Jan van Houtem who provided technical assistance at the level of computerized statistical analysis. I would also like to thank my colleagues at my clinical department. I appreciate their support and their readiness to accept my necessarily selective interests in the course of my investigation. My secretary Linda Flekken-Lange has carefully typed several versions of the manuscript and $I$ owe special thanks to her. Finally my gratitude goes to the 
patients and the non-patient volunteers for their willingness to participate in the investigation.

A few people deserve special mention. My parents have always offered me the opportunities to develop my interests, and they did much more. My wife Gonnie and my daughters Mathilde and Simone have allowed my concerns to take precedence of many of their primary interests, for this book was mainly uritten during hours I should have devoted to them. I warmly appreciate their tolerance, generosity and understanding.

vdB. 


\section{CONTENTS}

1. INTRODUCTION.

2. DISORDERS OF ATTENTION AND INFORMATION PROCESSING IN SCHIZOPHRENIA.

2.1. The concept of schizophrenia. 5

2.1.1. History. 5

2.1.2. Etiology and course. 9

2.1.3. Diagnostic criteria. 15

2.2. The concept of attention. 38

2.3. General aspects of attentional dysfunctioning in schizophrenia.

2.4. Models of schizophrenia based on defects of attention and information processing. 52

3. AIMS OF THE INVESTIGATION. 63

4. ASSUMPTIONS AND HYPOTHESES. 67

5. INSTRUMENTS AND METHODS.

5.1. Diagnostic descriptions. $\quad 75$

5.1.1. Subject classification: psychiatric djagnosis. 75

5.1.2. Dimensional descriptions, related to the schizophrenia concept. 79

5.1.3. Dimensional personality description: psychoticism.

5.2. Attentional tasks. 88

5.2.1. Smooth Pursuit Eye Movements. 88

5.2.2. Reaction Time: Simple Mean Reaction Time and Reaction Time Redundancy Deficit (Crossover Phenomenon).

5.2.3. Continuous Performance Test. 114

5.2.4. Contingent Negative Variation. 122 
6. DESIGN AND PROCEDURES. 137

6.1. General design. 137

6.2. Subjects: Characteristics and classification. 138

6.3. Procedures: Attentional tasks. 145

7. RESLLTS. 153

7.1. Testing of hypotheses. 154

7.1.1. Hypothesis I: intercorrelations of attentional variables. 154

7.1.2. Hypotheses II and III: intercorrelations of diagnostic variables and psychiatric diagnosis.

7.1.3. Hypothesis IV: correlations between attentional variables and psychiatric diagnosis.

7.2. Additional explorative analyses. 206

8. DISCUSSION AND CONCLUSIONS. 219

SUMMARY. $\quad 235$

SAMENVATTING. 241

APPENDICES. $\quad 247$

REFERENCES. $\quad 281$ 
GLOSSARY OF VARIABLES IN THE INUESTIGATION

\section{DEPENDENT UARIABLE:}

(1) Psychiatric diagnosis according to DSM-III. Five categories, rank ordered according to degree of disturbance: normal, neurosis, schizotypal personality disorder, non-schizophrenic psychasis, schizophrenia.

\section{INDEPENDENT VARIABLES:}

Diagnostic variables:

(2) Affective Flattening. A 'schizophrenia variable', scored on a severity scale (Abrams and Taylor).

(3) Social Isolation. A 'schizophrenia variable', scored on a severity scale: the 'Social Functioning' section of the Schedule for Affective Disorders and Schizophrenia.

(4) Premorbid Asociality. A 'schizophrenia variable', scored on a severity scale: the Premorbid Asocial Adjustment Scale of Gittelman-Klein.

(5) Psychoticism (P). A self-rating scale, included in the Eysenck Personality Questionnaire (EPQ). A personality dimension postulated to be a measure of the predisposition to psychosis.

(6) Extraversion (E). A self-rating scale, included in the Eysenck Personality Questionnaire (EPQ), representing the introversionextraversion personality dimengion.

(7) Neuraticism ( $N$ ). A self-rating scale, included in the Eysenck Personality Questionnaire (EPQ), representing a personality dimension characterized by emotional instability.

(B) Lie (L). A self-rating scale, included in the Eysenck Personality Questionnaire (EPQ). It offers a measure of the tendency to 'fake good" * 
(9) SPEM Total (SPEM = Sinooth Pursuit Eye Movements). A measure (natural logarithm of 'noise' pover) of eye tracking performance during 35 seconds.

(10) SPEM Best Cycle (SPEM = Smooth Pursuit Eye Movements). Best score of eye tracking performance among the 14 period-scores. (The set of 14 periods of 2.5 seconds each constitutes the total eye tracking task lasting 35 seconds, scored in SPEN Total).

(11) Mean Reaction Time. The mean simple reaction time of regular and irregular series of trials with preparatory interval durations of 2,4 and 8 seconds.

(12) Reaction Time Redundancy Deficit. A ratio score: the mean reaction time of the regular series with a seconds preparatory interval durations divided by the mean reaction time of the trials with 8 seconds preparatory intervals included in the irregular series.

(13) CPT Simple Version Omission score (CPT = Continuous Performance Test). Percentage of amissions (false negative responses) during performance of the simple veraion of the CPT, a sustained attention task.

(14) CPT Complex Version Dmission score (CPT = Continuous Perfornance Test). Percentage of omissions (false negative responses) during performance of the complex version of the CPT, a sustained attention task.

(15) CPT Simple Version Commission score (CPT = Continuous Performance Test). Number of commissions (falae positive responses) during performance of the simple version of the CPT, a sustained attention task.

(16) CPT Complex Version Comission score (CPT = Continuous Performance Test). Number of commissions (false positive responses) during performance of the complex version of the CPT, a suvtained attention task.

Attentionel (electrophysiological) variables:

(17) CNV Cz Early Component (CNV = Contingent Negative Variation, the slow potential change of the EEG during warned reaction time performance). Amplitude of the early component of the CNV (preparatory interval 3.0 seconds), central derivation.

(18) CNV Cz Late Component (CNV = Contingent Negative Variation, the slow potential change of the EEG during warned reaction time performance). Amplitude of the late component of the CNV (preparatory interval 3.0 seconds), central derivation. 
(19) CNU Fz Early Component (CNV = Contingent Negative Variation, the slow potential change of the EEG during warned reaction time performance). Amplitude of the early component of the CNU (preparatory interval 3.0 seconds), frontal derivation.

(20) CNV Fz Late Component (CNV = Contingent Negative Variation, the slow potential change of the EEG during warned reaction time performanice). Amplitude of the late component of the CNV (preparatory interval 3.0 seconds), frontal derivation.

(21) PINV Cz (PINV = Post Imperative Negative Variation). Amplitude of the EEG after the imperative stimulus of the CNV paradigm, central derivation.

(22) PINV Fz (PINU = Post. Imperative Negative Variation). Amplitude of the EEG after the imperative stimulus of the CNV paradigm, frontal derivation. 

INTRODLCTION

Schizophrenia is a core concept of psychiatry since the early days of this science. Unfortunately, it has proven to be an ambiguous concept as well. It has nevertheless kept its place both in psychiatric research and in clinical practice. The least one can say, therefore, is that this concept fulfils a nasological need, and apparently serves as a specification of the more global concept of psychosis in general.

Theories of psychiatric disturbance, including schizophrenia, have stressed many different aspects, inside and outside the individual. The focus of interest has shifted many times, in accordance with prevaling paradigms and latest fashions. Most theories are one-sided and ignore the complexities of the human mind. Among the shortcomings of many theories are tendencies to reduce mental disturbance to isolated factors and to limit explanations to one single level of organization. The possibility of combinations and interactions of etiologically relevant characteristics is often ignored. Theoreticians also tend to mistake correlations for causal relations, and implicitely many theories suggest a dichotomy between geneticad and environmental influences. But most important, levels of analysis and description are confused. Many theories make errors of logical type.

Mental phenomena are of a different logical type than brain processes or than interpersonal communications. They transcend the boundaries of the brain and, consequently, of the individual. 'Consciousness does not exist in isolation' (Rose, 1976) - or, in another formulation: 
'The mental world - the mind - the world of information processing is not 1 imited by the skin" (Bateson, 1972). They certainly transcend analysis linited to one single level of description. Too often this fact is ignored, resulting in logical inconsistencies.

No attempt is made in this investigation to reduce the object of study - psychiatric disturbance, in particular the concept of schizophrenia to isolated deficits. But there is a central madel or framework of interpretation. This framework and its terminology are provided by the constructs of attention and information processing. The language of information processing is increasingly recognized as fruitful and rich in explaining capacity. It is currently a high ranking paradigm, in the Kuhnian sense (Kuhn, 1962), in the study of schizophrenia and related disorders. Attention, as an aspect of information processing, is the concept connecting the various performance and electrophysiological measures in this investigation. Its ambiguity, however, is acknowledged, as are the problems concerning the schizophrenia concept. These latter problems are considered to be the more serious ones. Attention is merely a unifying construction, and whether other labels or arrangements are considered more appropriate does not have to bother us here. This study is concerned with the relations between psychotic disorders and other psychiatric conditions, especially the concept of schizophrenia, and measures of individual dysfunctioning that can be subsumed under the head 'attentional dysfunctioning'.

Attentional mechanisms affer attractive possibilities to the study of psychiatric disturbance, because they are in a certain sense the bridge between individual and environment, although they primerily represent individual characteristics. Research studying the capacity and efficiency of attention and information processing offers promising conceptualizations of the contribution of individual cheracteristics to psychiatric disturbance. These developments are creating better perspectives to many research areas that threaten to stagnate by clinging to traditional diagnostic concepts. Traditional 
psychiatric explanations of disorganized behavior refer to states of minds in terms of symptoms and diseases. The problem is in describing unambiguously what these references imply. In research global descriptions of behavior, cognitions and emotions should be replaced by more precisely defined characterizations of psychiatric disturbance.

In addition attempts should be made to distinguish as far as possible between 'wired in' (genetically determined, endogeneous) features and acquired (learned, exogeneous) features although in practice it will be impossible to fully separate both aspects, because they are interwoven in complex genotype-environment interactions. Just like the acquired characteristics of the human mind, the genetically determined characteristics are derived from a broad range of largely continuously distributed posaibilities. Those individuals who fall into particular ranges of these distributions are at a higher risk for developing psychiatric illness, for instance schizophremia. They are more vullnerable to psychosacial and other kinds of stress. The potential for developing a (schizophrenic) psychosis is thus seen as deriving from the normal variability of trait distributions found within the population, in interaction with environmental influences. This means that schizophrenia is to be considered as comprising a set of disorders, and to be distinguished from other psychotic and non-psychotic psychiatric disorders only in an arbitrary way.

Individuals who find themselves in that particular range of the traits in question need not develop a schizophrenic psychosis. On the contrary, they may reach quite acceptable levels of adaptation, presumably depending on learning experiences and ather environmental influences. But it seems probable that many of them demonstrate their membership of a statistical minority in other unusual phenotypic characteristics, and often in minor degrees of paychiatrically" disordered behavior - the schizophrenia spectrum concept. 
When polythetic classifications of psychiatric disorders, based on the principles of numerical taxonomy, make progress in the near future, dysfunctions of attention and information processing are likely to rank high in the selected characters. The study presented here tries to provide indications about the relevance to these new developments of acme performance and electrophysiological measures of attention, by relating these measures to 'traditional' diagnostic classification, in particular the schizophrenia concept, and by comparison of these measures with some traditional schizophrenia characteristics scored dimensionally instesd of categorically, and with dimensional personality traits. 


\subsection{THE CONCEPT OF SCHIZOPHRENIA.}

\subsubsection{History.}

Psychiatric patients from various diagnostic categories are studied in this investigation. The diagnostic concept of schizophrenia, hovever, is in the centre of interest. Although the widespread use of the term schizophrenia since many years suggests soundness of the concept it denotes, clinical reality as well as research findings prove otherwise. Schizophrenia is what we define as schizophrenia. Therefore the history and the present use of this concept is briefly revieved here.

The history of the schizophrenia concept is dominated by the names of Emil Kraepelin and Eugen Bleuler. They had a strong influence upon the concept as we know it today, and after them relatively little has been added to the concept as such (Decker, 1978). Nevertheless, controversy exists until now about their central issues: types of symptoms considered typical and prognostic implications. As Wender (1963) has shown, the concept of a form of insanity, distinct from but often assaciated with affective alterations, characterized by a predilection for the young and a progressive course, had been identified halfway the ejghtteenth century already. Only the label was lacking until about 1860, when Morel used the name 'démence précoce'. 10 Years later 
Hecker descr" Ibed "Hebephreni " and Kahlbaum described "Katatonie'. Paranoia was a term already known for centuries and it is difficult to ascertain when it was first used in a sense app roximating its present interpretation. At the end of the nineteenth century Kraepelin (1896) published the fifth edition of his textbook. This edition included a subgroup of mental diseases based on age of onset and progressive deterioration. Under Morel's term dementia praecox he brought together Hecker's hebephrenia, Kahlbaum's catatonia and paranoia. In later editions dementia simplex was added. However, in these following editions, especially the eigth, he had to loosen the defining characteristics of age and prognosis and he was forced to rearrange the defining attributes in order to keep the entity.

The main point of Kraepelin's view was the correlation between symptomatolagy and prognosis. He distinguished dementia praecox from manic-depressive disorder, because he considered the prognosis of these conditions very different. He did not regard recovery as incompatible with the diagnosis of dementia praecox, but in his view paor prognosis and ultimate deterioration were very likely. The Kraepelinian conception of the disorder which Bleuler later renamed schizophrenia persists taday. 'True' schizophrenia is considered by many to be synonymous with paor prognosis schizophrenia. Kraepelin was primarily interested in symptoms. He did not try to understand the pattern of symptoms and the order in wich the symptomatology energes. Because of the strong influence he had on the current concept of schizophrenia, a summary of the symptoms he considered typical is mentioned (Kraepelin, 1904):

- Disturbance of attention.

- Hallucinations, most often acoustic, but also visual and tactile thallucinations.

- Thought and speech disturbances, varying from heightened distractibility to incoherence and neologisms.

- Delusions (of guilt, hypochondriacal, paranoid, grandiose). In particular delusions of control: the sensation of external control of behavior, thoughts and impulses. 
- Disturbances of affect. Inappropribte affect and especially blunted affect ("gemütiche Verblödung").

- Behavior disturbances and disordered motor functions: passivity, stupor, excitement, negativism, stereatypies, mannerisms, automatio obedience, catalepsia, echolalia and echopraxia.

About etiology Kraepelin was in doubt, of course, and he felt nothing solid could be said about it, but he believed the etiollogy could be somatic, probably metabolic. His construction of the concept was not primarily based om etiology, but on symptomatology and prognosis.

The contribution of Eugen Bleuler to the concept was essentially an addition and an attempt to bridge the gap between organic and psychodynamic interpretations of schizophrenia (Bleuler, 1911). He introduced the term schizaphrenia because, in mis opinion, dementia. was not essential and the term praecox incorrectly implied a delimitation to adolescence. He saw the schizophrenic syndrome as a splitting of the basic functions of the personality. In his opinion the prognosis of the schizophrenic disorders (he preferred to speak about a group of disorders instead of ane entity) was invariably poor, so in this respect he went further than Kraepelin: 'Mit dem Namen der Dementia Praecox oder der Schizophrenia bezeichen wir eine Psychosengruppe, die bald chranisch, bald in Schüben verläuft, in jedem stadium Halt machen oder zurückgehen kann, aber wohl. keine volle Restitutio ad integrim erlaubt. Sie wird charakterisiert durch eine spezifisch geartete, sonst nirgends vorkommende Alteration des Denkens und Fühlens und der Beziehungen zur Auszenwelt' (Bleuler, 1911).

Bleuler constructed a further arrangement of schizophrenic symptoma. He separated fundamental symptoms from accessory symptoms and primary symptams fram secundary symptoms. According to his view, primary symptoms are causied directly by etiologic agents, while the secundary symptoms represent the psychological reactions of the personality to the primary changes. Fundamental and primary symptoms are not necessarily the same. Fundamental symptoms are considered 
characteristic and present to an extent in every case of schizophrenia, whether latent or manifest. The accessory symptoms may ar ray not occur. Among the fundamental symptoms Bleuler included disorders of association, autism, ambivalence and blunted or inappropriate affect. Among the accessory symptoms he included the acute manifestations of the psychosis, like delusions, hallucinations and catatonic postures. Bleuler choose to establish the diagnosis on the basis of the clinical symptomatology in the initial stages of the disorder. In practice his concept of schizophrenia became wider than Kraepelin's dementia praecox. Etiology was a puzzle for Bleuler too. In his opinion nonorganic etiologic factors were unlikely however.

The concept founded by these two grandmasters in psychiatry has proved to be a strong one. Since then much research has taken place and much criticism has been uttered, but the concept of schizophrenia did remain vital and stimulating, although, no doubt, rather imperfect. Historically, two other men have been influential in preparing an increasingly broad definition of schizophrenia, especially in the United States: Adolf Meyer (1951) and Harry Stack Sullivan (1953). Meyer preferred to regard schizophrenia as a reaction type. Both stressed the importance of psychogenic factors in the environment, of distorted interpersonal relationships. This view on schizophrenia was further developed by investigators studying the interpersonal relationships in families of schizophrenic patients (e.g., Bateson, Jackson, Haley and Weakland, 1956; Wynne, Ryckoff, Day and Hirsch, 1958; Lidz, 1973). The contributions of Sigmund Freud in this area have also been influential. He was the first author who tried to axplain the content of psychoses in psychological terms. From his theory two main psychoanalytic orientations have developed, according to London (1973). The first one is the so-called unitary theory, viewing schizophrenia as being unconsciously determined in response ta intrapsychic conflicts, originating in infantile traumatic experiences. The second, specific, theory ascribes schizophrenia to a psychological deficiency state resulting from decathexis of mental representations of objects, drives, affects, and representations of 
time and space that have lost their immediacy. Among psychoanalytically oriented schools the tendency has been to diagmose schizophrenia primarily on the basis of the psychodynamic mechanisms, without considering the gravity of the symptoms or the course of the disorder. In this way the concept of schizophrenia became increasingly broad.

A line of continuity can be traced from these broadening conceptions to the attacks on the schizophrenia concept by Karl Menninger (Menninger, Mayman and Fruyser, 1963), who proposed to abandon all kinds of diagnostic categories, and by Thomas Szasz (1976), who even denied the existence of nonorganic mental illness, and above all strongly emphasized negative connotations and social implications of the diagnosis of schizophrenia. This view has been formalized in labeling theory, which sees the sacietal reaction as the main determinant of chronic mental illness, stabilizing rule breaking by labeling the person as deviant. In this particular case: as schizophrenic (Scheff, 1966; 1970).

\subsubsection{Etialogr and course.}

Of course there is more in the concept than the term. Neale and 0ltmanns (1980) rightly stated that the important question is not whether schizophrenia is an observable material entity, but rather whether it is a useful construct. Nevertheless we can readily agree that an etiologically based concept cannot be established today, at least not yet. It is generally accepted that both genetic and environmental factors aro involved in inextricable mutual interaction. It is hardly possible yet to specify these factors and in addition there is absolutely no reason to suppose that in aymptomatologically comparable cases the same etiological factors should have the same weights.

What is known about genetic factors in the etiology of solhizophrenia was recently clearly summarized by Gottesman (1978). Some of his 
tenets give an impression of the relevance of genetic factors.

- "The risk of schizophrenia to the relatives of index cases increases markedly with the degree of genetic relatedness (25\%, $50 \%$, 100\%) even in the absence of shared specific environments.

- The identical tuin concordance rates for schizophrenia are about three times those of fraternal twins and at least 30 times the general papulation rate.

- More than ane-half of the monozygotic pairs in recent studies are discordant for schizophrenia despite sharing all there genes in common; monozygotic and dizygotic twins as such are not at a higher risk for schizophrenia than singletons.

- Chyldren of normal parents crossfostered into homes where a parental figure became schizophrenic do not show an increased rate of schizophrenia.

- Identical twins reared apart from childhood are concordant for schizophrenia to about the same extent as those reared together'.

Whereas there were still problems with the early consanguinity studies, later tuin studies and especially adoption studies eliminated any doubts concerning the importance of genetic factors, although the specific nature of these factors still cannot be defined. Genetic research thus suggests that the contribution of the genes to the development of schizophrenia is considerable and may be as high as 50\%. It follows, on the other hand, that environmental factors play a substantial part in etiology too. Non-genetic somatic factors (e.g." pregnancy and bith complications) are likely to have some influence, but peychological and social factors are probably more important. The prevalence of schizophrenia is substantially higher in the lower social classes (Kohn, 1973; 1976). Correlates of social class probably play an etiological role, but secundary downward social mobility ('gocial drift') seems more important (Neale and 0ltmanns, 1980). Characteristics of the families of schizophrenics have received much interest. Special types of interpersonal relationghips within these families are supposed by many investigatars to predispose family members to s schizophrenic 
breakdown, and to perpetuate non-adaptive interactiona 1 patterns.

Major theories in this field are those of Bateson (Bateson, Jackson, Haley and Weakland, 1956), Wynne (Wynne, Ryckoff, Day and Hirsch, 1958) and Lidz (1973). Central to these theories is the assumption that schizophrenia is essentially learned inappropriate behavior. Much emphasis is placed on role theory and communication theory. The strongest evidence for links between family interaction patterns and schizophrenia has been praduced by investigatars studying disordered communication processes (reviewed in Goldstein and Rodnick, 1975 ; Jacab, 1975; Leff, 1978; Wynne, Toohey andi Doane, 1979; Liem, 1980). So far, predictions derived from these theories have not resulted in very convincing support. A major weakness is that all of these theories fail to explain why one family member falls ill and others remain well under similarly adverse conditions. In addition, the direction of the relationship between family interaction variables and schizophrenia, essential to the determination of the etiologic role of disordered family patterns, remains to be investigated.

Many parental attitudes and behaviors may be secondary reactions to disturbances primary originating in the child. Some experimental evidence exists that the tendency of schizophrenic patients to relapse is dependent on a high level of social stimulation, particularly emotions expressed by family members the patient is living with (Brown, Birley and Wing, 1972; Vaughn and Leff, 1976). These findings are in accordance with the supposedly protective effect of social isolation among schizophrenics. Identification of specific and essential experiences and transactional features may prove to be impossible, although overall the current literature suggeats that stressful life events may play some role in precipitating and in maintaining schizophrenic disturbance (discussion, for instance, in Neale and 0Itmanns, 1980). Perhaps quite nornal, routine experiences suffice to precipitate a schizophrenic psychosis in a vulnerable individual. The abnormality may lie entirely in the response of this individual to routine life events. On the ather hand, the theoretical 
subtlety of family interaction theories of schizophrenia mey have been neglected in mich research studying disordered interactional patterns in which schizophrenic subjects participated. Process and pattern, rather than etiology, are the focus of interest of many of these theories (De11, 1980).

Although there is no doubt that genetic factors play a role in predispasing to schizophrenia, this predisposition includes a spectrum of psychopathology and not merely schizophrenic psychoses (the so-called spectrum concept, to be discussed later on in this chapter). The exact nature of the predisposition is the subject of a large variety of hypatheses. Among these hypotheses are many that seek the basic defect in some biochemical abnormality. Examples of these types of hypotheses are the trans-methylation hypothesis, ascribing schizophrenia to toxic by-products of metabolism; hypotheses that postulate enzymatic deviancies, mainly focusing on elevated serum levels of creatine phosphokinase or on decreased levels of monoamine oxiadase activity in blood platelets; the endorphin hypothesis, which suggests that schizophrenia results from an excess of endogeneous opiate-like peptides; and the dopamine hypothesis, proposing an excessive function of dopaminergic pathways in the central nervous system. Recent reviews of research concerning these hypotheses are to be found in Meltzer and Stahl (1976); Carlsson (1978); Elliot and Berger (1978); Iversen (1978); Verebey, Volavka and Clouet (1978); Meltzer (1979); Bowers (1980); Wyatt, Potkin, Bridge, et al. (1980). These hypotheses offer suggestions about the pathophysiological characteristics of schizophrenia. This does not mean that they indicate the cause of this disorder. As stressed by Weiner (1980), the concept of a regulatory disturbance in systems in which several substances interact is intellectually more satisfying than a concept that accounts for this complex disorder on the basis of increases or decreases of one substance. In addition, altered biochemistry of the brain as well as deviant structural and regulatory characteristics are not: necessarily the result of genetically controlled variations. They may be as well under experiential control. 
A conception at another level of description was proposed by Meehl (1962). He suggested that the predisposition to schizophrenia is in a defect in neural integration, probably residing in the neuron's synaptic function. This view forms the basis of much of modern research on attention and information processing in schizophrenic patients.

The most attractive unifying construction, incorporating the seemingly conflicting approaches to the problem of etiology, is the vulnerability or diathesis-stress model. This model asserts that the varying schizophrenic psychoses are the result of a variety of predispositions to develop schizophrenia, in interaction with environmental stress. In the opinion of Gottesman and Shields (1973): 'The beauty of diathesis-stressor theory, or philosophy if you will, is that it fills the chasm between geneticism and environmentalism. Our preferred model for construing the syndrome of schizophrenia permits the clearer separation of aetiological and phenomenological considerations. It comprises a network of events connected by sequential causal arrows. A chain of consequences is set into motion by a variable polygenically caused predisposition and culminates in a set of symptams recognizable as schizophrenia. Feedback loops and chance have important roles in the total picture. Our construction clarifies how psychotherapy or phenothiazines or a good mother may each contribute to symptom amelioration without necessarily casting light on aetiological questions".

It is important to realize that, although hypotheses concerning schizophrenia stress many different aspects, ranging from phenomenology to biochemistry, these hypotheses are not necessarily mutually exclusive. Often they offer descriptions at different levels of analysis. In a certain sense psychosocial and interactional explanations are not less bjological than biochemical explanations. They simply refer to a far more complex level of biological organization. Likewise, there is only a seeming contradiction between hypotheses stressing predisposition and those stressing learning 
experiences. Mednick (1958), for instance, has proposed that schizophrenics suffer from genetically determined excessively strong autonamic and physiological reactions that habituate excessively slow In this model vicious circles create a generalization to, finally, an atypical anxiety-reducing avoidant behavior. Schizophrenia has to be taken as the result of complex interactions between individual and environment. Certain patterns of parent-child interaction may lead to imperfections in personality (ego) development, leading in turn to progressive failures in social and relational adaptave capabilities when the child grows older. Why the failure of adaptation takes the particular form of schizophrenic symptoms and behavior is largely unknown. The explanations will have to be sought both in individual functional characteristics, to a considerable degree genetically determined, and in social-interactional patterns.

Since Kraepelin described ('invented", Szasz (1976) would say) dementia praecax a continuing question has been whether this disorder does involve a single process, a group of related processes, or is actually a number of different disorders. This question is reflected in efforts to solve the problem of etiology by identifying subtypes. There are many approaches to subtyping schizophrenia (e.g., the classic paranoid, hebephrenic, catatonic and simple types; acute us. chronic; process vs. reactive; paranoid vs. nonparanoid; good vs. poor premorbid), but until now they have not helped us very much in clearing problems of etiology. We should accept that there may be many kinds of etiology, and that the manifest form of the schizophrenic syndrome can be arrived at by different pathways. This phenomenon-behavioral end products appearing similar, but being subserved by different processes - is well known in biology and is called functional convergence.

Likewise, there are certainly also great variations in the outcome of schizophrenic psychotic episodes as to social, interpersonal and symptom-free functioning. A specific course of schizophrenia does not exist. The large scale transcultural follow-up study of the World 
Health Organization, the International Pilot Study of Schizophrenia, has demonstrated great variability of outcome after 2 years, with better outcome in underdeveloped countries than in developed countries (WHO, 1979). In recent years several long-term follow-up studies of schizaphrenia, confined to a single culture, have been published too. Bleuller (1972; 1978) did a careful follow-up investigation after 22 years of 208 schizophrenics. Ciompi and Muller (1976, 1980) re-examined 289 schizophrenics after an average duration of 36,9 years. Finally, Gross, Huber and Schenttler (1979; 1980) did follow-up of 502 schizophrenics after an average of 21,4 years. The general concordance of these studies is striking. Favourable psychiatric conditions at re-examination were found in 53 per cent of cases by Bleuler, 49 per cent of cases by Ciompi and Muller and 57 per cant of cases by Huber and co-workers. Of course the course and outcome of a schizophrenic psychosis may be influenced by many external factors, e.g., treatment facilities, social class, social support, but the relevance of these influences is difficult to ascertain.

\subsubsection{Diagnostic criteria.}

Although the tradition persists today that a definite diagnosis of schizophrenia should be made only if symptoms persist chronically and if a deteriorating, unremitting course is followed, agreement has been widespread in recent years that there is no alternative to using symptomatology as the basis for the definition of schizophrenia. If we define a schizophrenic psychosis as a condition with a particular outcome (or treatment response) diagnosis has to be postponed for months, possibly for years. But some of the most important functions of diagnosis are to determine treatment and to predict outcome: Because these implications determine to a large degree the usefulness and validity of a classification, it seems important to place boundaries in such a way that categories are as homogeneous as possible with respect to prognosis. The problem of the traditional vay of assigning diagnosis to psychiatric patients is that it is based on knowledge of typical symptoms and is learned to a large degree by 
modelling processes. The result is that diagnostic concepts as they are clinically used are not securely anchored, and continuousily change, at different speeds and to differing extents in different countries and centres.

International differences in diagnostic criteria have been studied, among others, by the so-called US/UK Diagnostic Project, investigating the differences between New York and London (Cooper, Kende11, Gurland, et a1., 1972). The American schizophrenia concept appeared to embrace substantial parts of the British concepts of depression, neurosis, personelity disorder and most of the concept of mania. Within the United States substantial differences thave been recorded between different centres and not everywhere the concept appeared to be used as broad as by the New York psychilatrists, participating in the US/UK Diagnostic Project (Sharpe, Gurland, Fleiss, et al., 1974). In Eurape Kende11, Pichat and von Cranach (1974) compared diagnoses made from diagnostic interviews by psychiatrists from three countries: United Kingdom, France and Germany. They found no significant differences in the global concepts of schizophrenia used by these three groups of psychiatrists. The best information about schizophrenia criteria in many other parts of the world, including developed as well as developing countries, has been provided by the already mentioned International Pilot Study of Schizophrenia (WH0, 1973). Differences were not great between most of the participating centres, including centres in the United Kingdom, Czechoslovakia, Denmark, Nigeria, India, Colombia and Taiwan. The centres in the United States and the USSR, however, appeared to use considerably broader concepts.

The apparent need for an international classification of mental diseases has been recognized since many years. The World Health Organization has developed classifications of diseases since it came into being in 1948 (the International Statistical classification of Diseases, Injuries and Causes of Death-ICD). By now the ninth revision of this international classification is in use. Although it is still far from ideal with respect to the classification of psychiatric 
disorders, including schizophrenia, progress has continuously been made across the successive editions of this classification (discussion in Kendell, 1975).

It is difficult to evaluate the extent to which these classifications have influenced the clinical use of diagnostic concepts like schizophrenia. It does not seem to be of much significance so far for the use of diagnosis in research investigations. The reason seems that it is difficult and perhaps impossible to reconcile the process of reaching international consensus with the aims and interests of investigators. Therefore, independent of these international efforts, research classifications have been developed and more especially, in line with our interests here, a number of research criteria for the diagnasis of schizophrenia have come into more or less widespread use.

Current research definitions of the schizophrenia concept are mainly based on symptoms. Which symptoms or combinations of symptoms should form the basis of the definition is a matter of controversy however. The tradition that gives primacy to symptomatology rather than course of dysfunctioning starts, perhaps, with Eugen Bleuler and his hierarchy of primary and secundary symptoms. In recent years several. primarily symptom based schizophrenia definitions have been developed that are in addition more or less operationalized. This means, they contain rules of application, they stipulate which symptoms or combinations of symptoms are adequate to establish the diagnosis. They are a considerable improvement on those former conceptions that only described the typical clinical features of schizophrenia, but did not indicate the necessary conditions for a diagnosis of schizophrenia. An example of such a vague conception was the definition of achizophrenia by the second Diagnostic and Statistical Manual (DSM-II) of the American Psychiatric Association (1968): 'This large category includes a group of disorders manifested by characteristic disturbances of thinking, mood and behaviar. Disturbances in thinking are marked by alterations of concept formation which may lead to misinterpretation of reality and sometimes to delusions and hallucinations, which 
frequently appear psychologically self-protective. Corollary mood changes include ambivalent, constricted and inappropriate emotional responsiveness and loss of empathy with others. Behavior may be withdrawn, regressive and bizarre".

But the diversity in operational definitions creates new problems. Which definition has to be preferred? Independent validating criteria for a diagnosis of schizophrenia are lacking. Prognosis is considered by many to be the only variable acceptables as a validating criterion, but autcome is not a simple or unitary function (Schwartz, Myers and Astrachan, 1975; Strauss and Carpenter, 1978). The results of the International. Pilot Study of Schizophrenia (WHO, 1979) further reduce the attractiveness to organize diagnostic systems according to this dimension. Of course, other sources of validating evidence may be found, for instance in drug response and in biological and performance marker"s. In the near future progress is likely to be made in this area of validating criteria. It seems likely, however, that numerical taxonomic methoda, to be discussed later on in this chapter, will eventually prove to be the best way of classification in psychiatry, at least for research purposee.

To illustrate the diversity in schizophrenia criteria currently in use for research purposes, the most important and influential of these diagnastic systems are mentioned now. The differences between these Bystems and the conflicting evidence concerning predictive validity will be briefly discussed afterwards.

a. Kurt Schneider"s first rank symptoms (Schneider, 1962).

1. Audible thoughts.

2. Hearing two or more voices discussing the patient or arguing about him.

3. Hearing voices commenting on one's actions.

4. The experience of being a passive and reluctant recipient of bodily sensations imposed by some external agency.

5. Thought withdrawal.

6. Thought insertion.

7. Thought broadcasting. 
B. delusional perception.

9. The experience of reelings as if under external control.

10. The experience of impulses that are felt not to be one's oun.

11. The experience of movements and acts as initiated and directed by a controlling influence.

Schneider did not consider these symptoms as primary symptoms in the Bleulerian sense, but as symptoms with a special diagnostic significance. In his opinion they are sufficient but not necessary to establish the diagnosis of schizophrenia. The presence of one symptom suffices, in the absence of arganic brain disease or other somatic pathology of possible etiological significance.

b. Langfeldt"s criteria (Langfeldt, 1960).

1. A special type of emotional blunting followed by lack of initiative and altered frequently peculiar behavior.

2. Catatonia. Periods of restlessness and stupor with the characteristic signs of negativism, catalepsy and vegetative symptoms.

3. Paranoid symptoms with depersonalization and derealization, experiencing the disturbance as originating outside himself.

4. Paranoid symptoms with primary delusions.

5. Chronic hallucinations not due ta organic disease.

Just as Schneider proclaimed of his criteria, each of these symptoms, according to Langfeldt, suffices for a diagnosis of schizophrenia. These criteria are considered representative of the rather conservative Scandinavian view on schizophrenia.

c. New Haven Schizophrenia Index (Astrachan, Harrow, Adler, et al., 1972).

1. (a) Delusions (other than depressive).

(b) Hallucinations (auditory).

(c) Hallucinations (visual).

(d) Hallucinations (other).

2. (a) Bizarre thinking.

(b) Autism or grossly unrealistic private thoughts.

(c) Looseness of association, illogical thinking, overinclusion.

(d) Blocking. 
(e) Concreteness.

(f) Derealization.

(g) Depersonalization.

3. Inappropriate affect.

4. Confusion.

5. Paranoid ideation (self-referential thinking, suspiciousness).

6. Catatonic behavior.

(a) Excitement.

(b) Stupor.

(c) Waxy flexibility.

(d) Negativism.

(e) Mutism.

(f) Echolalia.

(g) Stereotyped motor activity.

To be considered schizophrenic a patient must score on item 1 or 2 (a), 2 (b) , 2 (c) and must attain a total score of at least 4 points: 2 points for items 1 (a) - (d) and 2 (a) - (c) but no more than a total of 2 for hallucinations, 1 point for $2(d)-(g)$ and $3,4,5,6$. When the 4th point necessary for inclusion in the schizophrenic group is provided by 2 (d) or 2 (e), these symptoms are not scored.

This diagnostic system offers a broad concept of schizophrenia. It formalizes a clinical stereotype of schizophrenia primarily based on thought disorder and on delusions and hallucinations. The authors present evidence that this checklist provides a good reflection of the broad American approach to diagnosing schizophrenia.

d. St. Louis Criteria (Feighner, Robins, Guze, et al., 1972).

A through $C$ are required.

A. Both of the following are necessary:

(1) A chronic illness with at least six months of symptoms prior to the index evaluation without return to the premorbid level of psychosocial adjustment.

(2) Absence of a period of depressive or manic symptoms sufficient to qualify for affective disorder or probable affective disorder.

B. The patient must have at least one of the following:

(1) Delusions or hallucinations without significant perplexity or disorientation associated with them.

(2) Verbal production that makes communication difficult because of a lack of logical or understandable organization. (In the presence of muteness the diagnostic decision must be deferred).

c. At least three of the following manifestations must be present 
for diagnosis of "definite" schizophrenia, and two for a diagnosis of 'probable' schizophrenia.

(1) Single.

(2) Poor premorbid social adjustment or work history.

(3) Family history of schizophrenia.

(4) Absence of alcoholism or drug abuse within one year of onset of psychosis.

(5) Onset of illness prior to age 40.

This system requires more than the mere presence of certain symptoms. The requirement that the illness must have been continuously present for six months before a diagnosis can be made, implicates that during this period diagnosis has to be postponed.

e. Flexible System for a diagnosis of schizophrenia (Carpenter, Strauss and Bartko, 1973).

(-) Indicates that absence of the criterion favors a diagnosis of schizophrenia.

(1) Restricted affect.

(2) Poor insight.

(3) Thoughts aloud.

(4) Waking early (-).

(5) Poor rapport.

(6) Depressed facies $(-)$.

(7) Elation (-).

(B) Widespread delusions.

(9) Incoherent speech.

(10) Unreliable information.

(11) Bizarre delusions.

(12) Nihiliatic delusions.

Depending on the number of symptoms a patient has, the diagnosis of schizophrenia becomes increasingly certain, in terms of likelihood that investigators from diverse centers all over the world would also consider that patient schizophrenic. Thresholds advocated by the authors are 5 of 6 symptoms. The diagnostic system has been developed from data from the International Pilot Study of Schizophrenia (WHO, 1973).

f. Catego (Wing, Cooper and Sartorius, 1974).

This is a computer program, incorporating diagnostic rules and 
based on the items of the Present State Examination, a structured interview.

Class St.

This class contains the central schizophrenic conditions. The characteristic symptions are:

(1) Thought intrusion, broadcast or withdrawal.

(2) Delusions of control.

(3) Voices discussing the patient in third person or commenting on thoughts or actions.

(4) Dther auditory hallucinations (not affectively based).

(5) Dther delusions.

If any of the first 3 symptoms is present, the patient is automatically allocated to class $5+$, similarly if both symptoms (4) and (5) are present. (Other psychotic classes, related to the schizophrenie concept, are P+ (paranoid psychases), O+ (other peychoses), 0 ? and $P$ ?

9. Research Diagnostic Criteria for Schizophrenia (Spitzer, Endicott and Robins, 1978 (a) and (b)).

A through $\mathbb{C}$ are required for the period of illness being considered.

A. During an active phase of the illness (may or may not now be present) at least two of the following are required for definite and one for probable:

(1) Thought broadcasting, insertion, or withdrawal.

(2) Delusions of being controlled (or influenced), other bizarre delusions, or multiple delusions.

(3) Somatic, grandiose, religious, nihilistic, or other delusions without persecutory or jealous content lasting at least one week.

(4) Delusions of any type if accompanied by hallucinations of any type for at least one week.

(5) Auditory hallucinations in which either a voice keeps up a running commentary on the subject's behavior or thoughts as they occur, or two or more voices converse with each other.

(6) Non-affective verbal hallucinations spoken to the subject.

(7) Hallucinations of any type throughout the day for several days or intermittently for at least one month.

(8) Definite instances of marked formal thought disorder accompanied by either blunted or inappropriate affect, 
delusions or hallucinations of any type, or grossly disorganized behavior.

B. Signs of the illness have lasted at least two weeks from the onset of a noticeable change in the subject's usual condition (current signs of the illness may not now meet criterion $A$ and may be residual symptoms only, such as extreme social withdrawal, blunted or inappropriate affect, mild formal thought disorder, or unusual thoughts or perceptual experiences).

C. At no time during the active period (delusions, hallucinations, marked formal thought disorder, bizarre behavior, etc.) of the illness being considered did the subject meet the full criteria for either probable or definite manic or depressive syndrome to such a degree that it was a prominent part of the illness.

These schizophrenia criteria were specifically developed to enable research investigators to obtain relatively homogeneous groups of subjects. The authors assumed that for research purposes it is better to avoid false positives than false negatives. Therefore the Research Diagnostic Criteria exclude patients with significant affective symptoms from the schizaphrenic group.

Many of the terms and criteria used in the RDC are part of the revised nomenclature of the American Psychiatric Association DSM-III (APA, 1980). The DSM-III criteria for a diagnosis of schizophrenia (see appendix-1) represent the most recently developed demarcation of the schizophrenia concept. They are discussed in Chapter 5.1.1., because they were preferred for diagnostic classification in the present investigation.

The foregoing review of schizophrenia criteria is illustrative of the confusion concerning the boundaries of the schizophrenia concept. Mutual differences between these diagnostic systems are great. The criteria differ in the proportion of patients diagnosed as schizophrenic and they disagree about which particular patients qualify for the diagnosis. The more restrictive criteria do not merely capture the central core of the concept as it is clinically 
used, but the diagnastic systems represent essentially different concepts of chizophrenia, as is shown by several studies comparing these diagnostic systems.

In a study by Brockington, Kendell and Leff (1978) the numbers of patients classified as schizophrenic according to the definitions discussed here ranged from 10 to 45 (out of 119) and, in arother selection of patients, from 24 to 56 (out of 134). Using nearly the same sete of criteria, Strauss and Gift (1977) diagnosed from 4 to 68 (aut of 272) patients as schizophrenic. Overall and Hollister (1979) cancluded that of 166 clinically diagnosed schizophrenics the Flexible System (at the level of 5 symptoms) accepted 136, the Research Diagnostic Criteria (probable plus definite) 122 and the 5 t. Louis Criteria only 44 as schizophrenic. Their investigation of the percentages of disagreement gave the following results: Percentages were for Flexible System versus Research Diagnostic Criteria 31.4 , for Flexible System versus St. Louis Criteria 59.0 and for Research Diagnostic Criteria versus St. Louis Criteria 50.6. These findings illustrate the condition that the different diagnostic systems represent different concepts of schizophrenia. Taken together, these studies suggeat that the New Haven Schizophrenia Index, the Flexible System ( 5 or more symptoms) and Catego (broad interpretation) are braad conceptions. Narrow conceptions are the St. Louis Criteria and the Research Diagnostic Criteria (definite). Research Diagnostic Criteria (probable) seems intermediate, although fairly broad.

Differences between the various diagnostic systems, including DSM-III, at the level of signs and symptoms can be illustrated as follows.

The Flexible System does not permit a diagnosis of schizophrenia on the basis of hallucinations, but the Research Diagnostic Criteria qualify for schizophrenia when hallucinations of any type are present for several days or intermittently for one month, and DSM-III when hallucinations on several occasion are auditory, not limited to a few words and not related to mood disturbance, or when they occur in 
combination with formal thought disorder.

The First Rank Symptoms require special types of delusions, notably thought withdrawal, insertion or broadcasting, or feeling, impulses or movements under external control, and Langfeldt's Criteria require 'paranoid symptoms" or primary delusions. On the other hand, the Research Diagnostic Criteria are content with delusions of any type, if accompanied by hallucinations for one week, and in DSM-III this required time period has even disappeared. Special types of delusions, related to those of the First Rank Symptoms, suffice for a diagnosis of schizophrenia in these last two systems.

Formal thought disorder does not form part of the First Rank Symptoms, Langfeldt's Criteria and Catego, and consequently a diagnosis of schizophrenia cannot be made on this basis according to these diagnostic systems. The New Haven Schizophrenia Index, however, contains a detailed section on formal thought disorder, and a diagnosis of schizophrenia can be based here purely on this symptom.

The First Rank Symptoms, the St. Louis Criteria, the Flexible System and Catego do not allow a diagnosis of schizophrenia on the basis of disordered motor behavior (catatonia), but according to Langfeldt's Criteria this symptom suffices for this diagnosis.

Blunted, flat or inappropriate affect is not included in the First Rank Symptoms, the St. Louis Criteria or Catego, but, like the afore mentioned symptom, it suffices for a diagnosis of schizophrenia according to Langfeldt's Criteria.

Differences are also manifest in the added requirements. For the First Rank Symptoms and Catego, the mere presence of certain types of symptoms suffices. The St. Louis Criteria, however, put additional requiremente not related to symptomatology, and a duration of six months of symptoms is required, like by DSM-III (although prodromal or residual phases are allowed here). 
Finally, the New Haven Schizophrenia Index excludes depressive delusions and in the flexible System some indicators of mood disturbance favor a diagnosis of schizophrenia if absent. The St. Louis Criteria and the Research Diagnostic Criteria require absence of a fully developed depressive or manic syndrome, while DSM-III permits these syndromes anly when developing after any psychotic symptom, or when brief in duration. The other diagnostic systems do not pay attention to depressive or manic symptoms.

It may be clear from this overview that the differences between the various diagnostic systems are great and that these differences concern all types of symptoms considered typical for a diagnosis of schizophrenia by one or more diagnostic systems. The consequences for research making use of any of these diagnostic systems are important. While reliability may increase, comparability between studies using different diagnostic systems remains a problem.

Comparing the sets of schizophrenia criteria reviewed here with regard to their predictive validity, conclusions are also diverging. In any case, the New Haven Schizophrenia Index and the First Rank Symptoms are consistently considered poor prognostic indicators (Brockington, Kendell and Leff, 1978; Bland and 0rn, 1979; 1980; Knight, Roff, Barnett and Moss, 1979). Conclusions differ about the prediotive validity of the St. Louis Criteria (Bland and Orn, 1979; Johnstone, Firth, Gold and Stevens, 1979; Tsuang, Woolson and Fleming, 1979), Langfeldt's Criteria (Strauss and Carpenter, 1974; Hawk, Carpenter and Strausa, 1975; Brockington, Kendell and Leff, 1978) and the Flexible System (Hawk, Carpenter and Strauss, 1975; Brockington, Kendell and Leff, 1978). Predictive validity of the narrov version of Catego has appeared poor in the International Pilot Study of Schizophrenia (WHO, 1979), but the broadest version was judged more positively by Brockington, Kendell and Leff (1978). Predictive validity of the Research Diagnostic Criteria was judged good by the latter authors, but only for the broad form ("probable" schizophrenia suffices). A recent comparison study favored the $5 t$. Louis and DSM-III 
criteria concerning predictive validity, while the Research Diagnostic Criteria and Catego showed less specificity in this respect (Helzer, Brockington and Kendel1, 1981). Because of the conflicting evidence on their predictive validity, it is rather difficult at the moment to make a choice among the various diagnostic systems on this basis alone. What about the predictive validity of symptoms taken individually instead of in the form of diagnostic systems?

The currently most reliable answers to this question have come from the 2-year follow-up of the International Pilot Study of Schizophrenia (IPSS) (WHO, 1979). One of the major findings of this transcultural psychiatric investigation of 1200 patients in 9 countries is the great variability of course and outcome of patiente who correspond rather closely to the concept of schizophrenia inherent in the Catego program. One third of those followed up were totally asymptomatic at second year follow-up. As a group, however, the schizophrenic patients did more poorly over the 2-year follow-up period than patients with other functional psychoses. Although the range of variability in the course and outcome of schizophrenia has not generally been assumed to be as broad as suggested by this follow-up study, the concepts of good and poor outcome of schizophrenia have been present in the literature for a long time, and many attempts have been made to identify various symptomatic, past history and sociodemographic factors that are highly correlated with good or poor outcome. Results of the 2-year follow-up of the IPSS are, that no single predictor variable was capable of explaining a large proportion of the variance of any of the course and outcome measures of the schizophrenic patients. Optimal combinations of the five predictors with the highest predictive power could only explain from 8 to 22 per cent of the variance of any of the course and outcome variables. The three clusters of predictors: sociodemographic variables, past history variables and characteristics of the episode of inclusion (including initial symptomatology), appeared about equal with regard to their predictive power. 
The best predictors within the class of sociodemographic variables were social isolation (associated with poor outcome) and marital status (uidowed, divorced or separated associated with poor outcome, married associated with good outcome). The best past history predictors were history of past psychiatric treatment, poor psychosexual adjustment, and unfavourable environment, all associated with a poor outcome. The best predictors among the characteristics of the episode of inclusion were long duration of the episode of inclusion prior to initial evaluation and insidious onset (both associated with poor outcomey.

Poor premorbid social adjustment has long been recognized as an indicator of the poor prognosis or process type of schizophrenic psychosis. A recent report from a follow-back study (Lewine, Watt, Prentky and Fryer, 1980) confirmed the relevance of poor childhood social competence (of which, of course, psychosexual adjustment forms a major part) to the concept of schizophrenia. The results indicated that children eventually hospitalized for schizophrenia (as clinically diagnosed) are characterized by poor interpersonal skills long before symptom onset. Scores for interpersonal competence (based on teachers" records) were significantly lower for schizophrenics than for psychotic depressives, neurotics and normals. Only the difference with personality disordered patients was not significant.

In view of the fact that symptoms are to a large degree the defining characteristics of the schizophrenia concept as it is currently used, especially by research investigators, the predictive capacity of psychiatric symptoms is an interesting research area. It is important to note, therefore, that individually symptoms did not emerge as very good predictors in the IPSS. Some predictive power was associated with derealization, affective symptoms, psychophysiological symptons, circumstances related cooperation difficulties (associated with good outcome) and flatness of affect and indication of personality change (assaciated with bad outcome). The predictive values of a total of 129 symptoms as assessed on initial evaluation were investigated by 
means of multivariate statistical techniques, with messures of pattern of course and overall outcome as dependent variables. 67.8 Per cent of the variance in overall outcome could be explained by the 129 selected symptoms, only data on patients falling within the extreme and contrasting categories of very favourable outcome versus very unfavourable outcome being used in the analysis. Selection of optimal predictive combinations of symptoms by stepwise multiple regression analysis gave the results presented in Table 1.

The conclusion has to be that whenever a set of schizophrenia criteria has predictive validity, this can only to a limited extent be the result of chaice of symptoms. It must be remembered however, that as a group the schizophrenic patients in the IPSS had a worse course and outcome than patients with affective psychoses, although course and outcome of schizophrenia varied in different centres, and were less severe in developing countries than in developed countries. In developed countries (Czechoslovakia, Demmark, USSR, UK, USA) differences between these two diagnostic groups were statistically significant for every course and outcome variable, but in developing countries (Colombia, India, Nigeria, Taivan) these differences, although in the same direction, did not reach levels of statistical significance for any of the course and outcome variables. As symptoms were the defining characteristics of the patient groups, the fact that, at least in the developed countries, the schizophrenic group had a worse course and outcome than patients with affective psychoses, indicates that the symptoms distinguishing the two groups of patients do have predictive power in these countries.

Two years is a rather short period of time, but a five-year follow-up study of the American IPSS cohort of schizophrenic patients stili concluded that, despite overlap, the mean outcome in the schizophrenic cohort was poorer than in the non-schizophrenic patients. At five-year follow-up restricted affect was the only symptom with predictive power (poor outcome) (Hawk, Carpenter and Strauss, 1975). 
Iable 1.

20 SYMPTOMS (UNITS OF ANALYSIS) EMERGING AS BEST PREDICTORS OF OVERALL OUTCOME CATEGORIES I OR 5 IN SCHIZOPHRENIC PATIENTS. (RESULTS OF STEPWISE MULTIPLE REGRESSION ANALYSIS).

\begin{tabular}{|c|c|c|c|}
\hline $\begin{array}{l}\text { Multiple } \\
\text { Regression } \\
\text { Step No. }\end{array}$ & Units of Analysis & $\mathbb{R}^{2}$ & $\begin{array}{l}\text { Dutcome } 1(+) \\
\text { Dutcome } 5(-)\end{array}$ \\
\hline 1 & Early waking & .073 & + \\
\hline 2 & Suicidal thoughts & .105 & - \\
\hline 3 & Overactivity & .133 & + \\
\hline 4 & Flatness of affect & .155 & - \\
\hline 5 & Warries & .183 & - \\
\hline 6 & Change in sex behavior & .206 & - \\
\hline 7 & Ambivalence & .227 & + \\
\hline 8 & Mutism & .244 & - \\
\hline 9 & Negativism & .267 & + \\
\hline 10 & Derealization & .287 & + \\
\hline 11 & Repetitive movements & .307 & - \\
\hline 12 & Fantastic delusions & .324 & - \\
\hline 13 & Speech dissociation & .337 & - \\
\hline 14 & Situation anxiety & .349 & - \\
\hline 15 & Speech impediments & .363 & + \\
\hline 16 & Mannerisms & .374 & + \\
\hline 17 & Lability of affect & .383 & + \\
\hline 18 & Haughtiness & .398 & - \\
\hline 19 & Delusions of persecution & .408 & + \\
\hline 20 & Social withdrawal & .417 & - \\
\hline
\end{tabular}

$R^{2}$ for 129 unite of analysis $=0.8232$, corresponding to 67.8 per cent explained variance.

(+) predicts overall outcome category 1 (very favourable outcome).

(-) predicts averall outcome category 5 (very unfavourable outcome). From: World Health Organization (1979). 
Pope and Lipinski (1978), who reviewed the relevant literature, also concluded that schizophrenic symptoms have virtually no demonstrated walue in predicting outcome in psychoses, but that affective symptoms do appear to have demonstrable value. Their principal conclusion was, that no knoun pathognomonic symptoms for schizophrenia, nor even any clusters of symptoms, taken in cross section, have yet adequately been demonstrated to be walid in diagnosing schizophremia. But conclusions of this type seem to be the product of circular reasoning. The unfavourable prognostic significance of emational blunting or affective flattening (which was excluded by Pope and Lipinsky from their definition of schizophrenic symptoms) was also stressed by Astrup and Nareik (1966) in their long-term follow-up study. A restrospective study by Knight, Roff, Barrnett and Moss (1979) confirmed the association of affective deficit (and also of poor social adjustment) with poor outcome after 22 years. In fact, emotional blunting or flat or inappropriate affectivity has been considered a core symptom of schizophrenia by many psychiatrists since the syndrome was originally described. There may be some differences among investigators in their use of these terms, but as these differences are seldom made explicit they have to be neglicted here. Although this symptom has been regarded a marker of poor outcome more than a predictor per se, both the Hawk et al. (1975) study and the knight et al. (1979) study have made it unlikely that affective deficit is merely the result of chronicity itself. Instead, it seems to be a feature that may be present during every stage of the disorder.

To some degree attempts to delimit the concept of schizophrenia by stressing aspects with prognostic implications have their counterpart in the spectrum concept of schizophrenia. The spectrum concept maintains that there exists a spectrum of psychiatric states and behavior deviancies, some psychotic and others not, which share a genetic etiology with schizophrenia (Rosenthal, 1974, 1975, 1978; Reich, 1976; Haier, Rosenthal and Wender, 1978). Any particular schizophrenia spectrum disorder is seen as a point on a continuum, 
with differences among the points reflecting differences in intensity, or in some other clinically evident quality. These differences are genetically and environmentally determined. Controversy exist about which types of disorders should be considered as belonging to the schizophrenia spectrum and which not. Cottesman and Shields (1976), for instance, concluded in a critical review, that it is doubtful whether schizoid personality as assessed at that time belongs to the spectrum. Where to place the acute, the remitting and the schizoaffective psychoses they also considered still a problem. According to these authors, some of these types of psychoses may fall outside the spectrum, others may be part of it.

Ever since Bleuler (1911) described latent schizophrenia to classify nonpsychotic individuals with underlying forms of psychopathology considered similar to those of patients diagnosed as schizophrenic, the boundaries of the schizophrenia concept have received interest. Terms like borderline schizophrenia, pseudoneurotic schizophrenia and borderline personality organization seemingly have a cansiderable averlap, but there are important differences too. Often these terms seem to be used rather indiscriminately. Reliability and validity of the borderline concept are still a problem, and the delimitation of the borderline concept from schizophrenia as well as from the affective disorders is a matter of controversy. Controversy is most evident, hovever, in the narrover question of whether separate borderline conditions should be distinguished, for instance, according to their relationship primarily with affective disorders or primarily with schizophrenia (Liebowitz, 1979). Perry and Klerman (1978) compared four sets of criteria for the diagnosis of borderline syndrome, namely those of Knight (1953), Kernberg (1967), Grinker, Werble and Drye (1968) and Gunderson and Singer (1975). From a total of 104 criteria encompassing mental status, history, interpersonal relationships, defense mechanisms, and other judgements of personality functioning, half appeared to be mentioned in only one of the four diagnostic descriptions. This striking lack of agreement characterizes the current situation. It may be that differentation 
between subtypes will bring clarity in this issue. In any case, Spitzer et al. (Spitzer, Endicott and Gibbon, 1979; Spitzer and Endicott, 1979) have defended the separation of a condition they labeled Schizotypal Personality Disorder from Borderline Parsonality Disorder, because of the relative independence of the behavioral characteristics of these two conditions. They have provided evidence that the borderline concept is not unitary and is best conceptualized as consisting of at least two major dimensions that are relatively independent within a borderline group. It is the concept of schizotypal personality disorder which is important here, because of its special relationship with the schizophrenia concept. This condition, as included in DSM-III, is defined by a set of 8 items (for details: see appendix-1).

The schizatypal items were derived by Spitzer at al, by reviewing the case records of patients diagnosed by Kety, Wender, Rosenthal et al. (Kety, Rosentha1, Wender and Schulsinger, 1968; 1971; Rosenthal, Wender, Kety et al. 1971) as being within the schizophrenia spectrum as defined by these investigators. The schizotypal features therefore have a purely descriptive function. They signify persons who have clinical features that resemble the featurea of patients with prodromal or residual schizophrenia (Spitzer and Endicott, 1979). (DSM-III creates the diagnosis of schizotypal personality disorder in addition to the diagnosis of schizoid personality disorder. The latter category lacks the eccentricities of communication, behavior, or thought characteristic of the schizotypal category). According to Siever and Gunderson (1979), who reviewed the relevant literature, firm conclusions about the genetic determinants of borderline conditions in general are not allowed. The evidence for a genetic relationship between schizotypal features and achizophrenia, however, although inconclusive at the moment, was considered suggestive by these authors. Recent support for this contention was offered by Kendler, Gruenberg and Strauss (1981). 
The term 'achizotypic' was originally used by Meehl (1962) in his theory of achizophrenia. Aecording to this theory some persons he labeled 'schizotypics" present characteristics he considered indications of a neural integrative defect, which he labeled "3chizotaxia". All schizataxic persons become schizotypic in personality organization, but only a minority becomes clinically schizophrenic, a process that is dependent on certain environmental conditions. So schizotaxia is considered a necessary but not sufficient condition in the etiology of schizophrenia. Although there are important differences between the schizotypic features of Meehl and those of DSM-III, the basic hypotheais has maintained its position in current theorizing on the schizophrenia concept, schizotaxia being relabeled as vulnerability.

In discussing both the search for features characteristic of the 'core' schizophrenic ayndrome and the search for features of borderline conditions the fundamentel dilemma in the study of the schizophrenia concept is laid bare. How broad should the definition of the concept be, and which approach is to be favored in studies on schizophrenia, far instance on physiological or biochemical correlates of this disarder, or on epidemiology, or treatment response? As long as there is no concensus on this matter (it may never be reached), two approaches seem appropriate. The first, categorical, approach consists of delimiting a central schizophrenic syndrome from one or more less typical syndromes, for instance a schizoaffective syndrome, an acute paychotic syndrome, and a borderline schizophrenic syndrome. The second one is chousing for a dimensional approach by scoring symptoms on severity scales or, most extreme, abandoning diagnostic categories and terminology altogether and chaosing for scoring of continuously diatributed personality characteristics.

The diagnostic approach confronts us with the self-reinforcing circular arguments used to establish categories, which on repeated application invest the latter with the appearance of possessing objective and definable reality" (Sokal and Sneath, 1963). This common 
practice ignores the fact that the existence of a diagnostic category as a natural group defined by certain characteristics has been assumed but not demonstrated. Validating evidence is thus required. It is often sought on other levels of anelysis. But since one of the prime purposes of classificatory schemes is to develop procedures whereby the constructs, categaries, and languages of one level can be related to other levels without a loss of reality at any level, advancement of our knowledge at other Levels, for instance the level of attention and information processing, is likely to necessitate continuing adaptations of categories based on the level of behavioral. disturbance.

The only alternative seems a numerical taxonomic approach (Sokal and Sneath, 1963; Sneath and Soka1, 1973). Numerical taxonomy has been developed because of an increasing dissatisfaction with the principles and practice of bialogical classification. But this approach need not be limited to biology, but may be fruitfully applied to other fields. Numerical taxonomy aims at the development. of naturally accurring empirically defined classes rather than categories based on a single level of description (such as psychiatric symptomatology), or even a single essential feature (which characterizes a monothetic classification). More accurate conceptualizations of reality can thus be arrived at. This approach is polythetic, which means that classification is based upon information from many levels, or systems of functioning, and that all measures are of equal value before classification. A polythetic arrangement places together individuals that have the greateet number of shared features, and no single feature is either essential to group membership or is sufficient to make an individual a member of the group. A formal definition was formulated by Beckner (1959): "A class is ordinarily defined by reference to a set of properties which are both necessary and sufficient (by stipulation) for membership in the class. It is possible, however, to define a group $k$ in terms of a set $G$ of properties $f_{1}, f_{2}, \ldots, f_{n}$ in a different manner. Suppose we have an aggregation of individuals (we shall not as yet call them a. 
class) such thet:

(1) Each one possesses a large (but unspecified) number of the properties of $\mathrm{G}$.

(2) Each $f$ in $G$ is possessed by large numbers of these individuals and

(3) No $f$ in $G$ is possessed by every individual in the aggregate. By the terms of (3), no ff is necessary for membership in this aggregate; and nothing has been said to either warrant or rule out the possiblity that some $f$ in $G$ is sufficient for membership in the aggregate. A class is considered polythetic if the first two conditions are fulfilled and is fully polythetic if the third condition is also fulfilled'. Although, according to this definition, the DSM-III definition of schizophrenia is related to polythetic classifications, it should be noted that the DSM-III characters are limited to the behavioral, cognitive and emotional level of functioning. In paychiatric polythetic classifications levels should be crossed, in order to cover as much information as possible, and the number of characters should probably be much larger than in DSM-III.

The basic positions or axioms of numerical taxonomy, as described by Sokal and Sneath (1963), are as follows:

(1) The ideal taxonomy is that in which the taxa have the greatest content of information and which is based on as many characters as possible.

(2) A priori, every character is of equal weight in creating natural taxa.

(3) Overall similarity (or affinity) between any two entities is a function of the similarity of the many characters in which they are being compared.

(4) Distinct taxa can be constructed because of diverse character correlations in the groups under study.

(5) Taxonomy as concelved by us is therefore a strictly empirical science.

(6) Affinity is estimated independently of phylagenetic 
considerations' .

The advantages of the polythetic classification strategy for psychiatry were discussed by Corning and Steffy (1979). The relevant, reliable and valid measures that are required for this approach can be sought, among athers, in the attention-information processing area, but it is essential that they cover a broad range of levels of functioning, from biochemical abnormalities to maladaptive social behavior. In the near future progress will be made in this area, and it seems probable that, at least in research areas, polythetic classifications will tend to replace current classifications, including the concept of schizophrenia. 


\subsection{THE CONCEPT OF ATTENTION.}

The term 'attention', although suggesting a unitary phenomenon, refers to a variety of partially overlapping concepts, each differing in meaning. The problem of attention is old, but had remained with the philosophers and not with the scientists, until in the first half of the nineteenth century Müller discussed in his theory of the specific energies of the nerves the selective power of the mind over against the specific energies (see Boring, 1929). William James, one of the first experimental psychologist, simply stated: "Everyone knows what attention is. It is the taking possession by the mind, in clear and vived form, of one out of what seem several simultaneously possible objects or trains of thought. Focalization, concentration, of consciousness are of its essence. It implies withdrawal from some things in order to deal effectively with others' (James, 1950). In his compilation of the early theories on attention, Pillsbury (1908) defined attention as 'an increased clearness and prominence of some one idea, sensation, or object, whether remembered or directly given from the external world, so that for the time it is made to constitute the most important feature of consciousness'. Dther early experimental psychologists, mainly introspectively oriented, were Wundt (1874) and Titchener (1908). Both also contributed to the then already controversial concept of attention. To Wundt attention was an 'inner activity', causing ideas to be present in consciousness to differing degrees. Titchener considered attention an 'intensive attribute' of a conscious experience. After these investigators came the rise of behavioriam, Gestalt psychology, and phenomenology, and for many years these schools have represented the prevailing paradigme of psychological research. The environment of the subject and observable behavior came into the centre of interest rather than the internal psychological processes. Although perception was the primary focus of interest for the Gestalt psychologists, they were not interested in the problem of attention. Also psychoanalytic theory, although 'internally' oriented, did not show itself interested in attention as a theoretical construct, but to some 
degree the psychoanalytic concept of cathexis, of investment of energy, may be considered related to the attention concept as a psychodynamic process.

Since the 1950's, however, the study of individual psychological processes was more and more stimulated by researchers applying newly developed theoretical models, derived from communication and information theory, to the study of the mind and, specifically, of attentional processes. Besides a new stimulating paradigm, new technology became available, including digital computers and other electronic systems. Soon the concept of attention became respectable again in the world of experimental psychology. New models of attention were developed, tested, and changed, and a new interest was awaked in the involvement of attentional processes in psychiatric disorders, as well as in other areas of human functioning. Modern coinitive psychology has accepted the concept of attention as stimulating. Man is no longer seen as a passive recipient of external stimuli, but as actively selecting and influencing his perceptions, and thereby in some respects creating his environment rather than being dependent on it. Human behavior cannot be predicted directly from the stimuli impinging on the organism, and attention describes the phenomenon of selective processing and responding.

The major shortcoming in the concept of attention lies in its overinclusiveness. The areas of cognitive psychology, information processing theory and psychophysiology have provided conceptually and methodologically sophisticated views on attention, and this has led many investigators to feel a need for subdividing this concept. Berlyne (1970), for instance, has distinguished between two principal meanings of the concept 'attention': intensive and selective phenomena. Among intensive aspects he distinguished attentiveness, concentration and arousal. Selective aspects are, in his opinion, selective attention, sbstraction and exploratory behaviar. Posner and co-workers (Posner and Boies, 1971; Posner, Klein, Summers and Buggie, 1973; Posner, 1975) have argued that alertness (arousal), selectivity 
(set) and processing capacity (consciousness) can be identified as separate components of attention. Both alertness and selectivity are supposed to affect the ratie at which a signal becomes available to later processing mechanisms, but in different ways. In their opinion, alertness reduces the time for information processing systems to respond to the buildup of information about the signal, while selectivity refers to increase in processing efficiency by activation of internal representations, which is information stored in memory systems. They suggest that consciousness refers to those mental operations which require the use of particular brain mechenisms of limited capacity, which implicates that interference is possible. Zubin (1975) attempted to clarify the problem of the definition of attention by distinguishing the following aspects: selection of the part of the environment for focusing, maintenance of the focus, and shift of the focus ta some other part of the environment. This relatively simple categorization in selective attention, maintenance or sustained attention, and switching attention has received some acceptance in the study of attention in schizophrenia.

Usually, when using the term attention, the selective aspects of information processing are referred to. Intensive aspects are more or less brought together in the concept of arousal. According to modern information processing theorists, attention is to be viewed as the control process in the passage of information through the information processing system (central nervous system) (e.g., Underwood, 1978). This concept of selective attention is intimately related to that of a limited capacity of the processing and memory systems. Selective attention would serve no purpose if processing capacity were not limited.

Modern theorizing on the concept of attention started with Broadbent in 1958, when he presented the first complete theory of attention. His model assumed a bottleneck (filter) at or just prior to the stage of perceptual analysis, so that but one channel of information at a time can be dealed with. In this theory selection among stimuli is made on 
the basis of physical characteristics of the information. Since then there have been disagreements, however, about the locus of selective attention in the hierarchy of processing of incoming sensory information. Later models favor late selection of incoming information. Experiments of Treisman (1964) showed that sensory oves alone were not a sufficient explanation of the mechanism of selection of relevant from irrelevant information. Her results suggested that features of incoming information are analyzed successively, by a sequence of operations, starting with general physical features and proceeding to semantic and grammatical features when that is necessary. In this model selection takes place during the analysis, and requires complex processing itself, using information stored in memory. In recent years much of the research on attention can be characterized as studies af tuo models (Norman, 1976). One model favors early selection of incoming information, the other favors late selection. Both models of the site of attentional restriction have received support, although there are also results not easily accountable by these models. In 1970 Broadbent identified two stages of selective attention: 'stimulus set", a stage in which the input from irrelevant channels is reduced, and 'response set", a stage of memory-based selection systems. Treisman (1969) even proposed four types of selection mechanisme: a selection of inputs or channels of sensory data, a selection of analyzers for examining specific stimulus features in more detail, a selection of targets for identifying specific stimulus configurations, and selection of outputs for deciding on memory entry or motor response. It is clear that the concept of attention is interwoven with conceptions of ather cognitive processes like memory, decision making and motor output, and that both cannot be divarced.

Therefore current theorizing on attention approeches the problem by pilacing the concept in theories of human information processing. Most of these theories have hypothesized that sensory information ie analyzed and transformed through a number of processing stages prior to the emission of selective responses. Incoming information is thought to be registered and stored in a sensory memory, called 
iconic (visual) or echolic (auditory) menory, from there the information is accessible during about one second. This information decays rapidly, or passes to ather processing stages of increasing complexity. Stages of short-term menory and af long-term memory have been postulated. Finally, higher cognitive systems are concerned vith decision making. In recent years, however, models of memory have changed and no longer postulate separate memory stores. Instead, the type of encoding and the degree of processing is considered decisive.

Norman and Bobrow (1975) do only make a distinction between resource-limited operations, when an increase in the amount of processing resources can result in improved performance, and datalimited operations, when performance is independent af processing resouroes, but is limited by the amount of incoming information. Norman (1976) claseifies sequences of operations that proceed from the incoming data, through increasingly complex analyses, as "data driven' or 'bottom-up' analyses. Operations that start at the level of conceptualizations of the incoming information (Iike expectations, goals and decisions) and work down are called 'conceptually driven" or 'top-down'. In humen information processing both systems of analysis are essential, and continually interacting. In humans the conceptually driven strategies are obviously of paramount importance. Probably both types of processing operate simultaneausly. As stated by Kietzman, Spring and Zubin (1980), the concept of attention represents a bridge between these two types of processing and helps to desoribe how the two systems operate in unison. The distinction batween data driven and conceptually driven operations has parallels with the distinction between involuntary and voluntary attention, or between automatic and controlled processing. The proces by which ane learns to attend and respond automatically to certains stimuli can be described as a gradual shifting from conceptually driven to data driven processing. This sthifting is of much significance as it frees procesaing capacity. Voluntary and consciously (controlled) selecting is a type of attentional activity that proceeds slowly, requires effort, and is highly demanding of processing capacity (Schneider and Shiffrin, 1977; Shiffrin and Schneider, 1977). In this modern view on 
attentional processes, the problem of the locus of attention, of the critical attentional bottleneck, has become irrelevant. There are limits on how much processing can be done at any one time, but these limits do no appear to be the result of fixed selection devices. Rather, the total available pracessing resources are limited, as first suggested by Kahneman (1973), as well as the rate of the serial. comparison processes, characteristic of controlled (voluntary) processing (Schneider and Shiffrin, 1977). According to this view, when effort or attentional resources are allocated to the analysis (controlled processing) of one information category, fewer resources are available to process ather kinds of information. The result is selectivity. On the other hand, invaluntary, automatic processing is learned in long-term store and does not require attention. The total resource capacity is considered to be limited, although variable, and related to the arousal system.

Arousal as a concept denotes the activation of the nervous system (electrocortical arousal), a process which is intimately linked with the stress response of the whole organism. The relation between attention and performance on a task and arousal is known as the Yerkes-Dodson law, which states that performance is an inverse U-shaped function of arousal level (Verkes and Dodson, 1908). Under and over aroused subjects perform poorer, the former probably because of a relative lack of motivation, the latter as the result of an increasingly narrowing of attention, which eventually proves detrimental. This explanation of the decrenent of task performance with increasing arousal, a decrement which occurs sooner in complex tasks than in simple ones, is known as Easterbrook's hypothesis (Easterbrook, 1959). It supposes a gradually narrowing of attention, and a simultaneous impaired ability to discriminate relevant from irrelevant stimuli. In recent years the Yerkes-Dodson law has received criticism because of its aversimplification. Hockey (1979), for instance, has concluded that arousal changes the balance of the effectiveness of cognitive resources, rather than causing a global shift. 
The consideration of mental resources adds a unitary concept to the nature of attention. Processing resources can be directed in many different ways, from preparing to and processing of sensory input to processing of internally generated programs. Attentional performance (controlled processing) requires effort, and this idea is the link between the concepts of processing resources and arousal. In this way the concept of attention is linked with the information processing capacity and efficiency of the central nervous system as a whole. Dysfunctions of attentional processes, whether measured by the continuous performance test, reaction time, or other attentiondemanding tasks, may refer, according to this view, to dysfunctions anywhere in the controlled information processing systems of the central nervous system. 


\subsection{GENERAL ASPECTS OF ATTENTIONAL DYSFUNCTIONING IN SCHIZOPHRENIA.}

Nowhere in the field of functional psychiatric disorders are disturbances of attention and information processing more prominent than in the psychoses. Defects of attention in schizophrenia have been recorded since the schizophrenia concept was founded: "Schwere Störungen pflegt (...) regelmässig die Aufmerksamkeit zu zeigen. Wenn man auch oft die Kranken vorübergehend zum Aufpassen bringen kann, so besteht doch dabei nicht selten grosse Ablenkbarkeit, die ein längeres. Festhalten bei demselben Gegenstände unmöglich macht" (Kraepelin, 1904). Bleuler, in his classic volume 'Dementia praecox oder Gruppe der Schizophrenien' (1911), stated: 'Als Teilerscheinung der Affektivität leidet mit dieser auch die Aufmerkgamkeit. Soweit Interesse vorhanden ist - also in den leichteren Fallen für die Mehrzahl der Erlebnisse und in den schweren für gefühlsbetonte Tätigkeiten (...) - erscheint zwar die Aufmerksamkeit unseren jetzigen Beobachtungsmethoden normal. Wo aber der Affekt fehlt, mangelt auch der Trieb, den äusseren und inneren Vorgängen zu folgen und die Richtung der Sinne und der Gedanken zu dirigieren, d.h. die aktive Aufmerksamkeit. Ganz anders wird die passive Aufmerksamkeit alteriert: Es ist zwar selbstverständlich, dass interesselosen oder autistisch abgekapselten Patienten die Aussenvelt sehr wenig beachten. Daneben aber wird merkwürdig viel von den Ereignissen registriert, um die sich die Patienten nicht kimmern. Die Auslese, die die normale Aufmerksamkeit unter den Sinneseindrücken trifft, kann bis auf Null herabgesetzt sein, zo dass fast alles registriert wird, was den Sinnen zugeht. Die bahnende wie die hemmende Eigenschaft der Aufmerksamkeit ist also in gleicher Weise gestört'. Pillsbury (1980), in an early review of research on attention, concluded that 'the degenerations of the mind are nearly all accompanied by weakened or deranged attention".

Many modern investigators explain the heterogeneity of symptomatology in schizophrenia as the result of differing adaptations to basically 
similar deficits of attention or, more generally, of information processing. In 1961 McGhie and Chapman published an interview study of schizophrenic's subjective experience of formal disorder in cognitive function. Their subjects were 'early schizophrenic patients, where the subsequent caurse of the iliness confirmed the original diagnosis'. The investigators reported selected quotations from the remarks of the patients to illustrate their theory of decreased selective attention and increased distractibility in schizophrenia. Some of these statements serve as good illustrations of the disturbances of attention in schizophrenic patients. For instance: 'It's as if I am too wide avake - very, very alert. I can't relax at all. Everything seems to go through me. I just can't shut things out'. Or: "Things axe coming in too fast. I lose my grip of it and get lost. I am attending to everything at once and as a result I do not really attend to anything". An example of disturbance of information processing at the level of speech perception: 'Sometimes when people speak to me my head is overloaded. It "s too much to hold at once. It goes out as quick as it goes in. It makes you forget what you just heard because you can't get hearing it long enough. It's just words in the air unless you can figure it out from their faces'. This last statement makes clear how additional information, following ather pathways which, in this case, are probably even mainly localized in the other cerebral hemisphere, may help identifying the relevant aspects of the message. Probably the increased redundancy in information lessens the effects of interfering irrelevant input in the information processing systems. When the speech analysing systems are at their processing limits, only additional channels, designed to process other types of incoming information, can provide the necessary extension of processing resources.

In her autobiographical account Norma MacDonald (1960) attributed her psychatic experiences to a 'mental filter' breakdoun: "Now, many years later, I can appreciate what had happened. Each of us is capable of coping with a large number of stimuli, invading our being through any one of the senses. We could hear every sound yithin earshot and see every object, line, and colour within the field of 
vision, and so on. It's obvious that we would be incapable of carrying on any of our daily activities if even one-hundredth of all these available stimuli invaded us at once. So the mind must have a filter which functions without our conscious thought, sorting stivuli and allowing only those which are relevant to the situation in hand to disturb consciousness. And this filter must be working at maximum efficiency at all times, particularly when we require a high degree of concentration. What had happened to me in Toronto was a breakdown in the filter, and a hodge-podge of unrelated stimuli were distracting me from things which should have had my undivided attention $(\ldots)$. My brain, after a short time, became sore with a real physical soreness, as if it had been rubbed with sandpaper until it was raw. It felt like a bleeding sponge (...). I had very little ability to sort the relevant from the irrelevant. The filter had broken down. Completely unrelated events became intricately connected in my mind".

Freedman and Chapman (1973) interviewed schizophrenic subjects (classified by independent judges on the basis of the current and Past Psychopathology Scale Interview) wing standard questions, derived from published autobiographical boaks and articles by schizophrenics. (These information sources are included in the references of Freedman, 1974). They found that about half of their subjects experienced attentional deficits like thase described by McGhie and Chapmen (1961). Although according to their findings not universal among schizophrenics, they conclude that deficits in focusing attention seem to be important. in a large category of schizophrenic patients. Grosa and Huber (1972) reported that 15 per cent of a total of 757 schizophrenic patients observed during 10 years reported spontaneously on perceptual disturbances (related to attentional dysfunctioning). Because of the dependence on spontaneous communications, this is likely to be an underestimation of the real prevalence.

The construct of attention has been used to interpret many types of deficits shown by schizophrenic patients in experimental situations. Investigative areas with which attentional dysfunctions have been associated are, according to, Garmezy (1977): perception research 
(disruption of size constancy, perceptual scanning, two-flash threshold), cognition research (overinclusiveness, memory deficits, language deficits, word association disturbances), motoric behavior (response inhibition, smooth pursuit eye movements), attentional tasks (span of apprehension, backward masking, reaction time tasks, distraction effects, continuous performance test), psychophysiological research (skin conductance response, heart rate pupil size reactivity), neurophyaiological research (EEG activity, evoked potentials, contingent negative variation), psychopharmacology (response to phenothiazines). Garmezy concludes 'that defective attention has profound consequences that extend over a wide band of behavioral domains in schizophrenia with such defects providing a basis for the patient's failure to acquire competence skills of a cognitive, affective, motivational and social-interactional nature'. The relevant literature provides much evidence that these attentional deficits are primarily related to the vulnerability to schizophrenia and schizophrenia-like disorders, rather than being the result of the state of psychosis. Attention dysfunction is thus considered a relatively stable trait and an antecedent to rather than a secondary consequence of schizophrenic psychotic episades. Nevertheless, attention and information processing variables may in addition function as episode markers by reflecting the degree of disorganization during a psychotic episode. There is evidence that those attentional and information processing deficits that are operationalized by some modern methods, notably the continuous performance test, reaction time redundancy deficit and pursuit eye tracking, are primarily related to vulnerability. This evidence is discussed in the sections were these tests are presented and the literature ia surveyed (Chapter 5.2.).

The concept of vulnerability to schizophrenia, as a relatively permanent, enduring trait, has been touched on by many investigators. Zubin and Spring (1977) introduced vulnerability as a "second-order" model, proposed as the common denominator, connecting the many models that currently characterize the field of research in schizophrenia. Their vulnerability madel proposes that everyone is endowed with a 
degree of vulnerability that under suitable circumstances will express itself in an episode of schizophrenic illness. The various etiological models offer suggestions about the possible origines of this vulnerability. The importance of vulnerability indicators, among which measures of attentional deficit are supposed to rank high, is in their fundamental contribution to the study of schizophrenia and related disorders. They can be useful in the problem of classification. Other promising research areas for vulnerability indicators are the genetics of schizophrenia and related disorders, and the study of changes in response to treatment. In the future it may, on the basis of our knowledge of vulnerability indicators, even be possible to open new perspectives on the early detection and probably prevention of psychiatric disturbance.

For the study of vulnerability concept it is interesting, of course, to investigate the interrelationships between attention and information processing measures that are supposed to relate to vulnerability. Generally these interrelationships have not yet received the attention they deserve. Among studies comparing different attention tasks within samples of schizophrenic subjects are the following.

Kopfstein and Neale (1972) published a study of the performance of schizophrenic and nonschizophrenic, acute and chronic, psychiatric patients on five tests purported to measure attention dysfunction. The tasks in this study were: Reaction time, Size Estimation (a perception test), Proverb Interpretation and Object Sorting (both considered an index of overinclusion) and Vigilance (a test of sustained attention). Contrary to assumptions, these tests were found not to intercorrelate highly. In a subsequient study of these investigators (Kopfatein and Neale, 1973) a different strategy was adopted, in which groups of paychiatric patients (clinically diagnosed as schizophrenic or nonschizophrenic) were formed having similar performance profiles on the five tasks. These groups were examined to identify individual difference variables which distinguished among them, but little resemblance was found between the groups thus formed and usually 
accepted clinical categories (e.g., schizophrenic vs. nonachizophrenic, acute vs. chronic, paranoid vs. non-paranoidy. However, the diagnosis of schizophrenia was the best variable for differentiating between good and poor performance on the laboratory taske.

Asarnow and MacGirimon (1978) compared the performance of acute achizophrenics, remitted schizophrenics and normal contrals on two attention tasks: the Continuous Performance Test and a visual search task uithin a span of apprehension paradigm. Although both patient groups performed worae than the controls on both tasks, the correlational data revealed that these tasks shared little common variance. Kornetsky and Orzack (1978) found a significantly slower reaction time in poor performers on the Continuous Performance Test as compared to gaod performers. No relation between reaction time and smooth pursuit eye tracking was found by Pivik (1979), although both differentilated between patient and normal control graups. Pass, Klorman, Salzman, et al. (1980) reported a lack of significant correlations between Continuous Performance Test score and amplitude of the late positive component ( $P$ 300) of the evoked potential to the critical and the noncritical stimuli in groups of schizophrenic and nonschizophrenic patients as well as of normal controls.

The results of studies like those mentioned here highlight the heterogeneous nature of the attention concept. The low correlations between the attention tests have to be attributed to differences in the processing requirements. These results suggest that the attentional deficit of achizophrenic patients has also a non-unitary character. This conclusion is not unexpected, of course.

In studies of attention and information processing in schizophrenia the related concept of arousal often cannot be avolided. As already discussed, the relation between both concepts is usually described by referxing to the Yerkes-Dodson law. However, the heterogeneous nature of the arousal concept is well known. In the case of schizophrenia the necessity to postulate more than one arousal system has been 
stressed, for instance, by Lapidus and Schmolling (1975). The heterogeneity of the attention concept and of attentional deficits in schizophrenics has already been mentioned. The varying and partially overlapping definitions of these concepts further complicate a discussion about which is to be considered as the primary defect in schizophrenia: attention - information processing or arousal. (Does the schizophrenic experiencing informetion-processing difficulties become aroused, or does the arousal disorder create the difficulty in processing? - Cromwell, 1978). This discussion does not seem to be very important, however. As mentioned in the previous section, electrocortical arousal determines the availability of information processing resources and the efficiency of selective processes. The concepts of arousal and selective attention are intimately linked, and one cannot be separated from the other. Both constitute one complex system and electrocortical arousal (and general stress responses) will increase in proportion to the amount of information to be processed per unit time (Warburton, 1979 a; b).

In sumnary, it is assumed here that measures of attention and information processing deficits are rightly considered promising indicators of very basic veaknesses in the organization of central nervous system functions, conceptualized as vulnerability. Many investigators have based theories of schizophrenia on defects in attention and information processing. A number of these theories gets attention in the next section. 


\subsection{MODELS OF SCHIZOPHRENIA BASED ON DEFECTS OF ATTENTION AND}

INFORMATION PROCESSING.

A limited survey of recent theories of schizophrenia, based on information processing models, is presented here. It illustrates the capacity and the attractiveness of these models in generating theories and hypotheses about the complex concept of schizophrenia. These models have in common that they approach the symptons and behavioral deviancies of schizophrenia by attributing these to fundamental disturbences in attention and information processing.

\section{Input dysfunction (Venables).}

Venables (1964, discussion in Epstein and Coleman, 1970 and Neale and Cromvell, 1970) has proposed that schizophrenic patients are suffering from an 'input dysfunction'. According to this theory acute schizophrenics have an excessively broad attention and a low but variable level of arousal, whereas chronic schizophrenics have excessively narrow attention and a high level of arousal. The chronic overarousal in chronic patient could be brought about by a failure of inhibitory processes (Venables, 1966). 'Chronic schizophrenics - and possibly included in this category are process patients - tend to be characterized by a state of restriction of the attentional field resulting from elevated states of sympathetic and cortical activation (...). In contrast to the chronic patient, the acute (and possibly the reactive and paranoid) patient is characterized by an inability to restrict the range of his attention so he is fllooded by sensory impressions from all quarters (...). The acute patient"s broadened level of attention would appear to arise from a law level of cortical activation or poseibly the parasympathetic imbalance which he displays' (Venables, 1964). The hypothesis of narrowing and broadening of attention follows from the relationship between arousal and attention as stated by the theory of Easterbrook (1959), which proposes an increased selectivity of attention under high arousal. The theory of Venables is rather crude, but it takes into account 
differences among the group of schizophrenic patients, like some of the other theories indeed.

\section{Defective scanning control and field articulation (Silverman).}

Silverman (1964, discussion in McGhie, 1970 and Neale and Cromwell, 1970) has applied cognitive control principles to the atudy of attention in schizophrenia. Two cognitive strategies considered to be important are scanning control, which reflects differences in the extensiveness with which stimuli are sampled when attention is directed to part of the environment, and field articulation, which refers to the articulation of the sampled cues into relevant and irrelevant aspects, the inhibition of those aspects that are not essential.

Silverman too makes a distinction among the group af schizophrenic patients. Extensive scanning and high field articulation scores are associated with the paranoid and good premorbid subtype, whereas minimal scanning and undifferentiated and poor field articulation are associated with nonparanoid and poor premorbid schizophrenics. A moderate or nonextreme attention deployment response pattern is associated with a good prognasis. Chronicity is associated with a tendency to reversion of scanning response style. The scanning style of the good premorbid paranoid patient is supposed to serve as an actively monitoring out stimuli, a defence mechanism.

\section{Filter breakdaun (McGhie).}

McGhie and his colleagues (McGhie and Chapman, 1961; McGhie, Chapman and Lawson, 1965; Lawaon, McGhie and Chapman, 1966; McGhie, 1970) have presented a theory of schizophrenia based on a breakdoun of normal filtering processes. These investigators have derived their theory from studies of the effects of distraction on various aspects of schizophrenic performance. The theory is based on the model of the limited capacity decision channel as described by Broadbent (1958). Overloading of this channel will be likely when range of atimuli 
has to be monitored and complex decisions are asked for. The consequence is a breakdown in performance. Hence schizophrenic patients are lese able to attend selectively and to process anly relevant information.

The effect of this impairment on performance will vary with a number of factore, including the amount of information to be processed, the modality in which the information is presented, and the rate of arrival of Information. All this concerns 'process' schizophrenic subjecta. Paranoid gchizophrenics, in the opinion of McGhie (1970), demonstrate a highly selective type of attention by which they screen out extraneous stimulation more efficiently, but also more crudely, than normal subjects. It is suggested that any differences which do exist between acute and chronic patients reflect various behavioral strategies adopted by the chronic schizophrenics to reduce the flow of stimulation to a more tolerable level.

\section{Slowness of information processing (Vates).}

Yates (1966) has suggested that the primary deficit in schizophrenia lies in the abnormally slow rate of information processing. Since the short-term memory system can hold information only for a short time, the amount of stored information lost per until time will be greater than in normals. As a result only part of the relevant information is succesfully processed, which explains the schizophrenic patient's confusion. The higher brainfunctions are not given sufficient information to make appropriate decisions. Yates denies the relevance of the distracting effects of irrelevant stimulation. His theory also rejects an impaired filtering mechaniem. Emphasis is placed an the response part of the information processing chain by the influence of the response rate on the overload in short-term memory.

\section{Qverinclugion (Payne).}

Payne and his ce-workers (Payne and Hewlett, 1960; Payne, 1966) have suggested that the overinclusive thinking, an inability to preserve 
conceptual boundaries, found in many schizaphrenic patients, is the result of a defect of a filter mechanism. This hypothetical filter normally serves to screen out irrelevant stimuli and thoughts. The filter defect results in thinking becoming distracted by external. events and by irrelevant personal thoughts and emotions. The result is that 'Selective perception becomes impossible so that ingtead of dealing with the essence of the problem, irrelevant aspects are perceived and thought about...' (Payne, 1966). Payne's studies suggested that overinclusive thinking, as measured by his tests, is one aspect of a general deficit of selective attentiom which is more evident in acutely ill patients of the paranoid subtype (Molhie, 1970). His attention based theory is clearly related to McGinie's. Initially overinclusion was thought to be specific to schizophrenic patients. However, later Payne has changled his position, and concluded that, "in spite of a very considerable amount of research, overinclusive thinking remains a somewhat elusive concept" (Payne, 1971). He even concluded that it seems an uncommon phenomenon in schizophrenia and not specific to this disorder, even not especially associated with delusional thinking, but perhaps implying a better prognosis. So at the moment the usefulness of the concept of overinclusive thinking for the study of schizophrenia seems questionable.

\section{Response interference (Broen).}

In the opinion of Broen (1968, Broen and Storms, 1966; 1967) there is no problem for schizaphrenic patients in sampling input. He auggests that schizophrenic behavior is characterized by response interference, by which term he means disorganized fluctuations among appropriate and competing responses. "According to the theory, the type of response interference that is important in schizophrenia results from a partial collapse of response hierarchies. Dominant and competing responses have the same hierarchical order as in normals, but the strenghts of the different response tendencies are closer together in schizophrenics, and the renote associates, the interfering attention responses, and ather error tendencies that 
occasionally occur in nornals are exacerbated in schizophrenics (...), response hierarchies collapse when the strength of dominant responses is restricted by lower verage response-strength ceiling in schizophrenics. The collaps will be exacerbated by increased arousal. which, with dominant responses at ceiling, acts only to multiply the strengthe of competing responses' (Broen and Storms, 1967). The result is 'randomized' behavior and disorganization.

According to Broen (1966) chronic schizophrenics reduce the ir disorganization by reducing the range and speed of cue utilization ('gcanning'). In this respect Broen"'s model integrates those of Silverman and Venables. Generally it is a parallel in terms of response selection to the stimulus selection models. Broen's initial assumption of response interference as specific to schizophrenia has later been subjected to serious criticism. Response interference appears to be exhibited by most subjects under states of heightened arousal (Depue, 1974). Perhaps the theory is at its best when accounting for cognitive disorganization produced by high levels of arousal and response competition (Epstein and Coleman, 1970).

\section{All-or-none inhibitory control (Epstein).}

Epstein (1967; Epetein and Coleman, 1970) has hypothesized an inadequately modulated inhibitory system for controlling excitation as the basic defect in schizophrenia. '(...) it is assumed that the schizophrenic has a crude all-or-none system of inhibitory controls, so that he is unable to vary his inner and overt reactions to corraspond closely with the requirements of variations in internal and externel stimulation. He thus tends to respond either too strongly or not enough, and many vary from one extreme to the other" (Epstein, 1967). As the problem concerns the modulation of excitation, it wil1 more easily be manifested at high magnitudes of stimulation. In the opinion of Epstein (Epstein and Coleman, 1970) it follows from the all-or-none inhibitary system for reacting to stimulus inputs that schizophrenics must suffer from an attention defect, because effective attention requires a fine-tuned inhibitory system. In this theory 
attention in the schizophrenic patient will be excessively broad (absence of selective inhibition) or excessively narrow (massive inhibition). The cognitive deficits are deduced from the attention defect.

\section{Segmental set (Shakow).}

Shakou (1962; 1963; 1971) has introduced the concept of segmental set as an explanation of certain findings in reaction time research (see Chapter 5.2.2.) In his view the schizophrenic patient, when performing a task, is subject to interference from aspects of his internal and external environment. He has difficulty to meintain a 'major set' by which Shakow means 'difficulty in keeping up a state of readiness for response to a coming stimulus, the state which facilitates the optimal response called for by a given aituation" (Shakow, 1962). There is a constant intrusion of 'minor sets', irrelevant associations arising from three sources: chance distractors from the environment, irrelevancies from the stimulus situation, and irrelevancies from pas experience. The internal and the external environment of the schizophrenic patient are segmentalized. "It is this concept of segnental set which appears to me to have value for the understanding of much of the formal deficit which reveals itself in chronic schizophrenia' (Shakow, 1962). Obviously this concept of Shakow is related to the selective attention dysfunction concept of other investigators. As Garmezy (1978) has remarked, Shakow's formulation is in addition related to the response interference hypothesis and to the concept of stimulus overload.

\section{Crassmadel retardation (Zubin).}

Zubin (1975) has suggested that the basic aspect of attention in which some schizophrenics deviate from other psychiatric patients is shift of attention, especially when it is externally controlled. In shifting attention from one stimulus modality to another schizophrenics should show greater retardation then normals. The 
explanation proposed by Zubin postulates that the immediate effect of a stimulus persists longer in the case of schizophrenics. Stimulation in one sensory modality shifts the balance of neural facilitation and inhibition for subsequent stimulation in another sensory modality. The 'crossmodal retardation' might lead to more difficulty in rapid scanning of the environment and, hence, to narrowed scanning.

\section{Vulnerability (Zubin and Spring).}

The 'oecond-arder' model proposed by Zubin and Spring (1977; Spring and Zubin, 1978), vulnerability, has already been introduced earlier in thig chapter. It is conceptualized as the common denominator of the many etiological models of schizophrenia, and as representing a persistent characteristic of an individual. In contrast, episodes of schizophrenic disorder are waxing and waning states. Put otherwise: vulnerability and episode stand in a trait-state relation. The vulnerability concept implies that each individual is endowed with a degree of vulnerability that under suitable circumstances will express itself in an episode of schizophrenic disturbance. An important implication of this model is the search for markers of vulnerability and of episodes of schizophrenic disorder. Those indexes that characterize the person before, during, and after an episode may turn out to be vulnerability markers, whereas episode markers characterize the person only during the episode.

In line with the vulnerability model of Zubin are Marshall's (1973) remarks: In choosing either an attentional or a response selection model as a basis for understanding schizophrenic's problems we necessarily restrict our view. Schizophrenics may experience difficulties in either or both stimulus analyzing or response aelection. They may also have difficulties in holding material in the short term memory mechanism, in retrieving memorial information, in transferring information within the system, or, in line with bleuler"s (1911) suggestion, in associating material at some central level in the system'. 
Each of the various information processing models (some of which are based on a currently outmoded madel of information processing, namely Broadbent's 1958 model) tap possible deficits in information

processing. It is very unlikely that there should be one basic defect in schizophrenia. The psychophysiological basis of schizophrenia seems to lie in the way the central nervous system is organized rather than in any single disturbance of function, a view that has, for instance, been defended by Claridge (1972). In recent years the functional asymmetry of the brain has received increasing interest. There is much evidence that the left hemisphere is more specialized for analytic, sequential processing, and the right hemisphere for holistic, spatial, parallel processing. Applying this knowledge to the study of schizophrenia, the hypothesis has been suggested that schizophrenic patients display left hemispheric impairment of function (e.g., Cruzelier and Flor-Henry, 1979; Newlin, Carpenter and Golden, 1981). This hypothesis thus localizes the information processing deficits suggested by models like those presented here, although in a rather crude way.

In summary, most. theories are one-sided, unspecific, and limited in scope, but usually they suggest that in schizophrenia data driven processing mechanisms are deficient, and that consequently conceptually driven processing is necessary to a much larger degree than in normal subjects. Data driven processing needs efficient automatic control mechanisms and when these are failing more complex operations are required, demanding more processing capacity and proceeding slower. The result is that attentional functioning (controlled processing) is deficient in schizophrenic patients. The slowness and inefficiency of processing, in turn, leads to adjustment. processes, because of the information input overload which may be the result (Miller, 1978). Input averload, of course, depends heavily on the characteristics of the environment. Failing adjustment processes result eventually in psychotic breakdown. The considerable variability in psychotic disturbance is considered to represent 
Equre 1.

From vulnerability to (Bchizophrenic) psychosis:

the developmental chain.

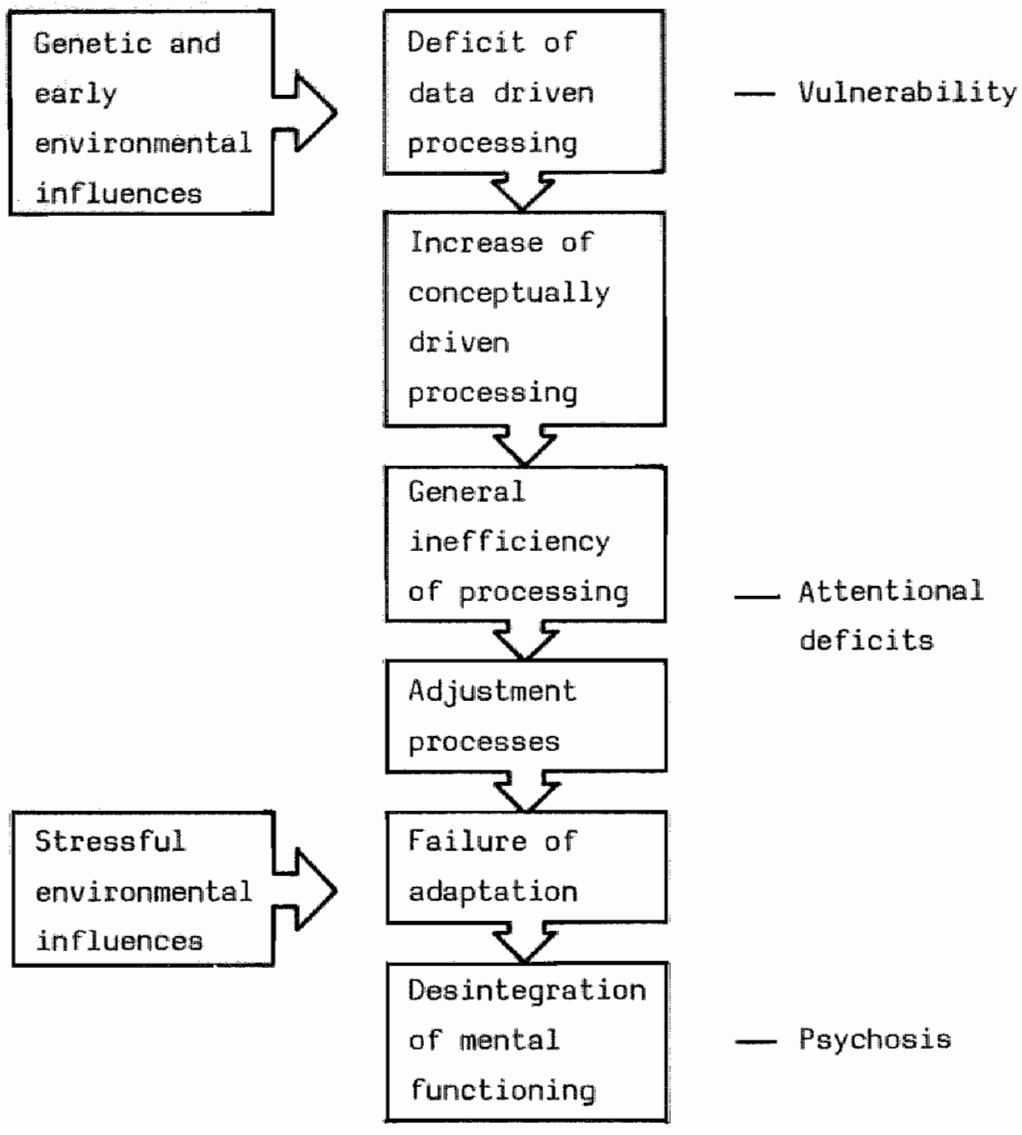


differing adaptations to the basic functional deficits (e.g*, Hemsley, 1977). Whether the insufficient control mechanisms are functions of wired-in structures or the result of deficient learning experiences: is another question. The evidence to date points in both directions.

The schema in Figure 1 presents an information processing model of schizophrenic psychosis which integrates the mast important aspects of current theorizing, as surveyed here. 
AIMS OF THE INUESTIGATION

This investigation explores the potentialities of some global measures of attention (controlled information processing) as objective indicators of psychiatric disturbance, especially of those types of disturbance which are clinically recognized as schizophrenia and related disorders. The attentional measures are compared in this respect with some more or less traditional (dimensional) diagnostic measures. The central question posed here is: Are attentional deficits (as operationalized by some attention tasks) indicative of the schizophrenia concept, or rather of psychotic disorders in general, or perhaps of serious psychiatric disturbance in general, whether psychotic or not? Additional interest centres on the comparison, regarding indication of severe (psychotic) disturbance, of measures of attentional deficit with measures of traditional characteristics of the schizophrenia concept, and measures of personality traits supposed to be related to psychosis. They also represent alternatives to traditional categorical classification.

The study of the epidemiology and of the genetics of mental disorders, of biochemical correlates of psychiatric disturbance, of psychopharmacology, but also of disordered communication processes, to mention some important areas, are all in need of more objective measures of psychopathology than are offered by the traditional diagnostic classifications. Description of psychiatric disturbance at the most complex level, namely the level of cognitions, emotions and social behavior seems insufficient as to the purposes of research 
areas like those just mentioned. Although traditional diagnostic criteria are constantly being refined, and progress will undoubtedly be made in the near future, a better perspective seems to measures of individual dysfunctioning at a more elementary level of analysis. The level of cerebral information processing and attentional processes offers such measures.

This study thus confines itself to measures of individual dysfunctioning. Of course many, and perhaps most, problems in the field of psychiatry are concerned with distorted interpersonal relationships and are linked with social problems. The systems approach in description and therapy should be acknowledged as a major advance, compared with the purely disease-oriented approaches of the early days of psychiatry. It is no alternative, however, to the description and therapy of psychiatric disturbance in terms of individual dysfunctioning. It is an addition, at another level of analysis. Moreover, the study of the relevance of these different orientations can only advance when reliable indicators of dysfunction at each of these levels of description are available. The study of interpersonal factors, of pathological communication, of distorted family patterns, etc., is heavily dependent on the availability of reliable measures of individual dysfunctioning. Only then can the contribution of supra-individual systems pathology be determined. It is worth noting that attentional mechanisms are to a large degree a bridge between internal and external 'realities'. Attentional measures are not really "pure organismic" parameters in this respect.

There seems to be a paradox in the aims of investigations like this: while the search is for objective indicators of psychiatric disturbance, these indicators are usulally validated by studying the correlations with existing classifications, that describe their objects of study at the most complex level, the level of behavior, cognitions, and emotions. However, the higher the correlations found between objective tests and traditional diagnostic classifications, the less the need for these new develapments. An the other hand, low correlations with traditional diagnostic classification does not 
preclude the usefulness of a classification based on ane or more objective measures. Validation may be sought, for instance, in predictive power as to course and outcome of a psychotic episode, or in (neuroleptic) treatment response, or in the degree of genetic transmission. It may also be sought in its persistence in spite of a varying clinical condition, that is, in its association with wulnerability rather than current state. Research studying these types of relationship has been done, although rather sparingly, and is still proceeding. Nevertheless, the study of correlations of objective measures with psychiatric diagnosis remains important. In particular, the explaining capacity of combinations of objective measures as to the variance in clinical psychopathological dysfunctioning is worth investigation. Disturbance of behavior, cognitions, and emotions will remain the domain where psychiatric problems are identified. The link with this level of description is therefore crucial to the viability of any classificatory system. For research purposes, polythetic classification strategies are likely to develop and to replace traditional classifications. In these new classifications attentional and information processing variables will certainly find a place, in addition to measures from other levels of analysis.

In this investigation subjects from five diagnostic categories are studied. These categories, defined according to DSM-III, are: normal, neurosis, schizotypal personality disorder, non-schizophrenic psychosis, schizophrenia. In addition to categorical diagnostic classification subjects receive dimensional diagnostic scores on three symptom and social functioning rating scales relevant to the schizophrenia concept, regarding Affective Flattening, Sacial. Isolation and Premorbid Asaciality. They get also scores on the selfrating scales of the Eysenck Personality Questionnaire, in particuler on the Psychoticism scale. This scale represents a personality dimension postulated to be related to the predisposition to develop a psychotic disorder. Attentional performance of the subjects is measured by a set of attentional tasks. These controlled information processing tasks are: 5mooth Pursuit Eye Movements (Eye Tracking), Reaction Time - Mean and Redundancy Deficit, Continuous Performance 
Test, and Contingent Negative Variation. The diagnostic and the attentionel variables are described in detail in Chapter 5. 
The present investigation thus studies the relations between measures of attention (controlled information processing) and psychiatric diagnosis. The attentional measures are compared in this respect with some dimensional (clinical and psychometric) diagnostic measures. The attentional measures are on the level of performance as vell as on the level of electrophysiological processes in the brain. Also analysed are the interrelationships between the various attentional measures and between the dimensional diagnostic measures. These analyses have to be viewed in the context of a search for more sophisticated indicators of psychiatric disturbance, especially (schizophrenic) psychosis, than are offered by traditional diagnostic descriptions. This taxonomic aspect is the primary interest of this study.

It is assumed that each of the measures of attention in this investigation has the potential to distinguish between good and poor attentional performers. The literature, to be discussed in the next chapter, supports this assumption. When the attentional deficit of psychosis is conceptualized as a unitary construct and these measures are supposed to be highly specific to this construct it follows that these measures should share much common variance and, therefore, that there should be a considerable overlap between the groups of poor performers as distinguished by these different methods. This would be an 'ideal' situation that is, of course, unlikely to occur. Rather, a more limited degree of shared variance seems probable, as attention 
and vulnerability are by no means unitary constructs and as the selected tests are mutually differing in their processing requirements.

Attention or controlled information processing is assessed here at the level of performance by three tasks: Smooth Pursuit Eye Movements (Eye Tracking), Reaction Time (Mean and Redundancy Deficit), and Continuous Performance Test, and at the electrophysiological level by the Comtingent Negative Variation. The controlled processing deficits, operationalized by these tasks, may be the result of genetically determined structural characteristics as well as of functional characteristics developed in the course of early and later experiences in life. Both cannot be separated easily. Camparable end results may have followed different developmental courses, so that different genetic lay-outs and a variety of environmental influences can result in a pseudo-unitary vulnerability. To some degree diverging results on attention and information processing tasks are thus likely for persons supposed to share with each other their heterageneaus vulnerability. It seems unlikely that there would be hardly any structure in this heterogeneity. Some clustering may be expected, and it is worth searching for these natural groups.

Vulnerability to schizophrenic psychosis is conceptualized as a trait not restricted to patients exhibiting or having exhibited clear schizophrenic psychotic symptoms. It is supposed also to be present in other psychiatric patients and in non-patients, although to a lesser degree. There seems no reason, therefore, to assume that the interrelationships between quality of performance on the various vulnerability-related tasks in this investigation should be essentially different in non-schizophrenic patients and in normal subjects, as compared to schizophrenic patients. The difference in level of analysis between performance and electrophysiological. measures of attention suggests that the intercorrelations between both sets of variables might be less high than the intercorrelations within the sets, but this is entirely conjectural. To summarize these considerations: it is assumed that the attentional measures in this 
investigation share a moderately high degree of common variance.

In outline, there are in this investigation five major diagnostic approaches to be recognized, four of which are at the level of clinical description. The first of these is the schizophrenie concept as a categorical construct. It is operationalized by the DSM-III criteria for schizophrenia and for schizotypal personality disorder, and to a somewhat lesser degree also by the criteria for a nonschizophrenic psychotic disorder. Although a fairly clear demarcation between the schizophrenic and non-schizophrenic psychotic categories is suggested by their sets of criteria, it should be remembered that DSM-III represents anly the latest development of the schizophrenia concept, and is by no means the definite answer to the problem of the demarcation of this diagnostic category. The non-schizophrenic psychotic category in this investigation does certainly contain many subjects who would have been classified schizophrenic by a number of earlier definitions of this concept, which is especially clear in view of the fact that patients with primary major depressive or manic disorder were excluded from this investigation and thus are not included in the non-schizophrenic psychotic category.

Put otheruise: the schizophrenic graup in this investigation represents psychotic patients labeled schizophrenic sccording to the latest criteria. Both psychotic groups together roughly represent those psychatic patients labeled schizophrenic in the past, especially when the traditional broad American approach to this diagnosis was still flourishing. Finally, the subjects of both psychotic groups plus the schizotypel patients probably represent more or less those (psychotic and non-psychotic) patients labeled schizophrenic by thase psychiatrists in the past who were supporter of the most broad, mainly paychodynamically oriented, approach to the schizophrenia concept. Many advocates of the schizophrenia spectrum concept assume a close relation between achizophrenia and schizotypal personality disorder, genetically determined, and assume that other types of psychosis are not part of this spectrum. However, not genetics but deficits of attention are in the focus of interegt here. 
Moreaver, as already indicated, the demarcation of schizophrenia as a diagnoatic category is uncertain and controveraial.

The second major diagnostic approach in this investigation is the severity of peychiatric diagnosio. Because schizophrenia is defined by DSM-III on the basis of signs of the illness being present for at least six monthe, while this criterion is not applied to the nonschizophrenic psychatic category, it seems justified to consider the schizophrenic category the most severely disturbed patient group. The other psychotic group comes next. of both non-psychotic patient groups, the schizatypal patients, by virtue of their hypothesized relation with the schizophrenic paychotic syndrome, are considered to represent the most disturbed category. The rank order schizophrenia non-schizophrenic poychosis - schizotypal personality disorder neurosis - normal then follows.

The third major diagnostic approach is represented by the variables Affective Flattening, Social Isolation and Premorbid Asociality. To the extent that poor prognasis is considered a core characteristic of the schizophrenia concept, these variables, by their established predictive validity, are obviously related to this concept. Because of this relationship Affective Flattening, Social Isolation and Premorbid Asociality are also referred to as 'schizophrenia wariables'. Some degree of intercorrelation between these three characteristics can be expected, especialiy between Premorbid Asociality and Social Isolation, because both seem to denote esentially the same behavioral characteristics of the individual, although separated in time. The relevance of these diagnostic variables to the non-schizophrenic diagnostic categories is less clear. The postulated relationship between schizophrenia and achizotypal personality disorder suggests that this latter category will feature relatively high scores on these variables, al though probably less high than the schizophrenic patients. The distinction between schizophrenic and non-schizophrenic types of psychosis is far from clear, and is arbitrarily made here by the DSM-III criteria. It is aupposed, however, that the schizophrenic and schizotypic 
categories will shou higher scores an these variables than the nonschizophrenic psychotic category, as distinguished in this investigation. Affective Flattening and Social Isolation are both included in the DSM-III sets of criteria for the diagnoses of schizophrenia and of schizotypal personality disarder, but they are neither sufficient nor necessary for these diagnoses. In addition they are scored on an all-or-nothing basis in DSM-III, criteria not specified, while the "schizophrenia variables' provide a dimensional representation, because they are scored on severity scales. There is thus an essential difference with the categorical construct of the diagnosis of schizophrenia according to DSM-III. Subjects from all diagnostic groups are given scores an these 'schizophrenia variables'.

The fourth major diagnostic approach in this investigation is Psychoticism, defined according to the Eysenck Personality Questionnaire. This is a dimensional personality description, essentially different from 'traditional' diagnostic approaches. Nevertheless, Eysenck has hypothesized relations with psychiatric diagnosis, in particular with the diagnosis of psychotic disorder. It follows that both psychotic groups in this investigation should have the highest Paychoticism scores. Although not predicted by Eysenck, it seems reasonable to suppose that the schizotypal patients, because of their borderline character, will occupy an intermediate position between psychotic patients and meurotic patients, and that normals will have the loweat Psychoticism scores. There is, in short, reason to assume a gradual decline of Psychoticism scores across the range of psychiatric diagnoses, both psychotic groups perhaps occupying a comparable high rank, although the chronicity criterion of schizophrenia provides arguments ta maintain the schizophrenics on the highest rank.

The fifth major diagnostic approach is represented by the set of attentional variables. This approach is entirely different from the other ones, and is supposed to indicate controlled information processing deficits in an objective way. These measures are therefore considered to be more reliable indicators of psychiatric dieturbance 
than traditional diagnostic descriptions, as represented by the other, non-attentiona1, variables in this investigation, which are brought together in the first four major diagnostic approaches. Poor quality of Eye Tracking, slow Mean Reaction Time, Reaction Time Redundancy Deficit, poor performance on the Continuous Pierformance Test, low amplitude of the Contingent Negative Variation, and Post Imperative Negative Variation have all shown in previous research an assocation with a diagnosis of psychosia, more particularly a diagnosis of schizophrenia (discussion in the next chapter). The distinction in this study between groups of schizophrenics, non-schizophrenic psychotics and schizotypics allows the analysis of the relevance of deviant attentional performance to the schizophrenia concept in more detail than when only schizophrenic patients would have been included. At least, to the schizophrenia concept and related concepts inherent in DSM-III, and of course to the extent that the selected attentional variables may be considered reliable indicators of attentional or controlled processing deficits. It is assumed here that poor attentional performance is not limited to schizophrenic psychotic patients, but is also present, although to a lesser degree, in less disturbed psychiatric patients. In other words, an association with degree of disturbance, or severity of psychiatric diagnosis, rather than with particular diagnostic categories, is assumed.

From the assumptions discussed in the preceding section the following hypotheses are derived.

Concerning the intercorrelations within the attentional set of variables:

I. The electrophysiological and performance measures of attention in this investigation - Smooth Pursuit Eye Movements (Total and Best Cycle), Reaction Time (Mean and Redundancy Deficit), Continuous Performence Test (Simple and Complex Versions, Omission scores), and Contingent Negative Variation ( $\mathrm{Cz}$ and $\mathrm{Fz}$ derivations, Early and Late Components, and PINV) - are significantly positively intercorrelated. 
Concerning the relations between psychiatric diagnosis and diagnostic varlables:

II. The diagnostic measures Affective Flattening, Social Isolation, Premorbid Asociality, and psychiatric diagnosis, rank ordered: normal - neurosis - non-schizophrenic psychosis - schizotypal personality disorder - schizophrenia, are significantly positively intercorrelated.

III. Psychoticism and severity of psychiatric diagnosis, diagnases rank ordered: normal - neurosis - schizotypal personality disorder - mon-schizophrenic psychosis - schizophrenia, are significantly positively correlated.

Concerning the relations between psychiatric diagnosis and attentional variables $(=$ CENTRAL HYPOTHESIS):

IV. Degree of irregularity of Smooth Pursuit Eye Movements, Duration of Mean Reaction Time, degree of Reactian Time Redundancy Deficit, percentage of amissions on the Continuous Performance Test, degree of depression of the Contingent Negative Variation, and level of Post Imperative Negative Variation are significantly positively correlated with severity of psychiatric diagnosis, diagnoses rank ordered: normal - neurosis - schizotypal personality disorder - non-schizophrenic psychosis schizophrenia.

\section{Explorative analyses.}

Explorative analyses offer the opportunity to study the relovance of attentional dysfunctioning, as operationalized by the selected tasks, to specific diagnostic categories, especially schizophrenie and related disorders, instead of to the severity of psychiatric diagnosis, and to compare the attentional variables with the other - "diagnostic" - independent variables with respect to indication of severity of diagnosis as well as discrimination between diagnostic 
categories. Univariate as well as multivariate statistical analyses are therefore applied to the data, in particular analysis of variance, multiple regression analysis and discriminant analysis. In addition a clustering procedure is applied, to compare clusters thus generated with traditional (DSM-III) categorical classification.

The explorative part of this investigation concerns some additional diagnostic variables, namely the other EPQ scales - Extraversion, Neuroticiom, Lie - which do not seem to be primarily relevant to psychotic dysfunctioning and are therefore not included in the hypotheses. CPT Commission scores participate also in the explorative analyses. Although these variables have been shoun to be associated with psychosis in earlier studies, it is not clear that they should represent attentional dysfunctioning. 
INSTRUMENTS AND METHODS

\subsection{DIAGNOSTIC DESCRIPTIONS.}

\subsubsection{Subject classification: psychiatric diagnosis.}

In the present investigation the DSM-III criteria (APA, 1980) are used for diagnostic classification of the subjects in the patient groups. These criteria have partly been developed from the Research Diagnostic Criteria and prabably represent the most solid diagnostic system currently available, although its arbitrariness should be acknovledged (e.g., Fenton, Mosher and Matthews, 1981). An additional. argument in favor of these criteria is their expected yidespread use in the near future. Presumably, the term schizöphrenia will soon and increasingly be equated with the definition inherent in the DSM-III criteria, especially in the USA, but probably also in other countries. Comparability and interchangebility of research findüngs are heavily dependent on similarity of diagnostic concepts, a fact which is still too often ignared. Widely accepted diagnostic criteria promote an easy exchange of research findings, which is an additional reason to prefer the DSM-III criteria.

The interrater reliability of DSM-III has been shown to be generally higher than previously achleved. The averall Kappa coefficient of agreement for major psychiatric diagnoses of 281 adult patients was .78 for joint interviews and .66 for separate interviews $(.82$ for the 
diagnosis of schizophrenis in both conditions). For personality disorders these coefficients were .61 and .54 (Spitzer, Forman and Nee, 1979). The relatively high reliability of DSM-III is clearly the result of the use of diagnostic criteria. Ward, Beck, Mendelson, et al. (1962) have shoun that the major source of diagnostic unreliability has always been the use of inadequate nosological methodology. Unly a small part of disagreement was shoun to result from differences in interviewing technique.

\section{Schizophrenia and other psychotic disorders.}

In the case of schizophrenia the differences between DSM-III and the Research Diagnostic Criteria are not very great (for DSM-III criteria of schizophrenia: see Appendix-1). Some changes have been made in the characteristic symptoms and the presence of one of these symptoms is considered sufficient. An important change is the requirement that signs if the illness be present continuously for at least 6 months or have at some time during the person's life been present for such a period. Signs of the illness may be one or more of the characteristic schizophrenic symptoms or two or more prodramal or residual symptoms, but characteristic symptoms must have been present during an active phase. Excluding patients whose duration of the illness has been less than 6 months implies choosing for the 'poor prognosis' concept of schizophrenia, as a long duration of symptoms ranks among the most powerful indicators of a poor prognosis. The DSM-III criteria do not require an early age of onset, but the diagnosis of schizophrenia is limited to individuals whose first episode of illness occurred before the age of 45 (Spitzer, Andreasen and Endicott, 1978).

Psychotic patients without evidence of arganic disturbance are in this investigation categorized as schizophrenic or mon-schizophrenic. Included in the non-schizophrenic psychotic group are patients with other types of functional psychoses with signs of the illness lasting at least one week. The clinical picture in these cases involves at least one of the following: delusions, hallucinations, or gross disorganization of speech or behavior. The following DSM-III 
categories are included in this group: paranoid disorders, schizo-affective disorder, schizophreniform disorder, brief reactive psychosis and atypical psychosis. Excluded are psychotic reactions lasting no more than one week, and the affective disorders. Details on this diagnostic category are in Appendix-1.

Schizo-affective psychoses are placed here under the heading 'nonschizophrenic psychoses'. There has been a great deal of debate as to the meaning and the validity of the concept of schizo-rffective psychosis (Procci, 1976). Controversy exist as to whether this condition is to be considered as a subgroup of schizophrenia, as more closely related to the affective disorders or as a distinct entity. Presumably the schizo-affective disorders are part of a continum between "pure affective disorder' and 'pure schizophrenia' . Tsuang and Dempsey (1979) concluded that with respect to prognosis the schizoaffective disorders seem to fall between schizophrenia (slightly modified St. Louis criteria) and the affective disorders.

Schizotypel personality disorder and other mon-psychotic (neuratic) disorders.

The group of non-psychotic non-organically disturbed psychiatric patients participating in this investigation is also dichatomised, like the psychotic group. These subjects are categorized according ta the presence of 4 or more (of a total of B) schizotypal features, ss first defined by Spitzer, Endicott and Gibbon (1979) and later included in DSM-III. Excluded from these categaries are subjects who at any time in their life had a psychotic episode. One graup of nonpsychotic patients fulfils the DSM-III criteria of schizatypal personality disorder (criteria are in Appendix-1), the other group consists of patients meeting various diagnoses from the following DSM-III categories: Dysthymic Disorder, Anxiety Disorders, Somatoform Disorders and Dissociative Disorders. Although DSM-III does not make use of the term 'neurotic', the categories just mentioned are about equivalent with this global category. It should be stressed that the schizotypal subjects do also meet the criteria for one or more of 
these nonpsychotic diagnostic categories.

Spitzer, Endicott and Gibbon (1979), studying the clinical utility of the schizotypal item set, found very good sensitivity (the proportion of the total number of cases that are correctly identified, within a group of patients given a clinical diagnosis of borderline schizophrenia) and specificity (the proportion of the total number of noncases that are correctly identified) using a cutoff of at least 4 items (sensitivity: $80 \%$; specificity: $89 \%$ ). This cutoff-point was therefore made criterion for the diagnosis of Schizotypal Personality Disorder in DSM-III.

Normal controls.

The fifth group of subjects participating in the present investigation are normal controls. They have not and never had a psychiatric disturbance to such a degree that they sought professional help for it. 
5.1.2. Dimensional descriptions, related to the schizophrenia concept.

In this investigation, apart from diagnostic classification, a small number of variables with established predictive implications (predicting a poor prognosis - WHO, 1979) is introduced. The selected variables - Affective Flattening, Social Isolation and Premorbid Ascoiality - characterize the 'core' schizophrenic syndrome, the poor prognosis schizophrenia, the schizophrenia concept according to the European - Kraepelinian - tradition. These variables are scored here on severity scales.

\section{Affective Flattening.}

The first of these variables is the phenomenon of Affective Flattening (used here as synonymous with emotional blunting, although in practice there may sometimes be subtle semantic differences, that are difficult to evaluate however, but are probably related to differences in degree of restriction of affective response). The reliability of the determination of the presence or absence of this feature has always been a problem. This raliability has, for instance, been found unacceptably low by Luria and Mchlugh (1974), using the Present State Examination. Recently, however, rating scales have been developed to evaluate flattening of affect.

Abrams and Taylor (1978) reasoned that the reliability of the assessment of affective flattening might be increased by using a rating scale in which a global impression is broken down in a number of separate ratings of the component parts. They therefore reviewed the literature for clinical descriptions of affective flattening, starting, as they report, with Pinel and ending with Kraepelin. Their scale thus gathered is composed of 16 items, organized under three headings relating to affect, behavior and thought content. The authors provide evidence indicating a good reliability of the scale and generally good reliability of the individual items. Patients diagnosed 
as achizophrenics had much higher mean scores than patients diagnosed as manics. The scale discriminated clinically diagnosed schizophrenic patients from patients with affective disorders and predicted (poor) short-term treatment response.

Andreasen (1979) developed a rating scale for affective flattening, based on an empirical approach rather than the historical one of Abrams and Taylor (1978). Her scale confirms the finding that affective flattening can be defined reliably. Because the scale of Abrams and Taylor includes a broader behavioral spectrum it is preferred in the present investigation (see Appendix-2).

\section{Social Isolation.}

The second variable with established predictive validity that is included in this investigation is Social Isolation. This feature is already part of the Rating Scale for Emotional Blunting just mentioned. It is judged separately, however, using the 'Social Functioning" section of the Schedule for Affective Disorders and Schizophrenia (Spitzer and Endicott, 1978) (see Appendix-3). This short rating scale does not limit itself to the present situation, but covers the period of the past 5 years and refers to the best level of social relations that lasted at least several months.

\section{Premorbid Asociality.}

Premorbid social functioning is the third of the added diagnostic variables. Past histary of psychosexual adjustment, a significant predictor from the International Pilot Study of Schizaphrenia, is included in the Premorbid Asocial Adjustment Scale, latest edition, of Gittelman-Klein (in: Kokes, Strauss and Klorman, 1977). This scale is included in Appendix-4. This premorbid function scale has been devised specifically to assess premarbid asocial adjustment in schizophrenics. The scale limits itself to 'aspects of a shut-in, schizoid, withdraun, asocial premorbid personality during preadolescence and adolescence" (Gittelman-Klein and Klein, 1969). 
Ratings are made for the periods of preadolescence and adalescence on subscales for isolation, peer relationships and interests. A seventh subscale measures interpersonal heterosexual involvenent between the age of 16 and 20 . Dverall score is usually computed by averaging across all scored items if at least three of the seven subscales have been rated. Ratings are made only for behavior anteceding the first manifestation of psychotic symptoms. Reliability of this - since its first publication slightly modified - rating scale has appeared good. Concerning validity, scores appeared significantly correlated with measures of posthospital adjustment including psychiatric status, occupational adjustment and interpersonal functioning (Gittelman-Klein and Klein, 1969). For the present investigation choice fell upon the Gittelman-Klein scale, because unlike other premorbid functioning scales (for an overview: Kokes, Strauss and Klorman, 1977) it limits itself to one dimension of premorbid functioning: social, interpersonal functioning. Premorbid functioning is a multidimensional concept (Strauss and Carpenter, 1977; WHO, 1979), and putting different dimensions together in one measure confuses the issue. Besides, marital status and employment function, which are major parts of other scales, seem inappropriate, because current social and cultural developments make these items unreliable. 


\subsubsection{Dimensional personality description: psychoticism.}

In this investigation the dimensional approach in description of personality is represented by the Eysenck Personality Questionnaire (EPQ), Adult Version (Eysenck and Eysenck, 1975) (see Appendix-5). A Dutch translation of this personality questionnaire is introduced in this investigation primarily for its Psychoticism (P) scale. It also contains scales for Extraversion (E) and for Neuroticism (N) and a Lie (L) scale.

Psychaticism is a personality dimension, postulated by Eysenck to be a measure of the predispoeition to psychosis. He stresses that the concept of peychoticiom makes sense only within a dimensional system of personality description. The EPQ is the first 'official' questionnaire including a P-scale. Earlier prototypes were selected by Eysenck over a series of studies on their content validity, until a factor emerged that was largely independent of extraversion and neuraticiem. The P-scale has undergone many changes in the course of these studies. Eysenck has posited a genetic basis of the $P$ dimension. In addition it should run through the normal range of personality manifestations and it should be measurable by reference to behavioral traits rather than psychiatric symptoms. In his opinion these positions are now well supported by research findings (Eysenck and Eysenck, 1976). There will be no digression on these topics here. What concerns us most is the question of validity. Validity of the P-scale was, according to Eysenck and Eysenck (1977) their primary concern and considered more important than reliability.

External validation of psychoticism is not a simple task. Despite the criticism of the categorical ('medical') model of diagnosis, the concluaton of a dimensional approach to diagnosis being clinically meaningful implicitly assumes the validity of a clinical categorical model and to this extent any inferences drawn may be regarded circular. The methods of validation chosen by the Eysenck's for their P-scale are the following (Eysenck and Eysenck, 1976). The first. 
method is the testing of criterion groups. Certain groups, in particular psychotics, should have elevated $P$ scores. The second method of demonstrating validity consists in correlating $P$ scores with scores on objective and laboratory teats. High $P$ scorers should score in such a manner, as compared with low p scorers, as do psychotics as compared with normals. Eysenck (1977) later added a third method of validation: Within a psychotic population those more seriously ill should have higher $P$ scores than those less seriously i11.

What kind of personality does the P-scale describe? According to Eysenck and Eysenck (1976) the psychological meaning of the scale suggests that the $P$ personality is 'solltary, troublesome, cruel, lacking in feeling, lacking in empathy, hostile to others, sensation seeking, and liking odd and unusual things'. Few items refer to behaviors commonly recognized as characterizing psychotics. The scale is heavily weighted on impulsive, agressive, and socially deviant traits. It is not surprizing, therefore, that in the standardization data (Eysenck and Eysenck, 1975) psychotics did not produce significantly different scores from prisoners, drug addicts and alcoholics. However, the same lack of differences was found with persons with personality disorders and with sexual problems. Male art students even had the highest $P$ scores of all.

Eysenck and Eysenck (1976) try to explain the failure of the P-scals to discriminate psychotics from criminals by referring to a genetic relationship between psychosis and psychopathy. However, this relationship is open to criticism (e.g., Gottesman and Shields, 1976) and, in addition, imprisomment, criminality and psychopathy do not: simply qualify the same persone. Concerning the other high $P$ scoring groups explanations are lacking. Eysenck (1977) further argure, that prychotics tend to have high Lie scores, which lowers their $P$ acorea below what they would otherwise be, whereas the other groups do not have such elevated $L$ scores. But in psychotics with low $L$ scores the mean $P$ score is still exceeded by or nearly equivalent to the scores of several other groups. Even for Eysenck these facto are not easy to 
explain. However, he has emphasised that the purpose of the P-scale has never been to aid in the diagnosis of psychotics. In a reply to criticisms of the P-scale Eysenck and Eysenck (1977) even state that they do not regard discrimination between psychiatric graups as constituting a useful criterion, because of the unreliability and the arbitrariness of diagnoses, but that they are interested in the degree of overlap between the dimensional and the categorical systems. It seems they are contradicting here their own choice of validation method. According to Eysenck and Eysenck (1976) other methods of validation have resulted in much evidence of support for their identification of $P$ as a measure of the predisposition to psychosis, but the evidence they provide is rather limited. The participation of the Psychaticism scale in the present investigation primarily represents an example of Eysenck's first method of validation, but his second method is also represented.

Eysenck and Eysenck (1976) have among others tried to find support for their claim of $P$ as related to the vulnerability to psychosis in a relatively small number of mainly unpublished studies on attentional functioning. These studies usually included an earlier version of the $P$ scale. According to their second validation method, high scores on the $P$ scale are hypothesised to correlate with deviant performance on attention tasks in the same manner psychotic patients do. Among the relevant studies are the following. Eysenck and Eysenck (1976) refer to a study of Clements (1970, unpublished), who found a correlation (significant?) of high $P$ score with slowness on a reaction time task. Details are not given, and instead of the EPQ the earlier version named PI was used. According to the Eysenck's, two other investigators (Hendrickson, 1972 and Thompson, 1973, both unpublished, cited in Eysenck and Eysenck, 1976) recorded shorter reaction times in high $P$ scorers, rather than the expected longer reaction times. However, this was found only in trials without warning signal. When warning signals were given, no relationship with $P$ was found. It seemed that high $P$ scorers are hampered by the addition of a warning signal, as compared to low $P$ scorers, and this could be explained in terms of failure to preserve set. Both investigators also used an earlier version of the 
$P$ scale. Stroh (1969, unpublished, cited in Eysenck and Eysenck, 1976) tested normal subjects on a one-hour visual vigilance task. P scores proved to be significantly correlated with the signal detection theory measure of sensary threshold. In this investigation the PEN inventory was used. Hinton (1976) hypothesized that, if the P scale (PEN inventory) measures an aspect of psychotic behavior rolating to attention difficulty, then $P$ score should be positively correlated with an electromyographic measure of muscle tension (M. Trapezius) as an index of attentional effort on perceptual discrimination tasks. He found significant correlations in a student group, whereas correlations of this index with $N$ and $E$ were insignificant. He repeated this experiment with male patients in a prison for the criminally insane, but now a significant correlation in the opposite direction was found: (Hinton, cited in Eysenck and Eysenck, 1976). The explanation was sought in their higher $L$ scores, indicating their. generally defensive attitude especially to $P$ scale items. In this sample $L$ score was positively correlated with muscle tension.

Somewhat more complicated relationships between $P$ and psychophysiological measures were studied by Claridge and Chappa (1973). High $P$ scorers (normal subjects) were found by these investigators to show the same U-shaped relationship between two-flash threshold and skin conductance level as did normal subjects given LSD (purported to represent a drug model of psychosis) (Claridge and Hume, 1966; Claridge, 1972) and schizophrenic patjents (Venables, 1963). Normally, an inverted U-function is found for this relationship. In the first experiment the difference between high $P$ scorers and high $N$ scorers vas obvious: they showed significant, but opposite correlations. Low $N$ scorers behaved like high $P$ scorers, however, which was post hoc explained by the authors by suggesting "that individuals reporting low neuroticism scores cauld be regarded as having the lack of anxiety and emotional responsiveness appropriate to certain forms of psychaticiam which are not tapped by Eysenck' $\theta P$ scale'. The fact that in this study use was made of the PEN inventory has led to some discussian concerning the relevance of these findings to the EPG-P scale (Block, 1977; Block, 1978; Claridge and Birchall, 
The studies mentioned here seem to suggest some relationship between $P$ score and attentional dysfunctioning, although the evidence is rather ambiguous. As comparable defects of attention have been found to correlate with psychosis in studies of psychiatric patients, these findings may to some degree be explained as supporting Eysenck's proposition that $P$ measures the predisposition to develop a psychotic disorder. It should be remembered, however, that these studies made use of earlier versions of the $P$ scale, which has been changed in the course of its development. This means that the relevance of the rather meager results of these atudies for the validity of the current $P$ scale is uncertain. There is one report of relations between EPQPsychoticism scores and a sorting task, which concerned people"s ability to attend to component features in the set of complex stimuli (Griffith, Frith and Eysenck, 1980). Of the EPQ-scales, only Psychaticism appeered to relate to performance on the sorting test, but this relationship was rather weak. Further studies of relationships between attentional-information processing deficits and Psychoticism (EPQ) seem warranted and necessary in order to fully appreciate the significance of this personality measure.

Something should be said about inter-cultural comparison studies of the $P$ scale. The basic personality dimension Psychoticism is hypothesized to represent should, of course, not be limited to certain cultures, for instance the Europeen-American culture in which the questionnaire was developed. Eysenck and Eysenck (1976) refer to studies in Nigeria, Japan, Australia, India, South Africa, Iran, Hawail, Ireland, Israel and Czechoslovakia. They conclude that the results of these studies seem to indicate that the scales constructed in Britain apply pretty much in the same way in a variety of other cauntries. The fact remains that the $P$ scale has undergone changes, and thet the EPQ version differs from the earlier PEN version, usually used in these studies. 
Janssen (1973) has demonstrated the same factor structure in a Dutch translation of the PEN inventory as Eysenck found in the English version, so psychoticism proved to be a factor of the Dutch version of the PEN inventory. Unfortunately factor analytic investigation of a Dutch translation of the EPQ has yielded various $P$ factors that did not prove to be very similar, so that the consistency of the Dutch EPQ-P scale has still to be considered questionnable (Van Kampen, 1976). This finding weakens the degree to which Dutch $P$ scores can be considered to relate to the basic personality dimension Psychoticism is hypothesized to be. It vas nevertheless considered interesting to include this personality measure in this investigation. Van Kampen did find consistent $E$ and $N$ scales in the Dutch EPQ translation, so that no problems arise as to the interpretation of these variables in this investigation. 


\subsection{ATTENTIONAL TASKS.}

\subsubsection{Smooth Pursuit Eye Movements.}

The quality of Smooth Pursuit Eye Movements (SPEM), or eye tracking, has been recognized as a measure of (more or less involuntary) attentional functioning, although it is obvious that especially late atages of information processing (motor function) have an important role in this function.

In 1908 Diefendorf and Dodge were the first investigators who described inadequate smooth pursuit movements of the eyes in psychiatric patients with diagnoses of mania, depression, dementia praecox, dementia paralytica, and others. Dementia praecox patients perfarmed worst of all. Their eye tracking was characterized by step-like movements, in the opinion of the investigators not explainable as defects of attention, because, among other things, reasonable attention and effort at pursuit were considered to be clearly indicated in the number and character of the shart corrective (saccadic) movements. Replication of these findings was attempted by Couch and Fox in 1934. Steplike interruptions of the pursuit eye movements appeared in the records of 14 of the 43 patients with diagnoses of dementia praecox. Of a total of 117 psychiatric patients 27 displayed this 'stepping' peculiarity. Although Diefendorf and Dodge believed this phenomenon to be characteristic of dementia praecox, it appeared not confined to this type of disorder, and 3 dementia praecox patients even revealed normal pursuit movements.

Recently, beginning with the work of Holzman, Proctor and Hughes (1973), the study of smooth pursuit eye movements in psychiatric patients has received new interest. Although the relationship of this measure of central nervous system function to the concept of attention seems complicated, the rejection by Diefendorf and Dodge (1908) of attentional deficits as relevant to this phenomenon seems 
unjustified. Eye tracking measures reflect the motor output pattern of a quite complicated information processing sequence with multiple feedback mechanisms and excitatory as well as inhibitory controls. There is no decisive difference in this respect with other performance measures of attentional functioning, but the balance between controlled and automatic pracessing seems more in favor of the latter than in many other attentional measures.

Investigations of these eye movements typically require subjects to visually track a moving object, an oscillating pendulum or a horizontally moving spot on an oscilloscope or television screen, the velocity of which is varied sinusoidally. Eye movements are usually recorded electro-oculographically. An adequate performance of the subject results in an electro-oculogram consisting of a continuous series of reasonably smooth sine waves. There are other methods for monitoring eye movements, for instance the infrared reflection technique, which is based on the differential reflectivity of the iris and sclera to infrared illumination. Lindsey, Holzman, Haberman and Yasillo (1978) and Iacono and Lykken (1981) have compared both methods in psychiatric patients and normal controls. Although the infrared recorded traces were generally somewhat smoother than the electrooculogram, there was excellent correspondence between tracings for each subject. This is an important finding, because several investigators have used this technique instead of electro-oculagraphy. Excursions of the fixation object, pendulum or moving spot, typically correspond to 20 degrees of visual angle. The sinusoidelly modulated movement has a frequency of 0,4 or $0,5 \mathrm{~Hz}$. This implies that maximum eye velocity during perfect tracking is about 30 degrees/aec. Advantages of the oscilloscope method are the constancy of the excursions and the limitation of the movement to the horizontal plane. There have been experiments with other movement patterns: a back and forth horizontal movement at a constant speed (Shagass, Amadeo and Overton, 1974; Kuechenmeister, Linton, Mueller and White, 1977), a circular movement (Shagass, Amadeo and Overton, 1974) and sinusoidally varied vertical and diagonal movements (Cegalis and Sweeney, 1979; Lipton, Levin and Holzman, 1980). These alternatives have no 
advantages. Differences between the smoath pursuit patterns are not significant.

Before considering the results until now of studies with modern techniques of smooth pursuit eye movements in psychiatric patients, especially schizophrenics, some information on the oculomotor system is presented. The major function of the oculomotor system is probably to stabilize retinal images, because the retinal image is displaced by everything we do (Steinman, 1975). The analogy with the general attentional functions of selecting and focussing is clear. In the human eye tracking syatem two major modes of tracking can be recognized: rapid saccadic eye movements and smooth pursuit eye movements. Saccadic movements are sudden shifts of gaze between two points of gaze equally distant from the observer. These movements are extremely fast and act to bring the image of the object of interest on the fovea centralis of the retina. The smooth pursuit system stabilizes the retinal image and keeps it on the fovea. This system responds only to target velocity and matches the angular velocity of the eye to the velocity of the target (Rashbass, 1961). This matching occurs up to eye velocities of approximately 30 degrees/sec., though on occasion the maximum velocity of the eye in smooth pursuit may be three to five times as large (Young, 1971; Alpern, 1972). It is probable that, at the other side of the velocity spectrum, the pursuit system tracks velocities down to, and including, zero (Robinson, 1971). The 'hold' system thus seeme to be part of the pursuit aystem. Besides target velooity, the pursuit movement system uses many other sources of information: visual, vestibular, proprioceptive and fferent command associated with body movements (Young, 1971).

The anatomical details of the pursuit eye movement system are incompletely known, but relevant structures seem to be extensive. It seems probable that both hemispheres, especially the occipital and parietal lobes, participate to produce smooth pursuit movements in all directions (Hoyt and Frisén, 1975). Pursuit eye mavements seem to originate cortically in the occipital-parietal visual association 
areas and to be ultimately regulated, at least the eye movements in the horizontal plane, in the pontine paramedian reticular formation (Hoyt and Daroff, 1971).

Modern research on smooth pursuit eye tracking in psychiatric patients began with studies of Holzman and coldaborators (Holzman, Proctor and Hughes, 1973; Holzman, Proctor, Levy, et al., 1974). They found a striking association between deviant smooth pursuit eye movements and schizophrenia. In their first study they found significant differences between schizophrenic groups (paranoid, nonparanoid and schizoaffective) and groups of psychiatric patients with manic-depressive and nompsychotic disorders and normal controls. Their second report concerned larger groups of patients: 40 'recent' schizophrenics with periods of hospitalization up to six months, 29 chronic schizophrenics, 6 schizo-affective patients, 9 other psychotics, 19 nonpsychotic psychiatric patients and 72 nonpatient control subjects. 52\% of the "recent" schizophrenics, $86 \%$ of the chronic schizophrenics and 50\% of the schizo-affective patients showed deviant eye tracking. This contrasted with the percentages of the other groups: $22 \%$ of the other psychotics, $21 \%$ of the nonpsychotic patients and $8 \%$ of the normal controls. The impaiment in smooth pursuit eye tracking was, according to Holzman, Proctor and Hughes (1973), characterized by fast, horizontal saccadic-like movements superimposed on the pursuit, and by other kinds of deviations from the sinusaidal pattern. Weak points in these first reports are the lack of diagnostic criteria, and the limitations of the scoring techniques, which will be discussed later. Another limitation in these first studies was the wee of a pendulum as the visual stimulus, because it lacks precision arid flexibility. Although later atudies have improved on many pointa, diagnosis remained a point of weakness.

Dther investigators have confirmed the finding of Holzman et al. in showing significantly poorer smooth pursuit eye tracking in schizophrenic patients than in normals. Shagass, Amadeo and Overton (1974) found significantly impaired eye tracking in schizophrenice but also in patients with affective psychoses. There vere no significant 
differences between nonpsychotic psychiatric patients and monpatient controls. Diagnostic criteria were not mentioned by these authors. Holzman and Levy (1977) later reported that they too found large numbers of non-schizophrenic patients to show deviant pursuit eye movements, but they did not offer details on this observation. These findings auggest that deviant eye tracking is not specific to schizophrenia, but a phenomenon associated with functional psychosis in general. Later studies have hardly compared schizophrenic patients with non-schizophrenic psychatic patients. May (1979) included two patients with a (non-functional) amphetamine psychosis. The eye tracking patterns of these patients did not differ significantly from those of normal subjects. Pursuit eye tracking of schizophrenic patients in this study was significantly inferior to that of control subjects. Lipton, Levin and Holzman (1980) and Levin, Lipton and Holzman (1981) tested schizophrenic and manic-depressive patients meeting the $5 t$. Louis diagnostic criteria. Although tracking quality was significantly poorer in both patient groups than in normals, there were no significant differences between the two patient groups.

The significantly poorer quality of eye tracking in chronic and acute schizophrenics than in normal controls was confirmed by Brezinova and Kendell (1977); Acker and Toone (1978); Cegalis and Sweeney (1979); and Latham, Holzman, Manschreck and Tole (1981). Cegalis and Sweeney found in addition generally insignificant differences between acute and chronic schizophrenics. Kuechenmeister, Linton, Mueller and White (1977); Pass, Salzman, Klorman, et al. (1978) and Mialet and Pichat (1981) also found significantly impaired eye tracking in young schizophrenics, compared with normal controls and with nonpsychotic patients of the same age. Most of these studies are, like the earlier ones, characterized by a lack of clear and reliable diagnostic criteria.

Holzman, Proctor, Levy, et al. (1974) reported a sharper association between schizophrenia and deviant pursuit eye movements when the schizophrenic group was defined as such on the basis of thought disorder as expressed in the WAIS and the Rorschach test. Distinction 
according to paranoid status, number of previous hosplartutions at prenorbid functioning did not result in differences on pursult eye tracking measures of (sub)acute schizophrenics. There also seens to be no correlation between deviant smooth pursuit eye movements and severity af psychosis (Holzman, 1975 ). However, of special interest are correlations found between quality of eye tracking and personality trats called psychoticism and absarption as measured by the Differential Personality questionnaire (Tellegen and Atkinson, 1974; Lykken, Tellegen and Derubeis, 1978; Tellegen, 1978; Iacono and Lykken, 1979-a), and with psychoticiem as meesured by the Eysenck Personality questionmaire (1acono, 1978).

At the moment, the question of diagnostic specificity has not been resolved, probably partly as the result of dianostic unreliability as we11 as heterogeneity. In one study schizophrenic autpatients (to what degree semited?) even performed, although lese bcurately, not significantly different from normal controla, and signifieantly better than psychietric inpatients with varying diagnoses (Pivik, 1979-a: b). Newertheless the hypothesis that dewiant smooth pursuit aye movements represent a trait characteristic and to some degree a genetic marker of schizoptrenit has received considerable support. Salzman, Klein and Strakss (1970) studied women with a history of psychiatric hospitalization, but at the time of the study functioning in the commuty and at least in partial remission. part of the group had received diagnosis of schizophrenia (meinly schiza-affective psychosis), part had been hospitalized for another serious poychiatric disorder. In this study only the degree of paychosis at the time of the subject"s prior hospitalization was significantly related to the wye tracking performance, but the achizophrenic group was probably rather heterogeneous. There vas no significant relation with ourent level of functioning or diagnosis. Comparable reaults urere reported by Iacono, Tuason and Johnson (1981) in patients diagnosed schizophrenic according to the Research Diagnostic Criteria, but currently in remission. In a brief report Klein, Salzman, Jones and Ritzler (1976) reported on children of the women of their 1978 report. Dividing these children into groups according to schizophrenic versus 
non-schizophrenic diagnoses in mothers did not result in significant differences on eye tracking scores. There was no comparison with children of normal control mothers. Correlations between eye tracking and global adjustment or anxiety scores were not significant. As the deaign of this study was inadequate, the question of deviant eye tracking in wulnerable or high-risk children has not yet been readived.

The stability of eye tracking performance has generally appeared to be good. Holzman, Practor and Hughes (1973), Shagass, Amadeo and Overton (1974), Iacono and Lykken (1979-b; $c ; 1981)$ found generally good retest reljabilities in nompatients, computed across periods ranging from one yeek to two years. Among chronic psychiatric patients, Holzman, Proctor, Levy, et al. (1974) found no significant change in eye tracking performance at retesting, after they had been removed from medication for ten days. Shagass, Amadeo and Overton (1974) retested psychiatric patients, first tested free of drugs, after intervals up to more than a month, while they vere receiving various kinds of drugs. Test-retest reliabilities were significant. These findings suggest that performance on smooth pursuit eye tracking tasks characterizes a stable trait.

Evidence on the genetic contribution to this trait has come from several studies. Holzman, Proctor, Levy et al. (1974) found deviant eye tracking in $44 \%$ of schizophrenic patients" first degree relatives who were not themselves olinically schizophrenic, compared to $10 \%$ of relatives of non-schizophrenic psychiatric patients and 8 \% of normal control subjects. The percentages of deviant eye tracking in recent and chronic schizophrenics in the same investigation were $52 \%$ and $86 \%$ respectively. In additional data on first degree relatives the asgociation between deviant ye tracking and schizophrenia in the family continued to show itself (Holzman and Levy, 1977). Kuechenmeister, Linton, Mueller and White (1977) compared parents of schizophrenics with age-matched controls and found significant differences in eye tracking scores, against the former. 
The hypothesis that deviant pursuit movements represent genetic indicator of schizophrenia was directly tested in a study of monozygotic and dizygotic twins discordant for schizophrenia (Holzman, Kringlen, Levy, et al, 1977; 1978). The data shawed a considerably higher concordance for poor eye tracking thain for schizophrenia. The concordance rates were in the correct direction for genetic effects, but because of the small numbers firm conclusions were not justified. Environmental causes could not be ruled out in this study, which was another reason for cautiousness in interpreting the results. In addition, age was suspected to be negatively related with quality of eye tracking and this factor could have interfered too in the twin study, because the average age was 55 years. However, Holzman, Kringlen, Levy and Haberman (1980) later repeated theúr study of eye tracking in twins with younger pairs, and replicated their earlier findings. Iacono and Lykken (1979-a; b) studied non-patient, young adult monozygotic tuins and found that these twins performed similarly on a number of eye tracking tasks, so the similarity among twins seems not the result of posisible genetic concordance for neurophysiological concomitants of maturation. The correlation of eye tracking measures between monozygotic twins was . 77 in the Holzman et al. (1980) study, and .69 in the Iacono and Lykken study. In conclusion, deviant smooth pursuit eye movements seem to represent a reasanably stable trait, to a considerable extent genetically controlled and rather characteristic for schizophrenic psychotic patients and, to a lesser degree, other psychotic patients.

As has already been mentioned, age may have some influence on eye tracking performance. For instance, Shagass, Amadeo and Overton (1974); Kuechenmeister, Linton, Mueller and White (1977) and Iacono, Tuason and Johnson (1981) concluded that the quality of eye tracking generally seems to decrease uith age, but Pivik (1979-a) and Iacono and Lykken (1979-a) found no relationship between age and performance. The influence of sex seems negligible. Kuechenmeister et a1. (1977) found a tendency for men to perform better than women, but Shagass et al. (1974), Pivik (1979-a), Iacono and Lykken (1979-a) and Mialet and Pichot (1981) found no significant sex differences. Target velocity 
does not seem to be relevant to the reported differences between patients and non-patients (Pivik, 1979-b).

Usual dosage schemes of antipsychotic medication do not seem to have much influence on the quality of eye tracking. Barbiturates have a disfupting effect on smooth pursuit eye movements (Rashbass, 1961; Holzman, Levy, Uhlenhuth, et a1., 1975), like chloral hydrate (single dosis) (Holzman, Levy and Proctor, 1976), ethylalcohol (Corvera, Torres-Courtney and Lopez-Rios, 1973) and perhaps benzodiazepines (Corvera et al., 1973), although Holzman et al. (1975) found no significant effect of single doses of diazepam, and Shagass, Amadeo and overton (1974) found no differences in eye tracking before and after drug treatment with minor tranquilizers. There is no evidence that neuroleptic drugs influence smooth pursuit eye mavements. Indirect evidence of this lack of influence came from the demonstration of deviant eye tracking in relatives of schizophrenics. No significant alteration in eye tracking performance in schizophrenic patients was found after they had been removed from medication for 10 to 14 days (Holzmen, Proctor and Hughes, 1973; Holzman, Proctor, Levy, et al., 1974), and Shagass, Amadeo and Overton (1974) found no significant differences in any of the eye tracking patterns of patients before (medication removed for 4 to 14 days) and during readministration of drugs. Drugs in these studies were usually phenothiazines, but also tricyclic antidepressants, lithium carbonate, minor tranquillizers and methylphenidate. Single doses of chlorpromazine produced no significant changes in eye tracking performance in nonmpatient subjects in a study of Holzman, Levy, Uhlenhuth, et al. (1975). Interestingly, the subjects in this otudy experienced extreme drowsiness and lethargy following administration of secobarbital, diazepam and chlorpromazine, but only the barbiturate disrupted their smaath pursuit eye movements, so this effect seems not the result of sedative actions.

Scoring methodology has been arbitrary and unreliable in the first investigations. Holzman and his co-vrorkers (Holzman, Proctor and Hughes, 1973; Holzman, Proctor, Levy, et al., 1974) used qualitative 
and quantitative scores. Besides the normal eye movement registration they recorded the first derivative of this signal, which reflects the instantaneaus velocity of the eye. The first quantitative score consisted of the number of times the eyes stopped their pursuit of the target, called velocity arrests. A second quantitative score recorded the number of times velocity exceeded the maximum speed of the target by $331 / 3$ of, called positive velocity errors. This second measure has been dropped in later investigations, because of unreliability for technical reasons. The pursuit arrest score has been used by several investigators, but appeared not well correlated in two methods, electro-oculography and infrared reflection. The reason seems to be that it depends on filtering chracteristics of recording instruments and is probably confounded by biologic noise (Lindsey, Holzman, Haberman and Yasi11o, 1978; Iacono and Lykken, 1979-b). This score has been dropped too in later studies.

Recently, new objective methods have been developed for scoring the quallity of smoath pursuit eye movements. Lindsey, Holzman, Haberman and Yasillo (1978) devised a scoring procedure that expresses the goodness of fit of the recorded wave form with the ideal sinusoidal wave form characterizing the perfectly smooth purauit eye tracking pattern, as a signal to noise ratio. The natural logarithm of this ratio was advocated by the authors as a quantitative statement in agreement with qualitative evaluation of the pover spectra. The high correlation between this quantitative scoring method and qualitative judgement was confirmed by Levin, Lipton and Holzman (1981). A related scoring procedure was developed by Iacono and Lykken (1979-a; b; Lykken, Iacono and Lykken, 1981).

Deviant smooth pursuit eye movements have been explained as the result of a dysfunctioning of attentional mechanisms, but the evidence is somewhat ambiguous. Shagass, Roemer and Amadeo (1976) shoverd that eye tracking may be improved by providing periodic changes in the target and asking the subject to monitor these changes. Subjects are for instance required to read changing numbers placed on the target. This effect was replicated by Holzman, Levy and Proctor (1976); Iacono and 
Lykken (1979-a; b) and Levin, Lipton and Holzman (1981), but Pivik (1979) found the opposite effect: the task to detect brief interruptions of the tracking light resulted in significantly more tracking errors in psychiatric inpatients with varying diagnoses. When this procedure results in improved eye tracking, differences between schizophrenic patients and normal subjects still remain significant. Subjects do not realize the improvement of their performance. When there is an improvement it consists of a diminishing of irregularities, which, however, persist in a lesser degree. Individual differences between subjects are preserved (Iacono and Lykken, 1979-a). Those who improve most have the poorest eye tracking baselines. A ceiling effect like this is of course to be experted.

Brezinova and Kendell (1977) reported that distraction of attention in normal subjects and lawering their attention through fatique or boredom produced abnormal eye tracking patterns indistinguishable from those observed in schizophrenics. However, they tried several potentially distracting tasks and only the most difficult (subtracting 13 from 200 serially and writing down each number in the series) produced this disrupting effect. Impairment of attention was produced by prolonged eye tracking (half an hour). Increased anxiety or heightened arousal under a 'threat of shock' condition did not significantly influence eye tracking. The counting backwards procedure, but without uriting, was replicated by Acker and Toone (1978). They too found significantly impaired eye tracking in normal subjects, the degree of impairment increasing with the difficulty of this competing task. Pass, Salzman, Klorman, et al. (1978) also studied eye tracking performance of visually and auditively distracted schizophrenic patienta (with poor eye tracking) and nonpsychotic patients (with better eye tracking). Simple types of distraction did not modify eye tracking quality in either of these groups, but a dichotic listening task decreased the quality of eye tracking in both groups of patients. Solving syllogisms as a distracting task was reported by Tomer, Mintz, Levy and Myslobodsky (1980) to deteriorate eye tracking performance in schizophrenic 
patients. An additional finding of these investigators was the recording of significantly more irregularities in tracking when following the target to the left, as compared to rightuard following. This asymmetry was not found in one nomal control group, but was also observed in a second control group which appeared to rely more on the left hemisphere on the conjugate lateral eye movement test. This asymmetry was interpreted as support to the hypothesis of left hemisphere hyperreactivity in schizophrenia. Hawever, as both hemispheres probably participate in the generation of smooth eye tracking in both directions, the significance of the tracking asymmetry seems ambiguous at the moment. The relevance of disordered eye tracking under distraction conditions to the understanding of psychotics performance on this task seems questionable in the light of the results reported by Lipton, Frost and Holzman (1980). These investigators found impaired eye tracking cycles under distraction in normals, but in a setting of atherwise intact cycles of pursuit. In this respect these recordings were different from those of schizophrenic patients. The influence of voluntary attention on tracking performance of schizophrenic patients was questioned already by Holzman, Proctor and Hughes (1973), who showed that a realerting instruction 30 seconds after initiation of eye tracking did not significantly improve performance of schizophrenic patients. This lack of effect of realerting was replicated by Shagass, Amadeo and overton (1974) and Pivik (1979).

Simple reaction time performance during tracking was shown by Pivik (1979) not to differentiate along eye tracking dimensions, although it differentiated among patient groups. Neither correlated the amount of alpha activity in the EEG with differences in eye tracking performance. It is interesting that Iacono, Tuason and Johnson (1981) found a relation between eye tracking and a manual tracking task, but found essentially normal saccadic latencies, a kind of ocullomotor reaction time task, in schizophrenics.

In the opinion of Holzman and collaborators (Holzman, Proctor, Levy, et a1., 1974; Holzman, Proctor and Levy, 1976; Holzman and Levy, 1977; 
Holzman, Levy and Proctor, 1978) and other investigators (Iacono and Lykken, 1979; Pivik, 1979) the eye tracking dysfunctioning in schizophrenics and other psychotics cannot be attributed to failures in the deployment of voluntary attention. In their opinion the more than once demonstrated improvement of the quality of eye tracking by the mobilization of voluntary attention by a superimposed cognitive task (e.g., number reading) does not imply that motivational variables like voluntary inattentiveness are the principal cause of the eye tracking dysfunctions in schizophrenics. In these cases significant differences between schizophrenics and normals remain. Besides, the mere fact that their eyes are following the target implies that these patients are paying attention. Once the subject looks at the target, the pursuit system is triggered and the eyes can be considered to follow more or less automatically.

Holzman and co-workers (Holzman, Levy and Proctor, 1976; 1978; Holzman and Levy, 1977) have distinguished two principal kinds of deviant smooth pursuit eye movements. In their type I the smooth pursuit patterns are to a large degree replaced by saccadic eye movements. In type II the general form remains intact, but the record is characterized by superimposed small amplitude aberrations, giving the tracings a 'cogwheel', 'sawtooth', or 'spikey' appearance. An extra cognitive task like reading numbers while following the moving target has been shoun to significantly improve type I eye tracking, while type II improves only slightly. Holzman et al. posited that both types of traoking dysfunctioning represent disorders of nonvoluntary attentian, because the deviancies occur in spite of the intention to follow. In a study of normal subjects Iacono and Lykken (1979-a; b) affirmed the existence of these two types of deviant tracking and found a positive correlation of these tracking patterns within individuels. In their opinion an involuntary attention dysfunction seems unlikely, however, because they saw small amplitude spikelike aberrations also appear during saccadic fixation pauses and when the eyes were closed. In addition, saccadic interruptions like the type I deficit seen in schizophrenics also appear to arise in normal subjects as target speed is incremented. 
The type II deficit may be partly the result of superimposed alpha activity in the electro-oculographic record, as these 8-10 $\mathrm{Hz}$ spikes are not found in infrared reflection recordings (Iacono and Lykken, 1979-c). This interference has not been directly assessed, and Pivik (1979) did not find a correlation between alpha activity and eye tracking, but spectral analyses of the oculogram and concurrently recorded EEG has suggested that the 'spikey' rhythm ma represent EEG activity (Iacono and Lykken, 1981). Nevertheless it should be remembered that the same authars found excellent correlations (fram. .89 to .99) between computer derived electro-oculographic and infrared scores.

In conclusion, deviant smooth pursuit eye movements represent a partly genetically controlled trait, related to the schizophrenia concept. The role of attention dysfunction in deviant eye tracking needs further clarification. Part of the variability of eye tracking in schizophrenics may be the result of differences in attention. This part probably largely coincides with Holzman's type $\mathbb{I}$ deficit. Another part, roughly the type II deficit minus alpha interferences, may be mainly the result of underlying deficits in oculamotor or probably general central nervous system integrative or inhibiting functions. However, this distinction is rather arbitrary. Integrative, excitatory and inhibitory processes are the neurophysiological basis of attentional processes. Perhaps smooth pursuit eye movements, as a controlled processing task, should be considered a motor equivalent of the more traditional mainly cognitive sustained attention tasks, that only require motor responses at certain moments, mediated by cognitive, decision making processes. It is unlikely that the source of the smooth pursuit deficit lies in peripheral eye-control mechaniama. The cerebral cortex seems particularly important (Iacono, Tuason and Johnson, 1981; Latham, Holzman, Manschreck and Tole, 1981). The relations: between this indicator of psychopathology and other objective measures clearly deserves investigation in more detail. 


\subsubsection{Reaction Time: Simple (Mean) Reaction Time and Reaction Time Redundancy Deficit (Crossover Phenamenon).}

Performance on reaction time tasks is a well known research area in the study of schizophrenia. Reaction Time is the time between the presentation of a stimulus and the response of the subject to that stimulus - usually pressing a button or lifting the finger from a respanse key. Schizophrenics are generally slower than normals in simple reaction time (when the same response is required on each trial), and they show greater intrasubject and intersubject variability than normalo (e.g., Shakow, 1963; extensive review in Nuechterlein, 1977). The literature on simple reaction time performance is voluminous and will not be discussed extensively here. Historically, it was one of the earliest experimental procedures in the study of psychological processes. Suffice it to refer to one nineteenth century report in which the slowness of response of psychotic patients was already mentioned. In 1874 obersteiner already referred to attentional deficits to explain his reaction time findings: 'Eine Verlangsamung der Reactionszeit ist bei den meisten Kranken mehr oder minder vorhanden (...). Erhöhung der mittleren Reectionszeit, einhergehend mït grösseren Differenzen der Einzelnen Reactionswerthe untereinander, sowie auch gegen das Minimum, ist, wie dasselbe sich auch in den Versuchen mit geschwächter Aufmerksamkeit an Gesunden zeigte, ein Zeugniss von Abnahme der zur Concentrirung der Aufmerksamkeit nöthigen Willenskraft, oder vom Ueberwiegen anderer Voratellungen".

Besides the slowness in response, two additional characteristics of reaction time performance of (schizophrenic) psychotic patients are the greater variability then among normal controls, and the high correlation with elinically rated severity of disorganization (Nuechterlein, 1977). These additional characteristics are true for psychomotor performance of psychotic patients in general (King, 1975). Reaction time is merely the most simple test of psychamotor response. Eye tracking performance is another example. Nuechterlein, referring 
to reaction time studies of schizophrenics, called these studies "a microcosm of psychological research in this disorder, if not of psychopathology generally. Nat only is their number probably larger than those employing any other single experimental task, but the diversity of assumptions and theoretical persuasions that are represented within their boundaries is also inmense" "Neurotic patients have been found to be generally similar to normal subjects in reaction time speed when tested under ordinary conditions, but under psychological or physiological pressure their psychomotor performance deteriorates more than in normals (discussion in King, 1975).

As subjects are assumed to be about equal in performing the motor response (Weiss, 1965; Botwinick and Thompson, 1966; Yates, 1973; Nissen, 1977), differences in reaction time are considered to be largely due to the central (premotor) processing component of the total reaction time. Wishner, Stein and Eastrel (1978), studying central processing stages in a simple reaction time paradigm (stimulus encoding, serial comparison, binary decision, translation and response organization), have reported a lack of specific schizophrenic deficits at the first three of these stages (testing the fourth stage was considered unsuccessful). These findings are consistent with a general central processing dysfunction in psychotic (schizophrenic) patients.

The basic form of the usual reaction time task has three primery stimulus features: an alerting or "ready" signal (warning stimulus) designed to announce the beginning of each trial, a delay interval. or preparatory interval (PI) during which the subject has been asked to wait vigilantly for the second stimulus, which is a signal-to-respond (imperative stimulus).

The preparatiory interval has been found to be an important source of variance. Studies have shown that chronic schizophrenics gemerally differ from normals in the degree they take advantage of regularity of the PI duration, when a series of reaction time trials is: 
presented. Regularity means some degree of certainty about the PI coning next. For many chronic schizophrenics the presentation of a regular series (a repeated presentation of a given PI duration) has been shoun to yield a faster performance than the average of the same PI delivered in random order (mixed with other PI durations), only when the PI's are of short duration. The advantage of regularity seems to be lost for PI durations longer than about 4 seconds. Normals seem to utilize the information provided by regularity and perform faster in regullar than in irregular series for PI"s up to 20 seconds (Rodnick and Shakow, 1940). Although ald people (65-81 years old) have been found to lose the advantage of regularity for PI's longer than about. 10 seconds (Botwinick, Brinley and Pobbin, 1959), this PI duration is still much longer than that reported to be found in most chronic schizophremic patients. When the series is regular and the PI is longer than approximately 4 seconds chronic schizophrenics have been shoun not only to fail taking advantage of the regularity, as normals apparently do, but they perform more poorly than if it were an irregular eeries (Bellissimo and Steffy, 1972; 1975; Steffy and Galbraith, 1974). Because plotting the data results in a crossover af the regular and the irregular functions, this has been called the crossover phenomenon. The information inherent in regularity seens to impair the reaction time performance of many schizophrenics, especially of chronic, process schizophrenics. For this reason this phenamenon was labeled 'redundancy associated deficit' (or "redundancy deficit') by Bellissimo and Steffy (1972). Both terms - crossover phenomenon and redundancy deficit - are used interchangeably here, as is customary in the current literature.

The crossover phenomenon has appeared independent of the general level of reaction time. Reducing process schizophrenics' reaction time to normal levels by a training procedure has been shown not to influence the crossover phenomenon (Steffy and Galbraith, 1980). In relatives of schizophrenic patients who demonstrated the crossover phenomenon, a normal mean reaction time has been found (Cromwell, DeAmicis, Hayes and Briggs, 1979; DeAmicis and Cromwel1, 1979). The independence of slowness of response and crassover was also shown by 
0lbrich (1972) in an investigation of bxain-damaged patients with an overall level of reaction time comparable to that of chronic schizophrenics. The brain-damaged patients did not show any sign of crossover.

Reaction time also differs as a function of the PI on the immediate preceding trial (the preceding preparatory interval or PPI). Schizophrenic patient's performance seems more influenced by the PPI than the performance of normals (Zahn, Rosenthal and Shakow, 1963). When the PPI is greater than the PI (a long PI followed by a short one), the reaction time is slower. When the PPI is less than the PI (a short PI followed by a long one) the reaction time of the latter is relatively faster. Schizophrenics have this PPI effect more than normals. The explanation of this effect in Shakow'a siet terms can be stated as an inability of the patients to recaver from the effects of a set produced by a long PI trial independent of the performance an that trial (Zahn, Rosenthal and Shakow, 1963). This seems to provide an explanation of the crossover phenomenon. In an irregular series a long PI has a high probability of being preceded by a shorter one, and vice versa. The curve of the reaction time as a function of the PPI in an irregular series vould thus be effected in a direction consistent with the crossover phenomenon (Cromwell, 1975). However, when the PPI equals the PI, responses are fastest (Nideffer, Neale, Held, et a1., 1971). This fact should override the PPI - PI inequality and still give advantage to a regular series, but the crossover phenomenon is contrary to this expectation. It seems that for schizophentes whever short-term gain is realized from the influence of the previous trial is lost over the next tala. On the ane hand, resction time performance is enhanced if the innediately succeeding trial has the same PI. On the other hand, speed of reaction the is impaired it hav trial has been preceded by the aame I on a nuber of previous trials.

This reguarity or redundancy effect has been investigeted by Bellissitu and Stefy (1972). They showed that in interpolated ser 100 of Tour regular PI trils with a long urregular series, independent 
of length of PI, the reaction time at first improves and than becomes progressively worse. It is as if after the discovery of regularity after an irregular series, 'the window shades come down on the attentional focus of the process schizophrenic so that the outside world would be less and less adequately responded to" (Cromwell, 1975).

Shakow (1940; 1962; 1963; 1971) interpreted the difference in the schizophrenic's capacity to profit from the predictability as an indication of an attentional dysfunction. He introduced the concepts of major and minor set. In his view schizophrenic patients have difficulty to maintain a major set. By that he means 'difficulty in keeping up a state of readiness for response to a coming stimulus, the state which facilitates the optimal response called for by a given situation' (Shakow, 1962). The schizophrenic patient is considered subject to intrusion or interference, to irrelevant associations: 'change distractors from the enviroment, irrelevancies from past experience' (Shakaw, 1962). These interferences are called minor sets. But Shakow's theory does not satisfactorily explain the crossover phenomenon. It states that the longer duration irregular trials are more frequently under the influence of a major task-set orientation than the langer regular trials, because of the 'reminder' value of the preceding short PI's in the irregular series. However, Bellissimo and Steffy (1975) found that neither eliminating shorter trials preceding the irregular test trials (expected to dampen the crossover phenomenon) nor loading the series with short duration trials (expected to increase the crossover phenomenon) substantially influenced the extent to which long regular trials were slawer than long irregular trials. These results are contradictory to this set theory explanation of the crossover phenomenon. The slower performance for long PI regular series than for long PI irregular series could be explained by drifting avay to irrelevant stimuli, in set terms: intrusion of minor sets. However, as Nuechterlein (1977) states, this seems an example of the ability of set theory to be adapted to almost any outcome in reaction time research, a property deserving criticism and making it a weak theory. 
Another explanation of the crossover phenomenon states that the regular trials are impaired relative to the irregular trials; because the redundancy of information generates an inthibitory process (Bellissimo and Steffy, 1972). However, several investigators failed to find evidence for a gradual sloving of reaction time within a regular series (for a discussion: Nuechterlein, 1977). Tizard and Venables (1956) did not find an increase in reaction time in regular series in chronic schizophrenics, but the crossover phenomenon was present. Nevertheless, the role of an inhibitory process has gained support from an experiment by Steffy and Galbraith (1974). They reasoned that reactive intibition can be expected to dissipate with the passage of time, so the crossover, according to this hypothesis, should become greater with shorter intervals between trials. If crossover is the result of interference with the maintenance of major set, hovever, then it should become greater with longer intertrial intervals. Steffy and Galbraith examined crossover in process schizophrenics with 2 and $7 \mathrm{sec}$. intertrial intervals. They found a greater crossover pattern for the 2 sec. than for the $7 \mathrm{sec}$. condition. This result supports an inhibitory proces concept over a set theory explanation. But, although reaction time at PI's of 3 and $7 \mathrm{sec}$. was slower with decreased intertrial interval, at $1 \mathrm{sec}$. PI reaction time tended ta get faster. One would expect the opposite, since the effects of inhibition can be expected to be most apparent at the fastest stimulus presentation. Perhaps Shakow's set theory is the best explanation of some aspects of schizophrenic reaction time performance, like the forementioned, while the inhibitory process explanation accounts for the crossover phenomenon (Nuechterlein, $1977)$.

There are indications of more general detrimental influence of early, presumably helpful and informative signals on the reaction time performance of (process) schizophrenics. A high redundancy condition, for instance, provides such information. Other examples are knowledge of the PI and responsibility for setting the length of the duration (Cromuel1, Rosenthal, Shakov and Zahn, 1961; Steffy and Galbraith, 1975 and in Mo, unpublished, cited in Steffy, 1978). 
Steffy and Galbraith (1975) examined the variation of the influence ower time of an infrequently occuring noninformative visual probe atimulus. The temporal course of impairment appeared to follow an U-shaped patterr. In this study the group of schizophrenic patients was divided in process and reactive schizophrenics, based on their Elgin Prognostic Scale Scores. Process schizaphrenics shoved impairment at trials with a 1 sec. PI and at 9 sec. trials, with a mid-range recovery. Reactive schizophrenics were found impeired only on trials with a PI of $1 \mathrm{sec}$. This is consistent with the concept of a protective inhibition to manage the unexpected probe stimulations. This inhibitory response can be hypothesized to rise slouly and to overshoot the required level of inhibition needed at longer PI durations. The explanation offered by these authors thus implicates, in line with Epstein's (1966) theory, that process schizophrenics suffer from an inadequate modulation of inhibitory control mechanisms, a kind of all-or-none inhibitary reaction. This inhibitory defect may result in impaimment of reaction time performance linked to a temporal course of information processing.

In the foregoing discussion the term 'schizophrenic' has been used rather indiscriminately. What kinds of patients are characterised by the crossover phenomenon? Unfortunately, so far diagnostic criteria of schizophrenia are almost completely lacking in the studies of the reaction time redundancy deficit. Shakow, in his original investigation (Rodnick and Shakov, 1940) investigated 'schizophrenic subjects (...) hospitalized for periods varying from one month to several years'. The phenomenon seems particularly characteristic of process schizophrenic patients. Some investigations noted the crossover phenomenon only in the farm of a nonsignificant trend in this direction. Considered below are studies that found a statistically significant confirmation of the crossover phenomenon or redundancy deficit, whether measured by long regular and irregular series, or short interpolated regular series vithin a long irregular series. 
Belissimo and Steffy (1972) examined female schizophrenic patients, all receiving a medication of phenothiazine derivates. Diagnostic criteria were not mentioned. These patients were separated into process and reactive groups using the Elgin Prognastic Scale. There vere two control groups, one consisting of nonschizophrenic patients, carrying diagnoses mainly of neurosis and personality disorder, and the other consisting of normal. volunteers. Only the process group showed a significant interaction between PI duration and PI regularity.

The subjects of Steffy and Galbraith (1974) were male schizophrenic patients, who were almost all an some form of psychotropic medication. Again diagnastic criteria vere lacking, but only process patients with lhigh scores on the Elgin Scale were included. There was no control group. A significant interaction between PI duration and PI regularity was found. Bellissimo and Steffy (1975) studied female process schizophrenic patients, without specified diagnostic criteria, but classified as process according to the Ullmann-Giovannani 5cale. All patients received psychotropic medication. There was a normal female control group. Only the patient group showed a significant interaction between PI duration and PI regularity. DeAmicis and Cromuel1 (1979), studying 70 process schizophrenics (diagnosis based on DSM-II criteria and an Elgin Prognostic Scale score of 25 or more, medication not mentioned) and 41 normal controls, also found significant differences between both groups at the 7 secondo $P I$.

Zahn and Rosenthal (1965) found in acute schizophrenics a crossover at the level of 15 seconds PI's, which is far from the range characteristic of process schizophrenics, but is somewhat shorter than found in non-elderly normal subjects. The crossaver phenomenon is not found in all process schizophrenic patients. In several studies about three quarters of these patients show the phenomenon (Bellissimo and Steffy, 1972; 1975; Steffy and Galbraith, 1974), in contrast with about ten percent in normals in the study of Bellissimo and Steffy (1975). In the reactive schizophrenic group in the Belliseimo and Steffy (1972) study one third also manifested a crossover of regular 
Steffy (1980) has presented a summary of the findings in all his studies on redundancy deficit (crossover phenomenon), comprising data on 280 process schizophrenics, 36 reactive schizophrenics and 36 nonschizophrenic paychiatric patients. In these studies the 'embedded set" procedure was employed, in which sets of four regular long PI duration ( 7 seconds) trials are embedded in a quasi random series. Redundancy deficit scores consisted of the reaction time of the fourth regular trial minus the reaction time of the first regular trial, averaged aver the embedded sets. Reactive schizophrenics and nonschizophrenic patients appeared to have as wide a distribution af redundancy deficit scores as process schizophrenic patients, but the percentage of subjects with a positive difference score vas much smallex in the reactive and non-schizophrenic groups. These percentagea were for process schizophrenics $71 \%$, for reactive schizophrenics $39 \%$ and for non-schizophrenic patients $42 \%$. The mean difference scores for these groups were $41.5 \mathrm{msec},-21.1 \mathrm{msec}$. and 4.0 misec. Of the normal cantrols (a total of 94 , put together from several studies) $36^{\circ}$ had a positive difference score, the mean score being -10.1 msec. Including more recent and partly unpublished studies, thus reaching a tatal of 192 normal subjects, $45 \%$ of normals appeared to show redundancy deficit.

The separation of process schizophrenics from other categories improved, however, when a more severe criterion for the definition of redundancy deficit vas used, namely a positive difference score of more than $25 \mathrm{msec}$. This higher cutting point has also been advocated by DeAmicis and Cromuel1 (1979). According to this criterion, 53\% of 288 process sohizophrenics showed redundancy deficit, in contrast to $25 \%$ of 36 reactive schizophrenics, $28 \%$ of 36 non-schizophrenic psychiatric patients and $14^{\circ}$ of 192 normal subjects. It seems a major shortcoming of this criterion, however, that it is not carrected for differences in duration of reaction time. As psychotic patients are well known to be slower in reaction time, a fixed difference of, say, 25 msec., is more easily found in this group of subjects than in non- 
psychotic psychiatric patients or normal subjects.

Studies of remitted schizophrenic patients are scarce. Strauss, Bohannon, Kaminsky and Kharabi (1979) studied schizoplirenic outpatients who were in varying degrees of remission (all had been hospitalized for more than two years) and still received medication. These patients showed a crossover pattern, independent of diagnosis (criteria quite similar to the Research Diagnostic Criteria vs. DSM-II criteria), age, premorbid functioning (Ullmann-Giovannoni score). global adjustment score, or presence of core symptoms.

Marcus (unpublished doctoral dissertation, cited in Garmezy, 1978) found that children who vere presumed to be at risk for schizophrenia by having a schizophrenic mother did not differ from controls on the set index, a measure employed to maximize the crossover phenamenon. Asarnow, Steffy, MacCrimmon and Cleghorn (1978), however, found a tendency for children of schizophrenic mothers to show redundancy deficit. Their mean reaction time tended to be slower in both studies, although in the Asarnow et all. study the difference from controls was not statistically significant.

DeAmicis and Cromwell (1979; Cromwell, DeAmicis, Hayes and Briggs, 1979) examined first degree relatives of thase process schizophrenic patients who showed a crossover effect. Some showed current or past psychopathology and were eliminated from the study. The crassaver in the remaining relatives significantly exceeded the mean negative crossover value of normal control subjects with no history or family history of psychiatric disturbance. However, anly when a difference between the first and the last trial of the interpolated sets of four regular ( 7 seconds PI) trials exceeding +25 maec. was taken as the discriminating point was the difference significant. The magnitude of the relatives' crossover was not as great as that of the schizophrenic patients, but their mean reaction time was as fast as that of normal controls. Thus, the small crossover effect in the relatives found in this study was related to neither manifest psychopathology nor slow mean reaction time. 
The atability of the crossover phenomenon or redundancy deficit has appeared good in a group of schizophrenic subjects, retested after 24 hours. All subjects were consistent in showing or not showing the deficit (Bellissimo, unpublished, cited in Steffy, MacCrimmon and Bellisaimo, 1979). In another study by Kaplan (unpublished, also cited in Steffy et al., 1979) $72 \%$ of the subjects were consistent at retesting after a placebo training condition. To evaluate the stability of the redundancy deficit over longer periods, steffy et al. (1979) tested 18 chronic schizophrenics three times over a six-month period. These (out)patiente vere recelving depot neuroleptic medication during that period, and had an average length of hospitalization of $\mathbb{1}^{\frac{1}{2}}$ years. Although there were substantial changes in clinical state across the three assessments (using standardized psychiatric interviews) no significant chenges in redundancy deficit were found. In those subjects who improved most, however, this deficit was less stable. Overall, the state variation did not seem to have great influence, which was interpreted as attesting to the vulnerability-sensitivity of the redundancy deficit.

The only reference to correlations between redundancy deficit or crossover phenomenon and other attention demanding tasks is in Kornetsky (1972), who cited unpublished data, showing an association between poor performance on the Continuous Performance Test and disturbance in segmental set. The deficit in segmental set was not specified, however, nor was the level of aignificance.

The influence of psychiatric medication on schizophrenic reaction time performance seems very limited. Virtually no research supports the impact of neuroleptic medication on simple reaction time of chronic schizophrenics (for a short discussion: Nuechterlein, 1977). In adidtion, Held, Cromue11, Frank and Fann (1970) found no significant effects on reaction time at different PI's and on the PPI effect. Spohn, Lacoursiere, Thompson and Coyne (1977) recorded a lack of influence of phenothiazines on the ratio of mean reaction time for long PI's, irregular order, to mean reaction time, long PI's, regular order. This ratio is a measure of the crossover phenomenon. 
In conclusion, there is evidence that the crossover phenomenon or redundancy deficit represents to some degree a vulnerability-related trait characteristic, that is familial and a salient feature among schizophrenia-vulnerable individuals, relatively independent of the presence of psychotic symptoms. Slowness on simple (mean) reaction time has since many years been recognized as a useful measure of psychiatric disturbance, particularly psychotic conditions. Its relation to the vulnerability construct is uncertain, however. The relations in psychiatric patients of this long-known indicator of psychopathology as well as of the reaction time redundancy deficit, to other measures of attentional performance, needs further exploration. 


\subsubsection{Continuous Perfarmance Test.}

Tests of sustained selective attention, if of long duration also knoun as vigilance tasks, thave proved to be useful methods of testing psychophysiological hypotheses of individual differences. Claridge (1960; 1967), for instance, demonstrated that 'dysthymics' were significantly better than 'hysterics' and than normals on an auditory vigilance task, but psychotic patients were the poorest performers, although they did not differ significantly from "hysterics" in his studies. The most marked feature of the performance of psychotic patients, however, was the wide variability. The auditory vigilance task used by Claridge consisted of a thirty-minute tape recording of random digite read out at the rate of one per second. Interspersed in the series were number of critical stimuli, to be responded to by the subject, consisting of three consecutive odd digits. These signals occurred with an average frequency of one per minute.

Among the first investigators studying sustained voluntary attention were McDougall (1905) and Philpott (1932; 1934). Practical problems in industry and in military situations prompted an expansion of this type of research and led to an extensive literature on vigilance tests (e.g., Buckner and McGrath, 1963; Davies and Tune, 1970; Jerison, 1970). The typical vigilance experiment has a duration of a half hour or longer, with some lasting many hours. Signal stimuli are presented infrequently, at irregular time intervals. Any type of stimulus may be used as a signal, provided it is perceivable by the observer. A test of sustained selective attention that was mare recently developed is the dichotic listening task, in which a single message or channel has to be shadowed or repeated, while a simultaneous message, another channel, has to be ignored. Schizophrenics generally perform less accurate on this task than non-schizophrenic psychiatric patients or normal subjects (e.g., Payne, Hochberg and Hauks, 1970; Korboot and Damiani, 1976). 
A test of sustained attention related to vigilance tasks is the Continuousi Performance Test (CPT). This test differs from typical vigilance tasks in its shorter duration and in the shorter intervals between the critical stimuli. The CPT usually lasts about 5 or 6 minutes and critical stimuli are presented about avery fifth stimulus presentation on the average, sa there is more pressure to respond. The test was developed by Rosvold, Mirsky, Sarason, et al. (1956) as a procedure for the detection and study of brain damage. In later years the CPT has been used to study sustained attention in schizophrenic patients. The CPT involves the rapid presentation of a long series of simple stimuli in a random sequence and at a constant rate. Usually the stimuli are letters or digits. The rate can be varied, but is usually about one every 1 sec. The task of the subject is to press a key each time and only when a critical stimulus is presented. In a typical CPT a critical stimulus appears on the average every fifth stimulus presentation until a total of 50 critical stimuli are presented.

Most often a visual farm is used. Exposure time of the visual stimuli is about 0.1 sec. Auditory forms have also been used (Williams, Lubin and Coodnow, 1959). In two ways the task can be made more difficult. Subjects can be asked to respond to the critical atimuli only when they follow another specified stimulus (e.g., critical stimulus is $x$, only to be responded to when $x$ is precedied by A). Another vay to heighten the probability of errors is the introduction of distraction. Errors of two types are recorded: an omission error (false negative) whenever the subject fails to respond to the critical stimulus; a commission error (false positive) when a response is made to a noncritical stimulus.

The performance of schizophrenic patients on the CPT was first described by Orzack and Karnetsky (1966). The performance of chronic schizophrenics was compared to that of chronic alcoholics and normal volunteers. The schizophrenic patients showed significant impairment on the CPT as compared to both other groups, but only $44^{\circ}$ performed poorer than any of the norma]. subjects. None of the normal of 
alcoholic subjects made an omission score greater than 4\%. None of the ochizophrenic patients received medication. Separation in good and poor performers (criterion: omission score greater or lesser than 4\%) was not related to diagnostic subtype, length of hospitalization, sex or score on the Wechsler Digit Symbol substitution Test. In a further investigation Drzack and Kornetsky (1971) examined the relationships between CPT performance and a number of variables in a large group of chronic schizophrenics. The group was dichotomized in good and poor performers as in the earlier investigation. Poor performers had more mental illness in their families, especially among siblings. This finding was presented as evidence in support of the hypothesis that attention dysfunctioning as measured by the CPT has a genetic basis, although environmental interpretations remained possible. The other variables, including age at onset, duration of hospitalization, degree of psychopathology and withdrawal did not significantly separate good from poor performers. In these two studies criteria for the diagnosis of schizophrenia were not specified.

Acute schizophrenic patients, according to Pass, Klorman, Salzman, et al. (1980), make also more errors on the CPT than nonpsychotic psychiatric inpatients. Both omission and commission scores were significantly higher for the schizophrenic patients in their study. Distraction during CPT performance, consisting of light flashes $3 / \sec$. simultaneously with irregular beats of a metronome, somewhat surprisingly, did not increase error scores for either group. Probably the task was not difficult enough. In this investigation diagnostic criteria for schizophrenia were based on the first rank symptoms and on the flexible system. Exact criteria were not mentioned, but the schizophrenic group had a significantly higher score on both diagnostic systems. Interestingly, the impaired CPT performance of acute schizophrenics in this study was parallelled by a significantly smaller amplitude of the late positive component of the evolked responses to the stimuli. This component is considered to be $a$ cortical measure of the decision making stage of attention and information processing. Wlker (1981) found significantly more omission errors on the CPT under a distraction condition in 
schizophrenics as compared with schizo-affective and affective patients, all diagnosed according to the Research Diagnostic Criteria. This suggest specificity of this attentional measure to the schizophrenia concept as defined by the ROC.

The hypothesis that the CPT measures vulnerability related attention deficits has in addition to the study of Drzack and Kornetsky (1971) received some support from studies of remitted schizophrenics and of children at risk for schizophremia. Wohlberg and Kornetsky (1973) examined largely drug-free female subjects with a history of ane hospitalization for schizophrenia, discharged more than one year prior to testing (diagnostic criteria: 2 of the following: a. Thought disorder, b. Hallucinations or delusions, c. Inappropiate emotional responsiveness, d. Withdrawn, bizarre and regressive behavior). Because a performance deficit was supposed to be more difficult to elicit in these subjects, a more difficult version of the CPT was applied than in the earlier studies. Subjects were to respond only when the critical (visual) stimulus wes preceded by another specified stimulus, and the same distractor condition was introduced as later used by Pass, Klorman, Salzman, et al., (1980). Remitted schizophrenics differed significantly from controls with respect to amission errors under the distraction condition, but without distraction there was only a trend to significance. Commission errors discriminated both groups during baseline and distraction trials, but not during a final distractor-free condition. Poor premorbid (according to the Phillips scale) schizophrenics made significantly more comission errors (a measure first closely analyzed in this investigation) than did good premorbid subjects. The interpretation of this type of error is difficult, however. It may be slowness of response or a lapse of attention and consequently a response to the stimulus thereafter, or confusion in recognition, or a matter of test attitude: a heightened readiness to respond. Omission errors probably have to be explained in two ways: as the result of inadequate attention, shifting of attention caused by distracting events or thoughts, and as the result of slouness in procesaing, provided that motivation is oufficient. 
Asarnow and MacCrimon (1978) studied wisual CPT performances of acute and remitted schizophrenic patients receiving maintenance doses of phenothiazine medication (Diagnostic criteria: history of thought disorder, hallucinations, or delusions). Remitted patients were tested between 2 and 3 months after discharge. Both acute and cemitted achizophrenica made significantly more errors of omission and commigion under the distractor (auditory-voice) and nomdistractor conditions than normal controls. Under both conditions the patient groups did not differ significantly from each other. However, the difference between performance on the distractor and nondistractor conditiona appeared to be greater in the remitted schizophrenics than in the other two groups. Their performance was relatively more impaired by distraction. The results of the last two studies support the assumption that attentional and information processing deficits as measured by the CPT are related to a ralatively permanent individual characteristic or trait, rather than to episodic, psychotic conditions or state. Some additional support has come from studies of children at risk.

Grunebaum, Weiss, Gallant and Cohler (1974) found a greater number of omission exrors in five years old children of schizophrenic mothers compared with offspring of other (non-schizophrenic and schizoaffective) psychotic mothers and of non-psychiatric control mothers, but there were no differences in errors of commission by diagnosis. For six years ald children the differences disappeared. Follow-up testing three years later showed significantly improved performance of the high-risk children, compared with their own previous performance. There were no significant differences with controls now, but this time the selection process had probably favored the children of the healthier mothers (Herman, Mirsky, Ricks and Gallant, 1977). Cohler, Grunebaum, Weiss, et al. (1977), enlarging on the results of Grunebaum et al. (1974), considered both maternal and child performance within diagnostic groups, controlling for intelligence. No significant intergenerational relationships for CPT performance were found within any of the diagnostic groups: schizophrenic, depressed, and normal mothers. Maternal performance nor child 
performance was significantly different between the diagnostic groups, but the children of depressive mothers tended to show greater impairment on the CPT than either the children of the schizophrenic or normal mothers. The number of children in each group vas small, however. A three years follow-up of part of the original cohort showed findings generally consistent with the earlier results (Grunebaum, Cohler, Kauffman and Gallant, 1970). In a study reported by Rutschmann, Cornblatt and Exlenmeyer-Kimling (1977) and by Erlenmeyer-Kimling and Cornblatt (1978) a large group of children, genetically at risk for schizophrenia, made more errors of omission and of commission than did control children. The ages of the children were between 7 and 12 . Both groups performed more poorly under (auditory-voice) distraction, but the decline in performance vas significantly greater in the high-risk group. In the studies of Grunebaum et all. and of Rutschmann et al, adapted versions of the CPT were used, consisting of colors and of playing cards. Asarnow, Steffy, MacCrimmon and Cleghorn (1977; 1978) tested foster children with a maternal history of schizophrenia, a foster control group and a community control group (to control for the effects of foster placement) with a traditional visual CPT. The ages were between 12 and 18. The high-risk group proved to be comparable to the other two groups an this CPT. The reason may have been that distraction was lacking. In fact the high-risk group in this study showed impaiment on ather tests of attention in which distraction was present (span of apprehension and competing voices).

The relation between CPT performance and low platelet monoamine oxidase (MAO) - postulated to be associated with schizophrenie - vas studied by Buchsbaum, Murphy, Coursey, et al. (1978) in normal. subjects. No significant differences in CPT scores were found between low and high MAO subjects. Kornetsky (1972) has cited unpublished data showing an association between poor CPT performance and disturbance in segmental set. This last measure is related to reaction time redundancy deficit, but is not specified in this publication, nor are other details given. This is the only reference to relations between the CPT and one of the other measures of attention and 
information processing studied in the present investigation. In their review of the vigilance literature Davies and Tune (1970) emphasized that little is known about the relationship between reaction time and detection rate during vigilance task performance.

Test performance on the CPT seems reasonably stable over time. Latz (cited by Drzack and Kornetsky, 1971) found a high test-retest reliability ower a two month period in chronic schizophrenics. In children at risk Erlenmeyer-Kimling and Cornblatt (1978) found highly significant intrasubject correlations for the variaus performance indices over a two years period. The test-retest reliability of vigilance tasks generally has appeared to be quite high (Davies and Tune, 1970). The influence of psychotropic drugs on CPT performance is well known. Important for the present investigation are the effects of neuroleptics. In single doses phenothiazine drugs appear to impair CPT performance (Mirsky and Kornetsky, 1964), but after chronic administration of medication performance improves (Orzack, Kornetsky and Freeman, 1967; Kornetsky, 1972; Spohn, Lacoursiere, Thompson and Coyne, 1977). In their study of chronic schizophrenics 0rzack and Kornetsky (1971) found a significantly better CPT performance in the on-medication group than in the off-medication group, which parallelled the degree of psychopathology. Motivational factors do not seem to be of decisive importance. Latz and Kornetsky (1965) compared conditions with and without reinforcement. Even with reinforcement the performance of schizophrenics was still significantly poorer than that of normals. Nevertheless, cooperation of the subject is essential of course.

In conclusion, there are indications that schizophrenic patients with manifest psychotic symptomatology as well as subjects with a history of a schizophrenic episode and children genetically at risk for schizophrenia manifest deficits of sustained attention as measured by the CPT, more especially the distraction condition of the CPT. This supports the hypothesis that the CPT measures characteristics related to the vulnerability to develop schizophrenia. The relations between measures of CPT performance and other measures of attentional 
processes in psychiatric patients deserve more study. 


\subsubsection{Contingent Negative Variation.}

Usually experimental evidence on attention relies on performance measures as indexes of attention "In the brain attention processes are paralelled by changing potential levels, called event-related slow potentials, to be distinguished from the usual EEG rhythms. One example among the event-related potentials of the brain is the Contingent Negative Variation (CNV). This term signifies a slow negative cortical potential shift which accurs during a warned foreperiod reaction time paradigm, beginning following the warning stimulue ( $\mathrm{S1}$ ) and normally terminating after the imperative stimulus (S2), which demands a respons or decision by the subject. The amplitude of the CNV is largest at the vertex of the scalp, with amplitudes in the range of 14-20 uV characteristic for the 'normal' population. The amplitude diminishes slightly at lateral-central locations, but shows marked reduction over temporal regions, and also progressive diminution anteriorly and posteriorly from central regions (McCallum, 1979). The CNV is made visible against the EEG background activity by averaging across a number of trials.

Although extracerebral origins have been suggested in the electrical fields of eye movements or in skin potential changes, the relevance of these possible artefactual origins have been excluded by careful research. Because eye movements can produce potential shifts that influence the CNU, exclusion of confounded registrations has become standard procedure, however. Suggestions about the physiological origins of the CNW have been reviewed by McSherry (1973). In his opinion vascular changes and glial responses should ba considered irrelevant, because the time-course of the potentials is too rapid, and polarity-reversal in the depth is inconsistent with glial responses. He concluded that the CNV can be explained as arising from a combination of prolonged, superficial axodendritic excitatory post synaptic potential (EPSP) volleys and prolonged deep axosomatic inhibitory post synaptic potential (IPSP) volleys, producing superficial depolarization and deep hyperpolarization. He presumed 
that distant centers, cortical and subcortical, supply the steady barrage of inhibiting and excitatory inputs. Hillyard (1973) proposed that the various types of CNW contain a bilaterally symmetrical, diffuse component, possibly under reticular ar non-specific thalamic control, to which is added a process-specific potential distributed across the cortex according to which thalamo-cortical pathways are active. In a recent review Desmedt, Debecker and Robertson (1979) equated the subcortical controlling system of the CNW with the mesencephalic reticular formation. They proposed that the prefrontal cortex, controlling goal oriented sequential behavior, regulates timed levels of activation in the mesencephalic reticuler formation, that in turn modulates potential levels throughout the cortex cerebri. One of these changing potentials, defined according to their controlling. conditions, is the CNV.

Originally the CNV was also called 'expectancy wave', because it was regarded as a correlate of expectancy, developing in the $51-52$ interval (Walter, Cooper, Aldridge, et al., 1964). Later attention, especially selective attention, and arousal have been proposed as the most important psychological/psychophysiological correlates. These concepts were integrated in a two-process theoretical model, proposed to explain the CNV, by Tecce (1972). According to this model magnitude of the CNV is positively and monotonically related to attention and nommonatonically (inverted $U$ ) related to arousal level. other investigators have argued that the CNV is not primerily related to stimulus anticipation (attention, arousal), but represents motor preparation. These seemingly conflicting explanations have been integrated in constructs that stress the non-unitaxy character of the CNV. Tecce (1972) already concluded that CNU and motor readiness potential are phenomena that can bo defined separately, but that in the usual CNV paradigm occur together and give rise to a hytrid wave. The readiness potential ("Bereitschaftspotential") is a negative potential arising in advance of a movement. It is assumed that this potential shift reflects preparation for self-initiated action involving a motar movement (Deecke and Kornhuber, 1977). 
The twom-component CNV model has received widespread acceptance since Loveless and Sanford (1974) and later Gaillard (1977) described an early and a late CNV wave. The early CNW, originating at a latency of $300-350 \mathrm{msec}$, and with a peak latency variously located at about 450-650 meec (Lovelese and Sanford, 1974), $700 \mathrm{msec}$ (Gaillard, 1977) or 1000-1200 meec (Ploolj-van Gorse1, 1980), has the character of an orienting response. Its latency is probably party determined by the duration of the interstimulus interval. The late CNV, which develops. gradually, is contingent an the response and mainly determined by the level of motor preparation. Although McCallum (1979) has warned against a simple equation, because of differences in distribution, the late CMV seems in many respecte similar to the readiness potential. Generally the topographical distribution of the early CNV component is more anterior than that of the late component (Gaillard, 1977). In earlier investigations the two components could not be discriminated, because the usual preparatory interval was $1 \mathrm{sec}$, which is too short and results in interference. Many later studies, including the one just mentioned, use longer intervals, for instance 3 ar 4 sec. In fact, recent research suggests that the CNV may be still more complex. Principal component analysis has revealed more than two independent slow waves, while one factor was recognizably linked to the whole CNV wave form. However, in this study discriminative properties were added to the stimuli (McCallum and Curry, 1981).

The effects of manipulation of attention on the CNV have been studied in various ways. Increasing task complexity has been shown to first increase the amplitude of the CNV, but at higher levels of complexity produce decline of amplitude (MCCallum and Papakostopoulos, 1973). Some investigators have manipulated attention by using distraction and found a reduction of CNV-amplitude in normal subjects (and a greater reduction in groups of psychotic patients) (McCallum and Walter, 1968-a; McCadlum, 1969; Tecce, Savignano-Bouman and Meinbresse, 1976). Reducing the intensity of the second stimulus (S2) to a near threshold level has been shown to produce a rise of the CNV amplitude (Low, Coats, Rettig and MoSherry, 1967; Rebert, McAdam, Knott and Iruin, 1967). In conditions that require a high level of selective attention, 
like auditory signal detection or auditory intensity discrimination, CNV amplitudes have been found to be positively correlated with measures of perceptual accuracy (Wilkinsom and Haines, 1970; Hillyard, Squires, Bauer and Lindsay, 1971), but other investigators could not find such relations in comparable tasks (Järvilehto and Fruh storfer, 1970). Signal detection performance over a prolonged vigilance task has been shown to correlate with CNU amplitude (Wilkinson and Seales, 1978). High overal1 amplitude was found to be associated with reliatively constant performance, low amplitude with a drop in performance from the first to the second half of the test.

Experiments on the correlations between CNV and reaction time have been revieved by Rebert and Tecce (1973), who concluded that reaction time and CNV are for the most part independent. Their conclusion was that, although the virtual universality of negative correlations and the differentiation of CNV amplitude in terms of intra-individual. analysis of fast versus slow reaction time trials suggeste some relationship between CNV and reaction time, the small average magnitude of correlations, the statistical tests used, the individual variations in the presence of significant correlations, and the common occurrence of CNV-reaction time dissociation indicated strongly that the functional significance of the CNV cannot be equated to functions reflected by reaction time variability. Naätanen and Gaillard (1974) and Papakostopoulos and Fenelon (1975) confirmed the conclusions of Rebert and Tecce for within subject correlations. Across subjects the latter investigators found significant negative correlations at vertex-parietal sites, in contrast to varying, positive and negative, nonsignificant correlations at frontal sites.

A relationship between attention and CNV amplitude is certainly suggested by all these studies, but the variability of the experimental results illustrates the complexity of this relationship and again raises the question to which degree the CNV can be regarded an unitary phenomenon. Although the differentiation between early and late CNV components has added to our understanding of the CNV, there is a danger of too much simplification in such differentiation. As 
McCaLlum (1979) puts it: '(...), the attempt to replace one diffuse and generalized effect with two lacalized and slightly more specific effects vould sem to be an exercise of dubious merit. Implicit in such theorizing is the notion that the different functions described are subserved by differently located generators, the activities of which can overlap and summate. On the whole, it would seem more likely that cortical S(low) P(otential) changes reflect a depolarizing process in a complex mosaic of neuronal domains which have been activated by sub-cortical mechanisms. (...) Thus, on the basis of slowly changing woltages and their distribution over the head, it is probably reasonable to draw conclusions about the extent to which different areas of the brain are involved in a given transaction at different pointa in time'.

Almost a 11 research on the CNV in psychiatric states has centered an correlations between CNV parameters (not differentiated into early and late componenta) and paychiatric diagnosis. Usually short S1-52 intervals (about $1 \mathrm{sec}$ ) have been employed. Correlations of performance measures of attention with CNW have hardly been studied. When Tecce reviewed "Contingent negative variation and individual differences' in 1971 he cited only ane study of the CNV in psychatic patients, wich was the study of McCallum and Walter (1968). These investigators compared patients classified as schizophrenic with high anxiety neurotic patients and normal controls. Both patient groups showed significantly smaller CNV amplitudes, and greater amplitude reduction than normals when auditory distraction was introduced. In addition schizaphrenic patients showed an increased variability of the CNN.

Since then, a number of studies has been published on the CNV in psychiatric states, in which two aspects of the CNV received most interest: amplitude and prolongation of negativity after the imperative stinulus. Timsit and co-workers (Timsit, Koninckx, Dargent, et al., 1968 ; $1969 ; 1970)$ were the first who described a sustained negative potential after S2, which they called Past Imperative Negative Variation (PINV). They discriminated between PINV's of short 
(less than $1.5 \mathrm{sec}$ ), medium (1.5-3.5 sec) and long (more than 3.5 sec) duration. 3 of 45 control subjects, 21 of 70 neurotics, 9 of 37 schizophrenics and aII 8 manic depressive patients showed a PINV of medium duration. PINU's af lang duration were found in only 1 control subject and 3 neurotics, but in 25 schizophrenic patients. unfortunately, like in most of these studies, diagnastic criteria are lacking in their reports. Small and Small (1971) confirmed these findings and found PINV's almost restricted to schizophrenic patients. In later reports an large series of patients Timsit-Berthier, Delaunoy, Koninckx and Rousseau (1973) (also in: Timsit-Berthier, 1976) did not use the term schizophrenia and only discriminated between normal, neurotic and psychotic categories. A significant correlation of PINU's with psychoses was found. Discrimination between types of psychosis on the basis of CNV typolagy was not posaible. Three typies of abnormal duration CNV's (PINV's) were described: typo II, in which a slow return to the baseline begins at the imperative stimulus, type III with a curve 'en plateau', and type IV with a curve 'en deme". Types III and IV appeared specifically associated with psychotic disorders. This was confirmed for schizophrenic psychoses by Fukui, Nakamura, Kadabayashi and Katoh (1978).

Dongier, Dubrovsky and Garcia-kill (1974) (also in: Dubrovsky and Dongier, 1976) found a PINV (defined by these investigators as a prolongation of the negative potential for more than 2 sec after 52 ) in 15\% of normals, 37\% of neuratic patients and $75 \%$ of psychotic patients. This type of PINV was nearly constant in psychoses of less than a half yoar duration, whereas in more chronic psychoses the incidence diminished. An additional funding was an apparent significance of the PINV as an indicator of poor prognosis in neurotic patients. Chouinard and co-workers (Chouinard, Annable, Dubrovaky and Dongier, 1975; Chouinard, Annable and Dongier, 1977) found PINV's (persisting negativity for more than 2 sec) in $64 \%$ of chronic schizophrenic patients. No relationship was found between PINV and sex, age or length of hospitalization. However, schizophrenlics with PINW's, when compared with schizophrenics with ohorter duration potentials, scored significantly higher on the emotional withdrawal 
factor of the Brief Psychiatric Rating Scale, and significantly lower on guilt feelings and anxious depression factors. This suggested an association between degree of post imperative negativity and poor prognosis type of schizophrenia.

On the other hand, McCallum and Abraham (1973) found only a minimal negative shift after 52 in psychotic patients with first rank symptoms present at the time of testing. In later reparts (Abraham, McCallum, Docherty, et a1., 1974; Abraham, McCallum and Courlay, 1976) the presence of a PINU in schizophrenic patients was confirmed by these investigators, but apparently to a lesser degree than found by, for instance, Timsit-Berthier, Dongier, and their co-workers. However, Abraham et al. found some symptoms, notably incomprehensibility of speech, present in a significantly greater proportion of the group showing prolongation. Verhey (1980) found postimperative negativity significantly larger than in normal controls only in schizophrenics as defined by Class $S$ of the Present State Examination-Catego System (other diagnostic classes in his study included depressives and neuratics). Although Small and Small (1971) did not find postimperative negativity in manic-depressive patients, Abraham, McCallum and Gourlay (1976) found consistently prolonged negativity in patients classified as manic-depressive (current state) according to the Present State Examination. Both studies of Abraham and coworkers made use of this diagnostic system. The criteria for a diagnosis of schizophrenia or psychosis in the other studies are generally obscure. Nevertheless an association of PINV with psychosis has received considerable support, although the phenomenon is not invariably present in, and seems not restricted to, schizophrenia or psychotic disorders in general.

There are some indications that increasing symptom severity parallels an increasing PINV and that clinical progress is associated with a decrease of the PINV. Abraham, McCallum and Gourlay (1976) found some link between PINV and current state in non-psychotic patients, whereas in psychotic and manic-depressive patients this CNV feature appeared rather consistent at retesting. However, for manic-depressive patients 
a relationship with symptoms rather than with diagnosis or trait was found by Rizzo, Amabile, Caporali, et a1. (1979) in a study of CNIV changes along the course of manic-depressive illness. A PINV was very conspicious in the manic phases, but not present in the depresisive phases. Timsit-Berthier, Delaunoy and Rousseau (1976) recorded modifications of the CNV termination mode at retesting in half of their psychotic patients, while normal controls generally performed stable. Generally good stability of the PINV was found by Tinsit, Koninckx, Dargent, et a1. (1969; 1970). Overa11, the data on the stability of the PINU in psychotic patients are inconclusive at the moment. Concerning medication, there are no indications that psychotropic drugs have influence on the PINV. Small and Small (1971) and Abraham, McCallum, Docherty, et al. (1974) based their canclusions on patients withdraun from medication, and Timait-Berthier, Delaunoy, Koninckx and Rousseau (1973) recorded PINV's in 17 of 21 psychotic patients who were not receiving medication.

The case of the interpretation of the PINV has not been settled yet. Timsit-Berthier, Delaunoy and Rousseau (1973) and Dongier, Timsit-Berthier, Koninckx and Delaunay (1973), studying the readiness potential in neurotic and psychotic subjects and normal controls in a design without external stimuli, recording potential shifts preceding and following voluntary movements, found a diffierent type of potential among psychotics, and to a lesser degree also among neurotics, than in normals. In these subjects the negative patential persisted after the end of the motor activity and ended with a more gradual slope. This feature resenbles the PINV in CNV's and suggests that a traditional CNV design is not essential for eliciting prolonged post imperative negativity. However, a motor response is not essential for eliciting a PINV in schizophrenic patients, although the frequency of prolonged CWV'g in non-response paradigms appears lower than under standard conditions (Fukui, Nakamura, Kadobayashi and Katoh, 1978). Dongier, Timsit-Berthier, Koninckx and Delaunoy (1973) found the persisting negativity of the motor readiness potential even more trait-related than the prolonged CNV (as in their study the PINW often parallelled the clinical condition, which did not occur in the case of the 
readiness potential), but Dubrovsky and Dongier (1976) failed to replicate this finding. In their series of psychotics the CNV was more often prolonged than the readiness potential in acute patients, and far more in chronic patients.

Weinberg (1973) studied the potential shifts in a CNV paradigm in which a discriminetive response at 52 was made contingent on information carried in $S 1$ and feedback was given by means of a third stimulus. The data indicated that the CNV was sustained during the period the (normal) aubjects were waiting for feedback, although there was no intention to respond. These findings were confirmed by Peters and Knott (1976). Prolongation of the CNV has also been produced in normall subjects by introducing a random element, in that pressing the button sometimes produces a light flash (Delaunoy, Timsit-Berthier, Rousseau and Gerono, 1975-a; b). In a variation of this experiment, when the imperative tone stimulus occasionally continued for three-quarters of a second and was not terminated by the subject's key press, some normal subjects were shown to develop a post imperative negative potential (Timsit, cited by Callaway, 1978). Gauthier and Gottesmann (1975) produced a prolonged CNV in normal subjects by introducing external interference. When labyrinthine stinulation and mental calculation were applied at the same time a PINV emerged. The presence of only one of these types of interference did not result in prolonged negativity. The other CNV parameters, notably the amplitude, were not significantly changed by these procedures.

The PINV thus seems to be, at least in part, a reflection of the context within which the subject is operating. Warning stimulus and preparatory interval are not essential elements of this context, as a motor response paradigm alone also produces the phenomenon. Uncertainty and distraction seem to be controlling variables of this negative potential prolongation when it accurs in normal subjects. The differing results suggest that, like the CNW, the PINW is a nonunitary phenomenon, with persisting attention as well as motor aspects. Of course the dependence on environmental conditions found in normal subjects and the influence of current state in psychiatric 
patients, found by some investigators, does not exclude the possibility of trait-relatedness in schizophrenic subjects, but evidence on this relationship is still inconelusive.

A second CNW characteristic found in psychotic patients is a diminution of the amplitude of the negative patential in the interstimulus interval. MeCallum and Walter (1968) found significantly lower CNV amplitudes in schizophrenic and neurotic ('chronic anxiety') patients than in normal controls. Auditory distraction during the 51-52 interval increased the amplitude difference between the groups, and in fact made the CNV virtually disappear in the neurotic and schizophrenic groups. In later studies by this research group (McCallum and Abraham, 1973; Abraham, McCallum, Docherty, et a1., 1974; Abraham, McCallum and Gourlay, 1976; Abraham and McCallum, 1977), the presence of a significantly lower CNV amplitude in schizophrenics has been confirmed. In addition, schizophrenic patients who demonstrated first rank aymptoms or vere classified as "Nuclear Syndrome' according to the Present State Examination appeared to have significantly lower amplitudes than psychotic patients without these symptoms. Their lower mean CNV amplitude remained virtually unchanged on retesting and after treatment, when schizophrenic symptoms had disappeared. An auditory distraction condition produced significantly more attenuation of the CNV in the schizophrenic subjects than in normal controls, and more in patients with first rank symptoms than in other psychotic groups.

Small and Small (1971) also found significant differences of amplitude between psychiatric patients and normal controls, but not between various patient groups (schizophrenics, manics and depreasives). However, they included no nourotic group in their study and used a preparatory interval of only $0.5 \mathrm{sec}$. Although in their earlier reports the group of Timsit-Berthier, Koninckx and Delaunoy (1973) mentioned nearly identical mean amplitudes for groups of psychotics, neurotics and nommals, they have later also found generally lower CNV amplitudes in psychotic subjects, while neurotic subjects also demonstrated lower mean amplitudes than normals (Timsit-Berthier, 
Delaunoy, Koninckx and Rousseau, 1973; Timsit-Berthier, 1976). In their large series $8 \%$ of normal subjects, $10 \%$ of neurotics and $24 \%$ of poychotics had a CNV amplitude less than 5 uV. These results were replicated by Fukui, Nakamura, Kadobayashi and Katoh (1978). Verhey (1980) also found a significantly reduced CNV amplitude in schizophrenics (Clase 5 of the Present State Examination - Catego System) as compared to normal controls. Referring the amplitude to a baseline based on the 'take off point' described by Loveless (1973; Loveless and Sanford, 1974) at $350 \mathrm{msec}$. after 51 improved the relationship between amplitude and (increasing) psychotic pathology. However, the 'take off' point of the CNV curve from the evoked potential to SI is not as clear in paradigms using longer interstimulus intervals than $1 \mathrm{sec}$, as used in the study of Verhey. An additional finding was the significantly larger difference of amplitude between central and frontal derivations in normal controls than in any of the patient groups (including schizophrenics, depressives and neurotics).

The attenuation of the CNV in psychotic (schizophrenic) patients does not seem to be the effect of psychotropic medication (Abraham, MoCallum, Docherty, et al., 1974). Concerning stability, Timsit-Berthier and Gerono (1976) found a test-retest correlation for CNV amplitudes of 0.55 for stable patients after 1 to 2 years, and of 0.48 for patients who were improved or worsened after the same time period. Although bath low amplitude and post imperative negativity are CNV features correlated with serious psychiatric disturbance, they seem to lack a clear interrelationship. Timsit-Berthier, Delaunoy and Rousseau (1976) reported that in patients with affective psychoses presenting a CNV with a 'normal' amplitude greater than 5 uV appraximately $56 \%$ had a prolonged CNV, and in schizophrenic patients and paranoics with a 'normal' CNV amplitude $78 \%$.

No studies of the CNV in borderline psychatic patients are known, perhaps excluding an early study of walter (1966) in which he found CNV's of normal amplitude, although of high variability, in schizoid, but not psychotic, individuals. In 1973 Dongier, reviewing the 
literature on correlations between CNV features and psychiatric diagnoses, concluded that there seems to be a parallel between slow potential abnormalities and severity of psychopathology, the incidence of abnormal CNV"s in neurotics generally being half-way between the incidence in normal and the incidence in psychotic groups. Later studies have confirmed this conclusion (Dongier, Dubrovsky and Garcia-Ril1, 1974; Abraham, McCallum and Gourlay, 1976; Fukui, Nakamura, Kadobayashi and Katoh, 1978). The study of Abraham et al. in particular gives a clear illustration of the types of these relationships across a wide range of disturbance. They classified their subjects according to the 36 syndromes of the Present State Examination, and found a relation between increasing syndrome severity on the one hand, decreasing distraction amplitude and increasing post imperative stimulus negativity on the other hand. The research findings on CNV's in psychopathic personalities are diverging. Some investigations showed reduced amplitudes (Walter, 1967; McCallum, 1973), while later investigators could not find significant differences between psychopathic and normal subjects (Syndulko, Parker, Jens, et al., 1975; Fenton, Fenwick, Ferguson and Lam, 1978). In patients with varying psychosomatic disorders higher CNV amplitudes than in normals have been found (Timsit-Berthier, Koninckx, Delaunay and Rousseau, 1972; Timsit-Berthier, 1976), but studies have been scarce and diagnoses unclear; so conclusions should be tentative.

The relations between CNV features and personality traits as psychometrically measured in normal subjects are scarce and confusing. Difference in CNV amplitude was assumed, but could not be found, between groups of high and low scorers on the Taylor Manifest Anxiety Scale under normal conditions (Knott and Irwin, 1967; 1968). Werre, Faverey and Janssen (1975) found a pasitive correlation between extraversion and CNV amplitude (the more extravert, the higher the CNV amplitude), but no relation between neuroticism and CNV. (Personality traits measured by the Amsterdamse Biografische Vragenlijst, the Dutch counterpart of the MPI of Eysenck). In patient. groups, McCallum and Walter (1968) found significant negative correlations between neuroticism scores as measured by the Middlesex 
Hospital Questionnaire and CNV amplitude in a distraction condition. Fukui, Nakamura, Kadobayashi and Katoh (1978), also studying (neurotic) patients, replicated these negative correlations between neuraticism and amplitude, but now under standard conditions. In nonresponse sesaions (no button pressing at 52) they found a high negative correlation between extraversion and CNV amplitude. Personality dimensions were scored on the MPI, Japanese version.

Only a feu experiments have sought to induce changes in the CNV by pharmacological means, and not very much is known of the influence of psychotropic medication on the CNV. The evidence on the influence of dextroamphetamine is ambiguous, varying from no influence to, on the one hand increase, on the other hand decrease of the CNV amplitude (Low, 1969; Tecce and Cole, 1974). Benzodiazepines have been shoun to produce a small reduction in CNV amplitude in normal subjects (Hablitz and Borda, 1973; Ashton, Millman, Telford and Thompson, 1974; Kuda, 1977). However, Lov (1969) reported no apparent effect of chlordiazepoxide on the CNV, and Janssen, Mattie, Plooij-van Gorsel and Werre (1978) found a small (non-significant) increase of the CNV after administration of chlordiazepoxide. The latter authors reported a CNV decrease with caffeine, while Ashton et al. found the opposite effect of caffeine. Single dosis of a neuroleptic drug, chlorpromazine, have been found to reduce CNV amplitude in normal subjects (Tecce, Cole and Savignano-Howman, 1975). Kuda (1977) however found augmentation or decrement in amplitude, depending on the subject and on the modality of 52 . The conclusion has to be that the evidence on the effecte of these various psychotropic drugs is conflicting, and firm conclusions are certainly not justified. As stressed by Janssen et al. (1978), part of the diverging results may be caused by neglect of individual differences, such as degree of extraversion. In addition, the biphasic effect of dextroamphetaraine found by Tecce and Cale (1974) gives an illustration of the complexities encountered in studies like these.

More extensive research has been done on psychotropic drug effects on evoked potentials (reviewed in: Saletu, 1974; Shagass, 1974; Shagass 
and Straumanis, 1978). Psychoactive compounds in the clinical dosage range appear to induce latency and amplitude changes, which are rather typical for the clinically established psychopharmaceutical classes. There is considerable evidence, however, that neuroleptic druge, which induce latency increase and amplitude attenuation when given to normal subjects in single doses or for a short term, tend to normalize dewiant evoked potential characteristics in those psychotic patients who show favorable clinical responses to neuroleptic drug therapy. The same applies to central nervous system excitants in hyperkinetic children (Shagass and Straumanis, 1978). This type of evidence is not directly available on CNV deviations in psychotic patients, but, as has already been mentioned, Timsit-Berthier, Delaunoy, Koninckx and Rousseau (1973) found the same CNV abnormalities in medicated and in unmedicated psychotic patients, and several investigators based their conclusions on experiments with untreated patients and arrived at the same results as experimenters using medicated patients. Finally, the influence of age differences on the CNV seems limited. The CNV remaine appraximately constant (Klorman, Thompson and Ellingson, 1978).

In conclusion, two aspects of the CNV seem characteristic of (schizophrenic) psychotic patients: the amplitude of the CNV is lower than in less disturbed patients and narmals, and there is more persistence of the negative potential after the imperative stimulus. The relevance of these findings to trait rather than state is uncertain at the moment. The relations with other presumably objective indicators of psychopathology have been studied insufficiently. In addition, discrimination between early and late companents of the CNW in psychiatric patients has hardly received attention so far. 

DESIGN AND PROCEDURES

\subsection{GENERAL DESIGN.}

Like most studies of schizophrenia, this research is cross-sectional in nature. Samples were selected from schizophrenic and nomschizophrenic patient and normal control populations. The patient subjects were selected from anong the in- and outpatients of a regional psychiatric hospital. Patients were intervieved by the investigator at the day of their testing in a semi-structured way during about an hour and classified according to psychiatric diagnosis. In addition they were scored on diagnostic measures considered significant to the schizophrenia concept: affective flattening, social isalation, premarbid asociality. Subsequently they received, from an assistent of the department of clinical neurophysiology, the various attentional performance tests and the personality questionnaire. Consecutively they performed the Continuous Performance Test, Simple Version, answered the Eysenck Personality Questionnaire, and performed the Continuous: Performance Test, Complex Version. The Eye Tracking task, the Reaction Time task, and the recording of the Contingent Negative Variation took place next in a randomly varying order in order to prevent fatigue and diminishing movation to selectively influence one of the teats. All subjects were tested in the same setting and under the same instructions. 


\subsection{SUBJECTS: CHARACTERISTICS AND CLASSIFICATION.}

\section{Subject selection.}

The patient subjects were 80 psychiatric patients, partly hospitalized recently, partly non-hospitalized, male and female, from the "Psychisch Gezondheidscentrum Welterhof', a psychiatric hospital in Heerlen. Patients were selected according to their psychiatric diagnosis, so that 4 groups of 20 patients each vere formed. The normal control group consisted of 20 subjects selected from among the hospital personnel as well as from among other volunteers. They had not and never had a psychiatric disturbance to such a degree that they sought professional help for it. To ensure naĩvety, psychologists, doctors, and other professionals were excluded.

Exclusion criteria for patient and normal graups vere:

(a) Age below 20 or above 60 years.

(b) Psychotic before age 20 years.

(c) Subnormal intelligence as estimated on the basis of school history (repeating more than two classes during primary school, which had to be finished).

(d) Regular abuse of alcohol or abuse of drugs acting on the central nervous system.

(e) Clinical evidence of organic brain dysfunction or other somatic disorders to which psychiatric symptomatology might be attributed.

(f) Serious non-correctable impairment of vision, signs of nystagmus or other clinically distinguishable defects of ocular motility.

(g) Obvious impairment of hearing.

(h) Failure to complete all of the attention and performance tests in one session, whatever the basis of the cooperation difficulty. Especially subjects too withdrawn and apathetic, or too excited and acutely disturbed, to cooperate fully in the complete program. In practice, this criterion implied that chronically hospitalized patients were excluded from the samples. Only a very few patients, not restricted to specific diagnostic categories, refused 
participation.

The groups were matched as far as possible on age. Matching groups on social status and on intelligence was considered inappropriate, as schizophrenics are well known to be characterized by lower scores on both variables, and a blas would thus be introduced (discussion, for instance, in Neale and Oltmanns, 1980). Moreover, among the virtues of psychomotor-attentional performance variables Like those introduced in this investigation is their demonstrated independence of inteligigence and socioeconomic status (King, 1975).

Psychiatric diagnosis and other diagnostic measures.

The diagnostic descriptions in this investigation have been discussed in Chapter 5.1. Details on the diagnostic criteria are included in the Appendix. In summary, for each of the subjects five independently assigned diagnostic classifications were determined:

(a) Psychiatric diagnosis according to DSM-III (APA, 1980): schizophrenia, non-schizaphrenic psychosis, schizotypal personality disorder, neurosis, or narmal. This dependent variable is the criterion according to which patients and normal subjects were selected and categorized in five groups of 20 subjects each. The other (independent) diagnostic variables (b-d) did not influence in eny way classification according to psychiatic diagnosis (a), nor did the opposite occur.

(b) Degree of affective flattening, as determined by the Rating Scale for Affective Flattening of Abrams and Taylor (1978).

(c) Degree of social isolation, as determined by the social Functioning section of the Schedule for Affective Disordars and Schizophrenia (Spitzer and Endicott, 1978).

(d) Degree of premorbid interpersonal dysfunctioning, as determined by the Premorbid Asocial Adjustment Scale of Gittelman-Klein (Kokes, Strauss and Klorman, 1977). (As each of the subjects was rated on all seven subscales, the total scare was not averaged as advocated by Gittelman-Klein, see Chapter 5.1.2.).

(e) Psychoticism score as determined by the Eysenck Personality 
Questionnaire (Eysenck and Eysenck, 1975).

The symptom and social functioning based diagnostic measures in this study were determined during a psychiatric interview, using in addition relevant information available from the hospital file. Although the rating scales selected for this investigation serve to increase the reliability of the assessment of the symptomatological and social characteristics, this reliability is likely to be negatively influenced by the problems of retrospective judgement (premorbid asociality) and of contextual influences of the interview situation (affective flattening). These problems could not be eliminated. They could only be pushed back as far as passible, which in fact was tried by chosing these rating scales.

The fact that there was no standardized interview schedule available designed to elicit the signs and symptorns incorporated in the DSM-III criteria might be considered a shortcoming, reducing the reliability of the diagnostic classifications thus arrived at. (Recently, a new interview schedule has been developed, allowing lay interviewers or clinicians to make diagnoses according to the Research Diagnostic Criteria, the St. Louis Criteria, and a selected group of DSM-III categories. It is still undergoing further improvements (Robins, Helzer, Croughan and Ratcliff, 1981)). However, although structured mental state interviews may attain high inter-rater reliability in the hands of trained investigators (Wing, Cooper and Sartorius, 1974), its test-retest reliability has appeared less good (Cooper, Kendell, Gurland, at al., 1972; WHO, 1973). But more important is the concluaion of Ward, Beck, Mendelson, et al. (1962) that only 5\% of diagnostic disagreements resulted from differences among diagnosticians in interview technique, while $80 \%$ of all diagnostic disagreements was the result of inadequate nosology. This last problem is markedly reduced by the clear diagnostic criteria of DSM-III. In addition, diagnostic classification based on DSM-III not only requires assessment of symptoms currently present, and observable and elicitable during the interview, but also requires assessment of temporal variation and duration of symptoms, and of combinations of 
symptoms, not only during the interview or during sone recent period preceding the interview, but also in earlier periods, preceded and/or followed by prodromal or residual symptoms. Applying the DSM-III criteria thus requires a comprehensive approach, reflecting all information available, including information from the psychiatric interview, from contacts with other informants, from the nursing staff and all other informetion available from the hospital file, including earlier observations and interview data.

Several studies have shown that a comprehensive approach based on all information obtainable reveals far more psychopathology than a research psychiatric interview (Carpenter, Sacks, Strauss, et al., 1976; Douning, Francis and Brockington, 1980). It seems that the brevity and perhaps the formality of the standardized interview limits the sample of observed behavior and symptoms elicitable, especially in psychotic patients who are too inconsistent in their accounts of their symptomatology and too variable in their behavior for it to be safe to rely on a single psychiatric interview, although a standardized interview may be the best single source of information on the clinical atate of the patient.

\section{Medication.}

A major problem in the study of psychotic patients is the almost universal use novadays of antipsychotic drugs in the treatment of these patients. The ideal experiment should use patients not receiving medication. Newly contacting paychotic patients not yet using medication are very scarce, however, and psychotic patients in therapy not using medication are almost completely lacking, and likely to be atypica1. Complete excretion of antipsychotic drugs may take periods of at least one month (Forrest; forrest and Mason, 1961). A minimum wash-out periad of two to four weeks is therefore often recommended (e.g., Chapman and Chapman, 1973). Withdrawal from neuroleptic medication thus implies waiting a rather long period, during which the patient may reliapse and develop symptams that necessitate a return to drug treatment. There may also be an increased $\mathrm{r}$ isk for patients 
withdrawn from medication, for instance an increased risk of suicide, or of agression. The clinical prablems thus created, as well as the ethical concerne, have their influence on the ultimate selection of auitable patients, for clinical relapse requiring return to medication leads to the loss of a number of patients to an investigation involving drug withdrawal. In addition, saliciting informed consent from patients for participation in a drug-withdrawal study creates a bias, because individual differences in attitudes can be expected to influence decision making in this self-selection process.

The recruitment of suitable patients is thus biased in various ways, and the testable withdrawal-aurviving sample is likely to differ significantly in prewithdrawal characteristics from the sample nat surviving the selection procesa. In particular, the surviving sample 1.s probably less severely disturbed. The first empirical confirmation of this long suspected bias was the study of Spohn and Fitzpatrick (1980). They performed multivariate analyses of variance involving demographic, organiamic and course-of-illness variables of a rather large population of schizophrenic patients. Their conclusion was that the final consenting and not relapeing sample differed significantly from the reference population from which it was drawn. The most discriminating variables were marital status, occupational skill level, and duration of illness - all individual difference measures related to the premorbid social adjustment dimension. Relapsing patients also performed more poor Iy on the Cantinuous Performance Test before withdrawal of medication.

An acceptable way of studying patients on medication seems continuing this medication, but using an index method, to make drug doses equivalent over different types of drugs. Although drug effects are not removed by this method, any differences as to level of medication between groups of patients divided on variables on interest are made explicit. Venables (1975), for instance, has advocated the use of this method, which is also used in the present investigation. The interaction between psychatropic drugs and variables on interest 
should be examined directly and specifically, as advocated, for instance, by Neale and 01tmanns (1980). The evidence on drug effects on the attentional variables in this study is discussed in chapter 5.2. Generally, neuroleptics seem to improve attention-information processing functions in psychotic patients, or to leave these functions unaffected (e.g., Spohn, Lacoursiere, Thompson and Coyne, 1977). This normalizing influence tends to reduce rather thath enhance differences between psychotic subjects using antipsychotic medication and nompsychotic subjects not using these drugs.

From relevant publications (Haase, 1963; Hollister, 1970; Kinzler, 1971; Lehmann, 1975; Sieberns, 1978) a dosage index was composed for use in this investigation. This index, which expresses dosages of neuroleptic drugs in equivalent doses of haloperidol, is included in Appendix 8. Other types of psychotropic medication were withdraun as far as possible, especially sedatives and hypnotics. These drugs were discontinued at least one week before testing. Lithium and antidepressive drugs were continued.

\section{General characteristics of subjects.}

Characteristics of subjects (age, sex, hospitalization and medication) for the various diagnostic subgroupings are presented in table 2 . 
Tabie 2. Characteristics of subjects.

non-sch.

schiz. psych. sch.typ. neur. norm. total

Variable. $\quad N=20 \quad N=20 \quad N=20 \quad N=20 \quad N=20 \quad N=100$

Age (years)

$\begin{array}{lcccccc}\mathrm{M} & 36.5 & 39.9 & 34.1 & 33.7 & 33.1 & 35.4 \\ \mathrm{SD} & 9.3 & 12.9 & 11.4 & 7.6 & 10.0 & 10.5 \\ \text { range } & 22-57 & 22.59 & 20-59 & 23-45 & 20-54 & 20-59\end{array}$

Sex

$\begin{array}{lrrrrrr}\text { N. males } & 12 & 4 & 9 & 10 & 12 & 47 \\ \text { N. females } & 8 & 16 & 11 & 10 & 8 & 53\end{array}$

Hospitalization

(months)

$\begin{array}{lllllll}M & 11.1 & 5.4 & 3.5 & 1.6 & 0 & 4.3 \\ \text { SD } & 11.8 & 5.7 & 5.2 & 5.6 & 0 & 7.7 \\ \text { range } & 1-44 & 0-18 & 0-19 & 0-25 & 0-0 & 0-44\end{array}$

Medication

(haloperidol eq.)

$\begin{array}{lllllll}M & 6.4 & 5.8 & 1.5 & .3 & 0 & 2.8 \\ 50 & 4.2 & 4.8 & 2.7 & .9 & 0 & 4.1 \\ \text { range } & 0-15 & 0-15 & 0-8 & 0-4 & 0-0 & 0-15\end{array}$




\subsection{PROCEDURES: ATTENTIONAL TASKS.}

\section{(a) Continuous Performance Test.}

In the present investigation an auditory CPT with distraction was used. Distraction is known to increase the discrimination between remitted schizophrenic and nonschizophrenic groups on the CPT (Wohlberg and Kornetsky, 1973) and probably increases the vulnerability-related character of the test. Auditory sustained attention tasks are generally performed better by normal subjects than visual tasks (Davies and Tune, 1970). So optimal performance and vulnerability-sensitivity seem to be combined in an auditory. distraction task. The stimuli to be attended to consisted of 500 digits, pseudo randomly arranged, spoken by a male voice. Critica 1 stimuli (stimuli-to-respond) were 7 and 9 in the simple version, and 7-followed-by-1 and 9-followed-by-1 in the complex version. Distraction consisted of competing information: digits spoken by a fernale voice in the intervals of the male spoken digits, at the same level of loudness. Interstimulusintervals were 1 second. Voices vere tape recorded and stereophonically listened to with cushened earphones at a comfortable level of loudness as preferred by the subject.

After a short instruction trial subjects received the aimple version first. In the simple version 20 per cent of the stimuli (male spoken digits) were critical stimuli, in the complex version 10 per cent. The mon-critical stimuli consisted of a random selection of digits, with the exception of the digit 1 , which was presented twice as often as the other mon-critical digits in order to preserve the complexity of the 7-1, 9-1 condition. The distractor stimuli consisted of the same digits, but in another random order. Details can be found in Appendix-6. These CPT conditions were somewhat more complex than those used by other investigators and resembled to some degree the auditory vigilance task used by Claridge (1960; 1967), but the pressure to respond was higher here, and the duration was limited to nearly $8 \frac{1}{3}$ 
minutes. A more complex version of the CPT vas preferred, because the digtribution of scores with traditional CPT's is rather skewed. Paor performers have usually been defined as thase subjects malking more than 4 per cent omiseion errors. Making the task more difficult was expected to create a less skewed distribution and to lessen the influence of slight 'randam' fluctuations of attention on the score, so that 'real', that is, more enduring deficits are measured. Subjects were asked to press a button, activating a buzzer, each time and only when they heard a critical stimulus. Scoring was done directly by the experimenter, but tape recording were made of the test sessions to aerve for a check up of the original scoring. For buzzer reactions to be accepted as correct they had to start before the next stimulus was finished. Analysed were the number of omissions (false negative responses) and the number of commissions (false positive respanses) for simple and complex versions. These numbers constitute the scores in the analyses except for the omission score of the complex version, which was doubled in order to correct for the factor two difference between simple and complex versions in number of critical stimuli. Applying this procedure to the commission scores was considered inappropriate, as the total number of stimuli was the same in both conditions.

\section{(b) Mean Reaction Time and Reaction Time Crossover Phenomenon}

\section{- Redundancy Deficit.}

Included in the present investigation were three regular series of werned reaction time trials, each consisting of 9 trials with preparatory intervals of 2,4 and 8 seconds, and an irregular series of 27 trials, consiating of trials with the same preparatory intervals, 2,4 and $B$ seconds, 9 of each. Intertrials-intervals vere 4.5 seconds. Intereeries-intervals vere 15 seconds. A 28 th trial, in fact the very first, was added to the irregular series, but was dropped in the final computations. This vas done in order to correct for the PPI (Previous Preparatory Interval) effect. Each preparatory interval duration was repreaented an equal number of times in the preceding intervals of the irregular series. Details are in Appendix 7 . 
The experiment was located in a dimly lighted sound-dampened room. Subjects were asked to prepare for reaction after each waining stimulus (a click, $800 \mathrm{~Hz}, 50$ msec., $55 \mathrm{~dB}$. ) and to press a button with their preferred hand as fast as possible after the imperative stimulus (a $1000 \mathrm{~Hz}$. tone, $70 \mathrm{~dB}$. ended by button pressing). After some instruction trials the complete program was started by the computer (Hewlett Fackard $2100 \mathrm{~S}$ ). Each time the subject pressed too early, before the imperative stimulus started, this trial was discarded and repeated after 4.5 seconds. Computed were the means of the regular series and the means of the same preparatory interval duration trials in the irregular series (excluding the added PPI correcting trial). Computation of reaction time was done on line by analog-digital conversion of the imperative signal, with $1000 \mathrm{~Hz}$. sampling frequency. Redundancy Deficit scores were computed by dividing the mean reaction time of the regular 8 seconds preparatory interval series by the mean reaction time of the trials with 8 seconds preparatory interval in the irregular series. Scores above 1 are evidence of redundancy deficit. This method excludes interference from reaction time duration. In addition the Mean Reaction Time was computed as the mean reaction time duration of all series taken together.

\section{(c) Smooth Pursuit Eye Movements (Eye Tracking).}

Smooth pursuit eye movements were recorded electro-oculographically during a short term eye tracking task. Subjects were asked to wear corrective eyeglasses if needed. Testing was carried out in a sounddampened room. During eye movement recording subjects had their heada stabilized by chin and forehead rests, to minimize head movements. Subjects tracked a luminous spot of Light horizontally traversing 20 degrees of visual arc across an ascilloscope screen (Telequipment D 43). The target spot was driven sinusoidally at a frequency of 0.4 $\mathrm{Hz}$. The signal was derived from a wave generator. This procedure was preferred to digital wave form generation, because, unlike the digital procedure, it gives a perfectly smooth sine wave. Chin and forehead rests were attached to the oscilloscope to quarantee a fixed distance 
of approxinately $30 \mathrm{~cm}$ from nose bridge to sereen. To minimize distraction a viewing tunnel extended from the oscillascope screen to the head rests, with a flat back painted interior. The total duration of the experimental trial was nearly 41 seconds. Eye movements were measured by electro-oculography, silver-silverchloride electrodes being attached to the outer canthi of both eyes, and to the middle of the forehead as a ground reference. Both eye movement signal and ground aignal $(0.4 \mathrm{~Hz}$ ) were recorded on paper by an Elema-Schönander Mingograph (100 uW/cm). Time congtant was 1.2 seconds, upper frequency cut off filter $15 \mathrm{~Hz}$. The electro-oculographic signal was in addition analog-digital converted and recorded on magnetic tape for further analysis. The data were sampled with a frequency of $50 \mathrm{~Hz}$ during about 40 seconds (2048 data points).

The recording was started with a stationary target during about 40 seconds, which the subject was required to fixate. Next, immediately after a short instruction period to familiarize the subject with the nature of the tracking task, the eye tracking recording started with a pasitive zero crossing of the $0.4 \mathrm{~Hz}$ ground signal, corresponding with a leftuard movement of the spot on the oscilloscope screen. The timing of this zero crossing was done with a sampling frequency of $1000 \mathrm{~Hz}$.

Quantitative scoring was based on low frequency cut off filtering of the analog-digital converted standardized data. Filtering was done twice with a second ordier Butterworth filter (digitally), resulting in a fourth order Buttervorth filtering (computer: Heulett Packard 2100 5). By this procedure the $0.4 \mathrm{~Hz}$ sine wave disappeared and the 'noise' remained. For technical reasons the first and the last period of the sequence had to be excluded from the computations. These periods ere deformed by the computing program. 14 periods or 35 seconds of eye tracking were available for computation. Computed were the power of the filtered frequency data of each of these periods, as well as the mean power per period. In accardance with the recommendations of Lindsey, Holzman, Haberman and Yasillo, the natural logarsthm of the power score was used in the data analyses as a measure in agreement 
with qualitative evaluation of the power spectra. Included in the analyses are two SPEM scores: a Total score concerning the total 35 seconds of eye tracking, and a Best Cycle score, concerning the best period among the 14 constituting the total sequence.

\section{(d) Contingent Neqative Variation.}

CNV was recorded in a warned reaction time paradigm, with an interstimulus-interval of 3 seconds and a constant intertrial-interval of 17 seconds. This procedure was preferred to random intervals because prior experience indicated that patient subjects were more relaxed and not constantly preparing to a next trial. In addition there were less rejections because of movement artefacts than when using the random procedure. Both warning stimulus (SI) and imperative stimulus (S2) were auditory signals. 51 was a click ( $800 \mathrm{~Hz}, 50 \mathrm{msec}$, $55 \mathrm{~dB}), \mathrm{S2}$ was a tone ( $1000 \mathrm{~Hz}, 70 \mathrm{~dB})$, terminated by the response of the subject. The subject had to respond to $\mathbf{S 2}$ as quickly as possible by pressing a push-button with the thumb of his preferred hand. Subjects received a small number of instruction trials until they fully understood the procedure. The experiment was carried out in a sound-dampened, dimly lighted room. The subject was lying comfortably. A dim fixation light was mounted in front of the subject at a distance of about $1 \mathrm{~m}$. Silver-silverchloride electrodes were attached, according to the 10-20 system, with collodion to scalp sites $\mathrm{Fz}, \mathrm{Cz}$, $\mathrm{Pz}, \mathrm{C} 3$ and $\mathrm{C4}$. A reference electrode $\mathrm{Al}$ was attached to the left ear. $\mathrm{Fz}$ (frontal) and $\mathrm{Cz}$ (vertex) derivations were analysed in the frame of this investigation. To eliminate eye movement and blink artefacts, these vere recorded foto-electrically. Both signals - EEG and eye movement/blinking - were amplified by an Elema-5chönander Mingograph, with a time constant of 3.0 seconds and a high frequency cut of filter of $15 \mathrm{~Hz}$. (Although the built in gauging mechanism of the Elema-Schönander suggested a time constant of 9.0 seconds, the time constant proved to be only 3.0 seconds). Trials confounded by blinking or eye movements resulting in voltage deviations exceeding an empirically determined limit were automatically skipped. This limit was based upon an acceptable ratio between skipped and accepted 
trials. Triale in which a prenture reaction (before 52) occurred were also skipped. The experiment continued until 16 unconfounded trials were atored on magnetic tape. Signals were analog-digital converted, the data being sampled each $78.125 \mathrm{msec}$. A total of 256 data points was ampled for each trial (20.0 seconds), distributed in 110 points (about 6.5 seconds) immediatelly preceding SI, $3 B$ points ( 3.0 seconds) from 51 to 52 , and 108 points (about 8.5 seconds) following 52 . A11 computations, as well as the timing of the trials, were done by a Hewlett Packard $2100 S$ computer. The 16 trials were averaged and restored to a pseuda-DC recording. Further analyses were applied to these restored averages.

Pseudo-DC restoration as applied to the EEG data in this investigation is a technique developed by Mol and coworkers (Mol, Caberg and Rozendal, 1982). This technique results in the restoration of the averaged recording of a $R C$ amplifier to a vaveform pretty much the same as would have been recorded by a DC amplifier. The method consists in determining the transfer function of the EEG amplifier system, which is the division of the Fourier transform of the output signal by the fourier transform of the input signal. This procedure is most simple and phase problems are avoided when an input signal is used consisting of a block wave with exactly the duration of the sampling interval. Multiplication of the Fourier transform of any deformed output signal by the reciprocal of this transfer function restores the Fourier transform of the original input signal. The inversed Fourier transform of this frequency spectrum reconstructs the original signal. The reciprocal of the transfer function can thus be weed ao a filter to reatore any output signal of this particular amplifier systen to its initial shape, provided that time constant and sampling interval are held constant.

A computex program measured the amplitude of the CNV and of the post imperative negative potential. The baseline was defined as the average EEG of the first 109 data points of the recording (about $8.4 \mathrm{sec}$. preceding 51). Many investigators have based their baseline definition on a much shorter period preceding S1. A larger period can be expected 
to provide a mare reliable indication of the 'real' base level, as the level is well known ta fluctuate over time. In the arralysis the CNW was divided in an early and a late component. The amplitude of the early component was defined as the average EEG of data points 119-131 (covering a period of about $950 \mathrm{msec}$., starting about $625 \mathrm{msec}$ after 5I). The amplitude of the late component was defined as the average EEG of data points $136-148$ (covering about $950 \mathrm{msec}$, starting about 1950 msec. after S1). The PINV computation was based on the average EEG of 26 data points (about 1950 msec.), starting with data point 157 (625 msec. after S2). 

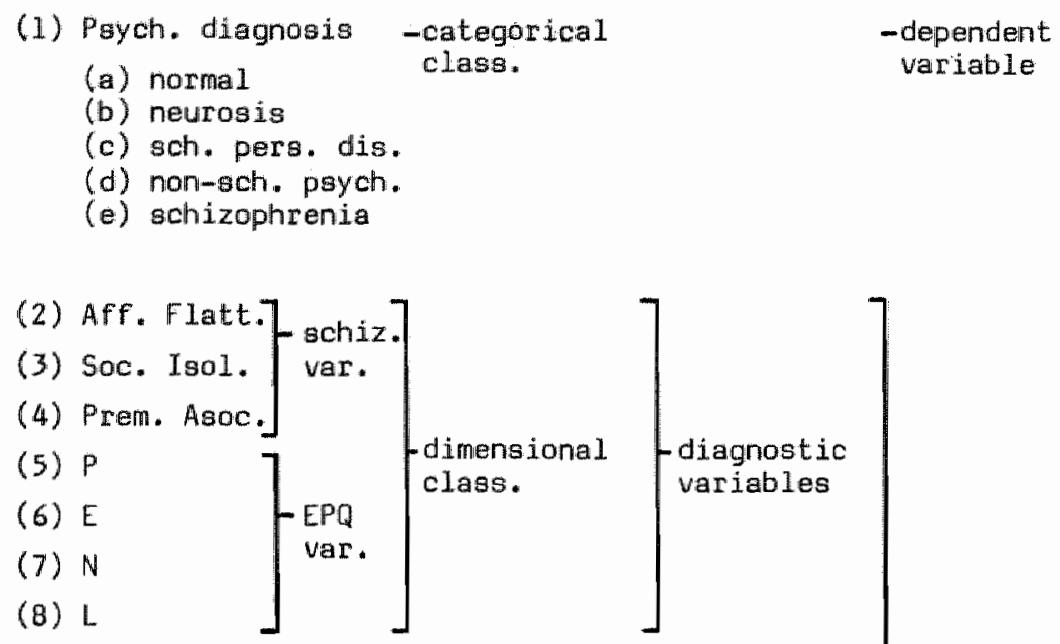

(9) SPEM Total

(10) SPEM Best Cycle

(11) Mean RT

(12) RT Red. Def.

(13) CPT Simple Om.

(14) CPT Compl. Om.

(15) CPT Simple Com.

(16) CPT Compl. Com.
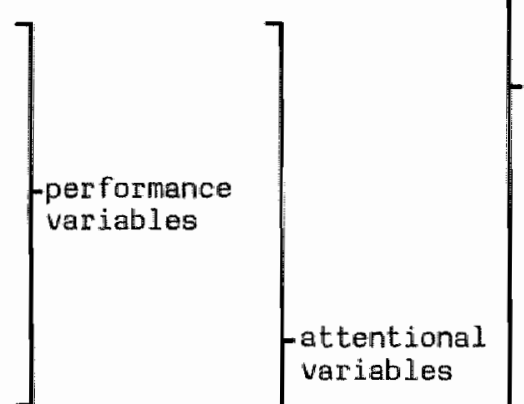

independent variables

(17) CNV Cz E.C.

(18) CNV Cz L.C.

(19) CNV Fz E.C.

(20) CNV Fz L.C.

(21) PINV Cz

(22) PINV FZ

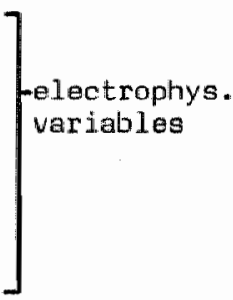

attentional variables
Hypothesis I : Intercorrelations variables (9) - (22).
Hypotheais II : Intercorrelations variables (1) - (4) (rank order diagnoses slightly modified, (c) and (d) changing places).

Hypothesis III: Correlation betweer, variables (1) and (5).

Hypothesis IV : Correlations between variables (1) and (9) - (22). 
Chapter 7

RESULTS

Spearman rank correlation coefficients were preferred in the correlational analyses, because these coefficients are 'distribution free' and may be used with scores that are ranks rather than numerical. This allows diagnoses to be introduced in the analyses as representing a rank order. When not indicated otherwise

(Hypothesis II!) peychiatric diagnoses are rank ordered in the analyses, according to severity, as follows: normal - neurosis schizotypal personality disorder - non-schizophrenic psychosis schizophrenia. 
7.1. IESTING QF HYPQTHESES.

7.1.1. Hypothesis I: intercorrelations of attentional variables.

'The electrophysiological and performance measures of attention in this investigation - Smooth Pursuit Eye Movements (Total and Best Cycle), Reaction Time (Mean and Redundancy Deficit), Continuous Performance Test (Simple and Complex Versions, Omission scores) and Contingent Negative Variation ( $\mathrm{Cz}$ and $\mathrm{Fz}$ derivations, early and late components, and Post Imperative Negative Variation) - are significantly positively intercorrelated'.

Correlational analyaeg.

To teat the validity of general deficit interpretations of attentional and information pracessing dysfunction in schizophrenia and related disorders intercorrelations were computed between all attentional variables in the total sample, and also in each group separately. In

Table 3. Spearman Correlation Coefficients.

\section{Total sample: Performance measures.}

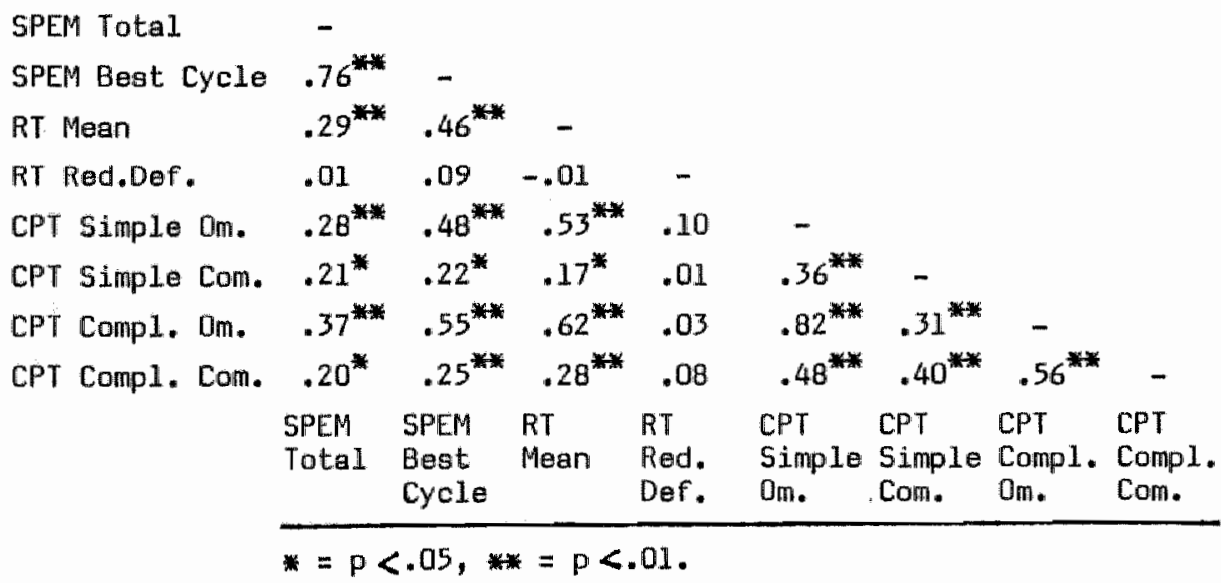


the total sample, comprising the subjects from all diagnostic groups, all correlations between performance variables are positive and significant at the 1 percent level, except for Reaction Time Redundancy Deficit measures: they totally lack significant correlations with any of the other performance measures (Table 3 ).

Table 4 presents the correlations between performance measures and electrophysiological measures of attention in the total sample. (In the calculations: the lower the CNV/PINV amplitude, the less negative or more positive, and consequently the higher the score!).

\section{Table 4. Spearman Correlation Coefficients.}

Total sample: Performance measures versus

Electrophysiological measures.

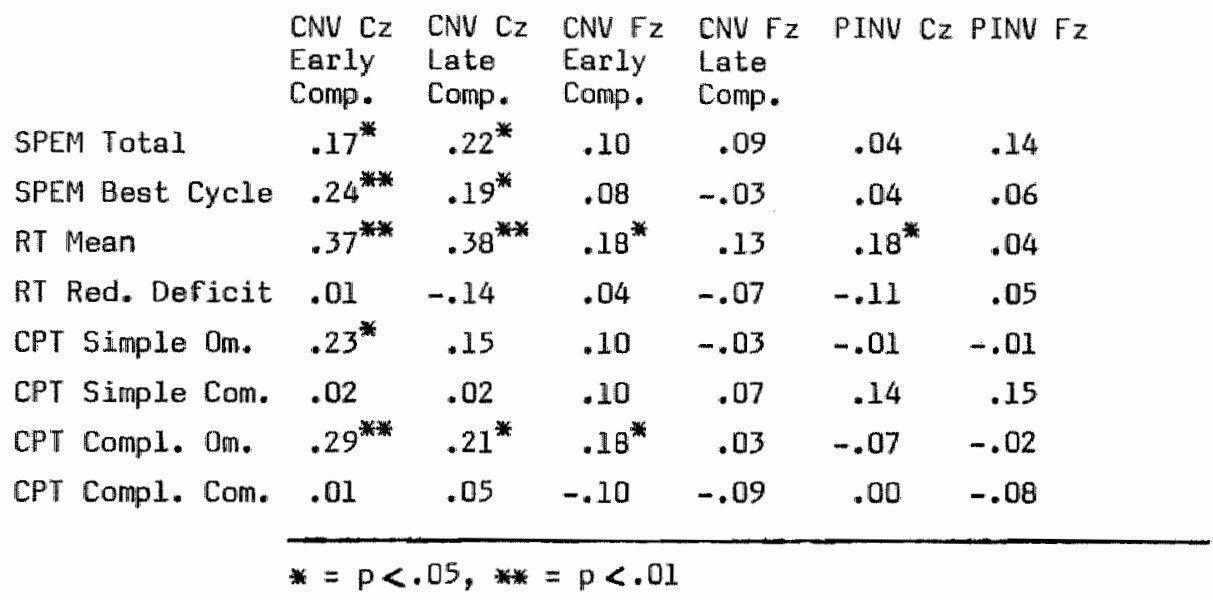

The correlations between SPEM measures and CNV measures appear to be significant $(p<.05)$ only for the central CNV derivations. The correlations in the total sample between Mean Reaction Time and CNV measures appear highest and indeed very significant $(p<.001)$ for the central CNV derivations too, but Reaction Time Redundancy Deficit does not correlate significantly with CNV measures. In the total ample CPT Omission scores correlate positively and significantly with central CNV's, but hardly with frontal CNV's. This finding again replicates 
the correlational pattern of the other performance variables. The correlations between performance measures and PINV measures are generally low and insignificant in the total sample. The direction of the few significant correlations is not as hypothesized. Negative correlations were postulated, some positive ones are found.

The intercorrelations between CNV and PINV measures in the total sample (Table 5) are all highly significant (p<.001). All correlations are positive, however, although negative correlations between CNV and PINV levels would have been in accordance with the relationship between both variables and (psychotic) disturbance of mental functioning.

Table 5. Spearman Correlation Coefficients.

Total sample: Electrophysiological measures.

CNV CZ Early Comp. -

CNV Cz Late Comp. $.75^{* *}$

CNV Fz Early Comp. $.74^{* *} .55^{* * *}-$

CNV Fz Late Comp. $.41^{* *} \quad .73^{* *} \quad .60^{* * *}$

PINV CZ

$.52^{* * *}$

$.55^{* * *}$

$.43^{* *}$

$.41^{* *}$

$.41 * *$

$.59^{* * *}$

$.38^{* *}$

$49^{* *}$

$.74^{* * *}$

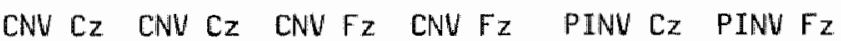

Early Late Early Late

Comp. Comp. Comp. Comp.

* $=p<.05, * *=p<.01$

Factor analysia.

To study in more detail the internal structure of the set of attentional variables factor analysis was applied to the scores of all subjects on the variables of this set (R-type factor analysis). The method preferred is principal factoring with iterations. The set of data is transformed into a new set of composite variables that are orthoganal (uncorrelated) to each other. Linear combinations of variables are formed, such, that the first principal component may be vieved as the best summary of linear relationship in the data. The second component is the second best linear combination of variables, under the condition that the second component is orthogonal to the first. It accounts for the most residual variance after the effects of 
the first component are removed from the data. This continues until all variance in the data is exhausted. To attain imple structure", varimax rotation can be applied to the factor matrix. This orthogonal rotational method produces the results in a form that makes more simple interpretation possible by accentuating the pealk processes in the derived factors.

Table 6 gives the varimax rotated factor matrix, shouing a total of 3 factors with an eigen value aboven 1 that account for 68.8 percent of the total variance. The six electrophysiological variables have the

Table 6. Principal Factor Analysis of Attentional Variables.

(CPT Conmission scores excluded).

Varimax Rotated Factor Matrix.

Factor I Factor II Factor III

$\begin{array}{lrrr}\text { SPEM Total } & .15 & .24 & .70 \\ \text { SPEM Best Cycle } & .09 & .37 & .92 \\ \text { Mean Reaction Time } & .22 & .62 & .17 \\ \text { Reaction Time Red. Def. } & -.04 & -.02 & .16 \\ \text { CPT Simple Omissions } & -.06 & .83 & .10 \\ \text { CPT Complex Omissions } & .03 & .94 & .11 \\ \text { CNU Cz Early Component } & .81 & .23 & .09 \\ \text { CNU Cz Late Component } & .79 & .28 & .07 \\ \text { CNU Fz Early Component } & .82 & .11 & -.05 \\ \text { CNV Fz Late Component } & .66 & .14 & -.08 \\ \text { PINV Cz } & .71 & -.19 & .02 \\ \text { PINV Fz } & .74 & -.18 & .04\end{array}$

Eigen value

Percentage

Cumulative

of variance

percentage

$\begin{array}{ll}\text { Factor I } & 4.18 \\ \text { Factor II } & 2.80 \\ \text { Factor III } & 1.28\end{array}$

34.8

34.8

23.3

58.2

10.6

68.6 
highest loadings - largely above .70 - on factor 1 . This factor is therefore interpreted as 'Contingent negative variation'. Factor II is characterized by high loadings - above .80 - on both CPT Omission measures, but also by a loading of more than .60 on Mean Reaction Time. This factor, being a composite of variables measuring speed and adequacy of reaction, can be labeled "response adequacy". Factor III has loadings above .70 on both SPEM measures, and thus represents "Smooth Purauit Eye Movementa". It is notable that Reaction Time Redundancy Deficit does not play a role in these factors. This is a confirmation of the outlier position of this variable as already demonstrated. When the CPT Commission measures take part in the factor analysis, the PINV measures emerge as a separate fourth factor, and the CPT Commission measures are included in factor II (Table 6a, Appendix 9). These resulta indicate that, as far as the selected attentional variables are rightly considered reliable indicators of the attentional deficit of psychosis and psychotic-like conditions, this deficit cannot be described as a unitary construct.

\section{Within-group analyses.}

Because the question arisis whether the degree of shared variance of the set of attentional variables might be dependent on the severity of psychiatric diagnosis, intercorrelations are exploratively studied within diagnostic groups as well. The intercorrelation matrices of the performance measures within the various diagnostic groups are shown in Tables 3a-3e (in Appendix 9). CPT Commission scores excluded, because they are considered not primerily related to attentional deficits, the number of positive intercorrelations $(p<.10)$ within diagnostic groups ranges from 3 (normals) to 13 (neuratics). These variables seem to share more common variance in non-psychotic patients (mean 11.5 significant positive correlations) than in psychotic patients (mean 5.5 significant positive correlations). When the intercorrelations between both SPEM measures and between both CPT Omission scores are also excluded, these figures are 9.5 and 3.5 respectively. Concerning the outlier position of Reaction Time Redundancy Deficit it is notevorthy that the neurotic group is an exception here, showing 
positive and significant correlations between Reaction Time Redundancy Deficit on the one hand, both SPEM measures and both CPT Omission scores on the other hand.

Analysis of correlations between performance and electrophyaiological measures within diagnostic groups (Tables 4a-4e, in Appendix 9) reveals that Reaction Time Redundancy Deficit, an outsider in the total sample, correlates significantly with CNV measures within the neurotic group. The carrelations between performance measures and PINV measures, insignificant or contrary to predictions in the tatal sample, are generally negative and partly significant in the schizotypic group. This category therefore conforms more or less to the predicted type of intercorrelations, while the other diagnostic categories do not.

The correlations between CNV and PINV measures within diagnostic groups show generally the same pattern as in the total sample, but some heterogeneity is apparent in the schizotypic group (Tables $5 a-5 e$, in Appendix 9).

\section{Conclusion.}

In the total sample the degree of positive intercorrelation within the set of performance variables appears reasonably high, although Reaction Time Redundancy Deficit is clearly out of tune with this set of variables. The positive intercorrelations of the variaus electrophysiological measures are fairly high, but the PINV"s do not behave as hypothesized. Only the correlational pattern found in the schizatypic group may tentatively be explained as suggesting a trend in the hypothesized direction. Concerning the wariance shared by performance and electrophysiological measures together is noteworthy that the positive correlations of central CNV's with performance measures show a tendency to be higher than those of frontal CNV's. Correlations between performance measures and PINV measuree are not as hypothesized. Only the schizotypic group shows some eignificant negative correlations. In sum, while the PINV's do not seem to share 
in the common variance, at least not in the predicted direction, Reaction Time Redundancy Deficit shows itself to be the real outsider in this company of attentional variables. The other variables appear generally positively intercorrelated to a reasonably high degree, but the principal factor analyais makes clear that in fact there are several dimensions within the set of all attentional variables. Although univariate analyses in the total sample give support to the first hypothesis, with the exclusion of the PINV measures and, especially Reaction Time Redundancy Deficit, multivariate analysis indicates the superficielity of this conclusion. The basic structure within the set of attentional variables is non-unitary, and characterized by three factors. Reaction Time Redundancy Deficit in the real outlier according to the factor analysis. Non-psychotic patients - schizotypics and neurotics - seem to share the highest degree of variance in attentional functioning, as operationalized by the set of performance variables. Psychotic - schizophrenic and non-schizophrenic - pretients show a smaller number of significant intercorrelations, but still more than normal controls. The intercorrelational pattern within the neurotic sample is particularly impressive. In this group nearly all relevant intercorrelations are statistically significant, even most of those invalving Reaction Time Redundancy Deficit, which otherwise has an outsider position. Reaction Time Redundancy Deficit is also significantly correlated with SPEM Best Cycle in the schizotypic group, and more correlations with other performance variables are positive in this group, but not to be a statistically significant degree. The association between Mean Reaction Time and the CPT Omission scores in all patient groups replicates the findings of Kornetsky and Drzack (1978). Pivik (1979) has noted a lack of association between simple reaction time and quality of eye tracking. The results in this investigation are varying acrose diagnostic categories, although in the total sample correlations are highly significant for SPEM Best Cycle, and somewhat legs significant for SPEM Total. There are some exceptions, but generally the selected performance variables appear to share a reasonably large degree of common variance. The higher number of signaficant positive intercorrelations in non-psychotic patients as 
compared with psychotic patients should probably be attributed to the more extreme scores and the higher within - subject variance in the latter category. However that may be, in this study intercorrelations between scores on performance variables appear highest in the intermediate range of clinically recognized psychiatric disturbance.

Analysis of the intercorrelations between the various CNV and PINV measures does not provide much information. CNV's and PINV's do not clearly emerge as separate phenomena in these analyses. (An exception is the factor analysis including CPT Commission scores). When the associations between electrophysiological variablea and performance variables are analysed, the difference between $\mathrm{CNV} \mathrm{Cz}$ and $\mathrm{CNV} \mathrm{Fz}$ measures is noteworthy. Only the CNV Cz measures show clear associations as predicted with SPEM measures, Mean Reaction Time and CPT Omission scores. The correlations between performance variables and PINV measures are not as predicted. An association between CNV amplitude and sustained attentional performance, probably comparable to the CPT, has also been reported by other investigators (Wilkinson and Seales, 1978). As discussed in Chapter 5.2.4, the relation between CNV and Reaction Time seems complicated, and overall the literature suggests that slow reaction time does not simply reflect itself in low CNV amplitude in within subject correlational analyses (Rebert and Tecce, 1973). Across subjects this type of association has been reported by some investigators (Naätanen and Gaillard, 1974; Papakostopoulos and Fenelon, 1975). This association is confirmed in this investigation, especially regarding the central CNV derivations.

\subsubsection{Hypotheses II and III: intercorrelations of diagnostic variables and psychiatric diagnosis.}

Hypothesis II: 'schizophrenia variables'.

The diagnostic measures Affective Flattening, Social Isalation, Premorbid Asociality, and psychiatric diagnosis, rank ordered: normal - neurosis - non-schizophrenic psychosis - schizotypal personality disorder - schizophrenia, are significantly positively 
The intercorrelations between the 'schizophrenia variables' Affective Flattening, Social Irolation and Premorbid Asociality, and psychiatric diagnosis rank ordered according to relationship with the schizophrenia concept, are presented in Table 7 for the total sample.

\section{Table 7. Spearman Correlation Coefficients.}

Total sample: 'Schizophrenia variables' and psychiatric diagnosis.

Diagnoses rank ordered: normal - neurosis - non-schizophrenic psychoois - achizatypal personality disorder - schizophrenia.

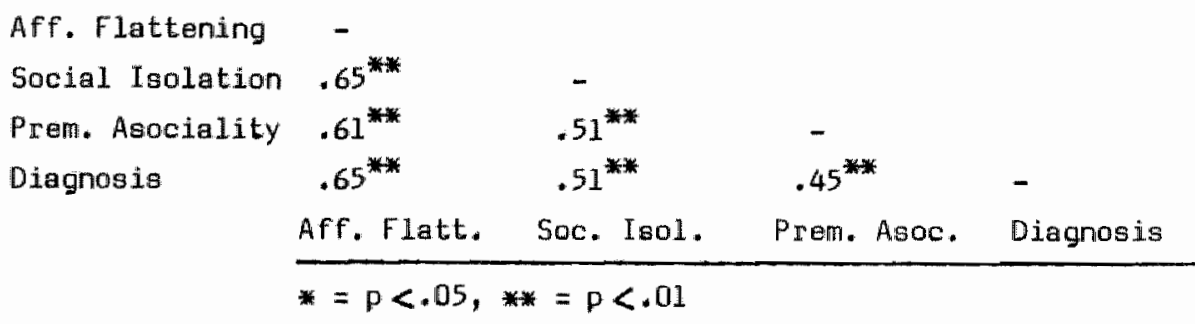

Affective Flattening, Sacial Isolation and Premorbid Asociality appear significantly positively intercorrelated, as well as significantly correlated with psychiatric diagnosis ranked as mentioned.

The mean scores and standard deviations on the 'schizophrenia variables", as well as the significance of difference between groups (Kruskal-Wallis one-way analysis of variance, non-parametric) are presented in Table B. Table 9 provides additional information on differences between groups. Figure 3 gives a visual impression of the differences between diagnostic groups. The relevance of Affective Flattening, Social Isolation and Premorbid Asociality to schizophrenia and schizotypia rather than to psychosis is general is clear. Kruskal-Wallis one-way ANOWA gives highest ranks concerning these diagnostic variables to the schizophrenic and schizotypic groups. 
These groups are not significantly different from each other. Third comes the non-schizophrenic psychotic group, but the difference with the neuratic group is not significant. Multivariate analyses will show, however, that Affective Flattening is far more important as a discriminator between diagnostic groups than both "social functioning" measures.

Table 8. Schizophrenia variables.

\begin{tabular}{|c|c|c|c|c|c|c|}
\hline & $\begin{array}{l}\text { Aff. } \\
\text { mean }\end{array}$ & $\begin{array}{l}\text { att. } \\
\text { S.D. }\end{array}$ & $\begin{array}{l}\text { Soc. } \\
\text { mean }\end{array}$ & $\begin{array}{l}\text { sol. } \\
\text { S.D. }\end{array}$ & $\begin{array}{l}\text { Prem. } \\
\text { mean }\end{array}$ & S.D. \\
\hline schizophrenia & 7.45 & 5.20 & 3.25 & 1.62 & 16.80 & 7.86 \\
\hline schizotypia & 7.25 & 5.32 & 3.45 & 1.57 & 15.10 & 8.61 \\
\hline non-schiz. ps. & 4.10 & 4.27 & 2.85 & 1.34 & 10.75 & 9.29 \\
\hline neurosis & 2.30 & 3.29 & 2.40 & 0.99 & 9.90 & 8.03 \\
\hline normal & 0.15 & 0.67 & 1.10 & 0.31 & 5.50 & 5.51 \\
\hline total & 4.25 & 4.12 & 2.61 & 1.38 & 11.61 & 7.97 \\
\hline $\begin{array}{l}\text { Chi-square } \\
\text { (corrected } \\
\text { for ties) }\end{array}$ & \multicolumn{2}{|c|}{45.27} & \multicolumn{2}{|c|}{33.79} & \multicolumn{2}{|c|}{$\begin{array}{l}21.13 \\
p<.001\end{array}$} \\
\hline
\end{tabular}

Table 9. Kruskal-Wallis one-way ANOWA: schizophrenia variables.

Significance of differences between diagnostic groups.

(Chi-square, corrected for ties). - = non-significant ( $p>$.jo)

$(1=$ normal, 2 = neurosis, 3 = schizotypal personality

disorder, 4 = non-schizophrenic prychosis, $5=$ actizophrenia $)$.

Aff. Flatt.

$\begin{array}{llllllllll}1 & 1 & 1 & 1 & 2 & 2 & 2 & 3 & 3 & 4\end{array}$ vs. vs. vs. va. vi. vs. va. va. ve. va.

Soc. Isol.

\begin{tabular}{llllllllll}
2 & 3 & 4 & 5 & 3 & 4 & 5 & 4 & 5 & 5 \\
\hline
\end{tabular}

Prem.Asoc.

$\begin{array}{llllllllll}.001 & .001 & .001 & .001 & .001 & - & .001 & .03 & - & .04 \\ .001 & .001 & .001 & .001 & .02 & - & - & - & - & - \\ .10 & .001 & .07 & .001 & .07 & - & .008 & - & - & .03\end{array}$




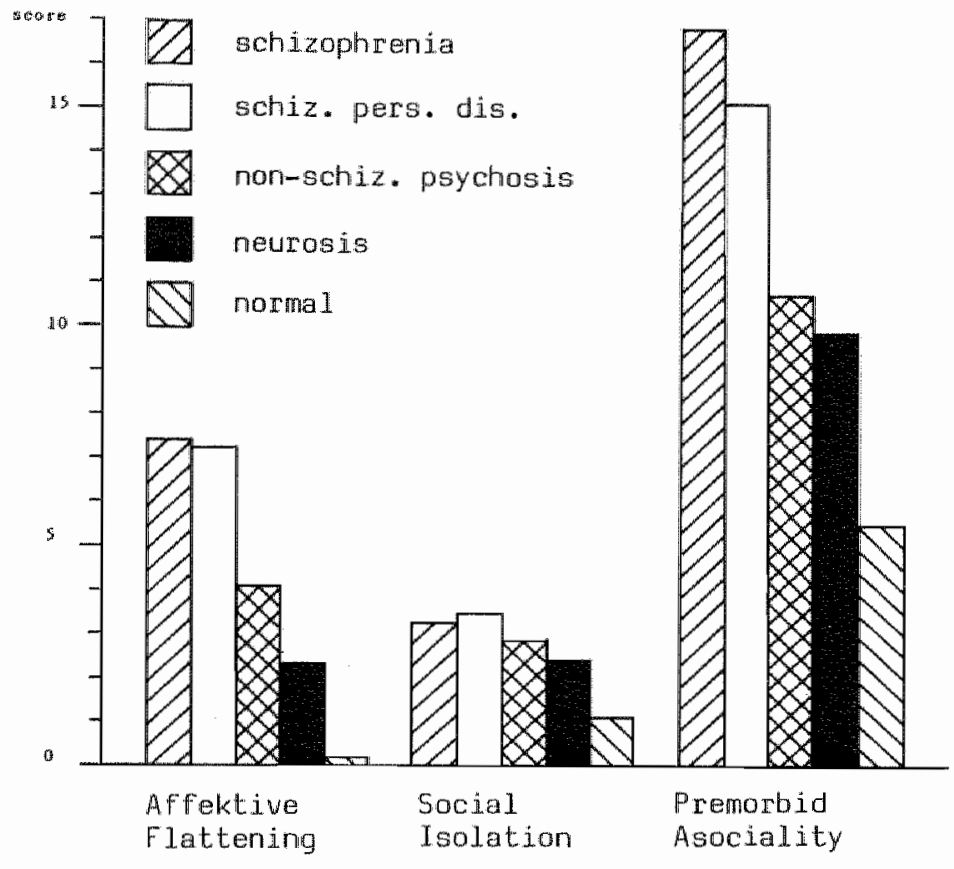

Hypothesis III: Psychoticism, and other EPQ measures.

'Psychoticism and severity of psychiatric diagnosis are significantly positively correlated. Diagnoses rank ordered: normal - neurosis schizotypal personality disorder - non-schizophrenic psychosis schizophrenia'.

The correlation between Psychoticism and severity of psychiatric diagnosis is included in Table 10. Psychoticism appears significantly $(p<.01)$ correlated with severity of psychiatric diagnosis. The more disturbed according to diagnosis, the higher the score on the Psychoticism scale. The absolute value of the correlation coefficient (.26) is, however, quite small. Figure 4 gives a visual impression of the differences between diagnostic groups concerning Psychoticism and the other scales of the Eysenck Personality Questionnaire. 


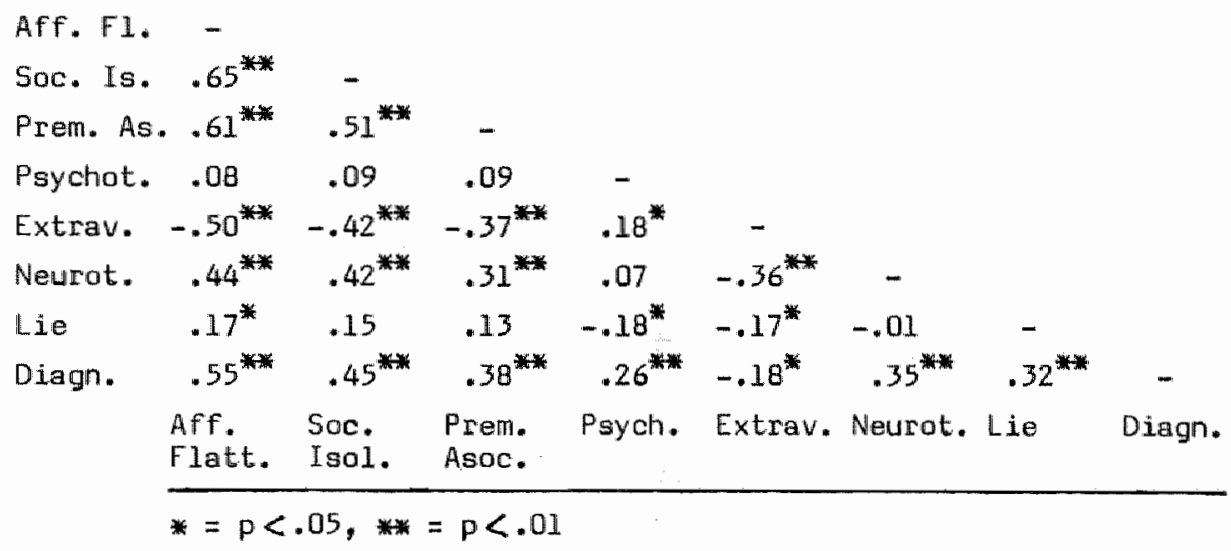

The other EPQ scales - Extraversion, Neuroticism and Lie - are included in the analyses because they too seem relevant the questions that are of interest here. Low degree of Extraversion is likely to denote aspects of personality that are also characteristic of Social Isolation; Neuroticism represents an alternative measure of psychopathology, besides Psychoticism; and Lie score, according to Eysenck and Eysenck (1975) should be taken into account in interpreting $P$ and $N$ scores. It measures dissimulation, but seems in addition to measure some stable personality factor which, according to the Eysenck's, "may possibly denote some degree of social naivete'. They stress, however, that little is known of this personality factor. Because too little is known at the moment about the nature of the $\mathrm{L}$ scale, no corrections are made in this investigation for differences along this dimension, but the scale participates exploratively in most of the statistical analyses.

The mean scores and standard deviations of the diagnostic groups on Psychoticism and on the other scales of the Eysenck Personality Questionnaire are provided in Table 11, together with the significance of difference between groups (Kruskal-wallis one-way analysis of variance). Table 12 gives additional information on differencer 


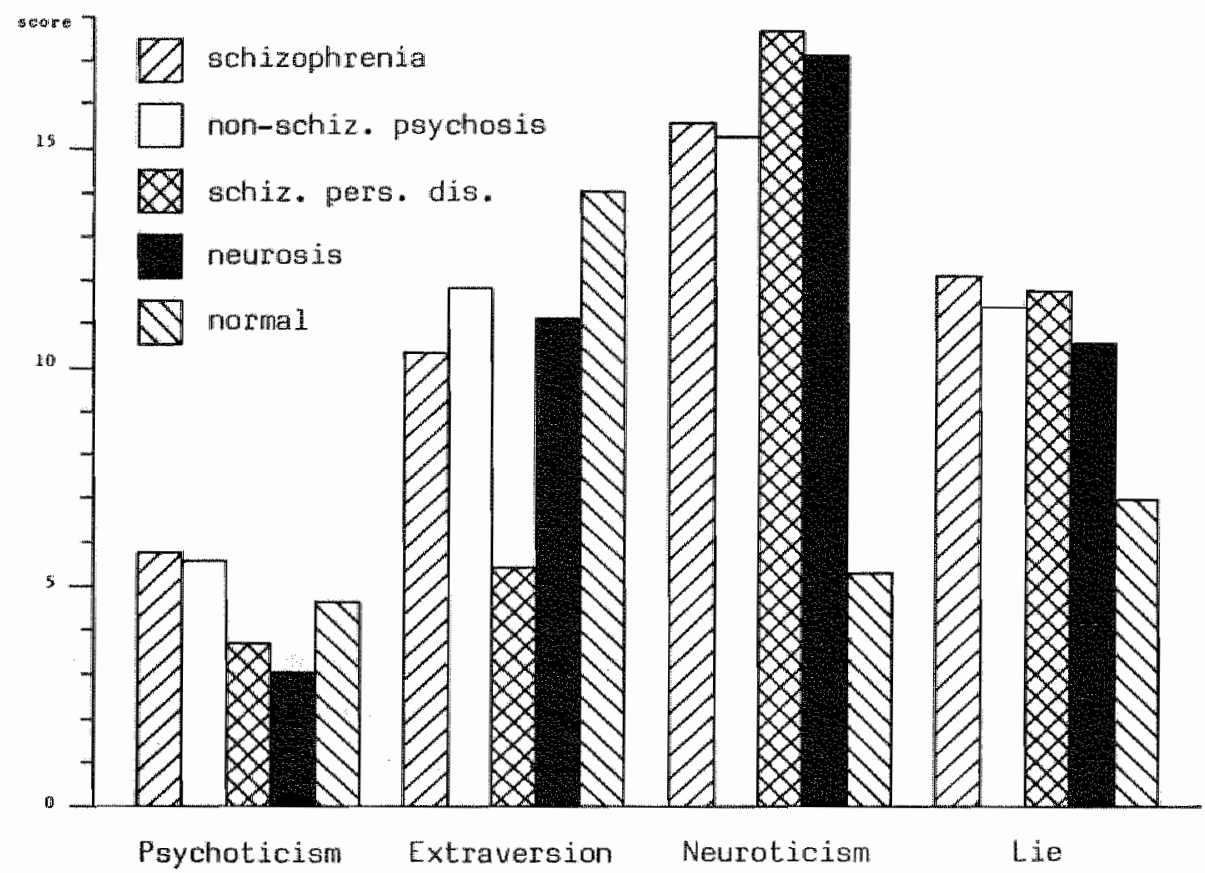

between groups. Psychoticism score ranks highest in both psychotic groups, which are not significantly different from each other. Significant differences are found between normals and neuratics, and evpecially between psychotic groups and non-psychotic patient groups. Extraversion scores appear lowest and significantly different from all other groups in the schizotypic group. Neuroticism scores are highest in bath non-psychotic patient groups, followed by the psychotic groups. None of the differences between patient groups reaches statistical. significance, however. The mean rank on Neuroticism of the normal group is extremely low, and significantly different from all patient. groups. A comparable pattern is found for Lie scores. Neuroticism and Lie scores are significantly positively correlated with rank ordered diagnoses, while Extraversion shows a less significant trend in the 
Table 11. Eysenck Personality Questionnaire scales.

\begin{tabular}{|c|c|c|c|c|c|c|c|c|}
\hline & \multicolumn{2}{|c|}{ Psychot. } & \multicolumn{2}{|c|}{ Extrav. } & \multicolumn{2}{|c|}{ Neurot. } & \multicolumn{2}{|l|}{ Lie } \\
\hline & mean & S.D. & mean & S.D. & mean & S.D. & mean & S.D. \\
\hline schizophrenia & 5.80 & 3.09 & 10.40 & 5.38 & 15.70 & 5.38 & 12.15 & 4.75 \\
\hline non-schiz. ps. & 5.60 & 2.37 & 11.90 & 4.38 & 15.40 & 6.26 & 11.50 & 5.06 \\
\hline schizotypia & 3.70 & 2.15 & 5.50 & 4.21 & 17.80 & 2.98 & 11.85 & 4.68 \\
\hline neurosis & 3.00 & 1.86 & 11.20 & 4.96 & 17.25 & 4.19 & 10.65 & 4.65 \\
\hline normal & 4.65 & 2.76 & 14.10 & 4.06 & 5.35 & 3.82 & 7.05 & 4.97 \\
\hline total & 4.55 & 2.49 & 10.62 & 4.63 & 14.30 & 4.67 & 0.67 & 4.82 \\
\hline hi-square & 16. & & 27.54 & & 39.08 & & 13.03 & \\
\hline & $\mathrm{P}<$ & & $\mathrm{P}<$. & & $\mathrm{P}<\mathrm{.O}$ & & $\mathrm{P}<.01$ & \\
\hline
\end{tabular}

Iable 12. Kruskal-Wallis one-way ANOVA: EPQ variables.

Significance of differences between diagnostic groups. (Chi-square, corrected for ties).

- = non-significant $(p>\cdot 10) \cdot(1=$ normal, $2=$ neurosis,

3 = schizotypal personality disorder, $4=$ non-schizophrenic psychosis, 5 = schizophrenia).

Psychoticism

Extraversion

Neuroticism

Lie

\begin{tabular}{cccccccccc}
1 & 1 & 1 & 1 & 2 & 2 & 2 & 3 & 3 & 4 \\
vs. & vs. & vs. & va. & vs. & vs. & vs. & vs. & vs. & vs. \\
2 & 3 & 4 & 5 & 3 & 4 & 5 & 4 & 5 & 5 \\
\hline .07 & - & - & - & - & .001 & .002 & .02 & .02 & - \\
.06 & .001 & - & .03 & .001 & - & - & .001 & .005 & - \\
.001 & .001 & .001 & .001 & - & - & - & - & - & - \\
.03 & .005 & .02 & .002 & - & - & - & - & - & -
\end{tabular}


Intercorrelations of 'schizophrenia variables' and EPQ variables.

Table 10 shows that in the total sample Psychoticism is not related to the three 'schizophrenia variables' - Affective Flattening, Social Isolation, and Premorbid Asociality. Not unexpectedly, the intercorrelations between Extraversion score and these variables are negative and highly significant, while the correlations between Neuroticism score and these variables are positive and highly significant. In the total sample Neuroticism is independent af Psychoticism, but several correlations between EPQ scales are significant. Extraversion is positively correlated with Psychoticism, and negatively with Neuroticism. Lie scores correlate negatively and significantly with Psychoticism as well as Extraversion, but not with Neuroticism. These intercorrelations are on the whole comparable with those presented by Eysenck and Eysenck (1975). They considered their statistically significant but quite low correlations not serious from an applied point of view. The highest correlation between EPQ scales found in the present study is between Extraversion and Neuroticism $(-.36, p<.001)$, which accords with the pattern Eysenck and Eysenck found in abnormal (patient and criminal) groups.

\section{Factor analysis.}

Principal factor analysis of the set of diagnostic variables (psychiatric diagnosis excluded) shows 2 factors with an eigen value above 1 , accounting for 65.4 percent of the total variance (Table 13). In the varimax rotated factor matrix loadings above .50 on factor I are found on the variables Affective Flattening, Social Isolation, Premarbid Asociality, Extraversion (inversed sign), and Neuroticism. This factor seems to represent 'general maladaptive psychosacial functioning'. Factor II is characterized by a relatively high loading on Extraversion (note the sign reversal between both factors), and a moderately high, but supposedly meaningful, loading on Psychoticism. When the Lie scores are included in the analysis, Psychoticism receives a loading of .75 on factor II, which factor is further 
characterized by probably to some degree meaningful loadings of about .30 on Extraversion and Lie (inversed sign). Tentatively, this second factor is labeled 'acting out'. The independence of Psychoticism from the other diagnostic variables is thus most clearly demonstrated by factor analysis including Lie scores (Table 13a, in Appendix 9), which confirms Eysenck's notion that in evaluating the meaning of the $P$ score the $\mathrm{L}$ score should be taken into account.

Table 13. Principal Factor Analysis of Diagnostic Variables. (Lie scores excluded).

Varimax Rotated Factor Matrix.

Factor I Factor II

$\begin{array}{lrr}\text { Affective Flattening } & .81 & .13 \\ \text { Social Isolation } & .75 & .12 \\ \text { Premorbid Asociality } & .66 & .18 \\ \text { Psychaticism } & .10 & .36 \\ \text { Extraversion } & -.68 & .57 \\ \text { Neuroticism } & .51 & -.06\end{array}$

Eigen value

Factor I

Factor II

\subsection{2}

1.10

\section{Percentage} of variance
Cumulative percentage

$\begin{array}{ll}47.1 & 47.1 \\ 18.4 & 65.4\end{array}$

Mutiple regression analysis.

Multiple regression analysis offers the opportunity to analyse the relationship between a dependent (criterion) variable and a set of independent (predictor) variables. The overall dependence of severity of psychiatric diagnosis - rank ordered: normal - neurosis schizotypal personality disorder - non-schizophrenic psychosis schizophrenia - on the sets of diagnostic and attentional variables can thus be studied. Another application of this method is the use of partial regression coefficients as measures of the influence of each independent variable taken individually while controlling for the influence of all other independent variables. Stepwise multiple 
regreasion analysis determines the predictive power of increasingly larger subsets of independent variables. Optimum predictive combinations of variables are selected, and the procedure stops at the level beyong which addition of further variables does not improve the prediction.

The results of stepuise multiple regression analysis of the diagnostic variables are presented in Table 14. In the table, $R$ is the multiple regresion coefficient and $\mathrm{R}^{2}$ is the explained variance at the selected level (step). In addition, the standardized partial regression coefficients are presented. Table 14 shows that the set of diagnostic variables explains 44.4 percent of the tatal variance.

Table 14. Stepuise Multiple Regression Analysis.

Diagnostic variablas.

criterion variable: rank ordered psychiatric diagnoses.

\begin{tabular}{lllll}
\hline Step No. Diagnostic variable & $\mathbb{R}$ & $R^{2}$ & $\begin{array}{l}\text { Standardized partial } \\
\text { Regression Coeff. }\end{array}$ \\
\hline 1 & Affective Flattening & .473 & .223 & .253 \\
2 & Neuroticism & .540 & .291 & .278 \\
3 & Lie & .592 & .350 & .313 \\
4 & Psychoticism & .650 & .423 & .242 \\
5 & Extraversion & .658 & .433 & .140 \\
6 & Social Isolation & .664 & .441 & .109 \\
7 & Premorbid Asociality & .667 & .444 & .075
\end{tabular}

The predictive capacity of the diagnostic set is largely due to the first four selected variables. The 'social functioning' variables hardly add to the explained variance attained by Affective Flattening, Neuroticism, Lie and Paychoticism. Although Lie is not really a diagnostic measure, it ranks among the best predictors and shows even the higheet partial regression coefficient. Table 14 shows that the best predictors among the set of diagnostic variables are also the varjables with the lhighest partial regression coefficients. 
Within-group correlations uere studied in order to correct for possible confounding influences of psychiatric diagnosis as a moderator variable. The positive intercorrelations, with various degrees of signaficance, between Affective Flattening, Social. Isolation and Premorbid Asociality within the diagnostic groups indicate that the shared variance of these 'schizophrenia variableg' is independent of the diagnosis of schizophrenia (Tables 10a-10e, in Appendix 9). The within-group correlational analyses of the EPQ measures reveal significant correlations between Paychoticism and Neuroticism in both psychotic groups, contrasting with the results of Eysenck and Eysenck (1975). Most correlations are non-significant.

\section{Discriminant analyses.}

The danger of getting a distorted picture of group differences tends to increase as the correlations among the variables become larger, although this distortion is not directly related to these correlations. Differences between groups on some variable nay be merely the result of an association between this variable and another variable, which has an even higher discriminating power. As the number of variables increases, the difficulty of interpreting differences between groups on each variable taken singly becomes more serious. An alternative way to describe group differences is discriminant analysis: the construction of a linear combination (a weighted sum) of the set of variables that will maximally differentiate among the groups in question. By this technique variables are weighted and combined in some fastion so that the groups, defined in advance, are forced to be as statistically different as posible. The weighting coefficients serve to identify the variables which contribute most to differentiation along the respective dimension (function), while their sign indicates in which direction they contribute. Contribution in positive direction is contribution descriptive of the group having the higher mean scare on this linear combination, while negative direction is descriptive of the group with the lower mean score. Examination of the pattern of weights gives a much more accurate account of the nature of group differences in terms of a given set of variablea than does looking at each variable separately, with no regard for their interrelations and partly overlapping information (Tatsuaka, 1970). In this investigation a stepwise procedure uas preferred. The stepwise procedure begins by selecting the single best-discriminating variable according to some criterion (here: the minimum Mahalanobis distance between the two closest groups). Next, a second discriminating variable is selected as the variable best able to improve the value of 
the discriminating criturion in combination ith the first variable. Third and subsequent variables are similarly selected according to their ability to contribute to further discrimination. At each step, variables already elected mey be removed if they are found to reduce discrimination when combined with more recently selected variables. Eventually, either all variables will have been selected, or it will be found that the remaining variables are no longer able to contribute to further discrimination. The stepuise procedure than halts.

Interest focussed on the questions which diagnostic variables are the most important ones for the discrimination of:

(a) patients belonging to the schizophrenic, non-schizophrenic psychotic and schizotypic groups (schizophrenia-like conditions) vergus neurotic patients and normals,

(b) psychotic (schizophrenic and non-schizaphrenic) patients versus non-psychotic (schizotypic and neurotic) patients and normals,

(c) psychotic patients versus nom-psychotic patients,

(d) more disturbed patients, whether psychatic or not, versus less disturbed patients or normals (stepwise, across the whole range of rank ordered psychiatric diagnoses: schizophrenia - nonschizophrenic psychosis - schizotypal personality disorder neurosis - normal).

Psychoticism and Neuroticism appear to be the most important variables in the function of psychotics versus non-psychotic patients (Table 15). When diagnostic groups are compared singly instead of in combinations, Psychaticism is present in most of the discriminant functions. Paychoticism does not appear in the discriminant function of all patient groups versus normals. Here the highest loading is on Neuroticism, tuice as large as the next highest loading variable, Lie. Overall, Social Igolation and Premorbid Asociality seem relatively unimportant as discriminating variables. The general high ranking of Psychoticism in discriminations between patient groups is noteworthy, as is the fact its sign consistently points in the direction of the more disturbed diagnostic category. No consistency in this respect is evidient in the coefficients of Extraversion and Neuroticism. Affective 
Flattening behaves Iike Psychoticism and is also in all functions indicative of the higher level of psychiatric disturbance as clinically recognized.

\section{Table 15. Stepuise discriminant analysis:}

\section{diagnostic variables.}

Groups: (neuratics + schizotypics) varsus (non-schizophrenic psychotics + schizophrenics).

$\begin{array}{llll}\text { Step } & \text { Variable } & \text { Rao's } V & \text { Change in } \\ \text { number } & \text { entered } & & \begin{array}{l}\text { Sign of of } \\ \text { change }\end{array}\end{array}$

\begin{tabular}{lllcl}
\hline 1 & Psychoticism & 19.25 & 19.25 & .000 \\
2 & Neuroticism & 30.26 & 11.01 & .001 \\
3 & Extraversion & 32.50 & 2.24 & .14 \\
4 & Aff. Flatt. & 35.13 & 2.64 & .10 \\
\hline
\end{tabular}

Standardized discriminant function coefficients:

$\begin{array}{lr}\text { Psychoticism } & .78 \\ \text { Neuroticism } & -.44 \\ \text { Extraversion } & .35 \\ \text { Affective Flattening } & .26\end{array}$

Correctly classified: $72.5 \%$

(In the Discriminant Function tables a negative sign points in the direction of the category mentioned first, and a poeitive sign points in the direction of the second category. The first category is always the clinically less disturbed category, so that a negative eign points in the direction of the psychiatrically less disturbed categary, and a positive sign in the direction of the more disturbed category. The rank order, from low to high psychiatric disturbance, is: normal - neurosis - schizatypal personality disorder non-schizophrenic psychosis - schizaphrenia). 
Iable 16. Stepwise discriminant analysis: diagnostic variables.

Groups: (normals + neurotics) versus (schizotypics + non-schizophrenic psychotics + schizophrenics).

\begin{tabular}{lllll}
$\begin{array}{l}\text { Step } \\
\text { number }\end{array}$ & $\begin{array}{l}\text { Variable } \\
\text { entered }\end{array}$ & Rao's $V$ & $\begin{array}{l}\text { Change in } \\
\text { Rao's } V\end{array}$ & $\begin{array}{l}\text { Sign. of } \\
\text { change }\end{array}$ \\
\hline 1 & Aff. Flatt. & 33.24 & 33.24 & .000 \\
2 & Lie & 42.18 & 8.94 & .003 \\
3 & Psychoticiam & 52.94 & 10.76 & .001 \\
4 & Neuroticiom & 63.90 & 10.96 & .001 \\
5 & Soc. Isol. & 66.21 & 2.31 & .13 \\
\hline
\end{tabular}

Standardized discriminant function coefficients:

Affective Flattening $\quad .45$

Lie $\quad .43$

Psychoticism $\quad .35$

Neuroticism .31

Social Isolation $\quad .19$

Correctly classified: $79 \%$ (Negative sign points to first category)

The questions $(a)-(d)$ can be answered as follows:

(a) The best discriminating diagnostic veriable between patients showing schizophrenia - like conditions and other (neurotic) patients and normals is Affective Flattening (Table 16).

(b) The best discriminating diagnostic variable between psychotic patients and non-psychotic patients plus normals is Psychoticism (Table 17).

(c) The best discriminating diagnostic variable between psychotic and non-psychotic patients is Psychoticism (Table 15) 
(d) The best discriminating diagnostic variable between more and less disturbed patients or normals appears to be Psychoticism then the patient groups are considered. Neuroticism is the best diagnostic discriminator between normals and patient groups, whether psychotic or not.

Table 17. Stepuise discriminant analysis: diagnostic variables.

Groups: (normals + neurotics + schizotypics) versus (non-schizophrenic psychotics + schizophrenics).

\begin{tabular}{lllll}
$\begin{array}{l}\text { Step } \\
\text { number }\end{array}$ & $\begin{array}{l}\text { Variable } \\
\text { entered }\end{array}$ & Rao's $V$ & $\begin{array}{l}\text { Change in } \\
\text { Rao's } V\end{array}$ & $\begin{array}{l}\text { Sign. of } \\
\text { change }\end{array}$ \\
\hline 1 & Psychoticism & 14.06 & 14.06 & .000 \\
2 & Lie & 24.72 & 10.67 & .001 \\
3 & Aff. Flatt. & 29.14 & 4.42 & .04 \\
4 & Extraversion & 34.19 & 5.05 & .03 \\
5 & Neuroticism & 36.77 & 2.58 & .11 \\
\hline
\end{tabular}

Standardized discriminant function coefficients:

$\begin{array}{ll}\text { Psychaticism } & .65 \\ \text { Lie } & .50 \\ \text { Affective Flattening } & .47 \\ \text { Extraversion } & .47 \\ \text { Neuroticism } & .25\end{array}$

Correctly classified: $72 \%$ (Negative sign points to first category)

\section{Influence of sex and aqe.}

The influence of sex and age on the 'schizophrenia variables" Affective Flattening, Social Isolation and Premorbid Asociality seems limited. The only significant (positive) correlation is between Social Isolation and age $(p<.05)$, which is not surpriaing in vieu of 
the well-known decline of social involvement with increasing age. There are no significant correlations between Psychoticism score and sex or age. Neuroticism score is higher in umen than in men $(p<.10)$. This is a vell-known finding. Women score also higher on the Lie scale $(p<.01$ ) (but the balance between men and women has shifted in favor of the latter in the psychotic groups, so diagnosis may serve as a moderator variable here), and there is an even higher correlation between Lie score and age $(p<, 001)$. None of the remaining correlations between EPQ scores and age or sex reaches statistical significance.

Kruskal-Wallis one-way ANOVA's of the subpopulations of younger and older subjects (split across the median, categories 20-32 and 33-59 years of age) show still aignificant differences between groups for Social Isolation in both subpopulations $(p<.01)$. When men and women are studied separately, the difference between groups for Neuroticism appears also significant $(p<.01)$ in both. Lie scores, however, appear not significantly different between groups in older subjects and in women.

\section{Conclusion.}

Both the second and the third hypothesis are supported by the results. Affective Flattening, Social Isolation, Premorbid Asociality and psychiatric diagnosis, rank ordered according to relationship with the schizophrenia concept, are significantly positively intercorrelated. Psychoticism is significantly positively correlated with severity of poychiatric diagnosis.

The diagnostic variables Affective Flattening, Social Isolation and Premorbid Asociality have demonstrated their relevance to the diagnoses of schizophrenia and schizotypal personality disorder, more than to the other diagnostic categories in this study. The non-schizophrenic psychotic category in this investigation appears distinct from the two diagnostic categories just mentioned, so far as these 'schizophrenia variables" are concerned, although oniy half of 
the differences between these groups are significant. Differences with normals are significant for these variables in all patient groups. The relatively high degree of positive intercorrelation of these three variables is not unexpected, as their respective definitions (sets of criteria) already suggested some degree of homogeneity. The fact that these interrelationships apply to all diagnostic groups implies that they cannot be attributed to schizophrenia or psychosis as a moderator variable. The association between 'schizophrenia variables' and psychiatric diagnoses are of course biased in favour of the 'schizophrenia-like' conditions by their partial inclusion in the (polythetic) sets of criteria for schtzophrenia and schizotypal personality disorder according to DSM-III. Besides, diagnosis and 'schizophrenia variables' were not assessed by independent investigators. Affective Flattening appears far more important as an indicator of severity of disturbance than the other 'schizophrenia variables', Social Isolation and Premorbid Asociality. Affective Flattening is the highest ranking diagnostic variable in the stepuise multiple regression analysis, and it is consistently indicative of the more disturbed category in the discriminant analyses.

The EPQ scale considered most interesting, the Psychoticism acale, demonstrates its association with psychosis by receiving significantly higher scores in psychotics than in non-psychotic patients. The multivariate analyses confirm the importance of this diagnostic measure as an indicator of severity of psychiatric disturbance. But its power as a discriminator diminishes when normals are included in the sample. This suggests that its power may be classifying patient populations rather than mixed - patient plus normal - populations. It is not evident how to explain this finding from the postulation of Psychoticism as a basic personality dimension related to the predisposition to develop a psychosis. In any case, within a psychiatric patient sample its association with psychosis has been demonstrated. It should be noted that within the patient sample differences between diagnostic groups concerning Lie scores are not statistically significant. Eysenck's warning that Lie scores should be taken into account when considering Psychoticism scores (confirmed in 
this study by the results of factor analysis) does not seem to apply to the interpretation of differences between patient groups in this investigation. It might add to the understanding of the relatively high $P$ cores in the normal sample, which shows a significantly lower Lie score than the patient groups. These Lie scores enhance the contrast between non-psychotic patient and normal groups concerning Psychoticism scores, and obscure the differences along the poychaticiam dimension between psychotic patients and normals.

Psychoticism seems not associated with Affective Flattening, Social Isolation or Premorbid Asociality, so Psychoticism and the set of these 'schizophrenia variables' are to be considered relatively independent 'dimensions', as is shown by factor analysis of the diagnostic variables, especially when the EPQ Lie scores are included. This factor analysis generates two factors: a first factor comprising Affective Flattening, Social Isolation, Premorbid Asociality, Neuroticism and Extraversion (reversed sign), and a second factor consisting of Psychoticism. Thus, after the dimension of igeneral maladaptive psychosocial functioning' has been eliminated, Psychoticism appears. The difference between psychatic (schizophrenic and non-schizophrenic) patients and schizotypic patients, with respect to diagnostic variables, is mainly determined by Psychoticism and Extraversion. Psychotics score higher on both variables (the 'acting out" factor of the factor analysia).

In contrast to Psychoticism, Neuroticiam scores are highest in non-psychotic patients - schizotypics and neurotics -, although the differences with psychotics are not statistically significant. The independence of both personality dimensions, as postulated by Eysenck, is not demonstrated within the psychotic groups. The findings suggest that Neuroticiam is a general indicator of maladaptive stressful functioning, while Psychoticism is a more specific indicator within a psychiatric patient population of "psychotiform" disturbance. 
The results with respect to the Lie scale suggest that this measure is also of some importance as an indicator of psychotic disturbance and, generally, of severity of psychiatric disturbance. Its importance seems not 1 imited to its role in interpreting $P$ and $N$ scores.

The major canclusions seem the following:

The results confirm the notion that schizophrenics and schizotypics, as defined by DSM-III, are more closely related with each other than with non-schizophrenic psychotic (DSM-III) subjects at the level of affective and social functioning. Psychoticism, as defined by the Eysenck Personality Questionnaire, has no special relevance to the schizophrenia concept inherent in DSM-III, but it does shou relevance to the DSM-III concept of non-affective functional psychosis in general.

7.1.3. Hypothegis IV: correlations between attentional variables and psychiatric diagnosis.

"Degree of irregularity of Smooth Pursuit Eye Movements, duration of Mean Reaction Time, degree of Reaction Time Redundancy Deficit, percentage of omissions on the Continuous Performance Test, degree of depression of the Contingent Negative Variation, and level of Post Imperative Negative Variation are significantly positively correlated with severity of psychiatric diagnosis. Diagnoses rank ordered: normal - neurosis - schizotypal personality disorder non-schizophrenic psychosis - schizophrenia".

This is the central hypothesis of this investigation. As shown by Table 18 the highest correlations with severity of diagnosis are found for the attentional variables SPEM Best Cycle, Mean Reaction Time, CPT Simple Version Omission score, CPT Complex Version Omission score, and CNV Cz Early Component - all highly significant $(\mathrm{P}<.001$ ). Also significantly correlated with severity of diagnosis are CNV Cz Late Component $(p<.01)$ and both CPT Commission scores $(p<.05$ and $<.001)$. The correlation of severity of diagnosis with SPEM Total is marginally significant $(p<.10)$, and the correlations with Reaction Time 
Redundancy Deficit, both CNV Fz measures and both PINU measures are clearly insignificant.

Table 18. Spearman correlation coefficients.

Attentional variablee versus severity of psychiatric diagnosig.

Severity of Diagnosis

$\begin{array}{lc}\text { SPEM Total } & .13 \\ \text { SPEM Best Cycle } & .35^{* *} \\ \text { RT Mean } & .43^{* *} \\ \text { RT Redundancy Deficit } & -.07 \\ \text { CPT Simple Dmissions } & .51^{* *} \\ \text { CPT Simple Commissions } & .17^{*} \\ \text { CPT Complex Omissions } & .48^{* *} \\ \text { CPT Complex Commissions } & .32^{* *} \\ \text { CNV Cz Early Component } & .31^{* *} \\ \text { CNV Cz Late Component } & .25^{* *} \\ \text { CNV Fz Early Component } & .05 \\ \text { CNV Fz Late Component } & -.03 \\ \text { PINV Cz } & .07 \\ \text { PINV Fz } & -.04\end{array}$

$*=p<.05, * *=p<.01$

The mean scores and standard deviations on the attentional measures, with the significence of differences, between groups (Kruskal-Wallis one-way analysis of variance), are presented in Tables 19-22. Table 23 provides additional information on differences between groups. Figures 5-8 give a visual impression of the differences between diagnostic groups. The attentional variables that differentiate best between the diagnostic groups are SPEM Best Cycle, Mean Reaction Time, CPT Simple Version Omission score, CPT Complex Version Omission score $(p<.001)$, and CPT Complex Version Commission score $(p<.003)$. All are performance measures. Significant differences for SPEM Best Cycle are found between 
Iable 19. Smooth Pursuit Eye Movements scores.

\begin{tabular}{|c|c|c|c|c|}
\hline & \multicolumn{2}{|c|}{$\begin{array}{l}\text { SPEM Total } \\
\text { (In-piower) }\end{array}$} & \multicolumn{2}{|c|}{$\begin{array}{l}\text { SPEM Best Cycle } \\
\text { (In-power) }\end{array}$} \\
\hline & mean & 5.0. & mean & S.D. \\
\hline schizophrenia & 3.49 & 0.99 & 2.27 & 1.25 \\
\hline non-schiz. ps. & 3.78 & 1.30 & 2.59 & 1.03 \\
\hline schizotypia & 3.45 & 1.09 & 1.91 & 0.93 \\
\hline neurosis & 3.70 & 1.34 & 2.06 & 1.22 \\
\hline normal & 2.99 & 1.06 & 1.21 & 0.72 \\
\hline total & 3.48 & 1.16 & 2.01 & 1.05 \\
\hline $\begin{array}{l}\text { Chi-square } \\
\text { (corrected } \\
\text { for ties) }\end{array}$ & $\begin{array}{l}6.50 \\
p<.1\end{array}$ & & $\begin{array}{l}19.2 \\
\rho<.\end{array}$ & \\
\hline
\end{tabular}

Table 20. Reaction Time scares.

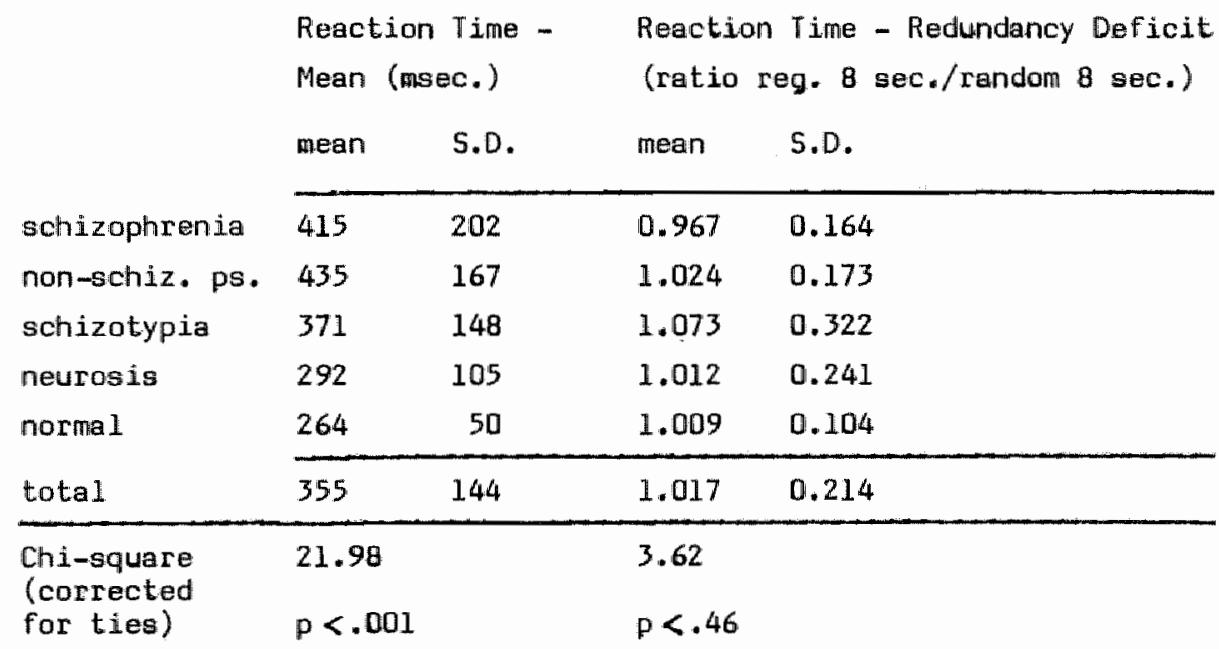


Table 21. Continuous Performance Test scores.

$\begin{array}{llll}\text { CPT Simple CPT Complex } & \text { CPT Simple CPT Complex } \\ \% \text { Dmisisions } \% \text { Omissions } & \% \text { Commissions } \% \text { Commissions }\end{array}$ mean S.D. mean S.D. mean S.D. mean S.D.

\begin{tabular}{|c|c|c|c|c|c|c|}
\hline schizophrenia & 12.7010 .83 & 18.7016 .06 & 3.40 & 3.53 & 3.25 & 6.29 \\
\hline non-schiz. ps. & $15.70 \quad 15.11$ & $22.70 \quad 17.77$ & 4.65 & 5.08 & 6.10 & 12.17 \\
\hline schizotypia & $12.60 \quad 15.05$ & $16.30 \quad 17.97$ & 4.30 & 7.34 & 2.70 & 7.31 \\
\hline neurosis & 6.7510 .23 & 10.9014 .68 & 2.25 & 1.68 & 3.40 & 5.74 \\
\hline normal & $1.00 \quad 1.17$ & $3.10 \quad 3.21$ & 2.00 & 1.72 & 0.65 & 0.81 \\
\hline total & 9.7511 .65 & 14.3414 .98 & 3.32 & 4.43 & 3.22 & 7.41 \\
\hline $\begin{array}{l}\text { Chi-square } \\
\text { (corrected } \\
\text { for ties) }\end{array}$ & $\begin{array}{l}29.39 \\
p<.001\end{array}$ & $\begin{array}{l}28.27 \\
p<.001\end{array}$ & $\begin{array}{l}3.25 \\
p<.5\end{array}$ & & $\begin{array}{l}16.20 \\
p<.00\end{array}$ & \\
\hline
\end{tabular}

normals and each of the patient groups, and between schizotypics and non-schizophrenic psychotics. The Mean Reaction Times are significantly different between normals or neurotics on the one hand, schizotypics or psychotics on the other hand, but not between normals and neurotics, or between schizotypics and psychotics. Roughly the same pattern is found for the omission scores of both CPT versions, although here the differences between normals and neurotics are statistically significant. Among the electrophysiological variables the mast significant difference is found for CNV Cz Early Component, between normals and schizophrenics. Differences between schizophrenics and the other patient groups are also significant, although to a lesser degree. For CNV $\mathrm{Cz}$ Late Component differences are less significant and only sa between schizophrenics and normals or neurotics. The other electrophysiological variables do not demonstrate a aimple pattern, although several significant differences exist between diagnostic groups. Reaction Time Redundancy Deficit and CPT Simple Version Commission score are outliers in this company, and SPEM Total seems a poor discriminator too. 


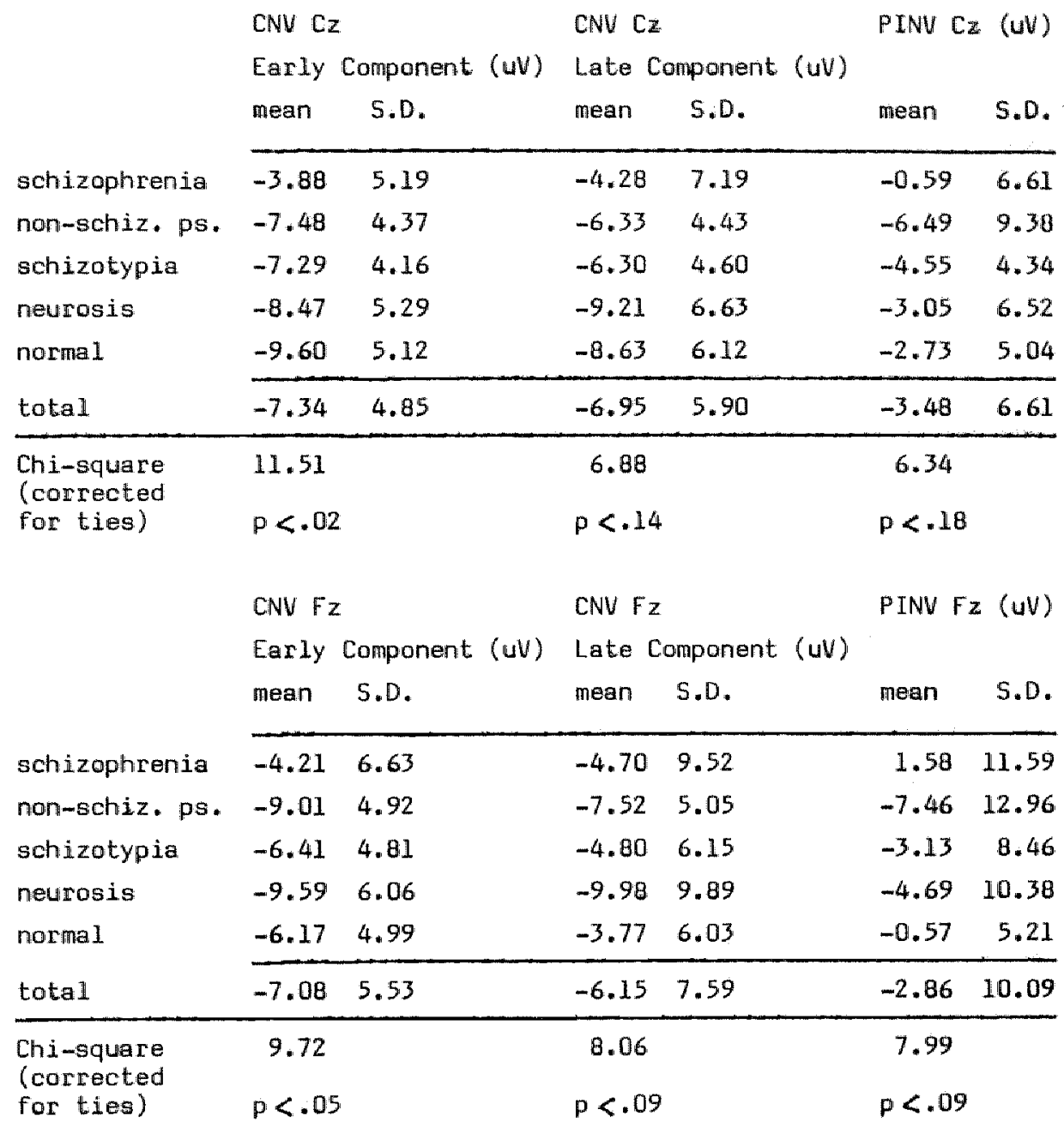


Table 23. Kruskal-Wallis one-way ANovA: Attentional variables.

Significance of differences between diagnostic groups. (Chi-square, corrected for ties).

- = non-significant $(p>.10) .(1=$ normal, $2=$ neurosis,

3 = schizotypal personality disorder, $4=$ non-schizophrenic psychosis, 5 = schizophrenia).

SPEM Total

$\begin{array}{llllllllll}1 & 1 & 1 & 1 & 2 & 2 & 2 & 3 & 3 & 4\end{array}$
vs. Vs. Vs. Ws. vs. vs. Ws. vs. Ws. vs. $\begin{array}{llllllllll}2 & 3 & 4 & 5 & 3 & 4 & 5 & 4 & 5 & 5\end{array}$

\section{SPEM Best Cycle:}

$\begin{array}{lllllllllll}.07 & - & .02 & - & - & - & - & - & - & - \\ .02 & .01 & .001 & .003 & .10 & - & .02 & - & - & - \\ - & .002 & .001 & .001 & .04 & .005 & .02 & - & - & -\end{array}$

RT Ried. Def.

CPT Simple Om. $.02 .001 .001 .001-.01 .03$

CPT Simple Com. CPT Compl. Om. $.02 .001 .001 .001-.005 .05 \quad .08$ CPT Compl. Com. .08 - .001.003 - $\quad$ - $\quad .02 .09$ CNW CZE.C. - $-\quad-.002-.02-.03 \quad .04$ CNW CZ L.C. CNW FZ E.C. - - - .05 - - .03 - - CNV FZL.C. PINU CZ PINU FZ

$\begin{array}{rrrrrrrrrr}.06 & - & .09 & - & - & - & .03 & .06 & - & .03 \\ .02 & - & .05 & - & .07 & - & - & - & - & - \\ -.0 & - & - & - & - & - & - & - & .04 & .04 \\ 10 & - & .02 & - & - & - & - & - & - & .06\end{array}$


Fiqure 5. Smooth Pursuit Eye Movements scores.

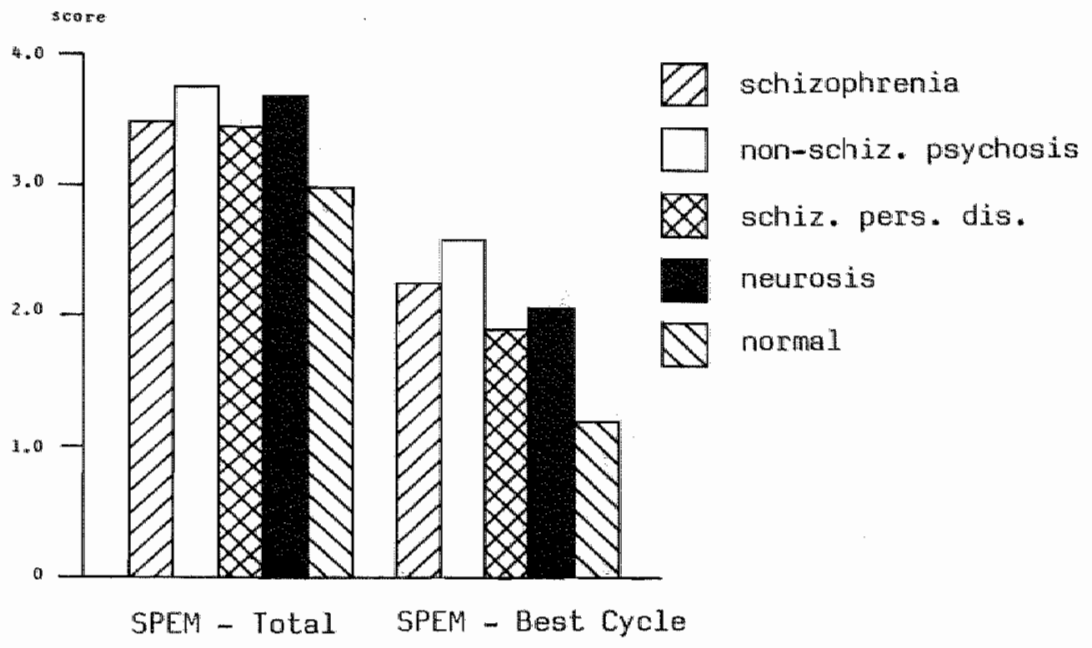

Fiqure 6. Reaction Time scores.

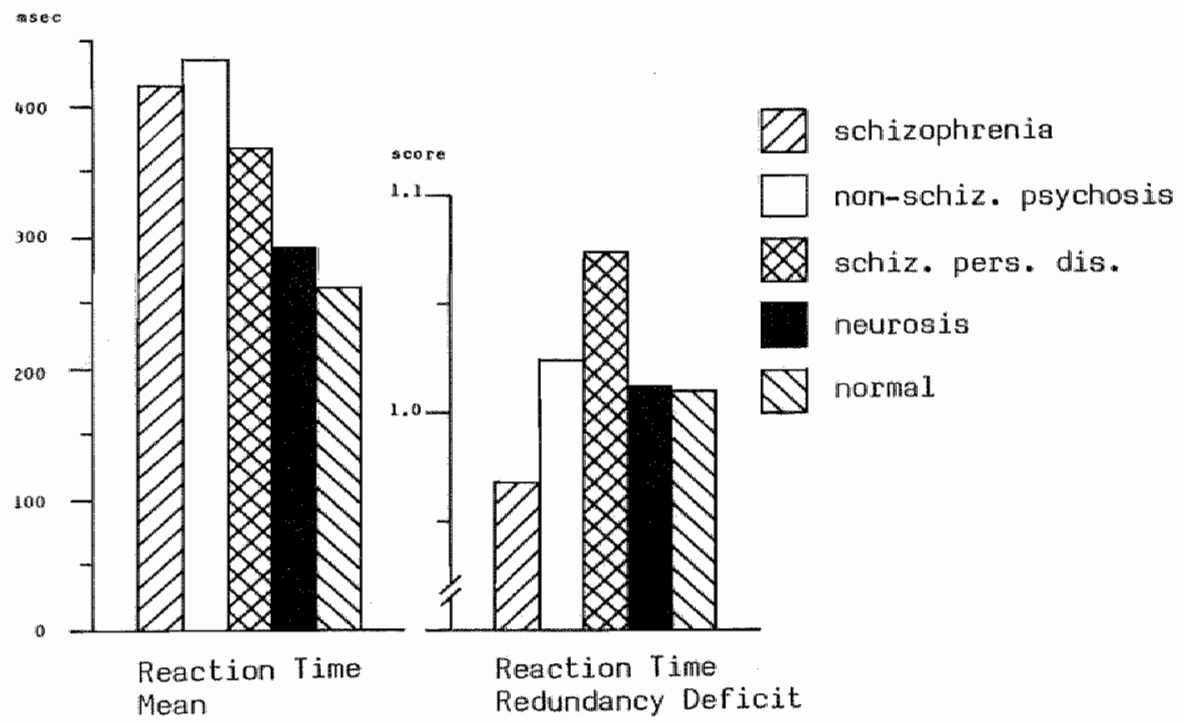




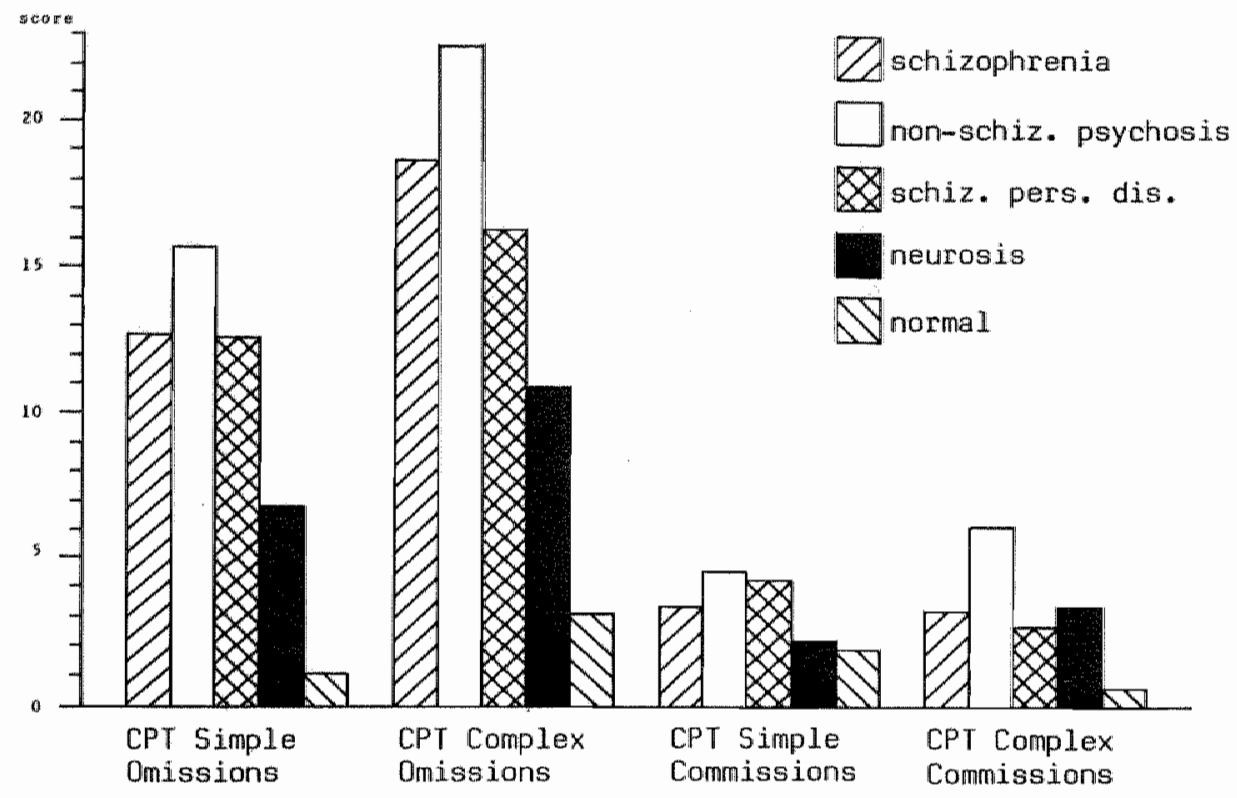

Figures 9-12 show some Smooth Pursuit Eye Movements patterns with varying degrees of disturbance. The original as well as the filtered records are presented. Figure 9 shows a nearly perfect pattern, the other figures show increasingly less good performances. Figure 13 presents the Mean Reaction Times, regular and irregular series, 2, 4, and $a$ seconds preparatory intervals, of the various diagnostic groups. Figures 14-17 show some Contingent. Negative Variation curves. Sl and S2 denote warning and imperative stimulus respectively. Figure 14 represente a 'normal' CNV. In Figure 15 the CNV amplitude is very low. Figures 16 and 17 show Post Imperative Negative Variations. These figures differ in their accentuation of early (Figure 16) or late (Figure 17) component of the CNV. Figure 18 presents the mean CNV's (and PINV's) with standard deviations, central and frontal derivations, of all diagnostic groups. 


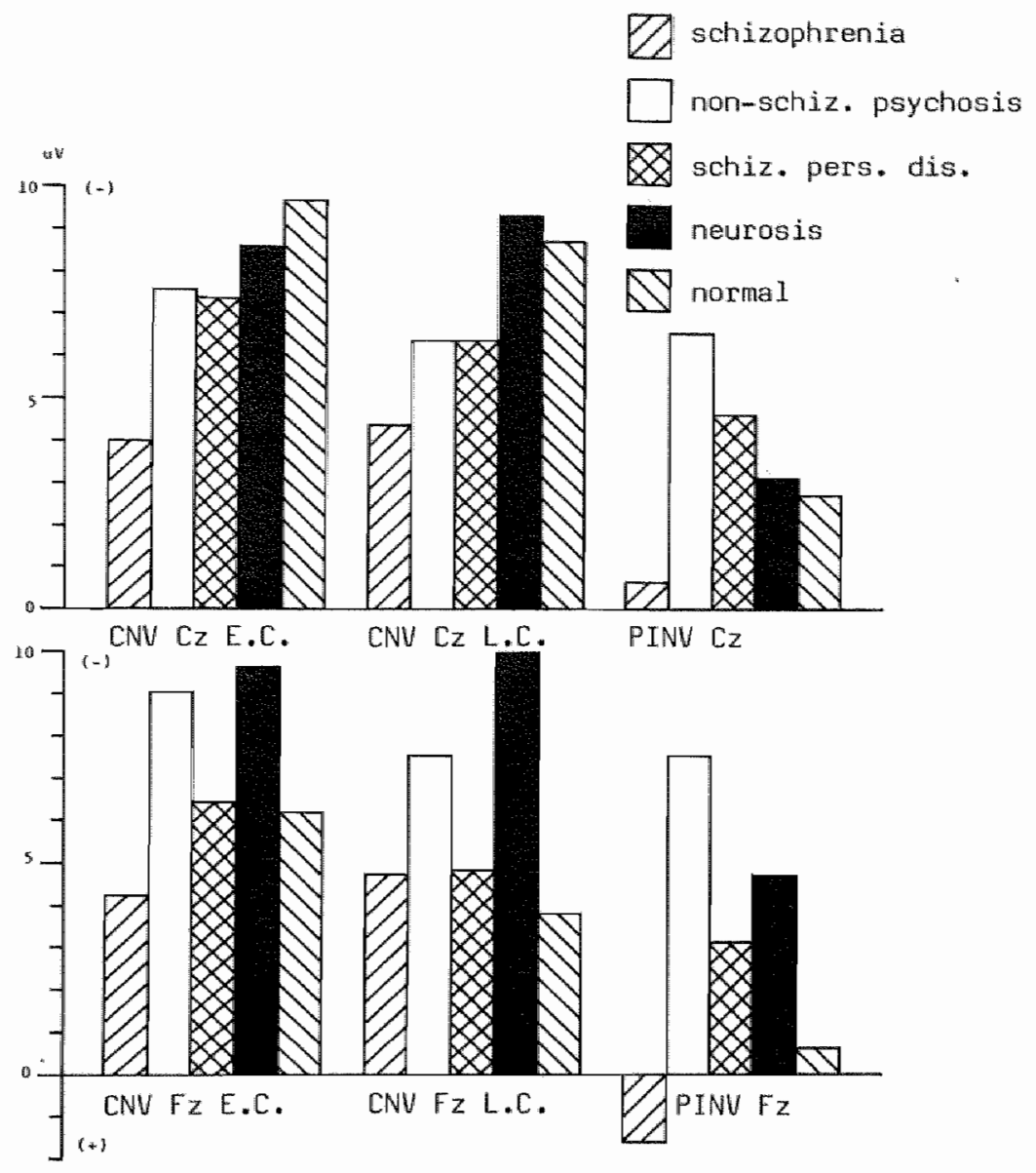


Figures 9-12. Smoath Pursuit Eye Movements patterns:

oriqinal and filtered records with varying degrees of disturbance.

\section{Figure 9.}

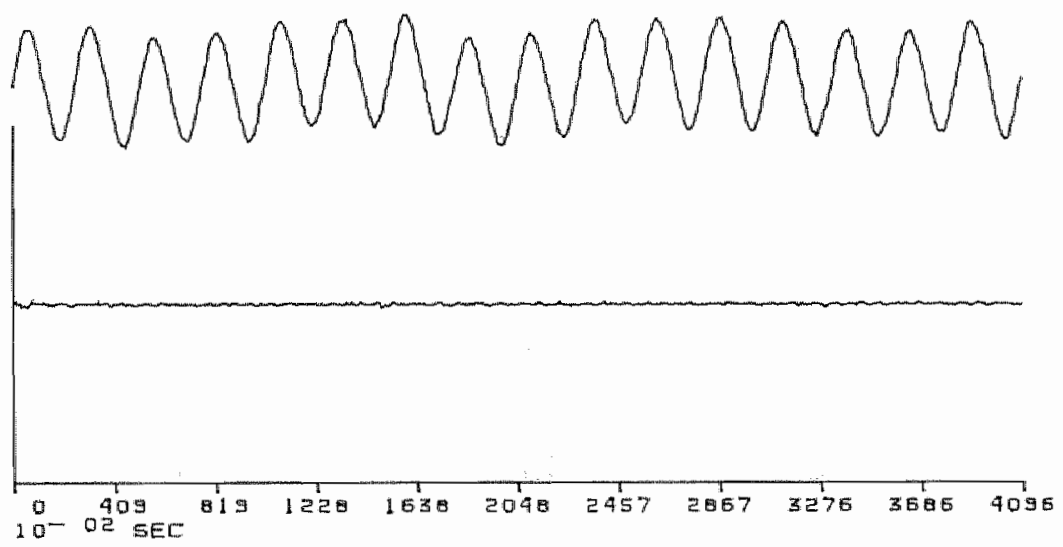

Fiqure 10.

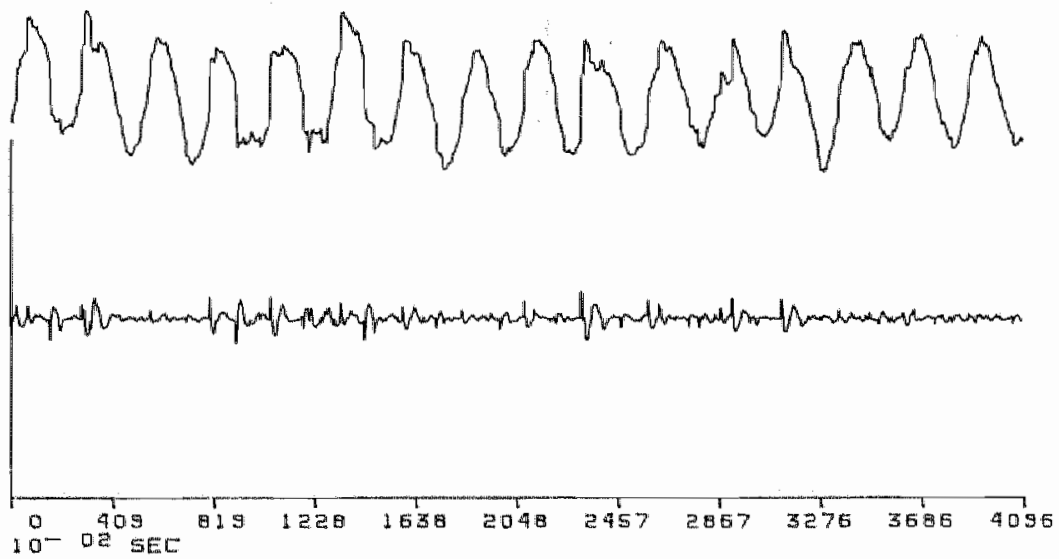


Figure 11.

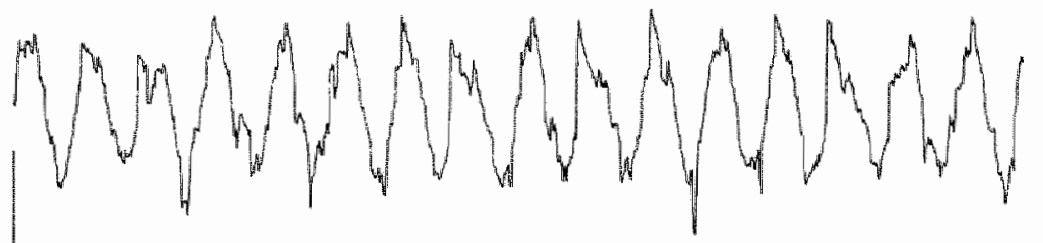

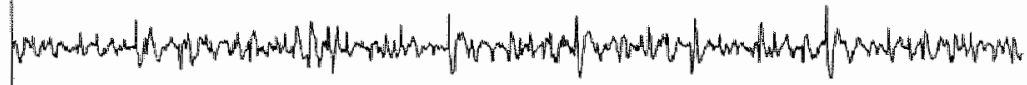

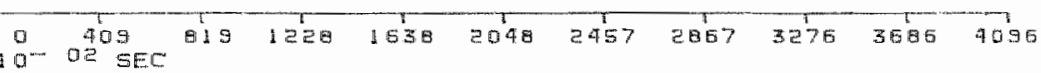

\section{Fiqure 12.}

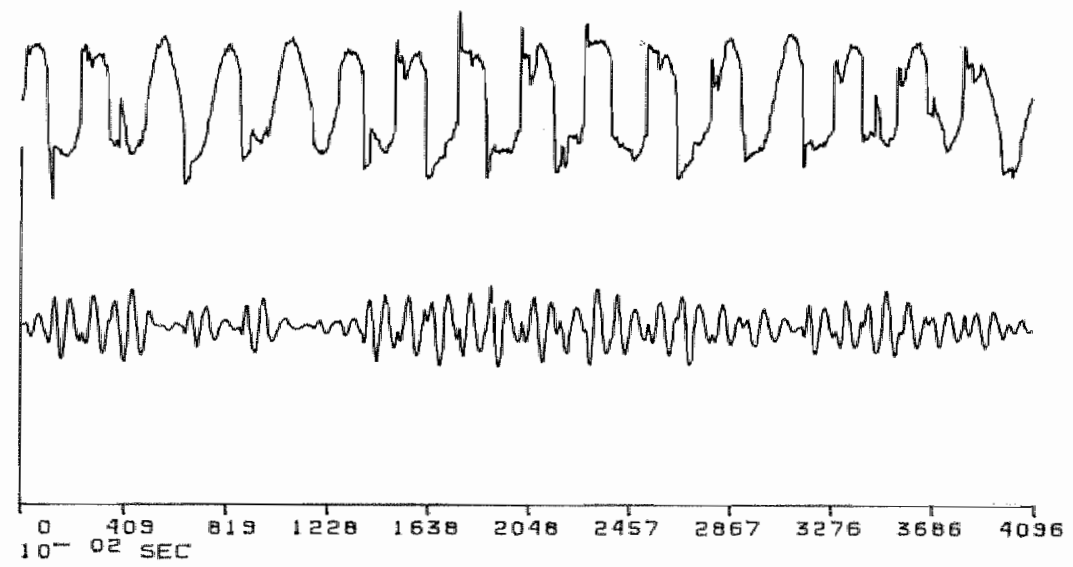


Elqure 13. Mean Reaction Times of the diagnastic groups:

\section{reqular and irrequiar series.}

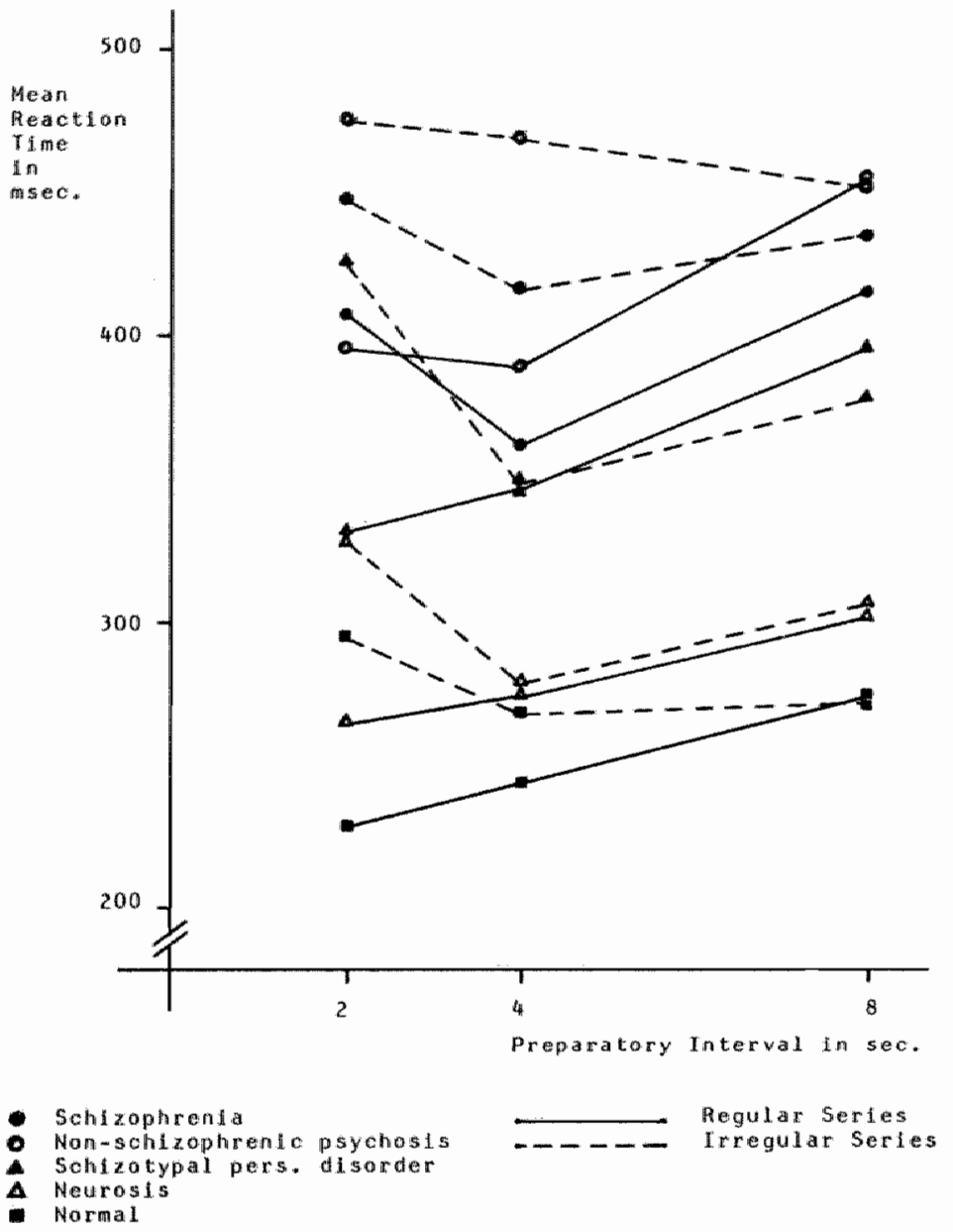


Eiqures 14-17. Contingent Negative Variation curves with varying degrees of disturbance.

Eiqure 14.

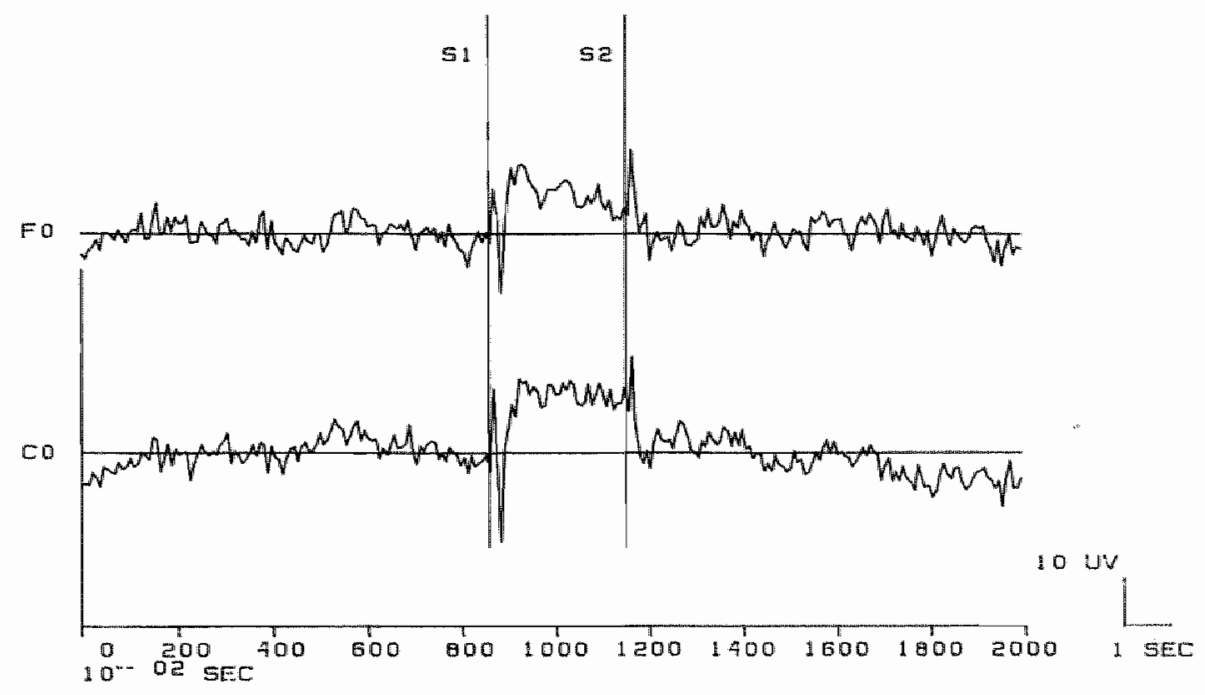

Fiquire 15.

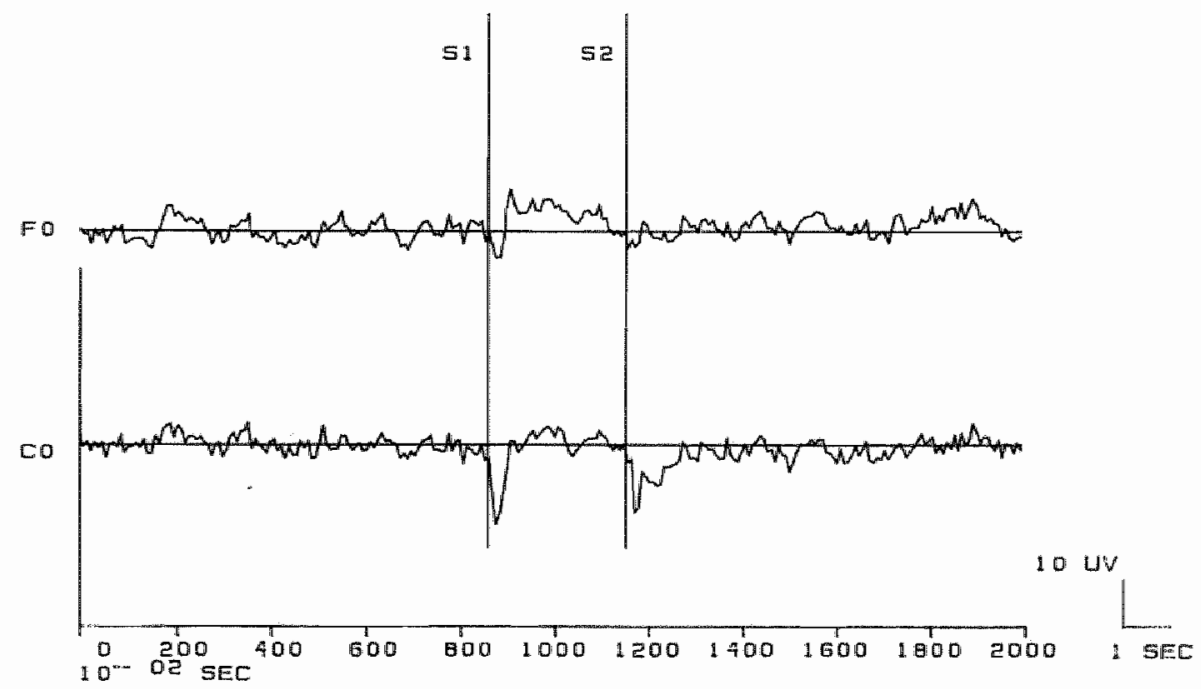


Fiqure 16.

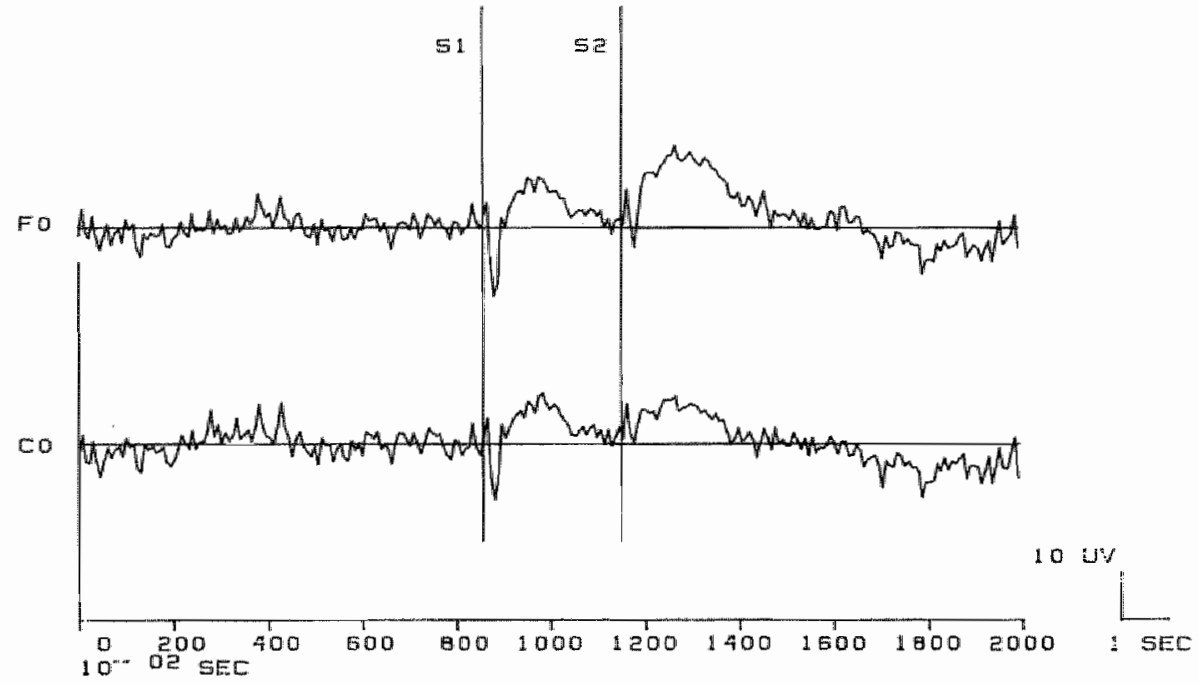

Figure 17.

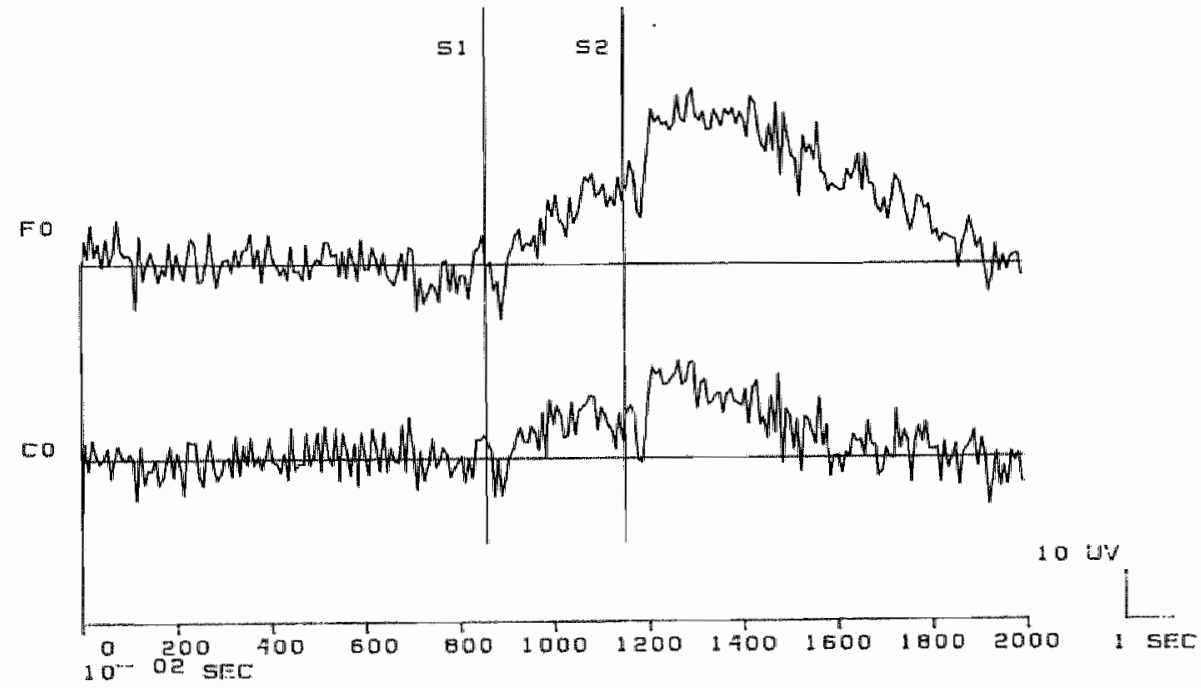


Fiqure 18. Mean CNV's (and PINV's) with standard deviations of the diagnostic groups, frontal and central derivations. $1=$ normal, 2 = neurosis, 3 = schizotypal personality disorder, 4 = non-schizophrenic psychosis, 5 = schizophrenia. Scale: 10 w $=$ I

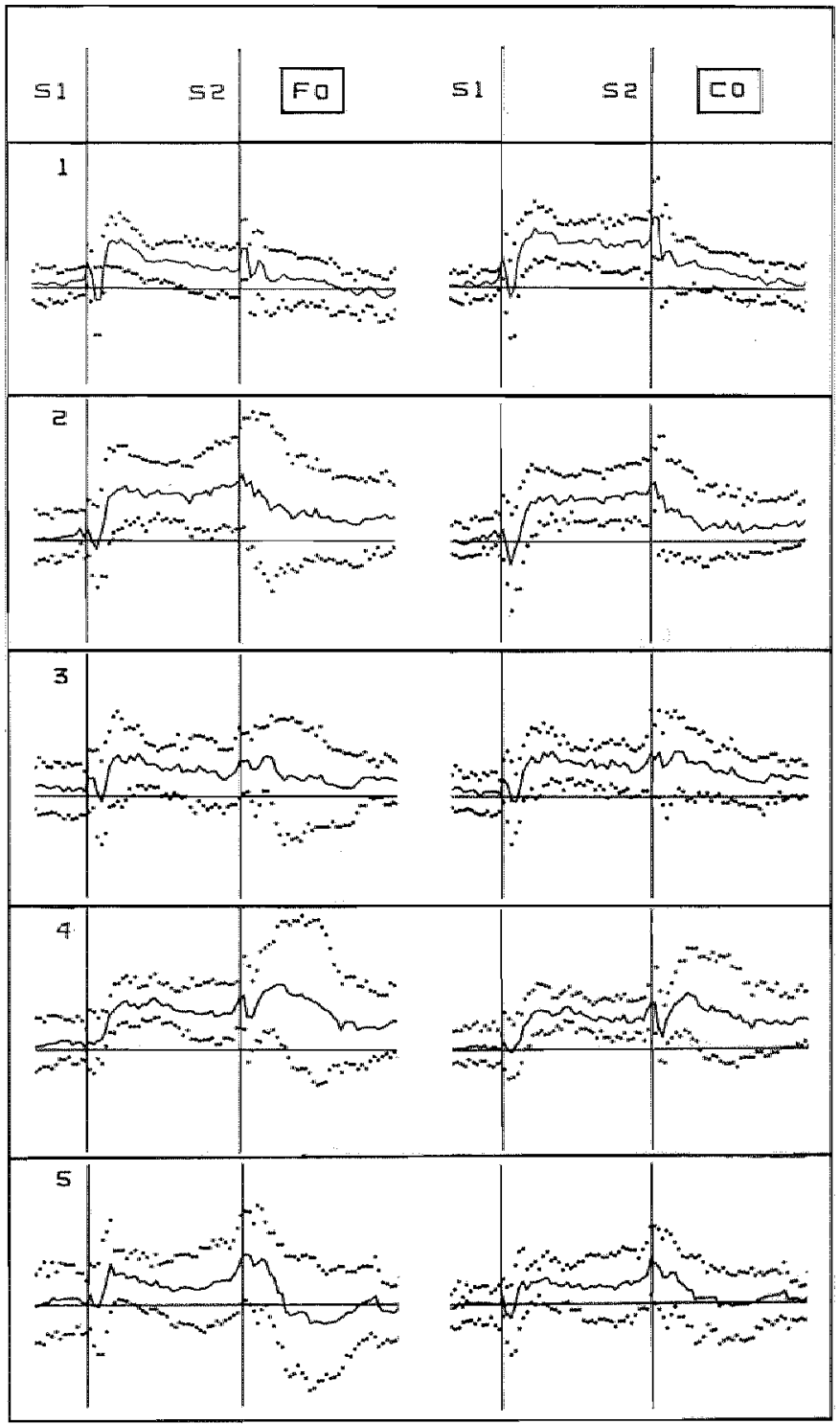


The results of stepuise multiple regression analysis of the attentional variables, with severity of diagnosis as the criterion variable, are presented in Table 24. This set of variables explains 34.8 percent of the variance. The first six variables are the most important ones, and the other variables appear to add little to the variance explained by this set. It is noteworthy that lov ranking

Table 24. Stepwige Multiple Regression Analysis.

\section{Attentional variables.}

criterion variable: rank ordered psychiatric diagnoses.

\begin{tabular}{cllll}
\hline Step No. Attentional varibble & $R$ & $R^{2}$ & $\begin{array}{l}\text { Standardized partial } \\
\text { Regression Coeff. }\end{array}$ \\
\hline 1 & Mean Reaction Time & .404 & .163 & .111 \\
2 & CNV CZ Early Component & .458 & .210 & .374 \\
3 & CPT Simple Omissions & .499 & .249 & .219 \\
4 & CNV Fz Early Component & .531 & .282 & -.138 \\
5 & SPEM Best Cycle & .539 & .291 & .285 \\
6 & SPEM Total & .563 & .317 & -.251 \\
7 & PINV Cz & .569 & .323 & -.329 \\
8 & PINV Fz & .574 & .329 & .245 \\
9 & Reaction Time Red. Def. & .578 & .334 & -.094 \\
10 & CPT Simple Commissions & .582 & .338 & .058 \\
11 & CNV Fz Late Component & .584 & .341 & -.242 \\
12 & CNV Cz Late Component & .588 & .345 & .227 \\
13 & CPT Complex Omigsiono & .589 & .347 & -.087 \\
14 & CPT Complex Commissions & .590 & .348 & .048
\end{tabular}

electrophysiological variables do nevertheless show relatively high partial regression coefficients. Relatively low partial regression coefficients are given to Mean Reaction Time, Reaction Time Redundancy Deficit (negative sign!) and, generally, the CPT scores. It is interesting to note the sign reversals between both SPEM measures 
(SPEM Total showing a negative sign) and between the central and frontal CNV and PINV measures (CNV Fz measures and PINV $\mathrm{CZ}$ showing negative signs). These results contrast with the results of the univariate analyses, from which SPEM Total, CNV Fz measures and PINV's did not emerge as good indicators of severity of diagnosis.

\section{Discriminant analyses.}

The same questions (a)-(d) posed earlier (section 7.1 .2 ) regarding the diagnostic variables are considered of interest here.

In the discriminant function of psychotics versus other patient groups (Table 25) Mean Reaction Time is clearly the most important variable. A different picture is shown by the discriminant function of psychotics versus (neurotics + normals). Four electrophysialogical variables stand in the first rank here (both PINV measures and both CNV Late Components). Electrophysiological variables have also relatively large weights in the other discriminant functions (Tables 26 and 27) although Mean Reaction Time is again the first variable selected by the stepwise procedure. The difference in sign between $\mathrm{Cz}$ and $\mathrm{Fz}$ derivations, CNV as well as PINV, is noteworthy. This tendency is present in all discriminant functions. Central CNV derivations always point in the direction of the clinically more disturbed diagnostic category, while frontal derivations point in the opposite direction. There is also a consistent pattern in the signs of the PINV's. Whenever one or both PINV measures are included in a discriminant function, the $\mathrm{Cz}$ derivation has a negative sign, and the $\mathrm{Fz}$ derivation has a positive sign. This finding replicates the results of the multiple regression analysis. Generally the highest ranking performance variables are both SPEM scores and Mean Reaction Time. There is also a consistent difference in sign between SPEM Total and SPEM Best Cycle. SPEM Best Cycle points in the direction of the more disturbed category, while SPEM Total chooses the opposite direction. Mean Reaction Time ranks high in the discriminant functions. Reaction Time Redundancy Deficit does not score high, and CPT scores have relatively modest positions in the discriminant functions. 
Iable 25. Stepeise digcriminant analyeis:

attentional variables.

Groups: (newrotics + schizotypics) versus (non-schizophrenic psychatics + achizophrenics).

\begin{tabular}{|c|c|c|c|}
\hline $\begin{array}{l}\text { Step } \\
\text { number }\end{array}$ & $\begin{array}{l}\text { Variable } \\
\text { entered }\end{array}$ & Rao's V & $\begin{array}{l}\text { Change in } \\
\text { Rao's } v\end{array}$ \\
\hline
\end{tabular}

$\begin{array}{lllll}1 & \text { Mean RT } & 6.79 & 6.79 & .009 \\ 2 & \text { CNV CZ E.C. } & 8.57 & 1.77 & .18\end{array}$

Standardized discriminant function coefficients:

$\begin{array}{ll}\text { Mean Reaction Time } & .75 \\ \text { CNV CZ Early Component } & .46\end{array}$

Correctly classified: $60 \%$ (Negative sign points to first category)

When single diagnostic groups are compared, using only the set of performance variables, no essentially new patterns emerge. Reaction Time Redundancy Deficit appears in the foreground in schizophrenics versus non-schizophrenic psychotics. It is the only variable selected here, but the correctly classified percentage is negligible applying this discriminant function, being only 52.5 percent. Comparing single diagnostic groups using only the set of electrophysiological variables shows generally the same pattern as the discriminant functions described arlier. Sign reversal between $C Z$ and Fz PINW's and CNV components, and between CNV and PINU measures, is apparent here too. The only deviation from the pattern described previously is found in the discriminant function of schizophrenics versus non-schizophrenic psychotics: CNU Fz Early Component demonstrates a positive sign here. Both components of the CNU are represented, but, like in the discriminant functions including the complete set of attentional variables, it is uncommon to find early as well as late components of the same CNV derivation in one single discriminant function. 
Presumably, when one of both components is already included, the other component of the same derivation does not add much to the discriminating power of its predecessor.

Table 26. Stepwise discriminant analysis:

attentional variables.

Groups: (normals + neurotics + schizotypics) versus (non-schizophrenic psychotics + schizophrenics).

\begin{tabular}{lllll}
$\begin{array}{l}\text { Step } \\
\text { number }\end{array}$ & $\begin{array}{l}\text { Variable } \\
\text { entered }\end{array}$ & Rao's V & $\begin{array}{l}\text { Change in } \\
\text { Rao's } V\end{array}$ & $\begin{array}{l}\text { Sign, of } \\
\text { change }\end{array}$ \\
\hline 1 & Mean RT & 15.02 & 15.02 & .000 \\
2 & SPEM Best C. 18.84 & 3.82 & .05 \\
3 & SPEM Total & 22.73 & 3.89 & .05 \\
4 & CNV CZ E.C. & 25.99 & 3.26 & .07 \\
5 & CNV Fz E.C. & 28.91 & 2.92 & .09 \\
6 & PINU CZ & 30.63 & 1.72 & .19 \\
\hline
\end{tabular}

Standardized discriminant function coefficients:

CNV $\mathrm{Cz}$ Early Component

SPEM Best Cycle

SPEM Total

$-.52$

Mean Reaction Time

.41

CNV Fz Early Component

$-.40$

PINV $\mathrm{CZ}$

$-.25$

Correctly classified: $69 \%$ (Negative sign points to first category)

The questions can be answered as follows:

(a) The best discriminating attentional variable between patients showing schizophrenia-like conditions and other (neurotic) patients and normals is CNV Cz Early Component, but Mean Reaction 
Time lis the first variable gelected by the stepuise procedure (Table 27 ).

(b) The best discriminating attentional variable between psychotic patients and non-psychotic patients plus normals is again CNV CZ Early Component, but again Mean Reaction Time, and in addition both SPEM measures, are selected earlier (Table 26).

(c) The best discriminating attentional variable between psychotic and non-psychotic patients is Mean Reaction Time (Table 25 ).

(d) The bost discriminating attentional variables between more and less disturbed patients or normals are generally the electrophysiological measures. The sign reversals between central $(\mathrm{Cz})$ and frontal $\left(\mathbb{F}_{Z}\right)$ measures, and between CNV and PINW are consistently found when psychiatrically more and less disturbed groups are compared.

\section{Influence of sex and age.}

Sex and age appear to exert some influence on the attentional variables, but only on the performance variables. SPEM Best Cycle scores are higher in women than in men $(p<.05)$, and correlate also positively with age $(p<.05)$ in the total sample. Mean Reaction Time is in the total sample significantly higher in women than in men, and is also significantly correlated with age (both $p<.001$ ). Reaction Time Redundancy Deficit is higher in men than in women $(p<.05)$ in the total sample). Finally, CPT Comission scores are significantly correlated with age in the total sample $(p<.05)$.

SPEM and Reaction Time are psychomotor attentional tasks. The influence of age and sex on psychomotor performance is well known. Age has been found to be the most important single factor influencing psychomotor performance. After a gradual development in the early years of life speed is maintained in the middle years until a gradual decline in great age. Sex differences are cansidered to reflect the disparity of muscular strenght and speed (discussion in King, 1975). Concerning eye tracking, the evidence on age and sex influences as 
Table 27. Stepwise discriminant analysis:

attentional variables.

Groups: (normals + neurotics) versus (schizotypics + non-schizophrenic psychotics + schizophrenics).

\begin{tabular}{lllll}
$\begin{array}{l}\text { Step } \\
\text { number }\end{array}$ & $\begin{array}{l}\text { Variable } \\
\text { entered }\end{array}$ & Rao's V & $\begin{array}{l}\text { Change in } \\
\text { Rao's V }\end{array}$ & $\begin{array}{l}\text { Sign. of } \\
\text { change }\end{array}$ \\
\hline 1 & Mean RT & 19.35 & 19.35 & .000 \\
2 & CPT Simple Om. & 25.56 & 6.21 & .01 \\
3 & CNV Cz E.C. & 29.08 & 3.52 & .06 \\
4 & PINV Cz & 33.60 & 4.52 & .03 \\
\hline
\end{tabular}

Standardized discriminant function coefficients:

CNV Cz Early Component $\quad .53$

Mean Reaction Time $\quad .44$

CPT Simple Omissions $\quad .40$

PINV $\mathrm{Cz} \quad-.39$

Correctly classified: $71 \%$ (Negative sign points to first category)

reflected in the current literature seems inconclusive at the moment (see Chapter 5.2.1).

However, when the level of statistical significance is set at .10, the differences between diagnostic gropus (Kruskal-Wallis one-way ANDVA) are still significant in both younger and older subjects (total sample split across the median, categories $20-32$ and 33-59 years of age), and in men as well as in women, for the variables SPEM Best Cycle and Mean Reaction Time. The difference between diagnostic groups for Reaction Time Redundancy Deficit, which is insignificant in the total sample, reaches statistical significance $(p<10)$ when determined in men only. The difference between groups remains insignificant for CPT Simple 
version Commission scores when analyzed in younger and older subjects separately, and becomes insignificant for CPT Complex version Commission scores when determined in the older category.

\section{Conclusion.}

The best indicators of severity of psychiatric diagnosis among the performance variables are SPEM Blest Cycle, Mean Reaction Time, CPT Simple Version Omission score, and CPT Complex Version Omission score. Among the ellectrophysiological variables only the $\mathrm{CNV} \mathrm{Cz}$ measures appear associated with severity of psychiatric diagnosis. Hypothesis IV is thus partly confirmed. According to the univariate analyses Reaction Time Redundancy Deficit has clearly to be considered an outlier, while SPEM Total plays a minor role, and the electrophysiological CNV Fz and PINV measures do not show clearly interpretable associations with rank ordered psychiatric diagnoses.

The associationel pattern of SPEM Best Cycle, Mean Reaction Time, and CPT Omission scores with psychiatric diagnosis suggests that the sample of non-schizophrenic psychotic patients in this investigation is generally somewhat more disturbed than the schizophrenic sample. This results was not predicted, because schizophrenia by chronicity being included in its definition was supposed to represent the generally more disturbed patients. There may have been slight sampling biases, because chronically hospitalized patients were not included in the investigation, and perhaps because schizophrenic patients, with poor social skills, are more essily hospitalized or referred to treatment facilities than equally disturbed mon-schizophrenic patients with a higher level of social competence. CNV Cz Early Component and to a somewhat lesser degree CNV $\mathrm{Cz}$ Late Component are the electrophysiological variables that appear significantly associated with severity of diagnosis. Only the electrophysiological measures show some significant differences between both psychotic groups. But more interesting than the confirmation that the attentional measures are associated with serious psychiatric disturbance - schizophrenic and non-schizophrenic psychosis - is the association with degree of 
psychopathology in general, whether psychotic or not. Generally schizotypics appear to occupy an intermediate position between psychotics and neurotics, and neurotics perform poorer than normals.

Reaction Time Redundancy Deficit disqualifies itself in this investigation as a useful indicator of psychosis or, generally, of degree of psychiatric disturbance. Its only prominent place is in the discriminant function of schizophrenics versus non-schizophrenic psychotics, but this function discriminates hardly between these diagnostic groups (and its sign does not point to the schizophrenic category). It might be that the method chosen in this study is not sufficiently comparable with the embedded set procedure as used by, for instance, Steffy and coworkers (see Chapter 5.2.2). On the other hand the method chasen here is largely identical to the procedure which originally demonstrated the crossover phenomenon, and both procedures - Shakou's regular and irregular series and Steffy's embedded sets - are generally considered interchangeably.

The criterion advacated by Steffy (1980) for the definition of Redundancy Deficit - a positive difference score of the raction time of the regular long PI duration trials minus the reaction time of the irregular long $P I$ duration trials of more than 25 msec. - , when applied to the data in this investigation, provides the following results. In the schizophrenic group only 4 subjects fulfil this criterion, and in the neurotic and normal groups 5 subjects. The numbers of subjects qualifying for Redundancy Deficit according to this criterion are highest in the schizotypic $(N=B)$ and non-schizophrenic psychotic group $(N=9)$. The resulting difference between groups is not statistically significant $\left(x^{2}=4.395,4 \mathrm{df}\right.$.). Thus, again the relation between schizophrenia and Reaction Time Redundancy Deficit is not confirmed, but there is a tendency for non-schizophrenic psychotics and schizotypics to show Redundancy Deficit as defined here. This definition is not satisfactory, however, as the possibility of confounding influence of differences in duration of reaction time is not excluded. The results cast heavy doubt upon the usefulness of Reaction Time Redundancy Deficit as an indicator of 
psychiatric, especially schizophrenic psychotic, disturbance. The findings referred to in Chapter 5.2.2, and partly summarized by Steffy (1980), auggesting a special relevance of Redundancy Deficit to the schizophrenia concept, are not confirmed by the results of the present invertigation, although one interesting correlation deserves to be mentioned: this otherwise outlier-variable is associated to some degree $(p<.10)$ with duration of hospitalization.

Mean Reaction Time has confirmed itself in this investigation as a useful indicator of poychiatric disturbance. In its association with psychlatric diagnosis this measure suggests more or less a separation between psychotics and schizotypics on the one hand, neurotics and normala on the other hand. Although the schizotypics occupy an intermediate position, they are closer to the psychotics.

SPEM Best Cycle shows itself a clear indicator of degree of psychilatric disturbance, but the results concerning SPEM Total are in contrast with the studies reviewed in Chapter 5.2.1, all of which used measures of eye tracking based on a series of cycles. In this investigation only the differences between non-schizophrenic psychotice and normels, and between neurotics and normals reach statiatical significance using this SPEM measure. It should be noted that the diacrimination between diagnostic categories found by other investigators was not always satisfactory (e.g., Shagass, Amadeo and Overton, 1974; Pivik, 1979 a; b; Levin, Lipton and Holzman, 1981 ). SPEM Best Cycle is a measure not previously analysed. It was included because of its suspected better relationahip with 'core' deficits than SPEM Total. This conjecture is confirmed by the results. The Best Cycle measure of eye tracking may be related in practice to the improved eye tracking obtained by monitoring periodic changes of the target (e.g., Shagass, Roemer and Amadeo (1976), and others, discussed in Chapter 5.2.1), but this asaumption should be tested directly, of course. The behavior of these two SPEM measures in the multiple regression and discriminant analyses is very interesting. While SPEM Best Cycle points consistently in the direction of the clinically more disturbed category, whether psychotic or not, SPEM Total points, also 
consistently, to the opposite category. This means, the less the difference between the scores of SPEM Best Cycle and SPEM Total, the more it indicates a high degree of psychiatric disturbance. Psychotics, it seems, are oloser to their limits of performance than less seriously disturbed psychiatric patients. They are really not able to perform much better than indicated by their SPEM Total score, while less disturbed patients or normals are better able to improve on their average eye tracking quality.

Concerning the Continuous Performance Test: from the multiple regression analysis only CPT Simple Version Omission score emerges as a clear indicator of degree of psychiatric disturbance. The Commission scores are apparently less reliable indicators of degree of disturbance. In the discriminant functions CPT measures do usually not play a leading role. When present they nearly always point to the more severely disturbed category. In their pattern of associations with peychiatric diagnosis CPT Omission scores and Mean Reaction Time correspond with each other. As already mentioned, both are alao positively intercorrelated in all diagnostic groups (except normals: speed of motor reaction does perhape not influence the CPT scores in this group to the same degree as it does in the patient groups).

Concerning the electrophysiological variables there is a striking difference between their someuhat confusing associational pattern with psychiatric diagnoses as demonstrated by the correlational analyses and analyses of variance, and their position in the multiple regression and discriminant analyses. CNV $C z$ measures appear correlated with severity of diagnosis, the Early Component to a higher degree than the Late Component. Early and Late Components seem to share much information, as it is uncommon to find both represented in one single discriminant function, when belonging to the same CNV derivation. However, the most otriking feature of the multivariate analyses, so far as electrophysiological variables are concerned, are the sign reversals between CNV and PINV measures and between $\mathrm{Cz}$ and $\mathrm{Fz}$ derivations. Both $\mathrm{Cz}$ and $\mathrm{Fz}$ measures are represented in the discriminant functions, but $\mathrm{CNV} C \mathrm{Cz}$ measures point to 
the clinically more disturbed category, while CNV $\mathrm{Fz}$ measures point to the opposite direction. Usually the $\mathrm{CZ}$ measures get the higher loading. In all discriminant analyses in which both psychotic groups are put together in one category, and are being compared with combinations of other diagnostic groups, the general pattern emerges, and this pattern is also shown by the multiple regression analysis. These resulta suggest that the clinically most disturbed patients, especially psychotico, are characterized by low central CNV levels and relatively high frontal. CNV levels, while the opposite characterizes less disturbed and normal subjects. PINU's do also show sign reversal. Whereas, Iike the Fz CNV derivations, the PINV's did not show significant associations with severity of psychiatric diagnosis in the univariate analyses, their importance in this respect is demonstrated by the multivariate analyses. Post imperative negativity of the central CNV's consistently points to the more disturbed diagnostic category, while frontal PINU's choose to opposite direction. This suggests that the clinically most disturbed patients, especially psychotic patients, are characterized by a high degree of post imperative negativity in their central CNV registration, while their post imperative negativity is relatively low in frontal CNW's.

The results are in accordance with those of other investigators, revilewed in Chapter 5.2.4, for CNV $\mathrm{Cz}$ and PINV $\mathrm{Cz}$ measures. The deviant behavior of $\mathrm{FZ}$ measures is an entirely new finding. The association between CNV $\mathrm{Cz}_{2}$ amplitude and degree of psychiatric disturbance rather than psychotic disturbance is in line with the reports of, among athers, Dongier (1973) and Abraham, McCalium and Gourlay (1976). The relation between PINV Cz and degree of psychiatric disturbence was less clear in earlier studies, although Dongier, Dubroviky and Garcia-Ril1 (1974) found the prevalence of PINV's in neurotic patients halfway between those in normals and psychotics.

Compared with the performance variables, the electrophysiological variables seem to be generally the somewhat better discriminators between diagnostic categories. Dverall there is no convincing evidence that schizophrenic psychotic patients differ in important ways from 
mon-schizophrenic psychotic patients regarding the attentional variables (although there are differences at the electrophyajological level). In other words, the DSM-III distinction between schizophrenic and non-schizophrenic psychosis does not seem to make much sense at the level of attentional functioning. This finding is in contrast with the results with respect to the diagnostic variables Affective Flattening, Social Isolation and Premorbid Asociality, but is in agreement with the association between Psychoticism and psychiatric diagnosis. Schizotypal personality disorder emerges as a diagnostic category that is closer related to both psychotic categories, concerning attentional functioning, than the neurotic category. This finding is in agreement with recently published results on backward masking in schizotypic, schizophrenic, and control groups (Braff, 1981; Saccuzzo and Schubert, 1981). Backward masking offers a measure of processing speed, and these studies have offered support to the slow information processing hypothesis for schizophrenia and, to a lesser degree, schizotypal personality disorder. Like these backward masking studies, the results of the present investigation provide support to the hypothesized relationship between schizatypal personality disorder and schizophrenia, but at the attentional level this relation seems not limited to schizophrenia as defined by DSM-III , but to extend to other functional non-affective psychoses. 
Multiple regression analysis: diagnostic and attentional variables.

Table 28 shows the results of stepuise multiple regression analysis, with severity of psychiatric diagnosis as the criterion variable, using both sets of diagnostic and attentional variables. The explained

Table 28. Stepwise Multiple Reqression Analysia.

Diagnostic and attentional variables.

criterion variable: rank ordered paychiatric diagnoses.

\begin{tabular}{cllll}
\hline Step No. Diagnostic variable & $R$ & $R^{2}$ & $\begin{array}{l}\text { Standardized partial } \\
\text { Regression Coeff. }\end{array}$ \\
\hline 1 & Affective Flattening & .473 & .223 & .211 \\
2 & SPEM Best Cycle & .563 & .317 & .284 \\
3 & Neuroticism & .599 & .359 & .210 \\
4 & Lie & .628 & .395 & .262 \\
5 & Psychoticism & .671 & .450 & .235 \\
6 & SPEM Total & .690 & .477 & -.269 \\
7 & CNV Cz Early Component & .705 & .497 & .250 \\
8 & CNV Fz Late Component & .715 & .511 & -.207 \\
9 & Social Isolation & .720 & .518 & .117 \\
10 & Extraversion & .724 & .525 & .131 \\
11 & CPT Simple Commissions & .729 & .531 & .077 \\
12 & CNV Fz Early Component & .731 & .534 & -.091 \\
13 & Mean Reaction Time & .732 & .536 & -.082 \\
14 & CPT Complex Omissions & .734 & .538 & .037 \\
15 & Reaction Time Red. Def. & .734 & .539 & -.049 \\
16 & PINV Cz & .735 & .540 & -.233 \\
17 & PINV Fz & .739 & .547 & .213 \\
18 & CNV Cz Late Component & .741 & .549 & .167 \\
19 & Premorbid Asociality & .741 & .550 & .042 \\
20 & CPT Complex Commissions & .742 & .550 & .030
\end{tabular}


variance, being 34.8 percent using the attentional set and 44.4 percent using the diagnostic set, appears to rise to 55.0 percent when both sets of independent variables are combined. Cenerally, the diagnostic variables, notably Affective Flattening, Psychoticism, Neuroticism and Lie, appear to be somewhat better indicators of severity of psychiatric diagnosis than the attentional variables in these analyses. Mean Reaction Time and CPT Simple Version Omission score, among the best predictors in the analysis including only the attentional set, are dropped or ranked very low in the analysis including diagnostic as well as attentional variables. Their predictive capacity is apparently accounted for by the high ranking diagnostic variables.

\section{Relations between diagnostic variables and attentional variables.}

In the total sample (Table 29) the correlations between SPEM scores and Affective Flattening, Social Isolation and Premorbid Asociality are significant $(p<.05)$ anly for some correlations that include the Best Cycle score. Mean Reaction Time correlates somewhat higher with these diagnostic measures, but Reaction Time Redundancy Deficit does not show any significant correlations. The omission scares of the Simple and Complex CPT Versions are all significantly correlated with the "achizophrenia variables". Among the electrophysiological variables mainly the central CWV measures correlate significantly with some of these diagnostic variables.

The correlations betureen Psychoticism and attentional variables do not reach high levels of significance in the total sample. Of both SPEM measures only SPEM Total correlates to a marginally significant degrea (p<.10) with Psychoticism score. Mean Reaction Time, both CPT omission scores and all CNV measures correlate significantly at the 5 percent level with Psychaticism. SPEM Best Cycle, Reaction Time Redundancy Deficit and both PINV measures do not correlate significantly with Psychoticiam. 
Table 29. Spearman Correlation Coefficients.

Iotal sample: Diagnostic variables versus Attentional variables.

$\begin{array}{llllll}\text { Aff. Soc. Prem. } & P & E & N & \text { L } \\ \text { Flatt. Isol. Asoc. } & & & \end{array}$

\begin{tabular}{|c|c|c|c|c|c|c|c|}
\hline SPEM Total & -.04 & .08 & .06 & .13 & .00 & .12 & $.18^{*}$ \\
\hline SPEM Best Cycle & .10 & $.20^{*}$ & $.19^{*}$ & .08 & -.07 & $.2 \mathbb{1}^{*}$ & $.24^{* *}$ \\
\hline RT Mean & $.35^{* * *}$ & $.26^{* * *}$ & $.22^{*}$ & $.17^{*}$ & $-.18^{*}$ & .15 & $.38^{* *}$ \\
\hline RT Red. Def. & -.05 & .02 & .06 & .02 & .08 & -.14 & -.04 \\
\hline CPT Simple Om. & $.38^{* * *}$ & $.31^{* * *}$ & $.26^{* *}$ & $.21^{*}$ & -.09 & $.31^{* * *}$ & .14 \\
\hline CPT Simple Com. & .15 & -.09 & .02 & .15 & .03 & .07 & .06 \\
\hline CPT Compl. Om. & $.37^{* * *}$ & $.29^{* * *}$ & $.26^{* *}$ & $.20^{*}$ &.- .15 & $.24^{* *}$ & $.22^{*}$ \\
\hline CPT Compl. Com. & $.19^{*}$ & $.18^{*}$ & .08 & .06 & .05 & .16 & .09 \\
\hline CNV CZ E.C. & $.24^{* * *}$ & .10 & $.26^{* *}$ & $.17^{*}$ & -.12 & .16 & .16 \\
\hline CNU $C z \mathbb{L} . \mathrm{C}$. & $.19^{*}$ & .09 & $.17^{*}$ & $.21^{*}$ & -.14 & $.20^{*}$ & $.23^{* * *}$ \\
\hline CNU Fz E.C. & .11 & .01 & .15 & $.19^{*}$ & -.09 & -.02 & .05 \\
\hline CNV FZ L.C. & .09 & .01 & .04 & $.17^{*}$ & -.12 & .06 & .07 \\
\hline PINU $C Z$ & .03 & -.11 & .00 & .08 & .08 & .02 & .12 \\
\hline PINU $F Z$ & -.01 & -.04 & -.03 & .12 & .01 & -.06 & .05 \\
\hline
\end{tabular}

There is a significant positive correlation in the total sample between SPEM Best Cycle and Neuraticism. Extraversion score does not seem to be related to SPEM quality. Mean Reaction Time is negatively correlated with Extraversion and marginally significantly $(p<.10)$ positively correlated with Neuroticism. There is also a trend to negative correlation between Reaction Time Redundancy Deficit and Neuroticism. Correlations of CPT Omission scores with Extraversion are low and hardly significant, but the correlations with Neuroticism are positive and significant. Between CPT Commission scores and scores on the EPQ scales correlations do not exceed chance level. The correlations between electrophysiological measures and Extraversion score are negligible. The correlations of both CNV CZ measures with 
Neuroticism are higher and significant, but the other electrophysiological variables show no association with Neuroticism.

Within-group analyses.

Within-group analyses were applied in order to correct for confounding influences of psychiatric diagnosis as a moderator variable. Overall, in the within-group analyses (Tables 29a-29e, in Appendix 9) the diagnostic measures Affective Flattening, Social Isolation and Premorbid Asociality do not correlate significantly with the attentional variables. This contrasts with the correlational pattern in the total sample. However, the neurotic group is an exception, as here a relatively high number of significant correlations is found, in the predicted direction, and not limited to one or two of the attentional variables, but involving the whole set. Concerning Psychoticism, within diagnostic groups significant correlations are very limited, only both psychotic groups showing significant positive correlations with SPEM Total. Within diagnostic groups correlations between Mean Reaction Time and Extraversion score are positive in the schizophrenic and schizotypic groups, and to a significant degree so in the latter category. These correlations are negative, hovever, in the other diagnostic groups, and all of these correlations reach significance levels. In the correlational pattern of Reaction Time Redundancy Deficit a parallellism between the schizophrenic and the schizotypic group is also notable: in both a significant positive correlation is found with Extraversion scare, and a significant negative correlation with Neuroticism score. In the schizophrenic group both of these correlations are even highly significant $(p<.01)$. This pattern is lacking in the other diagnostic groups.

\section{Conclusion.}

The total sample shows clear associations between the "schizophrenia variables' and the attentional variables, but within groups significant associations appear limited to the neurotic patients. Eysenck's concept of Psychoticism as a measure of the predisposition 
to develop a psychotic disorder receives some support from the analysis in the total sample of its associations with the attentional variable: , SPEM Best Cycle, Reaction Time Redundancy Deficit and both PINW measures do not participate in these associations. However, the within-group associatione between Psychoticism and the attentional varlables are very poor. Clearly, explanations concerning associations between 'schizophrenia variables' and attentional variables as weII as between Psychoticism and attentional variables should be tentative. Poychiatric diagnosis serves as a moderator variable, as both diagnostic and attentional - variables are correlated with severity of diagnosis, but the within-group analyses are handicapped by the small number of subjects. Among the correlational patterns of the other EPQ scales some interesting aspects have to be noted. The results suggest complex interactions between Extraversion and Neuroticism scores on the one hand, Reaction Time performance on the other hand, but exclusively within schizophrenic and achizotypic patient samples.

The association found between Neuroticism and amplitude of the central CNV agrees with the results abtained in other patient studies (McCallum and Walter, 1968; Fukui, Nakamura, Kadobayashi and Katoh, 1978). Cenerally, the associations in the total sample between Neuroticism and attentional variables are comparable with those between Psychoticism and attentional variables. Differences are found with respect to SPEM measures and CNV Fz measures. Concerning the Psychoticism scale, the problem of consistency of the Dutch translation of the EPQ has been mentioned. It is not possible to establish the extent to which this problem influences the results. Nevertheless, as already discussed, Eysenck"s (1976) fürst method of walidation of the Psychoticism scale, comparing Peychoticism scores in psychotic patients with scores in other selections of patients and in normale, results in this investigation in support to Psychoticism as relited to psychosis, but only within a paychiatric patient sample. His second method of validation, correlating Psychaticiam scores with scores on 'objective and laboratory tests', does not support the superiority of Psychoticism to Neuroticism with respect to assaciation with attentional dyafunctioning in the total sample. The within-group 
analyses do hardly support Eysenck's assumptions.

\section{Cluster analyses.}

Exploratively, cluster analyses were applied to the variables in this investigation. Clustering methods are advocated by numerical taxonomiste for the purpose of classification. This objective appraach to classification may become more important in psychiatry because of the widely recognized unsatisfactory position of current diagnostic practice. The attentional variables which take part in the present investigation are likely candidates for such new approaches to classification.

Cluster analysis is a statistical procedure which creates a classification from a set of data. The procedure became mare popular after the introduction of numerical taxanomy by Sokal and Sneath (1963). But the great number of different clustering methods that have been developed creates problems (Everitt, 1974). Blashfield (1976) has compared four important clustering methods in terms of their accuracy in salling data sets, generated by a mixture model. The highest accuracy values were obtained by the minimum variance method, also known as Ward's method (Ward, 1963). This is one of the most frequently used techniques (Blashfield and Aldenderfer, 1978). It is a hierarchical agglomerative method, which proceeds by a series of successive fusions of the entities into groups. Ultimately the method reduces the data to a single cluster containing all the entities. Ward's method is designed to generate clusters in such a way that the variance within the clusters is minimal. This clustering method was: used in this investigation in order to compare groups of subjects thus generated, with minimal intersubject variance, with diagnostic categorization. Hierarchical clustering methods imply no clear indicators to decide the number of clusters present in the data. But inspection of the clustering coefficients, representing a metric related to the amount of variance accounted for at each step of the hierarchical clustering process, may sometimes show 'jumps' in values which are out of proportion to previous changes. When the agglamerative clustering procedure forces two clustere together with rather unlike profiles the solution immediately preceding should be considered.

Cluster analysis, Ward's type, based on all attentional variables in this investigation, shows two plausible solutions. A seven-cluster solution (coefficient-change from 6.88 to 10.04) and a two-cluster solution (caefficient-change from 18.55 to 31.07 ). The results of both 
solutions are presented in Tables 30 and 31 . In the seven-cluster solution cluster 5 and cluster 7 have a total of only three subjects, and are probably to be ignored, representing 'outliers'. The cluster profiles of the two-cluster solution are shoun in Figure 19. The mean scores of both clusters on the diagnostic and attentional variables are plotted here in relation to the mean scores and standard deviations on these variables of the total sample.

Table 30. Cluster Analysis - All Attentional variables. Minimum Variance Method (Ward's), seven-cluster solution.

diagnostic numbers of subjects in the clusters group cl. 1 cl. 2 cl. 3 cl. 4 cl. 5 cl. 6 cl. 7

\begin{tabular}{lrrrrrrr}
\hline schizophrenia & 3 & 7 & 4 & 3 & 0 & 3 & 0 \\
non-3chiz, psychosis & 1 & 1 & 6 & 1 & 1 & 9 & 1 \\
schizotypia & 7 & 1 & 4 & 1 & 0 & 6 & 1 \\
neurosis & 6 & 2 & 3 & 4 & 0 & 5 & 0 \\
normal & 10 & 3 & 0 & 4 & 0 & 3 & 0 \\
\hline total & 27 & 14 & 17 & 13 & 1 & 26 & 2
\end{tabular}

Table 31. Cluster Analysis - All Attentional variables. Minimum Variance Method (Ward's), two-cluster solution.

$\begin{array}{lll}\text { diagnostic } & \text { number of } & \text { number of } \\ \text { subjects in } & \text { subjects in } \\ \text { group } & \text { cluster } 1 & \text { cluster } 2\end{array}$

schizophrenia 9

11

non-schiz. psychosis

11

9

schizotypia

14

6

neurosis

15

normal.

17 
Figure 19. Cluster profiles.

Cluster analysis including all attentional variables. Two-cluster solution.
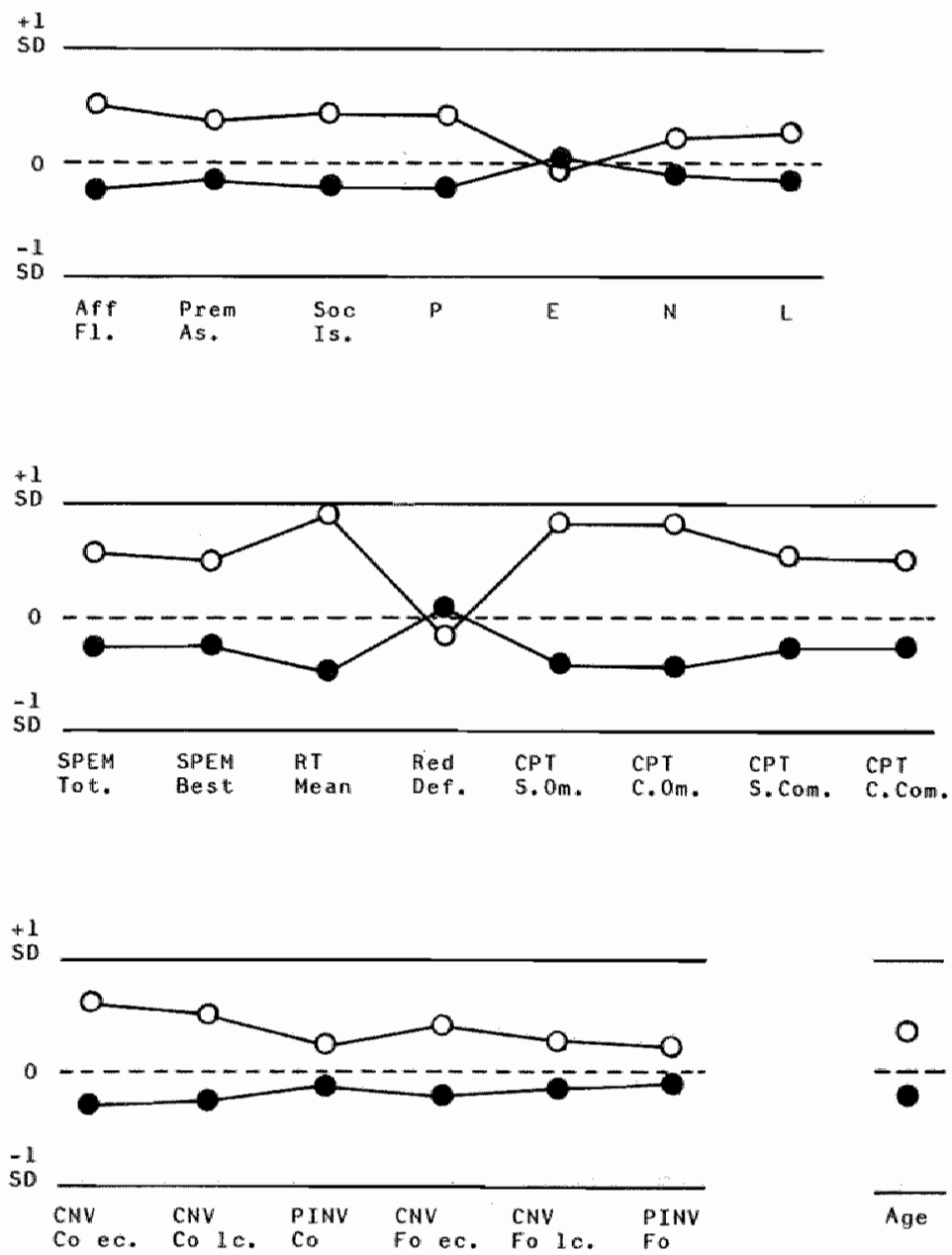
When cluster analysis is performed using the sets of electrophyolological and pexormance variables separately, different patterns are shown. The results of cluster analysis based on the performance variables show a tendency to separate the clinically more disturbed patients from normals and less disturbed patients, whereas the results of cluster analysis based on the electrophysiological wariables do not. It is noteworthy that in both analyses the change of coefficient is highest in the final step. (in both a threefold increase), auggesting that the level of two clusters should be preferred. However, when cluster analysis is performed using all electrophysiological as we1l as performance - variables, the final coefficient change is less impressive. The main dimensions of these gets seem to be confounded in cluster analysis based on both sets together.

The distribution of the 5 diagnostic groups in the two-cluster solutions shows a proportional representation of the psychotic patiente in both clusters in those analyses including the electrophysiological variables, alone or in combination with the performance variables. Among the other diagnostic groups the share in the "deviant' cluster Increases parallel to an increase in severity of paychiatric diagnosis, but only in those cluster analyses including the performance variables, alone of in combinatian with the electrophysiological variables. The minimum variance method including this set of variables does not create a sharp demarcation between psychotic and non-psychotic subjects, but does show a tendency to a separation of the total sample in clinically more disturbed and less disturbed clusters. This is in accordance with the tendency, found in other statistical analyses in this investigation, of the performance attentional variables to correlate with degree of psychiatric disturbance rather than with particular diagnoses (psychotic disorders). The relevance of the electrophysiological variables in this respect is not evident from the cluster solutions including only this set of variables. 
Table 32. Cluster Analysis - A11 Diagnastic and Attentional Variables. Minimum Variance Method (Ward's), tua-cluster solution.

\begin{tabular}{lll} 
diagnostic & number of & number of \\
group & subjects in & subjects in \\
\hline
\end{tabular}

schizophrenia

non-schiz. psychosis

schizatypia

neurasis

normal

$\begin{array}{rr}3 & 17 \\ 2 & 18 \\ 0 & 20 \\ 8 & 12 \\ 19 & 1\end{array}$

18

20

12

1

total

32

68

Cluster analysis including all variables - diagnostic as well as attentional - provides the most interesting two-cluster solution (Table 32). The profiles of these clusters are shown in Figure 20. Coefficients change gradually in this cluster analysis, the largest change being the final step from two clusters to one cluster (from 15.24 to 25.20 ). The addition of the diagnostic variables to the attentional variables does markedly improve the accordance with rank ordered psychiatric diagnoses, as could be expected. Psychotic and schizotypic patients are largely placed in one cluster, normala are largely in the other cluster, while neurotic patients take an intermediate position.

Traditional psychiatric classifications attempt to fulfill too many functions. This is primarily the result of the lack of a unifying concept of disease, in combination with the borderline position of psychiatry, halfway between medecine and psychology. The empirical approach needs more appreciation in psychiatry. Useful theories and real progress require precise measurements and definable operations. The results of the cluster analyses presented here support the assumption that in psychiatry a polythetic classification, based on the principles of numerical taxanomy (see Chapter 2.1), is likely to 
Fiqure 20. Cluster profiles.

Cluster analys is including all diagnastic and attentional variables. Tua-cluster solution.

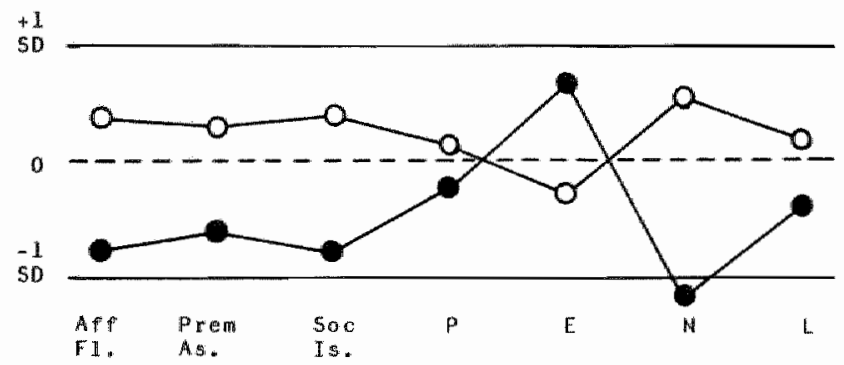

$+1$

$\mathrm{SO}$

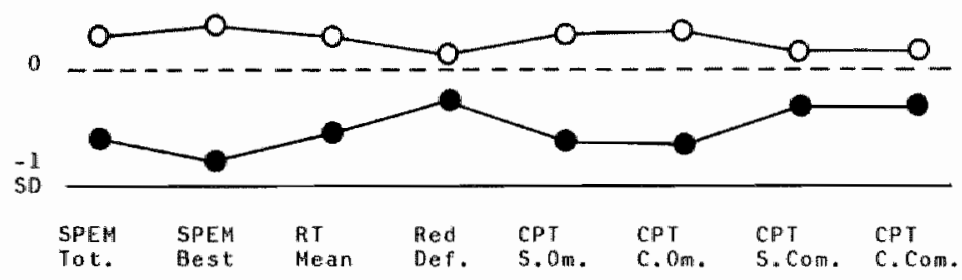

ot. II

$5 \mathrm{D}$
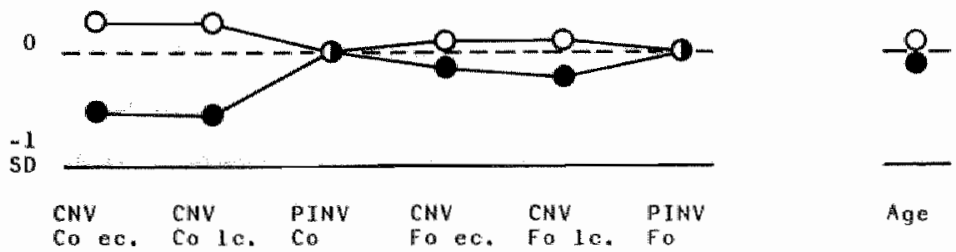

Co e. Co lo. Co

Fo ec. Fo le, Fo 
depart fram traditional conceptualizations in this field, and more so the less traditionally defining features are included in the selected characters. That the vast and complex field of clustering methods, and the lack of a logical system of clustering algorithms, remain weak points of numerical taxonomy should not be forgotten.

\section{General pattern.}

Generally the results of this investigation support the conception of psychiatric disturbance up to florid psychosis as a continuum. There are no sharp demarcations bietween the diagnostic categories distinguished in this investigation at the level of attentional performance. None of the attentional variables draws atrict dividing lines and differences are generally gradual across diagnastic groups. This continuum conception is illustrated by the results of Ward"s type clusstoring procedure. 
The heterogeneous and perhaps somewhat confusing impressions offered by the analyses of the interrelations between all those attentional and diagnostic variables should not obscure the central issue of the investigation presented here: the relevance of some objective indicators of attentional dysfunctioning to severity of psychiatric diagnosis, in particular to the clinical concept of schizophrenia and related disorders. The diagnostic variables are of secondary interest compared with the attentional variables in the frame of this study, but both sets represent alternatives to traditional categorical classifücations.

Smooth Pursuit Eye Movements.

The utility of SPEM measures as indicators of psychiatric disturbance has been confirmed, but SPEM Best Cycle appears a better discriminatar between clinically good and poor functioning patients than SPEM Total. It is significantly correlated with severity of psychiatric diagnosis, whille SPEM Total is not. The signs of the loadings an these two measures of Eye Tracking in the multiple regression and discriminant analyses indicate that the difference between both scorea has also significance to the discrimination between levels of psychiatric disturbance. The smaller the difference score, the more it indicates a high degree of psychiatric disturbance. In other words, clinically more disturbed patients are apparently less able to improve on their 
average eye tracking quality than lesa disturbed patients or normals.

The results of this investigation with respect to the discriminating power of SPEM measures can be combined in a simple way in a composite index, providing a single measure of eye tracking performance. Such an index should include SPEM Best Cycle score and the difference between rotal and Begt Cycle scores, as both are associated with severity of psychiatric diagnosis. The difference score can be range-corrected by

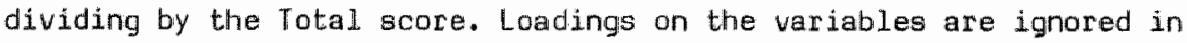
this aimple index. The SPEM index then becomes:

SPEM Index $=\frac{(\text { SPEM Total }) \cdot(\text { SPEM Best Cycle })}{(\text { SPEM Total })-(\text { SPEM Best Cycle })}$

This index maximizes individual differences on eye tracking performance and seems interesting to further studies. To illustrate the power of the index, the index scores obtained by applying it to the SPEM scores of the members of the diagnostic groups are shown in Table 33. The rank order of SPEM Best Cycle scores is preserved, except both psychotic groups which change places (the difference between these groups is to be ignored, however), but the distribution is widened, especially between psychotics and normals. The standard deviations are also increased relative to the mean scores, and show the same rank order.

Table 33. SPEM Index scores applied to the subjects in the patient and norma 1 qroups.

\begin{tabular}{lrrrrrr} 
diagnosis & \multicolumn{2}{c}{ SPEM Total } & \multicolumn{2}{c}{ SPEM Best C. } & \multicolumn{2}{c}{ Index score } \\
& Mean & S.0. & Mean & S.D. & Mean S.D. \\
\hline schizophrenia & 3.49 & 0.99 & 2.27 & 1.25 & 12.43 & 17.59 \\
non-schiz. psych. & 3.78 & 1.30 & 2.59 & 1.03 & 11.47 & 9.38 \\
schizotypia & 3.45 & 1.09 & 1.91 & 0.93 & 5.57 & 5.20 \\
neurosis & 3.70 & 1.34 & 2.06 & 1.22 & 7.45 & 8.12 \\
normal & 2.99 & 1.06 & 1.21 & 0.72 & 2.75 & 2.73
\end{tabular}


Reaction Time Performance.

Mean Simple Reaction Time is a good indicator of degree of psychiatric disturbance. This vell-known finding has been confirmed in this investigation. Reaction Time Redundancy Deficit, however, has appeared an outsider in the company of the attentional variables. It does not correlate with degree of psychiatric disturbance as formalized in psychiatric diagnosis, and it does not appear in the factor analysis of the attentional variables. When the definition of Redundancy Deficit as proposed by Steffy (1980) is applied, the non-schizophrenio psychotic group and the schizotypic group contain a higher number of subjects qualifying for Redundancy Deficit than the other groups, but the difference is not statistically significant, and in addition this criterion deserves criticism for confounding Redundancy Deficit with Reaction Time duration. Although the relations with diagnostic variables are not impressive either, there are some interesting trends in the analyses, which are not easy to interpret however. Within the samples of schizophrenic and schizotypic patients Reaction Time Redundancy Deficit is associated with high Extraversion score and with low Neuroticism score. But only within these 'schizaphrenic spectrum" categories. Within the total sample - patients plus non-patients Reaction Time Redundancy Deficit appears correlated with sex, being higher in men than in women, and with age, being higher in older subjects. Finally, high scores on this variable appeer to some degree associated with long duration of hospitalization. In other words, the typical high Redundancy Deficit scoring subject is an older man, with a history of many months or even years of psychiatric hospitalization, and when falling within the schizophrenic or schizotypic category, presenting himself as extravert and not subject to neurotic stress. Oniy the history of hospitalization reminds af the description of the typical Redundancy Deficit subject in earlier studies (e.g., steffy, 1980), in which especially process schizophrenics showed the deficit. In addition the correlational analyses within the set of attentional variables reveal an interesting trend. Within the neurotic sample, and to a lesser degree within the schizotypic sample, Reaction Time Redundancy Deficit is associated with other performance variables, 
notably SPEM measures and CPT Omission scores. Further study of these complicated patterns seems justified.

\section{Continuous Performance Test.}

The Omisaion sciores of both - Simple and Complex - CPT versions are associated with degree of psychiatric disturbance, but deficits are characteristic of psychotic and other serjous disorders in general rather than of schizophrenia in particular. This association is less clear in the case of the Commission scores, which are apparently less valid indicators of severity of psychiatric disturbance. Factor analysis of the set of attentional variables suggests that CPT Omission as well as Commission scores have much in common with simple (Mean) Reaction Time performance.

Contingent Neqative Variation and Post Imperative Negative Variation.

Anong the electrophysiological variables, the best indicator of degree of psychiatric disturbance, as defined by psychiatric diagnosis, seems CNV Cz Early Component, although CNV Cz Late Component is only slightly less good in this respect. Only the CNV Cz measures show clear assaciations with some performance variables. The analysis of the relations with the 'schizophrenia" and EPQ variables does not provide much additional information, although the associations of CNV measures with Psychaticism as well as Neuroticiam in the tatal sample is noteworthy. More interesting are the multivariate analyses. Whereas CNV $F_{2}$ measures and PINV measures did not seem useful indicators of psychiatrio pathology in univariate analyses, multiple regression and discriminant analyses show that the pattern is more complicated. Characteristic of the more disturbed patients appears to be a tendency of low central CNV amplitude in combination with relatively high frontal CNN amplitude. Multivariate analyses also reveal that PINV's are related to severity of psychiatric diagnasis, and again there is sign reversal between central and frontal derivations: the more disturbed patients show a tendency to high central and relatively low frontal PINV. There thus seems to be a tendency of frontal 
overactivity and central underactivity of the cerebrel cortex in the more disturbed psychiatric patients during the S1-52 interval of the CNV paradigm, in combination with more persistent activation in the central regions than in the frontal area. The frontal lobes are concerned with higher forms of planned behavior, of regulation of activation processes (Luria, 1980). Psychotics and other more disturbed patients do apparently need more activation of these structures in order to meet the demands of the test situation. This may be related to the supposediy stronger appeal to conceptually driven processing made by schizophrenics (and probably other rather disturbed patients) as referred to in Chapter 2.4. As the character of the PINW is still ambiguous (see Chapter 5.2.4), explanation of its distributional characteristice is risky. Perhaps it is justified to ascribe its higher central manifestation in more disturbed patients to motor activation processes, which apparently require more persistent ("spasmodic'?) cortical activation.

To combine in a simple way (ignoring loadings on the variables) the discriminating capacities of the CNV and PIN distributions in one single measure, an index may be constructed. This index should include central and frontal CNV and PINU measures. Although differences between Early and Late Components can probably be ignored, the Early Components may be preferable because of the slightly higher correlation of $\mathrm{CNV} \mathrm{CZ}$ Early Component with severity of diagnosis. Nevertheless the mean amplitude of Early and Late components may also be used. The following CNV index is thus constructed:

CNV Index = (CNV CZ Early Component - CNV FZ Early Component $)$ + (PINV $F_{Z}-$ PINV $C z$ ).

This index maximizes in a simple way individual differences on topographica 1 variations of CNV and PINV. Applying it to the CNV scores of the subjects in the diagnostic groups gives the results presented in Table 34. This table gives also the 'CNV and PINV difference components' of the index. 
Table 34. CWU Index scores app 1ied to the subjects in the patient and normial qroups

\begin{tabular}{lrrrrrr} 
diagnosis & \multicolumn{2}{l}{ CNW E.C. } & \multicolumn{2}{l}{ PINV } & \multicolumn{2}{l}{ Index score } \\
& \multicolumn{2}{c}{ Difference score Difference score } & \\
& Mean & S.D. & Mean & S.D. & Mean & S.D. \\
\hline schizophrenia & 0.34 & 3.26 & 2.16 & 6.16 & 2.50 & 6.29 \\
non-schiz. psych. & 1.53 & 3.74 & -0.97 & 6.14 & 0.56 & 5.30 \\
schizotypia & -0.88 & 3.31 & 1.42 & 6.50 & 0.54 & 6.36 \\
neurosis & 1.12 & 4.02 & -1.64 & 6.86 & -0.53 & 5.77 \\
normal & -3.43 & 2.92 & 2.16 & 3.08 & -1.27 & 2.92
\end{tabular}

The sign reversal between schizotypics and neurotics on the CNV Index is noteworthy, as is the exceptional position of the schizophrenic group, in particular when this group is compared with the nonschizophrenic psychotic group. It is interesting that the CNV + PINV distribution index shous the same rank order of diagnostic groups as the early components of the central CNV, while this is not the case with its 'components', the CNV and PINW 'differences': (CNU CZ Early Component - CNV Fz Early Component) and (PINV Fz - PINV $C z$ ). Unfortunately, differences between groups are not statistically significant on the CNV Index (Kruskal-Wallis One-Way ANOVA, P>.10). Its power as an indicator of psychopathology has to be established in further studies.

\section{Diagnostic variables.}

Affective Flattening and Psychoticism are high loading variables in the multiple regression and discriminant analyses, being indicative o: the more disturbed categories as determined by psychiatric diagnosis. The success of Affective Flattening is flattered by its inclusion in the criteria for schizophrenia and schizotypia (DSM-III), although its presence is not essential for these diagnoses. The distribution of Psychoticism and Neuroticism scores across diagnostic groups and their pattern of weights and signs in the discriminant functions suggests 
that Neuroticism is the better discriminator betueen patient and normal samples, whereas Psychoticism discriminates within a psychiatric patient sample between psychotic and non-psychotic patients. The multivariate analyses show that Extraversion, Soclal Isolation (like Affective Flattening also included in the polythetic sets of criteria for the DSM-III diagnoses of schizophrenia and schizotypia!) and Premorbid Asociality are apparently of relatively low importance as indicators of severity of psychiatric disturbance. On the other hand, the "social dysfunctioning" dimension seems particularly relevant to the schizophrenia construct, and it is probably this dimension in which differences between psychotics labeled schizophrenic and psychotics placed outside this category (by DSM-III) are most marked. To the degree that attentiona 1 dysfunctioning is considered a central characteristic of the "desintegration" dimension schizophrenic and non-schizophrenic psychotic patients, as defined by DSM-III, are not strikingly different from each other on this dimension in the present investigation.

\section{Resume.}

The results of this investigation support the utility of measures of attentional functioning as indicators of psychiatric disturbance, especially of psychosis. As far as measures of attention and information processing deficits are concerned, schizophrenia, defined according to DSM-III, does not emerge as a diagnostic category clearly distinguishable from other psychotic disorders. There are differences. between the schizophrenic and non-schizophrenic psychotic sample日 differences in electrophysiological measures and in mean scores on Affective Flattening and Premorbid Asaciality, for instance, but at the level of attentional performance these differences are poorly defined and generally negligible. In this investigation there is a trend suggesting that the non-schizophrenic psychotics are, on the whole, slightly mare disturbed than the schizophrenics, but the difference is very small indeed. This implies that the opposite position - schizophrenic psychatic patients being more disturbed than 
non-gchizophrenic paychotic patients - is not confirmed. That positio seemed feasible initially, because the definition of schizophremia inherent in DSM-III includes some degree of chronicity, whereas the criteria of the other psychotic category do not. Traditionally, of course, 'schizophrenia' is considered the most serious psychiatric diagnosis. As stated before, there may have been silight sampling biases.

The reaults suggest a two-component model of schizophrenia as it is traditionally conceptualized. The first component is shared with other peychotic conditions, and to a lesser degree with non-psychotic psychiatric conditions. It is labeled desintegration-proneness or vulnerability, and poor attentional performance ranks high among its characteristics. The second component is shared with other non-psychotic psychiatric canditions, but manifests itself most clearly when combined with high vulnerability. It consists of poor social competence skills. Whereas winerability is strongly genetically determined, poor social akills are largely due to defective learning experiences. The traditional 'poor prognosis' schizophrenia concept is characterized by high "loadings" on both components. Interactions between these components, representing essentially continuously distributed characteristics, can be assumed to lead to mutual reinforcement, and to create vicious circles and eventually paychotic breakdowns.

The results support the notion of psychiatric disturbance as a continuum of gradually increasing desintegration of mental functioning. Dyafunctions of attentional processes and information processing, as operationalized by the performance and electrophysiological methods in this investigation, represent global indications of disturbed mental functioning across the whole range, from relatively mild (neurotic) disturbance to psychosis. The poorer the attentional performance, the higher the probability the subject belonge to a diagnastic categary clinically recognized as paychotic. The patients clasaified as schizotypic fall in between the neurotic and psychotic patient samples. The attentional performance of this 
category does not suggest a special relationship with the schizophrenic category, but some diagnostic measures (Affective Flattening, Social Isolation, Premorbid Asociality) do. The Ward's type hierarchical agglomerative clustering procedure illustrates the continum conception of psychiatric disturbance. It shows, not unexpectedly, that the addition of clinical and psychometrito (diagnostic) measures to the attentional measures improves markedly the accordance with rank ordered psychiatric diagnases.

Psychoticism as defined by the $P$ scale of Eysenck's Personality Questionnaire is no alternative to 'traditional' diagnostic description of clinically recognizable psychiatric disturbance. It may be a useful addition, but although it correlates positively with severity of psychiatric diagnosis, it does not discriminate between patient and normal samples in the present investigation, and there is hardly any evidence that it is associated with attentional dysfunctioning within diagnostic categories. Firm conclusions as to the validity of the $P$ scale are not allowed, hovever, as the consistency of the Dutch version of the $P$ scale has yet to be established.

Among the attentional variables the best discriminators between less and mare disturbed psychiatric patients are, according to the resulto of this investigation: SPEM Best Cycle, Mean Simple Reaction Time, Omission scores of Simple and Complex CPT Versions, and CNV Cz Earily Component. The explanatory capacity of two of these variables is significantly enlarged when the scores on these variables are considered in combination with the scores on related variables. SPEM Best Cycle should be cansidered in combinetion with SPEM Total, and CNV CZ measures should be considered in combination with CNV Fz measures, Likewise, PINN $\mathrm{Cz}$ and PINV Fz measures have to be considered together in order to fully appreciate their relevance. Reaction Time Redundancy Deficit is the only real outlier among the attentional variables in that apparently this measure does not share with the other attentional variables an association with level of psychiatric disturbance as formalized, in psychiatric diagnosis. But there are 
indicationg of more complicated relationships within as well as across diagnostic categories with other, diagnostic and attentional, variables. Future reaearch should study these complex associational patterns in more detail.

Another area deserving attention in future studies is the analysis of the distribution of slow potential shifts, in particular the CNV, acrosis the scalp. This analysis seems to promise new chances to electrophysiological correlatea of attention in the search for parameters of psychiatric disturbance. In the meantime it is remarkable that researchers applying other techniques (traditional EEG, cerebral blood flow measurement, and positron enission tomography) have found diminished frontal activity in schizophrenics, as compared with other patients or normals, while the results presented here point to the opposite direction. Perhaps chronicity is a relevant variable in this respect, the patients atudied here not being very chronic. In addition the specific task requirements af the CNV paradigm will be important. Studies in a resting state (e.g. cerebral blood flow, by Franzen and Ingvar, 1975; cerebral glucography with pasitron tomography, by Buchsbaum, Ingvar, Kessler, et al., 1982) are not comparable with the present study for that reason. Also worth further study is the extent to which measures of optimum attentional performance can be obtained in psychiatric patients, particularly in psychotic patients. The analysis of SPEM performance in the diagnostic groups in this investigation suggests that this approach may be more fruitful than the mere registration of attentional performance vithout distinction between average and optimum performance. Although the heterogeneous nature of the arousal concept is likely to hamper such research, parallel registration of attentional performance and level of arousal may further increase the understanding of 'real' deficits. A clear distinction at the attentional level between schizophrenic and non-schizophrenic psychotics (DSM-III) seems implausible, but there are indications of attentiona 1 differences between both categories, which deserve more study. These indications are offered by the between-group differences on CNV as well as CNV-index scores, and by the interactions between Reaction Time measures (Mean and Redundancy 
Deficit) and Extraversion and Neuroticism scores in the schizophrenic and schizotypic groups.

\section{Some remarks on therapeutic aspects.}

It is still insufficiently recognized that as a rule schizophrenic and other psychotic patients are handicapped by deficiencies in attention and information processing. This study illustrates again that many (schizophrenic) psychotic patients, and to a lesser degree patients with less severe types of functional psychiatric disturbance, are not merely deviant from norms and expectancies in society, but are really handicapped in their mental functioning at the fundamental level of controlled information processing. Although therapy is not the primary interest here, some remarks about therapeutic consequences of the topic of this study seem appropriate. No attempt at originality will be made. Acknowledgement of information processing deficits in seriously disturbed psychiatric patients implies acknowledgement of the handicaps of these individuals. Inadequate handling of information is not their sole handicap, of course, other handicaps being altered expectations and self-attitudes, adverse reactions of the social environment, and often also an absence of social support, poor education, and other social disadvantages.

Controlled processing deficiencies render subjects vulnerable to understimulating as well as to overstimulating enviranments. An understimulating environment tends to increase social withdrawal and related behaviors, as demonstrated by the old-fashioned types of mental hospitals, but at the same time withdrawal may represent a kind of protective self-treatment. Patients often actively seek out understimulating social conditions, because they try to avoid overlaad of information. An overstimulating environment is equally problematic. The inability of attentionally handicapped patients to procoss information as fast and efficient as nondisturbed persons may provoke the typical response characteristics of information input overload. In fact many attentional deficits can be interpreted as adjustments to input overload. Miller (1978) has described the general adjustiment 
processes used by living gystems when confronted with information input overlaad. The findings of the present investigation support the asaumption that this is precisely the situation in which many psychotic patienta are more or less continuously placed, in particular when they are involved in highly demanding social interactions. When adjustment to their controlled processing deficiencies fails, breakdown occurs and confusion (psychosis) is the result.

The schizophrenic patient with a fairly severe degree of impairment and vulnerablity is therefore balanced, at the optimum, on a knife-edge (Wing, 1978). The therapeutic implication is a necessity to try to maintain the balance, and therefore to structure the environment, to reduce noxious stimulation, to reduce the need for complex decisions, and to offer the patient some degree of contral over his level of atimulation, but also to prevent maladaptive withdrawal and to ensure an acceptable degree of social interaction. Vulnerable subjects have to learn to live with their handicap, by controlling their level of information input. Insight of the patient can grow over a period of time, with the experience of his vulnerability and of the factors that influence his psychosocial functioning, in a favourable or in an unfavourable sense. But the less capacity an individual has for recognizing his information processing impairments, the more important become the attitudes and behaviors of the people he lives with. They too have to learn to control his level of stimulation. For that reason it is essential that they have some degree of understanding of the nature of the information handling problems theil family member suffers from.

Of course, it is atill more essential that therapists have an understanding of the alleviating or exacerbating influences of environmental conditions. With the growing involvement of various kinds of professionals, like nursing staff, in therapeutic programs it is crucial that they understand and sccept those patients who cannot benefit from therapeutic strategieg that aim at a high level of stimulation and at taking responsibility for one's behavior. An underestimation of the attentional and processing problems that so 
many patients so often encounter in our carefully created therapeutic environments carries the risk of depriving of adequate help those very patients who are most in need of it. It should be realized that their adjustment strategies may be, to some degree, open to change, but not their basic deficits. Rehabilitation in the sense of learning to live with a handicap is a widely practised medical care. Although rehabilitation programs are not lacking, this therapeutic approach deserves more appreciation in psychiatry.

\section{Concluding remarks.}

Besides the therapeutic aspect, there is the ecientific, in particular the taxonomic, aspect, and that aspect is of primary interest in the frame of this investigation. Among the virtues of the level of attentional processes and cerebral information processing is the fact that this level is sufficiently low to be relatively unconfounded by social-interactional influences (although not independent of these processes), and therefore to represent an indication of the truly individual share in the psychiatric problems. On the other hand this level is sufficiently high not to lose sight of the level of mental phenomena, where psychiatric disturbance is identified and on the basis of wich it is traditionally defined. By this position measures of attention and information processing may serve as a bridge between such widely diverging approaches as the study of biochemical brain processes and the study of family and social interactions, that is, between internal and external 'environments'. There is no doubt that prychiatry is in need of unifying conceptions, which can be operationally defined. The present investigation has tried to add to the understanding of the clinical and taxanomic usefulness of this potentially unifying level of analysis.

Especially worth further studying seems the clustering of psychiatric patients and normal subjects using sets of attentional variables, in other words, to apply the methods of numerical taxonomy for the objective classification of subjects. Sets of characters need not be limited to attentional - information processing variables, but will 
more fully approach the aims of polythetic classification when measures of higher levels, and probably also of lower levels of organization, are included. Many research areas in psychiatry will profit from such new classification, from biological psychiatry to social psychiatry. Basing diagnostic decisions, for instance the diagnosis of schizophrenia, on poorly defined or even hardly definable criteria, like the 'precox feeling' of Rünke (1943), although intuitively appealing and widely practised for many years, has definitely become an obsolete approach with the rise of research criteria, the current DSM-III system, and, in the future, fully polythetic classifications. The theoretical foundations of such new empirically derived classifications should not be forgotten (Skinner, 1981). A key challenge for the scientific understanding of abnormal behavior is to achieve a synergism between theory formulation and empirical classification. The basic theoretical position made here is that the recognition of deficits of attention and of information processing in general is of fundamental importance to the understanding of the functional psychatic disorders, and to a lesser degree also of less serious psychiatric disorders. But this is not the wholle story. These deficits are apparently not universal among psychotic patients, while on the other hand (relatively) good functioning subjects may show signs of attentional dysfunctianing.

Even more interesting than clinically severely disturbed (psychotic) patients demonstrating signs of serious attentional dysfunctioning may therefore be those patients who are clinically disturbed to the same degree, but do not show signs of attentional deficits, and those non-psychotic subjects who show signs of serious attentional disturbance. The study of these subjects should enable us to increase our insight in the importance of individual and supra-individual factors related to the vulnerability to develop schizophrenic or ather serious psychiatric disorders, as well as related to the resistance against such disorders. Among the most likely of such 'pratective devices' are level of social competence, certain personality characteristics, and a favourable ecological niche (e.g., Hirsch, 1981; Zubin, 1981). 
Instead of the dichatomous conception of peychosis versus non-psychotic disorders, the continumi conception of paychiatric disturbance should preferably be applied to diagnostic description at the level of clinical phenomena in studies like those advocated here. There are already a number of attempts to measure personality traits related to psychosis and psychotic-like experiences in a continuous way. The Psychoticism scale of Eysenck, included in this investigation, is an example. Other examples are the Depression, Psychasthenia and Schizophrenia scales of the MMPI, the so-called 2-7-8 profile (Gilberstadt and Duker, 1965; Golden and Meeh1, 1979); the Malevolent World ("psychoticism') scale of the Differential Personality Questionnaire (Tellegen, 1978); the Physical Anhedonia and Perceptual Abberration scales of Chapman (Chapman, Chapman and Raulin, 1976; 1978; Chapman, Edell and Chapman, 1980) and the Scales for Psychotic and Psychotic-like Experiences of Chapman (Chapman and Chapman, 1980). These approaches are still far from perfect, which is illustrated, for instance, by the results of Eysenck's Psychoticism scale in the present investigation, but they deserve serious study and ultimately they seem to offer better perspectives than categorical classifications for the description of psychopathology at the clinical-behavioral level.

Because of the firmer theoretical foundation, polythetic classifications based an characters limited to a single, objectively definable level, for instance the level of attention and cerebral information processing, may eventually prove to be more useful in many research areas than classifications based on many levels of analyais (e.g., the 'neurometrics' methodology, developed by John and cowarkers - John, Karmel, Coming, et al., 1977). However, the less behavioral aspects are included in the selected features, the legs the likelihood that the classification will conform to traditional conceptions of psychiatric disturbance, because these are mainly based on the behavioral level. This may prove to limit the clinical usefulness of classifications based on a low level of description, but it does not reduce itsi importance to research in psychiatry. 
These types of research might eventually ciarify such difficult issues as the problem of the persistence of schizophrenia, known to be genetically determined to a relatively high degree, in spite of the adaptive disadvantage of the schizophrenic disarder. A genotype which survives with moderately high frequency cannot be exclusively maladaptive. It has been suggested that the solution concerning schizophrenia may lie in its association with creativity, with a predilection to associative reasoning and unpredictability of respons. (Hammer and Zubin, 1968; Hasenfus and Magaro, 1976). A relation between creativity and psychopathology has been supposed since a long time, of course, and may even be considered part of our cultural heritage. The potential to generate more low probability responses provides an adaptive advantage to individuals characterized by this trait (see, for instance, Lumsden and Wilson, 1981). In the course of his evolution man has developed a very high level of cognitive arganization. The human organism has abandoned many automatic guides of less highly developed organisms, and has to make choices continually at many crucial moments. In a certain sense, schizophrenics could be considered to be in the van of human evolution by virtue of their celatively poorly developed data driven processing and consequently stronger appeal to conceptually driven processing. A maladaptive van, however, in which confusion reigns and real. creativity is smothered. A fascinating area of research opens when the adaptive advantages of information processing characteristics are considered which only at the extremes of their distributions, and eapecially when combined with certain maladaptive learning experiences, manifest themselves as vulnerability, and eventually as (achizophrenic) psychosis. Though this may seem a rather ambitious program, it should be remembered that ultimately "every aspect of thinking can be viewed as a high-level description of a system which, on a low level, is governed by simple, even formal, rules" (D.R. Hofatadter - Gödel, Escher, Bach: An Eternal Golden Braid). 
SUPMMARY

The investigation presented here concerns a study of the relations between psychiatric disturbance, in particular the concept of schizophrenia, and measures of attentional deficit. Current diagnostic practice, although constantly improving, threatens to stagnate by clinging to of ten difficult to define and unreliable descriptions of behavioral, emotional and cognitive deviations from normality. It seems 1 ikely that, at least for research purposes, polythetic classifications, based on the principles of numerical taxonony, will tend to replace traditional diagnostic classifications. A polythetic classification is based upon information from many levels or systems of functioning. One of these levels offering potentially attractive characters to such new classification strategies is the level of attentional processes and cerebral information processing.

After Chapter 1 has introduced the subject of this investigation, Chapter 2.I describes the history of the schizophrenia concept, including recently developed research criteria for this diagnosis. The problem of validation of schizophrenia definitions gets attention. The predictive capacity of various diagnostic systems as well as of symptoms taken individually is discussed, and the poor prognosis conception of schizaphrenia is contrasted with the schizophrenia spectrum concept. 
In Chapter 2.2 the concept of attention is discussed. Modern thearies on this concept consider it to represent the control process in the passage of information through the information processing systems of the central nervous system. Current theories of attention are thus part of theories of cerebral information processing, and defects of attention refer, according to this wiew, to dysfunctions of the (cognitive) information processing systems, but not simply to some particular atage of information processing.

Chapter 2.3 describes attentional oysfunctioning in schizophrenia. Since many years attentional deficits have been recorded in (schizophrenic) psychotic patients. Psychotic symptomatology is increasingly recognized as an adaptation to dysfunctioning attentional and information processing mechanisms. These basic defects in the organization of the central nervous system functions are brought together in the concept of vulnerability.

In Chapter 2.4 some models of schizophrenia, based on dysfunctions of attention and information processing, are discussed, to illustrate the hypothesis-generating power of this approach. Preference is given to the view that schizophrenia, as it is traditionally clinically recognized, is not characterized by some single specific deficit of function. Rather, it seems likely that the psychophysiological basis of schizophrenia lies in the way the central nervous system is organized.

After these intraductory chapters, the aims of the present investigation are farmulated in Chapter 3 . This study aims at exploring the potentialities of some global measures of attention or controlled information processing as objective indicators of psychiatric disturbance, especially those types of disturbance which are clinically recognized as schizophrenia and related disorders (mon-schizophrenic psychosis; schizotypal personality disorder). 
Chapter 5 introduces the diagnostic procedures and the attentional tasks used in the investigation. Patient subjects are classified according to the DMS-III criteria into the diagnostic categaries of neurosis, schizotypal personality disorder, non-schizophrenic psychosis, and schizophrenia. A fifth group consists of normal control subjects. Additional diagnostic classifications are offered by scores on rating scales of the variables affective flattening, social isolation, and premorbid asociality ("schizophrenia variables"), and by the score on the psychaticism scale of the Eysenck Personality Questionnaire. The extraversion, neuraticism and lie scales of this inventory are not included in the hypotheses, but take also part in the exploratory part of the investigation. Measures of attention are: best cycle and total scores of the quality of smooth pursuit eye movements (SPEM), reaction time (mean and redundancy deficit), anission exrars an the continuous performance test (CPT); simple and complex versions (these three tasks constitute the set of performance measures), and an electrophysiological measure, the contingent negative variation (CNV); $C Z$ and $F z$ derivations, early component, late component and post imperative negative variation. The literature on the selected diagnostic and attentional variables is discussed, and the relevance of these variables to the schizophrenia concept is considered.

The hypotheses to be tested in this investigation are presented in Chapter 4. The first hypotheses concern the interrelations between the attentional variables (I) and the interrelations between psychiatric diagnosis and the diagnostic variables (II and III). Intercorrelations within the set of attentional variables are supposed to be pasitive and significant. Positive and significant correlations are also predicted between rank ordered psychiatric diagnoses and diagnostic variables. The central hypothesis (IV) assumes positive correlations between attentional dysfunctioning, as defined by the attentional variables, and degree of psychiatric disturbance, as defined by psychiatric diagnosis, rank ordered according to severity. Chapter 6 describes design and procedures of the investigation, which is cross-sectional in nature. 
The results are presented in Chapter 7 and discussed in Chapter 8. Most of the attentional variables appear to be significantly positively intercorrelated. The post imperative negative variation components of the contingent negative variation, central and frontal derivations, and reaction time redundancy deficit take an exceptional position in this respect, however. Within the set of diagnostic variables psychaticism appears to be associated with severity of psychiatric diagnosis. The 'schizophrenia variables" - affective flattening, social isolation and premorbid asociality - demonstrate a special relevance to the schizophrenia concept. The first hypothesis is thus partly refuted and receivea, superficially, generally support, but the basic structure of the attentional set is non-unitary according to a factor analysis. The second and third hypotheses are supported. The fourth and central. hypothesjs is partily confirmed. Reaction Time Redundancy Deficit and CNV $F_{Z}$ and PINV measures do not show clear associations with rank ordered diagnoses, and SPEM Total plays only a minor role.

Psychoticism as defined by Eysenck's Personality Questionnaire is no aternative to psychiatric diagnosis according to these results, although it may prove to be a useful addition. This scale may be more useful to discrimination within a psychiatric patient group than within a mixed - patient plus normal - group. In the present investigation high psychoticism score appears not associated with attentianal dysfunctioning within diagnostic groups. The consistency of the Dutch psychoticism scale is still uncertain, however.

Among the attentional variables there is one real outlier: reaction time redundancy deficit. Although some interesting associations with other variablee warrant further study, this variable appears independent of psychiatric diagrosis, contrary to resulta reported elsewhere. The best discriminators between less and more psychiatricaliy disturbed subjects are in this investigation: the best cycle scare of the smooth pursuit eye movements, mean (simple) reaction time, the omission scores of the continuous performance test, whether simple or complex version, and the early component of the central contingent negative variation. 
Among the scores of smoath pursuit eye movements the best discrimination between psychiatrically good and poor functioning subjects is attained by the best cycle score, but discrimination is optimized by an index including both best cycle and total scores. Serious dysfunctioning (psychotic) patients seem unable to improve much on their eye tracking performance as reflected in their tatal score, while better patients and normals have an ability to perform better.

While the early component of the central contingent negative variation is the electrophysiological measure showing the highest assaciation with degree of psychiatric disturbance as formalized in psychiatric diagnosis, the difference between central and frontal amplitudes appears also relevant to the discrimination between diagnostic categories. The more disturbed patients seem characterized by low amplitude of the central contingent negative variation in combination with a frontal amplitude wich is high relative to the central one. This result is explained as reflecting differences of activation between central and frontal cortical areas suggesting overactivation of the frontal cortex relative to the central cortex in the clinically more disturbed patients. The reverse applies to the post imperative negative variation. An index is proposed which combines these various observations and maximizes differences on topographical CNV characteristics among diagnostic categories.

At the level of attentional functioning there is no very convincing distinction between schizophrenic (DSM-III) and non-schizophrenic psychotic patients in this investigation, but differences are reflected in electrophyaiological measures and in the scores on affective flattening and premorbid asociality, schizophrenics scoring higher. A two-component model of schizophrenia, as it is traditionally conceptualized, is suggested. The first component is desintegration-proneness or vulnerability, the second component consists of poor social competence skills. Both seem relatively independent. 
The results support the conception of psychiatric disturbance as a continuum of gradually increasing desintegration of mental

functioning. The poorer the attentional performance, the higher the probability the subject belongs to a diagnastic category clinically recognized as psychotic. The borderline character of schizotypal personality disorder is illustrated by its position between psychotic and neurotic patients concerning some attentional variables. The continuum conception is reflected in the results of a hierarchical agglomerative clustering procedure.

Finally some suggestions are made about future research. In particular the use of polythetic classifications is advocated, including attentional variables in its selected variables. In addition, it is suggested that the study of attentional dysfunctioning in non-psychotic patients and normals, and the study of severely disturbed patients not showing signs of attentional deficit, may even be more promising than current applications of these research strategies. 
SAMENUATTING

Het hier gepresenteerde onderzoek omvat een studie naar de relaties tussen psychilatrische stoornissen, in het bijzonder het schizofrenie concept, en maten van aandachtsdysfunkties. Hoewel de klinischdiagnostische praktijk voortdurend aan kwaliteit wint, dreigt deze ontwikkeling toch te stagneren doordat gedrags-, emotionele en cogmitieve afwijkingen van de normaliteit vaak moeilijk en met beperkte betrowwbaarheid te definiëren zijn. Het lijkt waarschijnlijk dat polythetische klassifikaties, gebaseerd op de principes van de numerieke taxonomie, traditionele diagnostische klassifikaties zullen gaan vervangen, in elk geval in het wetenschappelijk onderzoek. Een polythetische klassifikatie is gebaseerd op informaties afkomstig van vele niveaus of systemen van funktioneren. Het niveau waarop zich de aandachtsprocessen en de cerebrale informatieverwerking afspelen lijkt interessante maten te bieden ten behoeve van deze nieuwe klassifikaties.

Hoofdstuk I leidt het onderwerp van dit onderzoek in. Hoofdatuk 2.1 beschrijft vervolgens de geschiedenis en ontwikkeling van het schizofrenie concept, met inbegrip van recente, speciaal voor onderzoeksdoeleinden ontwikkelde criteria voor deze diagnose. Er wordt aandacht geschonken aan het probleem van de validatie van schizofrenie definities, en in dat kader wordt de predictieve validiteit van diverse diagnostische systemen zowel als van individuele symptomen kritisch besproken. Het concept van de 'kern'schizofrenie met slechte prognose 
wordt gesteld tegenover het concept van het schizofrenie spectrum.

In Hoofdstuk 2.2 wordt het aandachtsconcept besproken. Naar huidige opvattingen vertegenwondigt het aandachtsconcept het kontrole-proces tijdem de informatieverwerking door de hersenen. Recente theorieèn betreffende het fenomeen aandacht maken dan ook deel wit van theorieën van de cerebrale informatieverwerking. Aandachtsstoornissen wijzen volgens deze opvattingen op dysfunkties van de (cognitieve) informatieverwerkende systemen, maar niet op defekten die zich beperken tot specifieke en welomschreven stadia van informatieverwerking.

In Hoofdstuk 2.3 worden aandachtsfunktiestoornissen bij schizofrenie behandeld. Aandachtsdefekten bij (schizofreen) psychotische patienten zijn al sinds vele jaren bekend. Er is een tendens om psychotische symptomen te beschowwen als adaptaties aan dysfunktionerende aandachtsprocessen en informatieverwerking. Deze basale defekten in de organisatie van het centrale zenustelsel worden wel ondergebracht in het kwetsbaarheid ("vulnerability") concept.

In Hoofdstuk 2.4 wordt een aantal schizofrenie modelien gepresenteerd die $z i j n$ gebaseerd op stoornissen van de aandacht en informatieverwerking. Deze modellen illustreren de stimulans tot hypothesevorming die van de 'information processing' benadering uitgaat. De voorkeur gaat dezerzijds uit naar de opvatting dat schizofrenie, zoals dat concept traditioneel klinisch wordt gehanteerd, niet wordt gekenmerkt door een enkel specifiek funktiedefekt, maar dat al thans op psychofysiologisch niveau veeleer moet worden gedacht aan algemene arganisatiekenmerken van het centrale zenuwstelsel.

Na deze inleidende hoofdstukken worden de doelstellingen van het onderhavige onderzoek uiteengezet in Hoof dstuk 3. Centraal staat het onderzoeken van de mogelijkheden van een arntal globale aandachtsmaten om te dienen als abjektieve indikatoren van psychisch dysfunktioneren, in het bijzonder van die psychiatrisohe stoornissen die klinisch worden gedefinieerd als schizofrenie en daaraan verwante stoornissen (niet-schizofrene psychose; schizotypische persoonlijkheidsstoornis). 
Hoofdstuk 5 introduceert de diagnostische methoden en de aandachtstesits in dit onderzoek. Patienten worden geklassificeerd volgens de DSM-III criteria in de volgende diagnostische categorieën: neurose, schizotypische persoonlijkheidsstoornis, niet-schizofrene psychose en schizofrenie. Daarnaast neemt een groep normale vrijuilligers aan het onderzoek deel. Naast de psychiatrische diagnose worden middels beoordelingsschalen de variabelen affektieve vervlakking, sociale isolatie en premorbide sociale onaangepastheid ("schizofrenie variabelen") gescoord. Tenslotte participeert ook de score op de psychoticisme schaal van de Eysenck Personality Questionnaire als diagnostische variabele in het onderzoek. De extraversie, neuroticisme en leugen schalen van deze persoonlijkheidsuragenlijst zijn niet opgenomen in de hypothesen, mar wel in het exploratieve deel van het onderzoek. Aandachtsmaten zijn: de kualiteit van de gladde volgbewegingen van de ogen (SPEM, scores voor de beste periode en voor het totaal), de reaktie-tijd (scores voor gemiddelde en voor "redundancy deficit'), en de vals-negatieve reakties bij de 'continuous performance test' (CPT, in een eenvoudige en een gekompliceerde veraie). Deze variabelen varmen de 'performance' maten. Daarnaast een electrofysiologische variabele: de contingente negatieve variatie (CNV, $C Z$ en $F z$ afleidingen), waarvan diverse aspekten warden gemeten (vroege komponent, late komponent en post imperatieve negatieve wariatie). De geselekteerde diagnostische en aandachtevariabelen worden $\mathrm{kritisch}$ besproken. Vooral aan de relevantie van deze variabelen woor het schizofrenie concept wordt aandacht geschonken.

In Moofdstuk 4 worden de hypothesen geformuleerd die in dit onderzoek worden getoetst. Deze hypothesen hebben deels betrakking op de onder 1 inge relaties tussen de aandachtsvariabelen (I) en op de onderijnge relaties tussen psychiatrische diagnose en diagnostische variabelen (II en III). De intercorrelaties binnen de set van aandachtsvariabelen worden verondersteld positief en significant te zijn. Pasitieve en significante correlaties worden ook voorspeld tussen gerangordende psychiatrische diagnosen en diagnostische variabelen. De centrale hypothese (IV) veronderstelt pogitieve correlaties tussen enerzijids de andachtovariabelen, anderzijds de 
naar ernst geordende psychiatrische diagnosen. Hoofdstuk 6 is gewijd aan het ontwerp en de procedures van het onderhavige 'cross-sectional" onderzoek.

De resultaten worden gepresenteerd in Hoofdstuk 7 en de diskussie vindt plaats in Hoofdstuk 8. De meeste aandachtsmaten biljken significant positief te intercorreleren. Uitzonderingen op deze regel zijn echter de post imperatieve negatieve variatie componenten van de contingente negatieve variatie, centrale zovel als de frontale afleidingen, en het reaktie-tijd "redundancy deficit". Binnen de set van diagnostische variabelen blijkt psychoticisme gecorreleerd met ernst van de paychiatrische diagnose. De variabelen affektieve vervlakking, sociale isolatie en premorbide sociale onaangepastheid geven blijk wan een specifieke relevantie voor het schizofrenie concept. De eerste hypothese wordt derhalve gedeeltelijk verworpen en, oppervlakkig beschouwd, in het algemeen bevestigd. Een faktor analyse wan de aandachtsvariabelen wijst evenwel op meerdere 'dimensies' binnen deze set. De tweede en derde hypathese worden bevestigd. De vierde en centrale hypothese wordt gedeeltelijk bevestigd. Reaktie-tijd 'redundancy deficit' en CNW Fz en PINV maten vertonen geen duidelijke relatie met naar ernst geordende diagnosen, en SPEM Totaal speelt in dit opzicht ook een ondergeschikte rol.

De resultaten geven aan dat psychoticisme, zoals gedefinieerd door de Eysenck Personality Questionnaire, geen alternatief biedt voor traditionele psychiatrische diagnostiek, hoewel deze persoonlijkheidsmaat als aanvulling daarop zinvol kan blijken. Het nut van de psychoticisme schal lijkt eerder te liggen in de diskriminatie binnen een populatie van psychiatrische patiênten dan binnen een populatie waarvan ook nietpatienten deel uitmaken. In dit onderzoek werd binnen de diagnostische groepen geen verband gevonden tussen hoogte van de psychoticisme score en kwaliteit van het aandachtsfunktioneren. De consistentie van de Nederlandse psychoticisme schaal is echter vooralsnog discutabel.

Onder de aandachtsvariabelen valt er én duidelijk uit de boot: reaktie-tijd 'redundancy deficit'. Welisuaar zijn er enkele 
Interessante samenhangen met andere variabelen, mar het ontbreken van enig verband met psychiatrische diagnose is in strijd met elders gerapporteerde bevindingen. De volgende aandachtsvariabelen diskrimineren het best tussen minder en meer psychiatrisch gestoorde proefpersonen: de beste-periode-score van de gladde valgbewegingen van de ogen, de gemiddelde reaktie-tijd, de scores woor de vals negatieve reakties bij de continuous performance test, zovel eenvoudige als gekompliceerde versie, en de vroege komponent van de centrale contingente negatieve variatie.

Hoewel van beide gladde volgbewegingescorea de beste-periode-score het best discrimineert tussen in psychiatrisch opzicht goed en slecht funktionerende proefpersonen, verbetert deze diskriminatie bij toepassing van een index die uit zovel de beste-periode-scare als de totale score is samengesteld. Psychiatrisch slecht funktionerende (psychotische) patiënten lijken minder in staat op dit vlak beter te presteren dan tot uiting komt in hun totale score dan beter funktionerende psychiatrische patiënten en niet-patiënten, die wel in staat zijn tot duidelijk betere prestaties.

Van de electrophysiologische maten vertoont de vroege komponent van de centrale contingente negatieve variatie de hoogste samenhang met de ernst van de psychiatrische diagnose. Het amplitude verschil tussen centrale en frontale registraties blijkt echter ook relevant voor de diskriminatie tussen diagnostische categorieën. De meer dysfunktionerende patiënten 1 ijken gekenmerkt door een lage amplitude van de centrale contingente negatieve variatie in kombinatie mot oen in verhouding hoge amplitude van de frontale afleiding. Deze bevinding wijgt op verschillen in aktivatie tusaen de centrale en de frontale cortex, zodanig, dat de klinisch meer dysfunktionerende patiënten gekenmerkt. lijken door overaktivatie van de frontale cortex, in verhouding tot de centrale cortex. Het omgekeerde geldt voor de post imperatieve negatieve variatie. Er wordt een index voorgesteld die deze diverse bevindingen integreert en aldus de diskriminatie tussen de diagnostische groepen ten aanzien van topografische CNV aspekten bevordert. 
Op het niveau van het aandachtsfunktioneren is er geen overtuigend onderscheid tusaen schizofreen (DSM-III) en niet-schizofreen psychotische patièten in dit onderzoek, maar verschillen komen wel tot uiting in de electrophysiologische maten en de score vaor affektieve vervlakking en premorbide sociale onaangepastheid. Schizofrenen scoren hoger op deze variabelen. Er wordt voorgesteld schizofrenie, zoals dat begrip traditioneel wordt opgevat, te interpreteren met behulp van een twee-komponentem model. De eerste komponent is predispositie tot desintegratie ('vulnerability'), de tweede komponent bestaat uit gebrekkige sociale vaardigheden. Beide komponenten lijken relatief onafhankelijk van elkaar.

De resultaten steunen de opvatting dat psychiatrisch dysfunktioneren moet worden opgevat als een continuüm van geleidelijk toenemende desintegratie van het psychisch funktioneren. Hoe slechter de aandachtsprestaties, hoe groter de warsehijnlijkheid dat de proefpersoon in kwestie moet worden geplaatst in een diagnostische kategorie die klinisch wordt gedefinieerd als psychotisch. Het 'borderline' karakter van de schizotypische persoonlijkheidsstoornis komt tot uiting in de plaats die deze kategorie voor wat betreft een aantal aandachtsvariabelen inneemt: tussen psychotische en neurotische patiëntenkategorieën. De continuün conceptie is terug te vinden in de resultaten van een hiërarchische agglomeratieve clusteranalyse.

Tenslotte worden enkele suggesties gedaan ten aanzien van toekomstig onderzoek. In het bijzonder wordt het gebruik van polythetische klassifikaties aanbevolen, die mede gebaseerd zijn op aandachtsvariabelen. Tevens wordt het waridevol geacht aandachtsfunktiestoornisisen van niet-psychotische patiënten en van normale proefpersonen verder te onderzoeken, alsmede onderzoek te verrichten bij die ernstig psychiatrisch giestoorde patiënten die geen relevante aandachtsdefekten vertonen. Deze onderzoeksterreinen kunnen het inzicht in de rol van aandachtsatoornissen bij psychiatrische stoornissen vergroten, en bijdragen aan de kennis van faktoren die bescherming bieden tegen psychische desintegratie. 
APPENDICES

APPENDIX - 1. DIAGNOSTIC CRITERIA.

(1) DSM-III Diagnostic Criteria for Schizophrenia.

A. At least one of the following during a phase of the illness:

(1) Bizarre delusions (content is patently absurd and has no possible basis in fact), such as delusions of being controlled, thought broadcasting, thought insertion, or thought withdrawal.

(2) Somatic, grandiose, religious, nihilistic or ather delusions without persecutory or jealous content.

(3) Delusions with persecutory or jealous content, if accompanied by hallucinations of any type.

(4) Auditory hallucinations in which either a voice keeps up a running commentary on the individual's behavior or thoughts, or two or more voices converse with each other.

(5) Auditory hallucinations on several occasions, with content of more than one or two words having no apparent relation to depression or elation.

(6) Incoherence, marked loosening of associations, markedly illogical thinking or marked poverty of content of speech, if associated with at least one of the following:

(a) blunted, flat or inappropriate affect

(b) delusions or hallucinations 
(c) catatonic or other grossly disorganized behavior.

B. Deterioration from a previous level of functioning in such areas as work, social relations, and self-care.

c. Duration: Continuous signs of the illness for at least six months at some time during the person"s life with some signs of the iliness at present. The six-month period must include an active phase during which there were symptoms from $A$, with or without a prodromal or residual phase, as defined below: Prodromal phase: A clear deterioration in functioning before the active phase of the illness not due to a disturbance in mood or to a Substance Use Disorder, and involving at least two of the gymptoms noted below.

Fesidual phase: Persistence following the active phase of the illness, of at least two of the symptoms noted below, not due to a disturbance in mood or to a Substance Use Disorder. Prodromal or Residual 5ymptoms:

(a) social isolation or withdrawal

(b) maxked impairment in role functioning as wage-earner, student, or homemaker

(c) markedly peculiar behavior (e.g., collecting garbage, talking to self in public, hoarding food)

(d) merked impairment in personal hygiene and grooming

(e) blunted, flat, or inappropriate affect

(f) digressive, vague, overelaborate, circumstantial, or metaphorical speech

(g) odd or bizarre ideation, ar magical thinking, e.g. * superstitiousness, clairvoyance, telepathy, 'sixth sense', 'others can feel my feelings', overvalued ideas, ideas of reference

(h) unusual perceptual experiences, c.9., recurrent illusions, sensing the presence of a force or person not actually present

Examples: Six months of prodromal symptoms with 1 week of symptoms from A; no prodromal symptoms with six months of symptoms from $A$; no prodromal symptoms with two weeks of symptoms from $A$ and six months of residual symptoms; six months 
of symptoms from A, apparently followed by several years of complete remission, with 1 week of symptoms in $A$ in current episode.

D. The full depressive or manic syndrome (criteria $A$ and $B$ of major depressive or manic episode), if present, developed after any psychotic symptoms, or was brief in duration relative to the duration of the psychotic symptoms in $A$.

E. Onset of prodromal or active phase of the illness before age 45.

F. Not due to any Organic Mental Disorder or Mental Retardation.

Added in this investigation:

Psychotic symptoms (delusions, hallucinations, incoherence or marked loosening of associations, or behavior that is grossly disorganized or catatonic) have been present during the last week before testing.

(2) Diaqnostic criteria for a Non-Schizophrenic Psychotic Disorder DSM-111.

This diagnostic category corresponds with the DSM-III categories of Paranoid Disorders, Schizoaffective Disorder, Schizophreniform Disorder, Brief Reactive Psychosis and Atypical Psychosis. Subjects in this category at least meet the following criteria:

(1) The presence during a period of at least one week of psychatic symptoms (delusions, hallucinations, incoherence or marked loosening of associations, or behavior that is grossly disorganized or catatonic).

(2) Psychotic symptoms have been present during the last week before testing.

(3) Not meeting the criteria for a diagnosis of Schizophrenic Disorder according to DSM-III. 
(3) Diagnostic criteria for Schizotypal Personality Disorder DSM-III.

The following are characteristic of the individual"s current and long-term functioning, are not limited to episodes of illness, and cause either significant impairment in social or occupational functioning or subjective distress.

\section{A. At least four of the following:}

(1) Magical thinking, e.g., superstitiousness, clairvoyance, telepathy, 'sixth sense', 'others can feel my feelings", (in children and adolescents, bizarre fantasies or preoccupations).

(2) Ideas of reference.

(3) Social isolation, e.g., no close friends or confidants, social contacts limited to essential everyday tasks.

(4) Recurrent illusions, sensing the presence of a force or person not actwally present (e.g., 'I felt as if my dead mother were in the room with me'), depersonalization, or derealization not associated with panic attacks.

(5) Odd speech (without loosening of associations or incoherence), e.g., speech that is digressive, vague, overelaborate, circumstantial, metaphorical.

(6) Inadequate rapport in face-to-face interaction due to constricted or inappropriate affect, e.g., aloof, cold.

(7) Suspiciousness or paranoid ideation.

(B) Undue social anxiety or hypersensitivity to real or imagined eriticism.

B. Does not meet the criteria for Schizophrenia.

Added in this investigation:

(1) Never had a clear poychotic episode.

(2) Also qualifying for a 'neurotic' disorder as defined in (4). 
(4) Diagnastic criteria for a "Neurotic" Disorder - DSM-III.

This diagnostic category corresponds with the DSM-III categories of Dysthymic Disorder, Anxiety Disorders, Somatoform Disorders and Dissociative Disorders. (Although DSM-III also includes the diagnostic class of Psychosexual Disorders in the 'Neurotic' Disorders, subjects meeting only these criteria were not included in the present investigation).

\section{Added in this investigation:}

(a) Not qualifying for the diagnosis of Schizotypal Personality Disorder.

(b) Never had a clear psychatic episode. 
APPENDIX - 2. BATING SCALE FOR EMOTIONAL BLUNTING

(Abrans and Taylor, 1978).

Item

Rating

Affect

1. Absent, shallow, incongruous mood

2. Constricted affect (narrow range)

$\begin{array}{lll}0 & 1 & 2\end{array}$

3. Unvarying affect (lacks modulation)

0.12

4. Unrelated affect (lacks warmth, empathy)

$\begin{array}{lll}0 & 1 & 2\end{array}$

$\begin{array}{lll}0 & 1 & 2\end{array}$

Behavior

5. Expressionless face

$0 \quad 12$

6. Unwarying, monotonous voice

$\begin{array}{lll}0 & 1 & 2\end{array}$

7. Seclusive/withdrawn, awoids social contact

$0 \quad 12$

8. Lacks social graces (negligent dress, ill-mannered, unbathed)

9. Difficult to excite emotions/unresponsive

10. Lacke spontaneity

$0 \begin{array}{lll}0 & 1 & 2\end{array}$

11. Causeless, silly laughter/silly disposition

$0 \begin{array}{lll}0 & 1 & 2\end{array}$

12. Indifferent to surroundings (staff, visitors, patients, physical environs)

012

D 12

Thought content

13. Indifference/lack of affection for family, friends $0 \quad 12$

14. Indifference/unconcern for oun present situation $\quad 0 \quad 12$

15. Indifference/unconcern for oun future (lacks plans, ambition, desires, drive) 0.12

16. Paucity of thought (unable to elaborate on answers) $\begin{array}{lll}0 & 1 & 2\end{array}$

Total score:

$0=$ absent, 1 = slight or doubtful, 2 = clearly present. 
APPENDIX - 3. SCHEDULE FQR AFFECTIVE DISORDERS AND SCHIZOPHRENIA.

Section: Social functioning.

(Spitzer and Endicott, 1978).

Social relations. Best level of social relations during the last 5 years that lasted at least several months. Consider contact that has a pleasurable quality and is not merely for the purpose of completing some task or fulfilling a duty. May include farmily members not living in the same household.

During the past 5 years, when was the period you had the most to do with other people socially? How much did you have to do socially with friends or with other people then?

(What about visiting or having people over to your place?)

(What about church activities, bowling, playing cards, ete.?)

(Whom do you see?)

(How close are you to them?)

(Would you consider any of them close friends - somebody you could really trust?)
0. No information or not shure.

1. Superior, e.g., had many special friends that he saw regularly and frequently and was very close to.

2. Very good, e.g., had several special friends that he saw regularly and frequently and was close to.

3. Good, e.g*, had 1 or 2 special friends that he saw from time to time and was fairly close to.

4. Fair, e.g., had only 1 special friend that he saw from time to time and was fairly close to OR social contacts were limited to friends or acquaintances that he was not very close to emotional1y.

5. Poor, e.g., had no specilal friends that he saw from time to time and was fairly close to, oR social contacts were 1 imited to a small number of friends or acquaintances that he was not very close to.

6. Very poor, e.g., had no special friends and social contacts were limited to 1 or 2 people that he was not very close to emotionally.

7. Grossly inadequate, e.g., had practically no oocial contact. 
APPENDIX - 4. PREMORBID ASOCIAL ADJUSTMENT SCALE.

(Gittelman-Klein ${ }^{2}$ in: Kokes, Strauss and Klorman, 1977)

Sixth year to adolescence (6th year through 13 th year):

1. Isolation or active socially

0 Not withdrawn, active social interaction

1

2

3

4 Moderately withdrawn, given to daydreaming and excessive fantasy 5

6 Unrelated, withdrawn, and isolated.

2. Peer relationships

o Many friends, close relationships

1

2 Casual friends only

3

4 Deviant friendship patterns: Friendly with children older or younger only, or relatives only, or with a single or a couple of 5 individuals only

6 Social isolate, no friends.

3. Intereste

0 Active - interested in a variety of school and social activities and hobbies

1

2

3

4 Introverted interests - one or a few hobbies which require no contact with others (i.e., stamp collection, reading, moviegoing school work

5

6 No interests - withdrawn and indifferent toward interests of the average youngster (no hobbies, no participation in sports, mo deep interest of any kind).

Adoleacence:

4. Isolation

0 No withdrawn, active social interaction

1

2

3

4 Moderately withdrawn, given to daydreaming and excessive fantasy 5

6 Unrelated, withdrawn, and iaolated. 


\section{Peer relationships}

0 Many friends - with a few close relationships

1

2 Casual friends anly

3

4 Deviant friendship patterns: friendly with children older or younger only, or relatives only, or with a single or a couple of 5 individuals only

6 Social isolate, no friends.

6. Interest

0 Active - interested in a variety of school and social activities and hobbies

1

2

3

4 Introverted interests - one or a few hobbies which require no contact with others (i.e., stamp collection, reading, moviegoing, school work) 5

6 No interests - withdrawn and indifferent toward interests of the average youngster (no hobbies, no participation in sports, no deep interest of any kind).

7. Sociosexual adjustment (from 16 to 20 years of age) (Ignore behavior before the age of 16)

The descriptive statements are given as guidelines and are not comprehensive. For behavior not listed, ratings should be made using the scales as a continutum from good, normal adjustment, to extremely poor, to no sexual drive.

0 Healthy interest in girls/boys, steady close relationship with sexual intercourse or sexual play

Went out with girls/boys regularly, steady close relationship with little or no sexual play

1 Went out with girls/boys regularly, steady casual relationship with or without sexual play or intercourse

2 Went out with girls/boys regularly, passing casual relationship with or without sexual intercourse

3 Casual occasional contact with girls/boys with or without sexual intercourse or sexual play

4 Interested in girls/bays, but never went out on dates

5 Homosexual involvement only

6 No sexual interests in either sex 


\section{Psychoticism Scale:}

1. Do you stop to think things over before doing anything?

2. Would you being in debt worry you?

3. Do you lock up your house carefully at night?

4. Would it upset you a lot to see a child or an animal suffer? (No)

5. Do you believe insurance schemes are a good idea?

6. Would you take drugs which may have strange or dangerous effects?

7. Do you enjoy hurting people you love?

B. Do you have enemies who want to harm you?

9. Do you enjoy practical jokes that can sometimes really hurt people?

10. Do good manners and cleanliness matter much to you?

11. Do you think marriage is old-fashioned and should be done away with?

12. Do people who drive carefully annoy you?

13. Do most things taste the same to you?

14. Does it worry you if you know there are mistakes in your work?

15. Do you like to arrjue at appointments in plenty of time?

16. Is (or was) your mother a good woman?

17. Are there several people who keep trying to avaid you?

18. Do you think people spend too much time safeguarding their future with savings and insurances?

19. Do you try not to be rude to people?

20. When you catch a train do you often arrive at the last minute?

21. Do your friendships break up easily without it being your fault?

22. Do you sometimes like teasing animals?

23. Would you like other people to be afraid of you?

24. Do people tell you a lot of lies?

25. Would you feel very sorry for an animal caught in a trap? 


\section{Extraversion Scale:}

1. Do you have many different hobbies?

(Yes)

2. Are you a talkative person?

3. Are you rather lively?

4. Can you usually let yourself go and enjoy yourself at a lively party?

5. Do you enjoy meeting new people?

6. Do you tend to keep in the background on social occasions? (No)

7. Do you like going out a lot?

8. Do you prefer reading to meeting people?

9. Do you have many friends?

10. Would you call yourself happy-go-lucky?

11. Do you usually take the initiative in making new friends?

12. Are you mostly quiet when you are with other people?

13. Can you easily get some life into a rather dull party?

14. Do you like telling jokes and funny stories to your friends? (Yes)

15. Do you like mixing with people?

16. Do you nearly always have a "ready answer" when people talk to you?

17. Do you like doing things in which you have to act quickly? (Yes)

18. Do you often take on more activities than you have time for? (Yes)

19. Can you get a party going?

20. Do you like plenty of bustle and excitement around you?

21. Do other people think of you as being very lively?

\section{Neuroticism Scale:}

1. Does your mood often go up and down?

2. Do you ever feel 'just miserable' for no reasan?

3. Do you often worry about things you should not have done or said?

4. Are you an irritable person?

5. Are your feelings easily hurt?

6. Do you often feel 'fed-up'?

7. Are you often troubled about feelinge of guilt?

8. Would you call yourself a nervous person? 
9. Are you a worrier?

10. Do you vorry about awful things that might happen?

11. Would you call yourself tense or 'highly-strung'?

12. Do you worry about your health?

13. Do you suffer from sleeplessness?

14. Have you often felt listless and tired for no reason?

15. Do you often feel life is very dull?

16. Do you worry a lot about your looks?

17. Have you ever wished that you were dead?

18. Do you worry too long after an embarrassing experience?

19. Do you auffer from 'nerves'?

20. Do you often feel lonely?

21. Are you easily hurt when people find fault with you or the work you do?

22. Are you sometimes bubbling over with energy and sometimes very sluggish?

23. Are you touchy about some things?

\section{Lie Scale:}

1. Have you ever taken the praise for something you knew someone else had really done?

(No)

2. Were you ever greedy by helping yourself to more than your share of anything?

3. If you say you will do something, do you always keep your promise no matter how inconvenient it might be?

4. Have you ever blamed someone for doing sometining you knew was really your fault?

5. Are all your habits good and desirable ones?

6. Have you ever taken anything (even a pin or button) that belonged to someone else?

7. Do you sometimes talk about things you know nathing about?

B. As a child did you do as you were told immediately and without grumbling?

9. Have you ever broken or lost something belonging to someone else?

10. Do you sometimes boast a little? 
11. Have you ever said anything bad or nast about anyone?

12. As a child were you ever cheeky to your parents?

13. Do you always wash before a meal?

14. Have you ever cheated at a game?

15. Have you ever taken advantage of someone?

16. Would you dodge paying taxes if you vere shure you could never be found out?

17. Have you ever insisted on having your oun way?

18. Do you always practice what you preach?

19. Have you ever been late for an appointment or work?

20. Do you sometimes put off until tomorrow what you ought to do today?

21. Are you always willing to admit it when you have made a mistake? 


\section{APPENDIX - 6. CONT INULUS PERFORMANCE TEST.}

$\begin{array}{llllllllllllllllllll}4 & 3 & 9 & 5 & 6 & 7 & 1 & 8 & 1 & 7 & 9 & 1 & 7 & 3 & 1 & 2 & 3 & 4 & 2 & 8\end{array}$ $\begin{array}{llllllllllllllllllll}1 & 9 & 1 & 3 & 2 & 8 & 6 & 9 & 2 & 5 & 7 & 8 & 2 & 4 & 3 & 6 & 7 & 1 & 8 & 5\end{array}$ $\begin{array}{llllllllllllllllllll}8 & 8 & 2 & 3 & 2 & 4 & 1 & 1 & 7 & 6 & 2 & 6 & 8 & 9 & 1 & 6 & 3 & 5 & 5 & 3\end{array}$ $\begin{array}{llllllllllllllllllll}2 & 2 & 1 & 1 & 4 & 5 & 7 & 6 & 2 & 3 & 1 & 9 & 2 & 5 & 7 & 9 & 1 & 9 & 7 & 1\end{array}$ $\begin{array}{llllllllllllllllllll}4 & 1 & 5 & 8 & 7 & 1 & 3 & 5 & 5 & 8 & 3 & 2 & 2 & 9 & 1 & 4 & 1 & 5 & 4 & 9\end{array}$ $\begin{array}{llllllllllllllllllll}4 & 8 & 4 & 9 & 6 & 1 & 9 & 1 & 2 & 3 & 4 & 8 & 4 & 7 & 8 & 7 & 1 & 4 & 5 & 2\end{array}$ $\begin{array}{llllllllllllllllllll}7 & 8 & 1 & 2 & 5 & 5 & 9 & 1 & 7 & 7 & 3 & 5 & 3 & 3 & 5 & 7 & 1 & 2 & 9 & 1\end{array}$ $\begin{array}{llllllllllllllllllll}1 & 9 & 1 & 2 & 4 & 4 & 7 & 1 & 2 & 5 & 5 & 8 & 7 & 1 & 5 & 1 & 9 & 6 & 4 & 7\end{array}$ $\begin{array}{llllllllllllllllllll}6 & 2 & 3 & 5 & 6 & 4 & 2 & 3 & 7 & 1 & 2 & 2 & 1 & 4 & 1 & 1 & 9 & 7 & 1 & 8\end{array}$ $\begin{array}{llllllllllllllllllll}5 & 4 & 9 & 1 & 4 & 1 & 7 & 3 & 2 & 6 & 7 & 1 & 2 & 6 & 5 & 7 & 3 & 9 & 3 & 6\end{array}$ $\begin{array}{llllllllllllllllllll}7 & 1 & 9 & 9 & 5 & 3 & 1 & 7 & 3 & 5 & 8 & 3 & 4 & 6 & 4 & 9 & 1 & 1 & 8 & 2\end{array}$ $\begin{array}{llllllllllllllllllll}6 & 2 & 2 & 6 & 5 & 9 & 1 & 6 & 1 & 3 & 1 & 5 & 9 & 1 & 4 & 7 & 6 & 8 & 1 & 6\end{array}$ $\begin{array}{llllllllllllllllllll}7 & 1 & 9 & 9 & 6 & 4 & 6 & 9 & 7 & 1 & 8 & 8 & 2 & 1 & 6 & 1 & 9 & 2 & 3 & 3\end{array}$ $\begin{array}{llllllllllllllllllll}8 & 4 & 4 & 4 & 5 & 1 & 1 & 8 & 8 & 4 & 6 & 7 & 6 & 8 & 3 & 7 & 1 & 3 & 6 & 2\end{array}$ $\begin{array}{llllllllllllllllllll}6 & 3 & 9 & 1 & 1 & 4 & 7 & 1 & 2 & 2 & 8 & 2 & 8 & 4 & 7 & 3 & 9 & 1 & 1 & 3\end{array}$ $\begin{array}{llllllllllllllllllll}7 & 1 & 5 & 6 & 3 & 2 & 6 & 4 & 8 & 7 & 3 & 6 & 6 & 4 & 8 & 3 & 1 & 8 & 7 & 1\end{array}$ $\begin{array}{llllllllllllllllllll}1 & 1 & 3 & 8 & 4 & 5 & 6 & 3 & 3 & 8 & 5 & 4 & 7 & 3 & 6 & 5 & 5 & 3 & 9 & 1\end{array}$ $\begin{array}{llllllllllllllllllll}4 & 1 & 6 & 8 & 8 & 2 & 4 & 9 & 1 & 8 & 8 & 3 & 6 & 4 & 7 & 4 & 2 & 4 & 3 & 9\end{array}$ $\begin{array}{llllllllllllllllllll}2 & 9 & 1 & 9 & 3 & 9 & 7 & 1 & 4 & 3 & 5 & 5 & 4 & 7 & 2 & 9 & 8 & 9 & 1 & 4\end{array}$ $\begin{array}{llllllllllllllllllll}9 & 1 & 8 & 9 & 2 & 5 & 4 & 5 & 6 & 1 & 7 & 4 & 1 & 3 & 6 & 1 & 1 & 1 & 9 & 2\end{array}$ $\begin{array}{llllllllllllllllllll}1 & 7 & 1 & 8 & 6 & 5 & 8 & 1 & 1 & 8 & 2 & 7 & 1 & 6 & 9 & 1 & 5 & 2 & 8 & 9\end{array}$ $\begin{array}{llllllllllllllllllll}6 & 4 & 8 & 2 & 6 & 4 & 5 & 1 & 1 & 3 & 9 & 1 & 6 & 5 & 2 & 3 & 5 & 7 & 1 & 6\end{array}$ $\begin{array}{llllllllllllllllllll}1 & 1 & 5 & 8 & 7 & 9 & 1 & 6 & 5 & 2 & 2 & 8 & 6 & 1 & 7 & 1 & 7 & 3 & 4 & 5\end{array}$ $\begin{array}{llllllllllllllllllll}9 & 8 & 5 & 5 & 8 & 6 & 9 & 1 & 6 & 6 & 4 & 3 & 2 & 8 & 9 & 9 & 1 & 1 & 7 & 1\end{array}$ $\begin{array}{llllllllllllllllllll}5 & 1 & 6 & 4 & 5 & 7 & 8 & 1 & 3 & 1 & 5 & 3 & 4 & 7 & 1 & 2 & 9 & 5 & 8 & 6\end{array}$

Simple Version : oritical stimuli are 7 and 9. Complex Version: critical stimuli are 7 -followed-by-1 and 9-followed-by-1. 
APPENDIX - 7. REACTION TIME TASK.

Series of regular and irregular reaction time trials. The numbers denote the PI durations. Intertrial-intervals are $4.5 \mathrm{sec}$, interseries-interwals are $15 \mathrm{sec}$. The first trial of the irregular series (' 8 ') is excluded from the final calculations.

$\begin{array}{llllllllll} & 4 & 4 & 4 & 4 & 4 & 4 & 4 & 4 & 4 \\ & 2 & 2 & 2 & 2 & 2 & 2 & 2 & 2 & 2 \\ & 8 & 8 & 8 & 8 & 8 & 8 & 8 & 8 & 8 \\ 8 & 4 & 8 & 4 & 4 & 8 & 8 & 2 & 2 & 8 \\ 8 & 4 & 4 & 2 & 2 & 8 & 2 & 2 & 4 \\ 4 & 2 & 4 & 2 & 8 & 2 & 4 & 8 & 8\end{array}$


List of oral neuroleptic drugs, with equivalent doses in relation to haloperidol.
List of depot meuroleptic drugs with equivalent doses in relati to orel haloperidol.
Haloperidol

Trifluperidol

Benperido1

Pimozide

Thioproperazine

Flupenthixol

Thiothixene

Fluphenazine

Trifluoperazine

Methylperidol

Butyrylperazine

Butaperazine

Thiopropazine

Piperacetazine

Perphenazine

Prochlorperazine

Periciazine

Acetophenazine

Molindone

Carphenazine

Trifluopromazine

Tetrabenazine

Mesoridazine

Dixyracine

Clopenthixol

Chlorpromazine

Chlorprothixene

Levopromazine

Prothipendy 1

Perazine

Thioridazine

Pipamperone

Sulpíride

Promazine
1 Fluspirilene

1 Perphenazine enanthate

1 Flupenthixol decanoate

1 Penfluridol (oral)

1 Fluphenazine decanoate

1 Clopenthixol decanoate

1 Pipothiazine palmitate

2 Pipothiazine undecylenate

3

3

5

5

5

5

5

10

10

10

10

10

20

20

30

30

30

50

60

60

60

70

70

70

100

100 


$$
\begin{aligned}
& \text { All tables: }+=p<.10 \\
& *=p<.05 \\
& * *=p<.01
\end{aligned}
$$

\begin{tabular}{|c|c|c|c|c|c|c|c|c|}
\hline SPEM Total & - & & & & & & & \\
\hline SPEM Best Cycle & $.87^{* * *}$ & - & & & & & & \\
\hline RT Mean & $.62^{* *}$ & $.55^{* * *}$ & - & & & & & \\
\hline RT Red.Def. & -.19 & -.15 & .19 & - & & & & \\
\hline CPT Simple Dm. & .23 & .29 & $.34^{+}$ & .02 & - & & & \\
\hline CPT Simple Com. & .16 & .02 & .17 & .00 & $.35^{+}$ & - & & \\
\hline CPT Compl. Om. & .29 & $.36^{+}$ & $.47^{*}$ & -.13 & $.77^{* *}$ & $.32^{+}$ & - & \\
\hline \multirow[t]{2}{*}{ CPT Compl. Com. } & -.17 & -.17 & .03 & .07 & $.39^{*}$ & $.65^{* * *}$ & .21 & - \\
\hline & $\begin{array}{l}\text { SPEM } \\
\text { Total }\end{array}$ & $\begin{array}{l}\text { SPEM } \\
\text { Best } \\
\text { Cycle }\end{array}$ & $\begin{array}{l}\mathrm{RT} \\
\text { Mean }\end{array}$ & $\begin{array}{l}\text { RT } \\
\text { Red. } \\
\text { Def. }\end{array}$ & $\begin{array}{l}\text { CPT } \\
\text { Simple } \\
\text { Dm. }\end{array}$ & $\begin{array}{l}\text { CPT } \\
\text { Simple } \\
\text { Com. }\end{array}$ & $\begin{array}{l}\text { CPT } \\
\text { Compl. } \\
\text { Dm. }\end{array}$ & $\begin{array}{l}\text { CPT } \\
\text { Compl. } \\
\text { Com. }\end{array}$ \\
\hline
\end{tabular}

Iable 3a. Spearman Correlation Coefficients.

\section{Diagnostic Group: schizophrenia.}


Table 3b. Spearman Correlation Coefficients.

\section{Diagnostic Group: non-schizophrenic psychosis.}

SPEM Total

SPEM Best Cycle $.78^{* *}-$

RTT Mean $\quad-.07 \quad .03$

RT Red.Def. $\quad .12 \quad .15 \quad-.26$

CPT Simple Om. $\quad .03 \quad .17 \quad .46^{*} \quad-.24$

CPT Simple Com. $\quad .13 \quad .29 \quad .50^{*} \quad-.03 \quad .53^{* *} \quad-$

CPT Compl. Om. $\quad .11 \quad .16 \quad .59^{* *}-.37^{+} \quad .81^{* *} .42^{*} \quad-$

$\begin{array}{llllllll}\text { CPT Compl. Com. } & .17 & .04 & .52^{* *} & .04 & .42^{*} & .18 & .55^{* *}\end{array}$

SPEM SPEM RT RT CPT CPT CPT CPT

Total Best Mean Red. Simple Simple Compl. Compl.

Cycle Def. Om. Com. Dm. Com.

Table $3 c$. Spearman Correlation Coefficients.

Diagnostic Group: schizotypal personality disorder.

SPEM Total

SPEM Best Cycle $.78^{* * *}-$

RT Mean

$.33^{+} \quad .29 \quad-$

RT Red.Def.

$.14 \quad .38^{*} \quad .01 \quad-$

CPT Simple 0m. $\quad .36^{+} \quad .49^{*} \quad .37^{+} \quad .29$

$\begin{array}{llllllll}\text { CPT Simple Com. } & .28 & .10 & -.14 & .10 & .34^{+} & -\end{array}$

CPT Compl. Om. $\quad .33^{+} \quad .41^{*} \quad .60^{* *} \quad .11 \quad .76^{* *} \quad .20$

CPT Compl. Com. $.18 \quad .04 . \quad .14 \quad .14 \quad .55^{* *} \quad .46^{*} \quad .63^{* *}$ -

SPEM SPEM RT RT CPT CPT CPT CPT

Tatal Best Mean Red. Simple Simple Compl. Compl.

Cycle Def. Om. Com. Om. Com. 
Table 3d. Spearman Correlation Coefficients. Diagnostic Group: neurosis.

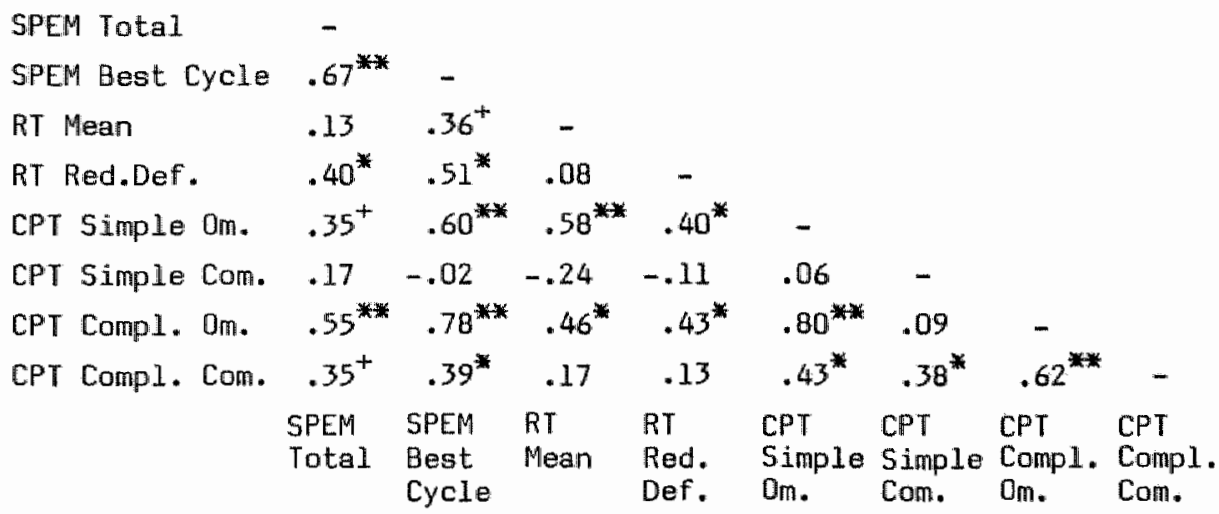

Table 3e. Spearman Correlation Coefficients.

Diagnostic Group: normal.

\begin{tabular}{|c|c|c|c|c|c|c|c|c|}
\hline SPEM Total & - & & & & & & & \\
\hline SPEM Best Cycle & $.61^{* * *}$ & - & & & & & & \\
\hline RT Mean & .28 & $.42^{*}$ & - & & & & & \\
\hline RT Red.Def. & $-.51^{*}$ & $-.33^{+}$ & .00 & - & & & & \\
\hline CPT Simple Om. & -.26 & .04 & .05 & .08 & - & & & \\
\hline CPT Simple Com. & .16 & $.40^{*}$ & .21 & -.12 & $.37^{+}$ & - & & \\
\hline CPT Comp1. Om. & $.36^{+}$ & .23 & .25 & -.17 & .15 & $.36^{+}$ & - & \\
\hline \multirow[t]{2}{*}{ CPT Compl. Com. } & .10 & .25 & $.31^{+}$ & .10 & -.03 & $.33^{+}$ & $.38^{*}$ & - \\
\hline & $\begin{array}{l}\text { SPEM } \\
\text { Total }\end{array}$ & $\begin{array}{l}\text { SPEM } \\
\text { Best } \\
\text { Cycle }\end{array}$ & $\begin{array}{l}\text { RT } \\
\text { Mean }\end{array}$ & $\begin{array}{l}\text { RT } \\
\text { Red. } \\
\text { Def. }\end{array}$ & $\begin{array}{l}\text { CPT } \\
\text { Simple } \\
0_{\mathrm{m} .}\end{array}$ & $\begin{array}{l}\text { CPT } \\
5 \text { imple } \\
\text { Com. }\end{array}$ & $\begin{array}{l}\text { CP T } \\
\text { Compl. } \\
\text { Om. }\end{array}$ & $\begin{array}{l}\text { CPT } \\
\text { Compl. } \\
\text { Com. }\end{array}$ \\
\hline
\end{tabular}


Table 4a. Spearman Correlation Coefficients.

Diagnostic Group: schizophrenia.

CNU CZ CNU CZ CNZ FZ CNV FZ PINU CZ PINU FZ Early Late Early Late

Comp. Comp. Comp. Comp.

$\begin{array}{lcccccc}\text { SPEM Total } & .45^{*} & .31^{*} & .38^{*} & .31^{*} & .49^{*} & .38^{*} \\ \text { SPEM Best Cycle } & .55^{* *} & .43^{*} & .48^{*} & .40^{*} & .51^{*} & .45^{*} \\ \text { RT Mean } & .53^{* *} & .40^{*} & .43^{*} & .27 & .67^{* *} & .53^{* *} \\ \text { RT Red.Def. } & -.09 & -.030 & -.15 & -.33^{+} & .00 & -.08 \\ \text { CPT Simple Om. } & .10 & .02 & .20 & .02 & .21 & .24 \\ \text { CPT Simple Con. } & .11 & .00 & .27 & .11 & .42^{*} & .46^{*} \\ \text { CPT Compl. Om. } & .42^{*} & .31^{+} & .57^{* *} & .35^{*} & .38^{+} & .48^{*} \\ \text { CPT Compl. Com. } & -.07 & -.03 & .03 & -.07 & .27 & .32^{+}\end{array}$

Table 4b. Spearman Correlation Coefficients.

Diagnostic Group: non-schizophrenic psychosis.

$\begin{array}{lcccccc} & \begin{array}{l}\text { CNV Cz } \\ \text { Early } \\ \text { Comp. }\end{array} & \begin{array}{l}\text { CNV Cz } \\ \text { Late }\end{array} & \begin{array}{l}\text { CNU Fz } \\ \text { Early }\end{array} & \begin{array}{l}\text { CNV Fz } \\ \text { Late }\end{array} & \text { PINU Cz PINU Fz } \\ \text { Comp. } & \text { Comp. } & & \\ \text { SPEM Total } & -.10 & -.13 & -.16 & .05 & -.11 & -.27 \\ \text { SPEM Best Cycle } & -.04 & -.01 & .09 & .31^{+} & .05 & .10 \\ \text { RT Mean } & .05 & .18 & -.10 & .22 & .23 & -.11 \\ \text { RT Red.Def. } & .02 & -.09 & .07 & .07 & -.19 & .01 \\ \text { CPT Simple Om. } & .12 & .15 & -.08 & .20 & .25 & -.06 \\ \text { CPT Simple Com. } & .23 & .17 & .10 & .27 & .48^{*} & .25 \\ \text { CPT Compl. Om. } & .15 & .14 & .04 & .23 & .17 & -.20 \\ \text { CPT Compl. Com. } & -.29 & -.16 & -.36^{+} & -.12 & -.11 & -.50^{*}\end{array}$


Iable 4c. Spearman Correlation Coefficients.

Diagnostic Group: schizotypal personality disorder.

$\begin{array}{lllll}\text { CNU CZ } & \text { CNV CZ } & \text { CNU Fz } & \text { CNU Fz } & \text { PINU Cz PINU Fz } \\ \text { Early } & \text { Late } & \text { Early } & \text { Late } & \\ \text { Comp. } & \text { Comp. } & \text { Comp. } & \text { Comp. } & \end{array}$

$\begin{array}{lrrrrrr}\text { SPEM Total } & -.22 & .26 & .01 & .24 & -.11 & .18 \\ \text { SPEM Best Cycle }-.04 & -.04 & .06 & -.18 & -.27 & -.04 \\ \begin{array}{l}\text { RT Mean } \\ \text { RT }\end{array} & .13 & .28 & .28 & .34^{+} & -.33^{+} & .04 \\ \text { RT Red.Def. } & -.25 & -.28 & .07 & -.17 & -.09 & .17 \\ \text { CPT Simple 0m. } & .03 & -.14 & .20 & -.02 & -.57^{* *} & -.04 \\ \text { CPT Simple Com. }-.26 & -.05 & .19 & .20 & -.27 & .10 \\ \text { CPT Compl. Om. } & .15 & -.07 & .24 & -.07 & -.62^{* *} & -.22 \\ \text { CPT Compl. Com. }-. .23 & -.03 & .00 & .07 & -.57^{* *} & -.37^{*}\end{array}$

Table 4d. Spearman Correlation Coefficients.

Diagnostic Group: neurosis.

$\begin{array}{lcccccc} & \text { CNV CZ } & \text { CNV CZ } & \text { CNV Fz } & \text { CNV Fz } & \text { PINU Cz PINV Fz } \\ & \text { Early } & \text { Late } & \text { Carly } & \text { Late } & & \\ & \text { Comp. } & \text { Comp. } & \text { Comp. } & \text { Comp. } & & \\ \text { SPEM Total } & .31^{+} & .18 & .19 & .06 & .06 & .33^{+} \\ \text {SPEM Best Cycle } & .28 & .07 & .15 & -.20 & .04 & .18 \\ \text { RT Mean } & .54^{* *} & .37^{+} & 25 & -.02 & .34^{+} & .04 \\ \text { RT Red.Def. } & .35^{+} & .31^{+} & .40^{*} & .37^{+} & -.11 & .29 \\ \text { CPT Simple Om. } & .26 & .16 & .10 & -.04 & .13 & .19 \\ \text { CPT Simple Com. } & -.27 & -.38^{+} & -.18 & -.23 & .26 & .17 \\ \text { CPT Comp1. Dm. } & .28 & .12 & .21 & -.16 & -.08 & .12 \\ \text { CPT Compl. Com. } & .12 & .11 & -.02 & -.04 & .30 & .29\end{array}$


Iable 4e. Spearman Correlation Coefficients.

Diagnostic Group: normal.

$\begin{array}{llllll}\text { CNU Cz } & \text { CNU } C Z & \text { CNV Fz } & \text { CNV Fz } & \text { PINU Cz } & \text { PINV Fz } \\ \text { Early } & \text { Late } & \text { Early } & \text { Late } & & \\ \text { Comp. } & \text { Comp. } & \text { Comp. } & \text { Comp. } & & \end{array}$

$\begin{array}{lcccccr}\text { SPEM Total } & .45^{*} & .46^{*} & .39^{*} & .39^{*} & .14 & .41^{*} \\ \text { SPEM Best Cycle } & .13 & .09 & .02 & -.03 & .03 & -.01 \\ \text { RT Mean } & .38^{*} & .46^{*} & .26 & .33^{*} & .40^{*} & .29 \\ \text { RT Red.Def. } & -.06 & -.32^{*} & -.11 & -.41^{*} & -.08 & -.29 \\ \text { CPT Simple 0m. } & .26 & .11 & .24 & .08 & .22 & .01 \\ \text { CPT Simple Com. } & .16 & .05 & .15 & -.03 & .04 & -.06 \\ \text { CPT Compl. Om. } & .26 & .30^{+} & .32^{+} & .45^{*} & -.06 & .27 \\ \text { CPT Compl. Com. } & .08 & -.03 & -.20 & -.15 & -.06 & -.02\end{array}$

Table 5a. Spearman Correlation Coefficienta.

\section{Diaqnostic Group: schizophrenia.}

CNV Cz Early Comp. -

CNV Cz Late Comp. . $82^{* *} \quad-$

CNV Fz Early Comp. . $.86^{* *} \quad .66^{* *} \quad-$

CNV Fz Late Comp. $\quad .76^{* *} \quad .88^{* *} \quad .77^{* *}$

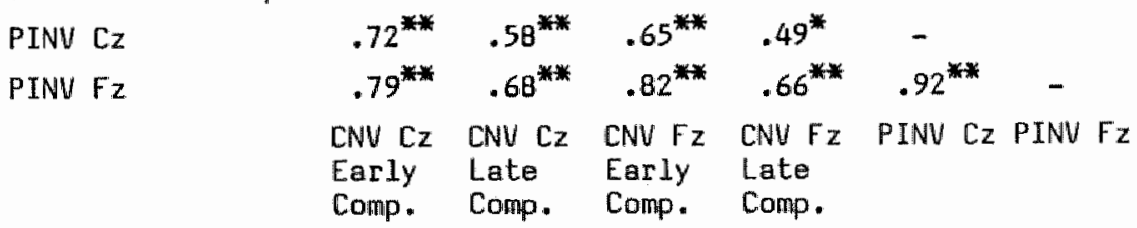


Table 5b. Spearman Correlation Coefficients.

\section{Diagnostic Group: non-schizophrenic psychosis.}

CNV Cz Early Comp. -

CNV Cz Late Comp. $.79^{* * *}-$

CNV Fz Early Comp. $.67^{* *} .61^{* *}-$

CNV Fz Late Comp. $\quad .34^{+} \quad .60^{* *} \quad .66^{* *}=$

PINU $\mathrm{Cz}$

$.60^{* *} \quad .73^{* *} \quad .57^{* *} \quad .47^{*}$

PINV FZ

$.41^{*} \quad .48^{*} \quad .65^{* *} \quad .53^{* *} \quad .69^{* * *} \quad-$

CNV $\mathrm{Cz} \quad \mathrm{CNU} \mathrm{CZ} \quad \mathrm{CNV} \mathrm{Fz}$ CNU $\mathrm{FZ}$ PINV $\mathrm{CZ}$ PINU $\mathrm{Fz}$ Early Late Early Late

Comp. Comp. Comp. Comp.

Table 5c. Spearman Correlation Coefficients.

Diaqnostic Group: schizotypal personality disorder.

CNN Cz Early Comp. -

CNV Cz Late Comp. $\quad .20$

CNV Fz Early Comp. .61** .00

CNV Fz Late Comp. $\begin{array}{lllll}-.10 & .80^{* *} & .01 & -\end{array}$

PINW Cz

$\begin{array}{llll}.12 & .32^{+} & -.11 & .23\end{array}$

PINV Fz

$\begin{array}{lllll}-.01 & .05 & .31^{+} & .28 & .51^{* *}\end{array}$

CNV $\mathrm{CZ} \quad \mathrm{CNN} \mathrm{CZ} \quad \mathrm{CNV} \mathrm{FZ}_{Z}$ CNV $\mathrm{FZ}$ PINU $\mathrm{CZ}$ PINV $\mathrm{Fz}$

Early Late Early Late

Comp. Comp. Comp. Comp. 
Iable 5d. Spearman Correlation Coefficients. Diaqnostic Group: neurosis.

CNV Cz Early Comp. -

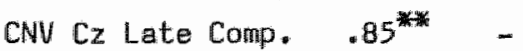

CNV Fz Early Comp. . $80^{* *} \quad .59^{* * *}-$

CNU Fz Late Comp. $\quad .56^{* *} \quad .80^{* *} \quad .43^{*}$

$\begin{array}{llllll}\text { PINV Cz } & .51^{*} & .38^{+} & .30 & .29 & - \\ \text { PINV Fz } & .47^{*} & .25 & .49^{*} & .27 & .74^{*}\end{array}$

CNV $\mathrm{CZ}$ CNV $\mathrm{CZ}$ CNV $\mathrm{Fz}$ CNV $\mathrm{FZ}$ PINV $\mathrm{CZ}$ PINV $\mathrm{FZ}$ Early Late Early Late

Comp. Comp. Comp. Comp.

Iable 5e. Spearman Correlation Coefficients. Diaqnostic Group: normal.

CNU Cz Early Comp. -

CNU Cz Late Comp. $.83^{* *}$ -

CNV Fz Early Comp. $.82^{* *} \quad .66^{* *} \quad-$

CNV Fz Late Comp. $\quad .65^{* *} \quad .82^{* *} \quad .76^{* *}$

PINV $\mathrm{CZ}$

$.60^{* *} \quad .68^{* * *} \quad .33^{*} \quad .44^{*} \quad-$

PINV FZ

$.59^{* *} \quad .70^{* *} \quad .51^{*} \quad .66^{* *} \quad .73^{* *} \quad-$

CNV $\mathrm{CZ} \quad \mathrm{CNV} \mathrm{CZ}$ CNV $\mathrm{Fz}$ CNV $\mathrm{FZ}$ PINV $\mathrm{CZ}$ PINV $\mathrm{FZ}$ Early Late Early Late

Comp. Comp. Comp. Comp. 
Table 6a. Principal Factor Analysis of Attentional Variables. Varimax Rotated Factor Matrix.

Factor I Factor II Factor III Factor IV

$\begin{array}{lrrrr}\text { SPEM Total } & .13 & .18 & .71 & .03 \\ \text { SPEM Best Cycle } & .10 & .22 & .97 & -.05 \\ \text { RT Mean } & .33 & .53 & .25 & -.07 \\ \text { RT Red.Def. } & -.11 & .07 & .13 & .08 \\ \text { CPT Simple Om. } & .14 & .74 & .19 & -.24 \\ \text { CPT Simple Com. } & -.11 & .68 & .05 & .14 \\ \text { CPT Compl. Om. } & .23 & .83 & .22 & -.21 \\ \text { CPT Compl. Com. } & -.13 & .60 & .09 & .10 \\ \text { CNV CZ E.C. } & .78 & .08 & .16 & .26 \\ \text { CNV CZ L.C. } & .89 & .08 & .15 & .12 \\ \text { CNV Fz E.C. } & .73 & .03 & .00 & .35 \\ \text { CNV FZ L.C. } & .71 & .02 & -.04 & .14 \\ \text { PINV Cz } & .38 & -.07 & .01 & .76 \\ \text { PINV Fz } & .39 & -.05 & .03 & .82\end{array}$

\begin{tabular}{|c|c|}
\hline en value & $\begin{array}{l}\text { Percentage of } \\
\text { variance }\end{array}$ \\
\hline
\end{tabular}

$\begin{array}{lrrr}\text { Factor I } & 4.25 & 30.4 & 30.4 \\ \text { Factor II } & 3.23 & 23.0 & 53.4 \\ \text { Factor III } & 1.33 & 9.5 & 62.9 \\ \text { Factor IV } & 1.24 & 8.9 & 71.8\end{array}$


Table 10a* Spearman Correlation Coefficients. Diagnostic Group: schizophrenia.

$\begin{array}{lccccccc}\text { Aff. Flatt. } & - & & & & & \\ \text { Soc. Isol. } & .38^{*} & - & & & & \\ \text { Prem. Asoc. } & .37^{+} & .33^{+} & - & & & & \\ \text {Psychoticism } & .08 & .26 & .07 & - & & & \\ \text { Extraversion } & -.32^{+} & -.21 & -.04 & -.13 & - & & \\ \text { Neuroticism } & .13 & .24 & .04 & .39^{*} & -.11 & - & \\ \text { Lie } & -.03 & .11 & -.12 & .24 & .16 & -.16 & - \\ & \text { Aff. } & \text { Soc. } & \text { Prem. } & \text { Psych. Extrav. Neur. Lie } \\ & \text { Flatt. } & \text { Isal. } & \text { Asac. } & & & & \end{array}$

Table 10b. Spearman Correlation Coefficients.

Diagnostic Group: non-schizophrenic psychosis.

Aff. Flatt. -

Soc. Isol. .64* -

Prem. Asoc. $.58^{* *} \quad .51^{*} \quad-$

$\begin{array}{llllll}\text { Psychoticism } & .14 & .29 & .40^{*} & -\end{array}$

Extraversion $-.37^{+} \quad-.37^{+} \quad-.22 \quad-.09 \quad-$

$\begin{array}{llllll}\text { Neuroticism } & .63^{* *} & .32^{+} & .51^{*} & .50^{*} & -.23\end{array}$

Li.e

$\begin{array}{lllllll}-.23 & -.44^{*} & -.23 & -.50^{*} & .02 & -.31^{+}\end{array}$

Aff. Soc. Prem. Psych. Extrav. Neur. Lie Flatt. Isol. Asoc. 
Table 10c. Spearman Correlation Coefficients.

Diaqnostic Group: schizotypal personality disorder.

Aff. Flatt. -

Soc. Isol. $.32^{+}-$

Prem. Asoc. $.32^{+} \quad .10 \quad-$

$\begin{array}{llllllll}\text { Psychoticism } & .05 & .09 & .36^{+} & -\end{array}$

Extraversion $-.34^{+} \quad-.20 \quad-.12 \quad .37^{+} \quad-$

$\begin{array}{llllllll}\text { Neuroticism } & -.27 & .11 & -.07 & -.16 & -.32^{+} & -\end{array}$

Lie

$\begin{array}{llllll}-.07 & -.19 & -.17 & -.28 & .04 & -.07\end{array}$

Aff. Soc. Prem. Psych. Extrav. Neur. Lie Flatt. Isol. Asoc.

Table 10d. Spearman Correlation Coefficients. Diagnostic Group: neurosis.

$\begin{array}{lccccccc}\text { Aff. Flatt. } & - & & & & & \\ \text { Soc. Isol. } & .36^{+} & - & & & & & \\ \text {Prem. Asoc. } & .23 & .45^{*} & - & & & & \\ \text { Psychoticism } & .05 & -.26 & -.19 & - & & & \\ \text { Extraversion } & -.14 & -.21 & -.45^{*} & .39^{*} & - & & \\ \text { Neuroticism } & .01 & -.31^{+} & -.01 & -.09 & -.20 & - & \\ \text { Lie } & -.01 & .15 & .24 & -.18 & -.13 & -.64^{* * *} & - \\ & \text { Aff. } & \text { Soc. } & \text { Prem. } & \text { Psych. Extrav. Neur. Lie } \\ & \text { Soc. } & \text { Isol. Asoc. } & & & & \end{array}$


Iable 10e. Spearman Correlation Coefficients.

Diagnostic Group: normal.

Aff. Flatt. -

Soc. Isol. $.69^{* *}-$

Prem. Asoc. .38* $.39^{*} \quad-$

$\begin{array}{llllll}\text { Psychoticism } & .24 & .00 & -.31^{*} & -\end{array}$

Extraversion - $-.38^{*} \quad-.26 \quad-.43 * \quad .18 \quad-$

$\begin{array}{lllllll}\text { Neuraticism } & .20 & .42 * & .44 * & -.10 & -.18 & -\end{array}$

Lie

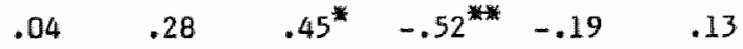

Aff. Soc. Prem. Psych. Extrav. Neurot. Lie Soc. Isol. Asoc.

Table 13a. Principal Factor. Analysis of Diannostic Variables. Varimex Rotated Factor Matrix.

\author{
Factor I Factor II
}

$\begin{array}{lrr}\text { Affective Flattening } & .83 & -.03 \\ \text { Social Isolation } & .76 & -.02 \\ \text { Premorbid Asociality } & .67 & .03 \\ \text { Psychoticism } & .16 & .75 \\ \text { Extraversion } & -.58 & .32 \\ \text { Neuroticism } & .51 & -.06 \\ \text { Lie } & .13 & -.31\end{array}$

Eigen value

Percentage of variance

2.85

1.28
40.7

18.2
Cumulative percentage

$\begin{array}{llll}\text { Factor I } & 2.85 & 40.7 & 40.7 \\ \text { Factor II } & 1.28 & 18.2 & 59.0\end{array}$


Iable 29a. Spearman Correlation Coefficients.

\section{Diagnostic Group: schizophrenia.}

Aff. Soc. Prem. $\mathbb{P}$ E $N$ L

Flatt. Isol. Asoc.

$\begin{array}{lrrrrrrr}\text { SPEM Total } & .02 & .13 & .01 & .46^{*} & -.01 & .48^{*} & .05 \\ \text { SPEM Best Cycle } & -.13 & .17 & -.13 & .33^{+} & -.12 & .39^{*} & .05 \\ \text { RT Mean } & .15 & .16 & .16 & .24 & .16 & .04 & .21 \\ \text { RT Red.Def. } & -.12 & .14 & .10 & -.21 & .53^{* *} & -.59^{* *} & .26 \\ \text { CPT Simple Om. } & -.07 & .00 & .16 & .50^{*} & .02 & .10 & .30^{+} \\ \text {CPT Simple Com. } & .12 & -.26 & .05 & .28 & .01 & -.10 & -.01 \\ \text { CPT Compl. Om. } & .11 & .11 & .38^{*} & .25 & -.23 & .02 & .26 \\ \text { CPT Compl. Com. } & -.01 & -.09 & -.17 & .07 & .08 & -.02 & -.07 \\ \text { CNV Cz E.C. } & .11 & .33^{+} & .08 & .11 & -.37^{+} & .10 & .12 \\ \text { CNV Cz L.C. } & .13 & .50^{* *} & -.05 & .26 & -.51^{* *} & .17 & -.03 \\ \text { CNV Fz E.C. } & .40^{*} & .28 & .27 & .09 & -.47^{*} & .09 & .10 \\ \text { CNV Fz L.C. } & .25 & .36^{+} & .01 & .20 & -.54^{* *} & .20 & .00 \\ \text { PINU Cz } & .14 & .27 & .16 & .14 & -.06 & .05 & -.06 \\ \text { PINU Fz } & .29 & .31^{+} & .16 & .14 & -.011^{+} & .01 & -.05\end{array}$


Table 29b. Spearman Correlation Coefficients.

Diagnastic Group: non-schizophrenic psychasis.

Aff. Soc. Pren. P $\quad$ E $N$

Flatt. Isol. Asoc.

$\begin{array}{lrrrrrrl}\text { SPEM Total } & -.42^{*} & -.25 & .02 & .34^{+} & .13 & .04 & .12 \\ \text { SPEM Best Cycle }-.22 & -.23 & .06 & .07 & .01 & -.04 & .38^{*} \\ \text { RT Mean } & .24 & .14 & .02 & .21 & -.31^{*} & .11 & .13 \\ \text { RT Red.Def. } & -.10 & .09 & -.09 & -.08 & .03 & -.23 & .01 \\ \text { CPT Simple Om. } & .33^{+} & -.09 & .12 & .02 & -.14 & .24 & -.05 \\ \text { CPT Simple Com. } & -.01 & -.07^{*} & .00 & .03 & -.04 & .04 & .29 \\ \text { CPT Compl. Om. } & .29 & .02 & .29 & .07 & -.34^{*} & .32^{*} & .07 \\ \text { CPT Compl. Com. } & -.06 & .03 & .09 & .19 & -.06 & .11 & -.12 \\ \text { CNV Cz E.C. } & .10 & -.25 & -.01 & -.32^{+} & .23 & .04 & .47^{*} \\ \text { CNV Cz L.C. } & .18 & -.10 & -.07 & -.16 & .40^{*} & .25 & .23 \\ \text { CNV Fz E.C. } & -.02 & -.06 & .02 & -.38^{*} & .23 & -.11 & .46^{*} \\ \text { CNV Fz L.C. } & .30 & .23 & .19 & -.01 & .14 & .26 & .10 \\ \text { PINV Cz } & -.02 & -.34^{*} & -.22 & -.17 & .41^{*} & .10 & .44^{*} \\ \text { PINV Fz } & .10 & -.09 & -.09 & -.28 & .22 & .06 & .34^{+}\end{array}$


Table 29c. Spearman Correlation Coefficients.

Diagnostic Group: schizotypal personality disorder.

Aff. Soc. Prem. P $E$ N

Flatt. Isal. Asac.

$\begin{array}{lrrrrrrr}\text { SPEM Total } & -.35^{+} & -.08 & -.23 & .03 & .17 & -.15 & .09 \\ \text { SPEM Best Cycle } & -.27 & .01 & .01 & .00 & .36^{+} & -.31^{+} & -.03 \\ \text { RT Mean } & .15 & .21 & -.17 & .20 & .43^{*} & -.23 & .32^{*} \\ \text { RT Red.Def. } & .00 & -.01 & .07 & -.09 & .31^{+} & -.35^{+} & .03 \\ \text { CPT Simple 0m. } & .21 & .26 & -.13 & .25 & .44^{*} & -.31^{+} & -.37^{+} \\ \text {CPT Simple Com. } & .16 & .02 & .01 & -.03 & -.18 & .18 & -.27 \\ \text { CPT Compl. Om. } & .29 & .12 & -.22 & .13 & .33^{+} & -.28 & -.22 \\ \text { CPT Compl. Com. } & .39^{*} & .16 & -.17 & -.08 & -.12 & .01 & -.09 \\ \text { CNV Cz E.C. } & -.27 & -.07 & .09 & .17 & .44^{*} & .25 & -.45^{*} \\ \text { CNV Cz L.C. } & -.30 & -.45^{*} & .06 & .42^{*} & .27 & .16 & .26 \\ \text { CNV Fz E.C. } & -.16 & .25 & .07 & .00 & .22 & .24 & -.45^{*} \\ \text { CNV Fz L.C. } & -.14 & -.38^{+} & -.20 & .20 & .18 & .11 & .37^{+} \\ \text {PINV Cz } & -.35^{*} & -.30 & .01 & .10 & .11 & .18 & .10 \\ \text { PINV Fz } & -.20 & .09 & -.15 & .07 & .06 & .18 & -.17\end{array}$


Iable 29d. Spearman Correlation Coefficients.

Diagnostic Group: neurasis.

Aff. Soc. Prem. P $\quad$ E $N$ N

Flatt. Isol. Asoc.

$\begin{array}{lccccccr}\text { SPEM Total } & .13 & .26 & .29 & -.07 & -.16 & -.44^{*} & .17 \\ \text { SPEM Best Cycle } & .06 & .15 & .31^{+} & -.22 & -.11 & -.19 & .07 \\ \text { RT Mean } & .35^{+} & -.09 & .30^{+} & .03 & -.35^{+} & .14 & .07 \\ \text { RT Red.Def. } & .03 & -.16 & .06 & .09 & -.07 & .23 & -.08 \\ \text { CPT Simple Om. } & .15 & .04 & .40^{*} & -.10 & -.15 & .14 & -.05 \\ \text { CPT Simple Com. } & -.01 & -.22 & -.50^{*} & .10 & .33^{+} & -.30^{+} & .02 \\ \text { CPT Compl. Om. } & .06 & .30 & .42^{*} & -.19 & -.25 & -.10 & .09 \\ \text { CPT Compl. Com. } & .06 & .23 & .02 & -.04 & .09 & -.02 & -.09 \\ \text { CNV Cz E.C. } & .24 & -.24 & .41^{*} & .26 & -.10 & .13 & .03 \\ \text { CNV Cz L.C. } & .30^{+} & -.13 & .49^{*} & .22 & -.09 & .22 & .12 \\ \text { CNV Fz E.C. } & -.16 & -.34^{+} & .21 & .45^{*} & .12 & -.01 & .07 \\ \text { CNV Fz L.C. } & .24 & -.18 & .23 & .12 & .15 & .34^{+} & .01 \\ \text { PINV CZ } & .11 & -.50^{*} & -.10 & .26 & .25 & .01 & .03 \\ \text { PINV FZ } & -.27 & -.37^{+} & -.09 & .25 & .28 & -.01 & -.11\end{array}$


Table 29e. Spearman Correlation Coefficients.

\section{Diagnostic Graup: normal.}

Aff. Soc. Prem. P $\quad$ E $N$ N

Flatt. Isol. Asoc.

$\begin{array}{lccccccc}\text { SPEM Total } & -.26 & -.23 & .04 & .03 & .14 & -.46^{*} & .15 \\ \text { SPEM Best Cycle } & -.14 & -.17 & .00 & -.14 & .21 & -.36^{*} & .26 \\ \text { RT Mean } & -.16 & -.01 & .17 & -.26 & -.44^{*} & -.11 & .44^{*} \\ \text { RT Red.Def. } & .14 & -.14 & .07 & .33^{*} & -.27 & .25 & -.20 \\ \text { CPT Simple Om. } & .25 & .23 & -.05 & .10 & -.05 & .23 & .17 \\ \text { CPT Simple Com. } & .19 & .05 & .06 & .11 & .15 & -.14 & -.01 \\ \text { CPT Compl. Dm. } & -.27 & -.21 & -.22 & .41^{*} & .20 & .02 & -.19 \\ \text { CPT Compl. Com. } & -.22 & -.32^{+} & .20 & -.24 & .10 & .08 & .24 \\ \text { CNV Cz E.C. } & .30 & .23 & .18 & .09 & -.31^{+} & -.05 & .10 \\ \text { CNV CZ L.C. } & .18 & .29 & .04 & -.02 & -.27 & -.20 & .18 \\ \text { CNV Fz E.C. } & .34^{+} & .17 & .04 & .37^{+} & -.36^{+} & -.14 & -.07 \\ \text { CNV Fz L.C. } & .10 & .09 & -.12 & .11 & -.28 & -.20 & -.04 \\ \text { PINV Cz } & .30 & .32^{+} & .11 & -.22 & -.41^{*} & -.15 & .24 \\ \text { PINV Fz } & -.10 & -.06 & -.17 & .02 & -.07 & -.043^{*} & .01\end{array}$




\section{REFERENCES}

ABRAHAM, P." MCCALLUM, W.C., DOCHERTY, T., FOX, A. and NEWTON, P. (1974) The CNV in schizophrenia. Electroenceph.clin. Neurophysiol. 36, $217-218$.

ABRAHAM, P., MCCALLUM, W.C. and GOURLAY, J. (1976) The CNU and its relation to specific psychiatric syndromes. In: W.C. MeCallum and J.R. Knott (Eds.) The responsive brain. Bristol, John Wright and Sons.

ABRAHAM, P. and MCCALLUM, W.C. (1977) A permanent change in the EEG (CNV) of schizophrenics. Electroenceph.clin.Neurophysiol. 43, 533.

ABRAMS, R. and TAYLOR, M.A. (1978) A rating scale for emotional bliunting. Am. J.Psychiat. $135,226-229$.

ACKER, W. and TOONE, B. (1978) Attention, eye tracking and schizophrenia. Br.J.soc.clin.Psychol. 17, 173-181.

ALPERN, M. (1972) Eye movements. In: D. Jameson and L.M. Hurvich (Eds.) Handbook of sensory physiology, Vol. VII/4. Visual psychophysics. Berlin, Springer Verlag.

AMERICAN PSYCHIATRIC ASSOCIATION (1968) Diagnostic and statistical manual of mental disorders, 2nd edition. Washington, A.P.A.

AMERICAN PSYCHIATRIC ASSOCIATION (1980) Diagnostic and statistical manual of mental disorders, $3 \mathrm{rd}$ edition. Washington, A.P.A.

ANDREASEN, N.C. (1979) Affective flattening and the criteria for schizophrenia. Am.J.Psychiat. 136, 944-947.

ASARNDW, R.F. and MacCRIMMON, D.J. (1978) Residual performance deficit in clinically remitted schizophrenia: a marker of schizophrenia? 3.Abnorm.Psychol. 87, 597-608.

ASARNOW, R.F., STEFFY, R.A., MaCCRIMMON, D.J, and CLEGHORN, J.M. (1977) An attentional assessment of foster children at risk for schizophrenia. J.Abnorm. Psychol. 86, 267-275. 
ASARUNOW, R.F., STEFFY, R.A. MaCCRIMMON, D.J. and CLEGHORN, J.M. (1978) An attentional assessment of foster children at risk for schizophrenia. In: L.C. Wynne, R.L. Cromwell and S. Matthijsse (Eds.) The nature of schizophrenia. New approaches to research and treatment. New York, Wiley.

ASHTON, H., MILLMAN, J.E., TELFDRD, R. and THOMPSON, J.W. (1974) The effect of caffeine, nitrazepam and cigarette smoking on the contingent negative variation in man. Electroenceph.clin. Neurophysiol. 37, 59-71.

ASTRACHAN, B.M. HARRDW, M., ADLER, D., BRAUER, L., SCHWARTZ, A., SCHWARTZ, C. and TUCKER, G. (1972) A checklist for the diagnosis of schizophrenia. Brit.J.Psychiat. 121, 529-539.

ASTRUP, $\mathbb{C}$. and NORE $K$, K. (1966) Functional psychoses: diagnostic and prognostic models. Springfield, Illinois, Charles C. Thomas.

BARCHAS, J.D., ELLIOT, G.R. and BERGER, P.A. (1978) Biogenic amine hypotheses of schizophrenia. In: L.C. Wymne, R.L. Cromurel1 and 5. Matthijase (Eds.) The nature of schizophrenia. New approaches to research and treatment. New York, Wiley.

BATESON, G., JACKSON, D.D., HALEY, J. and WEAKLAND, J.H. (1956) Towards a theory of schizophrenia. Behav.Sc. 1, 251-264.

BATESON, G. (1972) Form, substance and difference. In: Steps to an ecology of mind. Chandler.

BECKNER, M. (1959) The biological way of thought. New York, Columbla University Press.

BELLISSIMO, A. and STEFFY, R.A. (1972) Redundancy-associated deficit in schizophrenic reaction time performance. J.Abnorm.Psychol. 80, 299-307.

BELLISSIMO, A. and STEFFY, R.A. (1975) Contextual influences on crossover in the reaction time performance of schizophrenics. J.Abnorm. Psychol. 84, 210-220.

BERLYNE, D.E. (1970) Attention as a problem in behavior therapy. In: D. 1. Mogtofsky (Ed.) Attention: Contemporary theory and analysis. New York, Appleton-Century-Crofts.

BLAND, R.C. and ORN, H. (1979) Schizophrenia: diagnostic criteria and outcome. Brit.J. Psychiat. 134, 34-38.

BLAND, R.C. and ORN, H. (1980) Schizophrenia: Schneider"s first rank symptoms and outcome. Brit.J.Psychiat. 137, 63-68.

BLASHFIELD, R.K. (1976) Mixture model tests of cluster analysis: Accuracy of four agglomerative hierarchical methods. Psychol.Bull. 83, $377-388$. 
BLASHFIELD, R.K. and ALDENDERFER, M.S. (1979) The literature on cluster analysis. Multivariate Behaw. Res. 13, 271-295.

BLEULER, E. (1911) Dementia Praecox oder Gruppe der Schizophrenien. Leipzig, Franz Deuticke.

BLEULER, M. (1972) Die schizophrenen Geistesstörungen im Lichte lang jâhriger Kranken- und Familiengeschichten. Stuttgart, Thieme. English translation (1978): The schizophrenic disorders: long-term patient and family studies. Newhaven, Yale University Press.

BLOCK, J. (1977) The Eysencks and psychoticism. J.Abnorn.Psycho1. 66, $653-654$.

BLDCK, "I. (1978) Claridge and Birchal, different forms of the P scale, and dependable moderators. J.Abnorm. Psychol. 87, 669-672.

BORING, E.C. (1929) A history of experimental psychology. Appleton-Century.

BOTWINICK, J., BRINLEY, J.F. and ROBBIN, J.5. (1959) Maintaining set in relation to motivation and age. Am.J.Psycho1. 72, 585-58B.

BOTWINICK, J. and THDMPSON, L.W. (1966) Premotor and motor components of reaction time. J.Exp.Psychol. 71, 9-15.

BDUERS, M.B. (1980) Biochemical processes in schizophrenia: An update. Schizophrenia Bu11. 6, 393-403.

BRAFF, D.L. (1981) Impaired speed of information processing in nonnedicated schizotypal patients. Schizophrenia Bull. 7, 499-508.

BREZINOWA, V. and KENDELL, R.E. (1977) Smooth pursuit eye movements of schizophrenics and normal people under stress. Brit.J.Psychilat. 130, 59-63.

BROADBENT, D.E. (1958) Perception and communication. London, Pergamon Press.

BROADBENT, D.E. (1970) Stimulus set and response set. Two kinde of sellective attention. In: D.I. Mostofsky (Ed.) Attention: contemporary theory and analysis. New York, Appleton-Century-Crofts.

BROCKINGTON, I.F., KENDELL, R.E. and LEFF, J.P. (1978) Definitions of schizophrenia: concordance and prediction of outcome. Psychol.Med. B, $387-398$.

BROEN, W.E. (1966) Response disorganization and breadth of observation in schizophrenia. Psychol.Rev. 73, 579-585.

BROEN, W.E. (1968) Schizophrenia. Research and theory. New York, Academic Press. 
BROEN, W.E. and STORIAS, L.H. (1966) Layful disorganization: The process underlying a schizophrenic syndrome. Psychol.Rev. 73, 265-279.

BROEN, W.E. and STORHS, L.H. (1967) A theory of response interference in schizophrenia. In: B.A. Maher (Ed.) Progress in experimental personality research, Vol. 4. New York, Academic Press.

BROWN, G.W., BIRLEY, J.L.T. and WING, J.K. (1972) Influence of family life on the course of schizophrenic disorders: a replication. Brit.J.Psychiat. 121, 241-258.

BUCHSBAUM, M.S., INGVAR, D.H., KESSLER, R., WATERS, R.N., CAPPELLETTI, J., VAN KAMMEN, D.P., KING, A.C., JOHNSON, J.L., MANNING, R.G., FLYNN, R.W., MANN, L.S., BUNNEY, W.E. and SOKDLOF, L. (1982) Cerebral glucography with positron tomography. Use in normal subjects and in patients with schizophrenia. Arch. Cen.Psychiatry 39, 251-259.

BUCHSBAUM, M.S., MURPHY, D.L., COURSEY, R.D., LAKE, C.R. and ZEIGLER, M.G. (197B) Platelet monoamine oxidase, plasma dopamine-betahydroxyllase and attention in a 'biochemical high-risk' sample. In: L.C. Wynne, R.L. Cromell and 5. Matthijase (Eds.) The nature of schizophrenia. New approaches to research and treatment. New York, villey.

BUCKNER, D.N. and MCGRATH, J.J. (Eds.) (1963) Vigilance: a symposium. New York, McGraw-Hill.

CALLAWAY, E. (1978) The psychophysiology of schizophrenia. In: W.E. Fann, I. Karacan, A.D. Pokorny and R.L. Willians (Eds.) Phenomenology and treatment of schizophrenia. New York, Spectrum Publications.

CARLSSON, A. (1978) Does dopamine have a role in schizophrenia? Biol. Psychiat. 13, 3-21.

CARPENTER, W.T., SACKS, M.H., STRAUSS, J.S., BARTKO, J.J. and RAYMER, J. (1976) Evaluating signs and symptoms: comparison of structured interview and clinical approaches. Brit.J.Psychiat. 128, $397-403$.

CARPENTER, W.T., STRAUSS, J.S. and BARTKO, J.J. (1973) Flexible system for the diagnosis of schizophrenia: report from the WHO International Pilot Study of Schizophrenia. Science 182, 1275-1278.

CEGALIS, J.A. and SWEENEY, J.A. (1979) Eye movements in schizophrenia: a quantitative analysis. Biol.Psychiat. 14, 13-26.

CHAPMAN, L.J. and CHAPMAN, J.P. (1973) Disordered thought in schizophrenia. New York, Appleton-Century-Crofts.

CHAPMAN, L.J. and CHAPMAN, J.P. (1980) Scalles for rating psychotic and psychotic-like experiences as continua. Schizophrenia Bull. 6, 476-489. 
CHAPMAN, L.J., CHAPMAN, J.P. and RALLIN, M.L. (1976) Scales for physical and social anhedonia. J.Abnorm.Psychol. 85, 374-382.

CHAPMAN, L.J., CHAPMAN, J.P. and RAULIN, M.L. (1978) Body-image aberration in schizophrenia. J.Abnorm. Psychol. 87, 399-407.

CHAPMAN, L.J., EDELL, W.S. and CHAPMAN, J.P. (1980) Physical anthedoni.a, perceptual aberration, and psychosis proneness. Schizophrenia Bull. 6, 639-653.

CHOUINARD, G., ANNABLE, L. and DONGIER, M. (1977) Differences in psychopathology of schizophrenic patients with normal and abnormal post imperative negative variation (PINV) Compr.Psychiatry 18, 83-87.

CHOUINARD, G., ANNABLE, L., DUBRONSKY, B. and DONGIER, M. (1975) Pastimperative negative variation (PINV) in ambulatory schizophrenic patients. Compr.Psychiatry 16, 457-460.

CIOMPI, L. (1980) Catamnestic long-term study on the course of life and aging of schizophrenics. Schizophrenia Bull. 6, 606-618.

CIOMPI, L. and MULLER, C.H. (1976) Lebensweg und Alter der Schizophrenen. Eine katamnestische Langzeitstudie bis ins Senium. Berlin, Springer.

CLARIDGE, G.S. (1960) The excitation-inhibition balance in neurotics. In: H.J. Eysenck (Ed.) Experiments in personality. London, Routledge and Kegan Paul.

CLARIDGE, G.S. (1967) Personality and arousal. A psychophysiological study of psychiatric disorder. Oxford, Pergamon.

CLARIOGE, G. (1972) The schizophrenias as nervous types. Brit.J. Psychiat. 121, 1-17.

CLARIDGE, G. and BIRCHAL, P. (1978) Comments. Bishop, Eysenck, Block and psychoticism. J.Abnorm. Psychol. 87, 664-668.

CLARIDGE, G.5. and CHAPPA, H.J. (1973) Psychoticism: A study of Its biological basis in normal subjects. Br.J.soc.clin.Psychol. 12, 175-187.

CLARIDGE, G.S. and HUME, W.I. (1966) Comparioon of effects of dexamphetamine and $L S D-25$ on perceptual and autonomic function. Percept.mot.Skills 23, 456-458.

COHLER, B.J., GRUNEBAUM, H.H., WEISS, J.L., GAMER, E. and GALLANT, D.H. (1977) Disturbance of attention among schizophrenic, depressed and well mothers and their young children. J.Child.Psychol. Paychiat. $18,115-135$. 
COOPER, J.E. KENDELL, R.E.* GURLAND, B.J., SHARPE, L. , COPELAND, J.R.M. and SIMON, R. (1972) Psychiatric diagnosis in Mev York and London. Mauds ley Monograph No. 20. London, Oxford University Presi.

CORNIMG, W.C. and STEFFY, R.A. (1979) Taximetric strategies applied to peychiatric claseification. Schizophrenia Bul1. 5, 294-305.

CQUCH, F.H. and FOX, J.C. (1934) Photographic study of ocular movements in mental disease. Arch. Neurol. Psychiatry 34,556-578.

CROMWELL, R.L. (1975) Assegsment of schizophrenia. In: M.R. Rozenzweig and L.W. Porter (Eda.) Annual review of psychology. Nol. 26.

CROMWELL, R.L. (1978) Conclusion. J.Psychiat.Res. 14,327-331. Also In: 5. Matthijsse, B.J. Spring and J. Sugarman (Eds.) Attention and information processing in schizophrenia. Oxford, Pergemon, 1979.

CROMWELL, R.L., DeAMICIS, L., HAYES, T. and BRIGGS, D. (1979) Reaction time crossover, vulnerability index: mean reaction time, a symptom severity index. Psychopharmacol. Bu11. 15, 24-25.

CROMWELL, R.L., ROSENTHAL, D., SHAKOW, D. and ZAHN, T.P. (1961) Reaction time, Jocus of control, choice behavior, and descriptions of parental behavior in schizophrenic and normal subjects. J.Pers. 29, 363-379.

DAVIES, D.R. and TUNE, G.5. (1970) Human vigilance performance. London, Staples Press.

DeAMICIS, L.A. and CROMWELL, R.L. (1979) Reaction time crossover in procesa schizophrenic patients, their relatives, and control subjects. J.Nerv. Ment.Dis. 167, 593-600.

DECKER, H.S. (1978) The historical evolution of dementia praecox. In: W.E. Fann, I. Karacan, A.D. Pokorny and R.L. Williams (Eds.)

Phenomenology and treatment of schizophrenia. New York, Spectrum.

DEECKE, L. and KORNHUBER, H.H. (1977) Cerebral potentials and the initiation of voluntary movement. In: J.E. Desmedt (Ed.) Attention, voluntary contraction and event-related cerebral potentials. Prog*clin. Neurophysiol., Vol. 1. Basel, Karger.

DELAUNOY, J., TIMSIT-BERTHIER, M., ROUSSEAU, J.C. and GERONO, A. (1975-a) Experimental modification of the terminal phase of the contingent negative variation. Electroenceph.clin. Neurophysiol. 38, 551.

DELALNOY, J., TIMSIT-BERTHIER, M. , ROUSSEAU, J.C. et GEROND, A. (1975-b) Modification experimentale de la phase terminale de la VCN. Rev.EG Neurophysiol. 5, 10-14.

DELL, P.F. (1980) Researching the family theories of schizophrenia: Am exerciae in epistemological confusion. Family Process 19, 321-335. 
DEPUE, R.A. (1974) The specificity of response interference to schizophrenia. J.Abnorm. Psychol. 83, 529-532.

DESMEDT, J.E., DEBECKER, J. and ROBERTSON, D. (1979) Sorlal perceptual processing and the neural basis of changes in event-related potentials components and slow potential shifts. In* J.E. Desmedt (Ed.) Cognitive components in cerebral event-related potentials and selective attention. Prog.clin. Neurophysiol. Vol. 6. Basel, Karger.

DIEFENDORF, A.R. and DODGE, R. (1908) An experimental study of the ocular reactions of the insane from photographic records. Brain 31 , $451-489$.

DONGIER, M. (1973) Clinical applications of the CNV. A review. In: W.C. MACCallum and J.R. Knott (Eds.) Event-related slow potentials of the brain: their relations to behavior. Electroenceplh.clin. Neurophysiol., Suppl. 33 .

DONGIER, M., DUBROUSKY, B. et GARCIA-RILL, E. (1974) Les potentiels cerebraux lents en psychiatrie. Can.Psychiatr.Assoc.J. 19, 177-183.

DONGIER, M., TIMSIT-BERTHIER, M., KONINCKX, N. and DELAUNOY, J. (1973) Compared clinical significance of CNV and other slow potential changes in psychiatry. In: W.C. McCallum and J.R. Knott (Eds.) Event-related slow potentials of the brain: their relations to behavior.

Electroenceph.clin.Neurophysiol., Suppl. 33.

DOWNING, A.R., FRANCIS, A.F. and BROCKINGTON, I.F. (1980) A comparison of information sources in the study of psychotic illness. Brit..]. Psychiat. $137,38-44$.

DUEROUSKY, B. and DONGIER, M. (1976) Evaluation of event-related slow potentials in selected groups of psychiatric patients. In: W.C. McCallum and J.R. Knott (Eds.) The responsive brain. Bristol, John Wright and Sons.

EASTERBROOK, J.A. (1959). The effect of emotion on cue utilization and the organization of behavior. Psychol. Rev. 66, 183-201.

EPSTEIN, S. (1967) Toward an unified theory of anxiety. In: B.A. Maker (Ed.) Progress in experimental personality research, Vol. 4. New York, Academic Preso.

EPSTEIN, S. and COLEMAN, M* (1970) Drive theories of schizophrenia. Psychosom. Med. 32, 113-140.

ERLENMEYER-KIMLING, L. and CORNBLATT, B. (1978) Attentional measures in a study of children at high-risk for schizophrenia. J.Psychiat.Res. 14, 93-98. Also in: 5. Matthijsse, B.J. Spring and J. Sugarman (Eds.) Attention and information processing in schizophrenia. Oxford, Pergamon, 1979.

EVERITT, B. (1974) Cluster analysis. London, Heinenann. 
EYSENCK, H.J. (1977) Comments. Psychosis and psychoticism: a reply to Biahop. J.Abnorm. Paychol. 86, 427-430.

EYSENCK, H.J. and EYSENCK, S.B.G. (1975) Manual of the Eysenck Personality Questionnaire. London, Hodder and Stoughton.

EYSENCK, H.J. and EYSENCK, S.B.G. (1976) Psychoticism as a dimension of personality. London, Hodder and Stoughton.

EYSENCK, H.J. and EYSENCK, S.B.G. (1977) Comments. Block and psychoticism. J.Abnorm. Paycho1. 86, 651-652.

FEIGHNER, J.P., ROBINS, E., GUZE, S.B. , WOODRUFFE, R.A., WINOKUR, G. and MUNOZ, R. (1972) Diagnastic criteria for use in paychiatric research. Arch. Gen.Psychiatry 26,57-63.

FENTON, G.W., FENWICK, P.B.C., FERGUSON, W. and LAM, C.T. (1978) The contingent negative variation in antisocial behaviour: a pilot study of broadmoor patients. Brit.J.Psychiat. 132, 368-377.

FENTDN, W.S., MOSHER, L.R. and MATTHEWS, S.M. (1981) Diagnosis of schizophrenia: a critical review of current diagnostic systems. Schizophrenia Bul1. 7, 452-476.

FORREST, F.M. FORREST, I.S. and MASON, A.S. (1961) Review of rapid urine tests for phenothiazine and related drugs. Am.J.Psychiat. 118, 300-307.

FRANZEN, G. and INGUAR, D.H. (1975) Abnormal distribution of cerebral activity in chronic schizophremia. J.Psychiat.Res. 12, 199-214.

FREEDMAN, B.J. (1974) The subjective experience of perceptual and cognitive disturbances in schizaphrenia. A review of autobiographical accounts. Arch. Gen.Psychiatry 30, 333-340.

FREEDMAN, B. and CHAPMAN, L.J. (1973) Early subjective experience in schizophrenic episodes. J.Abnorm. Psychol. 82, 46-54.

FUKUI, Y., NAKAMURA, M., KADOBAYASHI, I. and KATOH, N. (1978) The property of contingent negative variation (CNV) in psychiatric patients: schizophrenia and neurosis. Folia Psychiat. Neurol. Jap. 32, $539-552$.

GAILLARD, A.W.K. (1977) The late CNV wave: preparation versus expectancy. Psychophysiol. 14, 563-568.

GARMEZY, N. (1977) The psychology and psychapathalagy of attention. Schizophrenia Bull. 3, 360-369.

GARMEZY, N. (1978) Attentional processes in adult schizophrenia and in children at risk. J.Psychiat.Res. 14, 3-34. Also in: S. Matthijsse, B.J. Spring and J. Sugarman (Eds.) Attention and information processing in schizophrenia. Oxford, Pergamon, 1979. 
GAUTHIER, P. et GOITESMANN, C. (1975) Etude de la variation contingente négative et de 1 'onde post-imperative en présence d"interférences. Electroenceph.clin. Neurophysiol. 40, 143-152.

GILBERSTADT, H. and DUKER, J.A. (1965) Handbook for clinical and actuarial MMPI interpretation. Philadelphia, Saunders.

GITTELMAN-KLEIN, R, and KLEIN, D.F. (1969) Premorbid asocial adjustment and prognosis in schizophrenia. J.Psychiat.Res. 7, 35-53.

GOLDEN, R.R. and MEEHL, P.E. (1979) Detection of the schizoid taxon with MMPI indicators. J.Abnorm. Psychol. 88, 217-233.

GOLDSTEIN, M.J. and RODNICK, E.H. (1975) The family's contribution to the etiology of schizophrenia: Current status. Schizophrenia Bull. $1(14), 48-63$.

GOTTESMAN, I.I. (1978) Schizophrenia and genetics: Where are we? Are you sure? In: L.C. Wymne, R.L. Crome11 and S. Matthijsse (Eds.) The nature of schizophrenia. Ney approaches to research and treatment. New York, Wiley.

GOTTESMAN, I.I. and SHIELDS, J. (1973) Genetic theorizing and schizophrenia. Brit.J.Psychiat. 122, 15-30.

GOTTESMAN, I.I. and SHIELDS, J. (1976) A critical review of recent adoption, twin, and family studies of schizophrenia: behavioral genetics perspectives. Schizophrenia Bull. 2, 360-398.

GRIFFITH, J.H., FRITH, C.D. and EYSENCK, S.B.G. (1980) Psychoticism and thought disorder in psychiatric patients. Brit.J.soc.clin.Psychol. $19,65-71$.

GRINKER, R.R., WERBLE, B. and DRYE, R. (1968) The barderline syndrome: A behavioral study of ego functions. New York, Basic Books.

GROSS, G. and HUBER, G. (1972) Sensorische Störungen bei

Schizophrenien. Arch. Psychiat. Nervenkr. 216, 119-130.

GRUNEBAUM, H., COHLER, B.J., KAUFFMAN, C. and GALLANT, D. (1978)

Children of depressed and schizophrenic mothers. Child Psychiatry Hum. Dev. B, 219-228.

GRUNEBALM, H., WEISS, J.L., GALLANT, D. and COHLER, B.J. (1974) Attention in young children of psychotic mothers. Am.J.Psychiat. 131, $887-891$.

GRUZELIER, J.H. and FLOR-HENRY, P. (Eds.) (1979) Hemisphere asymmetries of function in psychopathology. Amsterdam, Elsevier.

GUNDERSON, J.G. and SINGER, M.T. (1975) Defining borderline patients: An overview. Am.J.Psychiat. 132, 1-10. 
HAMPER, M. and ZUBIN, J. (1968) Evolution, culture, and psychopathology. J.Gen.Psychol. 78, 151-164.

HAASE, H.J. (1963) Möglichkeiten und Grenzen der Psychapharmakotherapie mit Tranquilizern und Neuroleptika. Dtsch.med.Wschr. 88, 505-514.

HABLITZ, J.J. and BORDA, R.P. (1973) The effects of Dalmane (flurazepam hydrochloride) on the contingent negative variation. In: W.C. McCallum and J.R. Knott (Eds.) Event-related sloy potentials of the brain: their relations to behavior. Electroenceph.clin. Neurophysiol., Supp1. 33.

HAIER, R.J., ROSENTHAL, D. and WENDER, P.H. (1978) MMPI assessment of psychopathology in the adopted-way offspring of schizophrenics. Arch.Gen.Psychiatry $35,171-175$.

HASENFUS, N. and MAGARO, P. (1976) Creativity and schizophrenia: an equality of empirical canstructs. Brit.J.Psychiat. 129, 346-349.

HAWK, A.B., CARPENTER, W.T. and STRAUSS, J.S. (1975) Diagnastic criteria and five-years outcone in schizophrenia. A report from the International Pilat Study of Schizophrenia. Arch. Gen.Psychiatry 32, 343-347.

HELD, J.M., CROMWELL, R.L., FRANK, E.J. and FANN, W.E. (1970) Effect of phenothiazines on reaction time in schizophrenics. J.Psychiat. Res. $7,209-213$.

HELZER, J.E., BROCKINGTON, I.F . and KENDELL, R.E. (1981) Predictive validity of DSM-III and Feighner definitions of schizophrenia. A comparison with Research Diagnostic Criteria and Catego. Arch. Gen. Psychiatry $38,791-797$.

HEMSLEY, D.R. (1977) What have cognitive deficits to do with schizophrenic symptoms? Brit.J.Psychiat. 130, 167-173.

HERMAN, J., MIRSKY, A.F., RICKS, N.L. and GALLANT, D. (1977) Behavioral and alectrographic measures of attention in children at risk for schizophrenia. J.Abnorm.Psychol. B6, 27-33.

HILLYYARD, S.A. (1973) The CNV and human behavior. A review. In: W.C. MoCallum and J.R. Knott (Eds.) Event-related slow potentials of the brain: their relations to behavior. Electroenceph.clin. Neurophysiol., Suppl. 33.

HILLYARD, S.A., SQUIRES, K.C., BAUER, J.W. and LINDSAY, P.H. (1971) Evoked potential correlates of auditory signal detection. Science $172,1357-1360$.

HINTON, J. and CRASKE, B. (1976) A relationship between Eysenck's $P$ scale and change in muscle action potentials $v i$ th attention to perceptual tasks. Br.J.Psychol. 67, 461-466. 
HIRSCH, B.J. (1981) Coping and adaptation in high-risk populations: Toward an integrative model. Schizophrenia Bul1. 7, 164-172.

HOCKEY, R. (1979) Stress and the cognitive components of skilled pierformance. In: $V$. Hamilton and D. M. Warburtan (Eds.) Human stress and cognition. An information processing approach. Chicester, Wiley.

HOLLISTER, L.E. (1970) Choice of antipsychotic drugs. Am.J.Psychiat. $127,104-108$.

HOLZMAN, P.S. (1975) Smooth-pursuit eye movements in schizophrenia: recent findings. In: D.X. Freedman (Ed.) Biology of the major psychoses. Res.Publ.Assoc.Res.Nerv.Ment.Dis., Wol. 54. New York, Raven Press.

HOLZMAN, P.S., KRINGLEN, E., LEVY, D.L. and HABERMAN, S.J. (1980) Deviant eye tracking in tuins discordant for psychosis. Arch.Gen. Psychiatry $37,627-631$.

HOLZMAN, P.S., KRINGLEN, E., LEVY, D.L. , PROCTOR, L.R. and HABERMAN, S. (1978) Smooth pursuit eye movements in twing discordant for schizophrenia. J.Psychiat.Res. 14, 11-120. Also in: S. Matthijsse, B.J. Spring and J. Sugarman (Eds.) Attention and information processing in schizophrenia. Oxford, Pergamon, 1979.

HOLZMAN, P.S., KRINGLEN, E., LEWY, D.L., PROCTOR, L.R., HABERMAN, S.J. and YASILLO, N.J. (1977) Abnormal-pursuit eye movements in schizophrenia. Evidence for a genetic indicator. Arch.Gen.Psychiatry 34, 802-805.

HOLZMAN, P.S. and LEVY, D.L. (1977) Smooth pursuit eye movements and functional psychoses; a review. Schizophrenia Bull. 3, 15-27.

HOLZMAN, P.S., LEVY, D.L. and PROCTOR, L.R. (1976) Smooth pursuit eye movements, attiention, and schizophrenia. Arch. Gen.Psychiatry 33, $1415-1420$.

HOLZMAN, P.S., LEVY, D.L. and PROCTOR, L.R. (1978) The several qualities of attention in schizophrenia. J.Psychiat. Res. 14, 99-110. Also in: 5. Matthijsse, B.J. Spring and J. Sugarman (Eds.) Attention and information processing in schizophrenia. Oxford, Porganon, 2979.

HOLZMAN, P.S., LEVY, D.L., UHLENHUTH, E.H., PROCTOR, L.R. and FREEDMAN, D.X. (1975) Smooth pursuit eye movements, and Diazepam, CPZ, and Secobarbital. Psychopharmacologia (Ber1.) 44, 111-115.

HOLZMAN, P.S. "PROCTOR, L.R. and HUGHES, D.W. (1973) Eye-tracking patterns in schizophrenia. Science 181, 179-181.

HOLZMAN, P.S., PROCTOR, L.R., LEVY, D.L., YASILLO, N.J., MELTZER, H.Y. and HURT, S.W. (1974) Eye-tracking dysfunctions in schizophrenic patients and their relatives. Arch.Gen.Psychiatry 31, 143-151. 
MOVT, W.F * and DARDFF, A.B. (1971) Supranuclear disarders of ocular control systems in man. Clinical, anatomical, and physiological

correlations - 2969. In: P. Bach-y-Rita and C.C. Collins (Eds.) The control of eye movementa. New York, Academic Press.

HOYT, W.F. and FAISEN, L. (1975) Supranuclear ocular motor control. Some clinical considerations - 1974. In: G. Lennerstrand and P. P. Bach-y-Rita (Eds.) Basic mechanisms of ocular motility and their clinical implications. Oxford, Pergamon.

HUBER, G., GROSS, G. and SCHÜTTLER, R. (1979) Schizophrenie. Eine verlaufs- und sozial-psychiatriache Langzeitstudie. Berlin, Springer.

HUEER, G., GROSS, Gi, SCHÜTILER, R. and LINZ, M. (1980) Longitudinal studies of schizophrenic patients. Schizophrenia Bul1. 6, 592-605.

IACONO, W.G. (1978) Individual differences in smooth pursuit and saccadic eye movements and manual tracking performance in monozygotic twins: some implications for schizophrenia. Dissertation. Abstract in: Schizophrenia Bull. 6, 152.

IACONO, W.G. and LYKKEN, D.T. (1979-a) Electro-oculographic recording and scoring of smooth pursuit and saccadic eye tracking: a parametric study using monozygotic tuins. Psychophysiol. 16, 94-107.

IACONO, W.C. and LYKKEN, D.T. (1979-b) Eye tracking and psychopathology. New procedures applied to a sample of normal monozygotic twins. Arch.Gen.Paychiatry 36, 1361-1369.

IACONO, W.G. and LYKKEN, D.T. (1979-c) Comments on 'Smooth pursuit eye movements: a comparison of two measurement techniques' by Lindsey, Holzman, Haberman and Yasillo. J.Abnorm.Psychol. 8iB, 678-680.

IACONO, W.G. and LYKKEN, D.T. (19BI) Tuo-year retest stability of eye tracking performance and a comparison of electro-oculagraphic and infrared recording techniques: evidence of EEG in the ellectroaculogram. Psychophysiol. 18, 49-55.

IACOND, W.G., TUASON, V.B. and JOHNSON, R.A. (1981) Dissociation of smooth-pursult and saccadic eye tracking in remitted schizophrenics. An acular reaction time task that schizophrenics perform well. Arch. Cen. Psychiatry 38, 991-996.

IVERSEN, L.L. (1978) Biochemical and pharmacological studies: the dopamine hypothesis. In: J.K. Wing (Ed.) Schizophrenia. Tawards a new synthesis. London, Acadenic Press.

JACOB, T. (1975) Family interaction in disturbed and normal families: A methodological and substantive review. Psychol. Buld. 18, 35-65.

JAMES, W. (1950) The principles of psychology. New York, Dover. (Originally published in 1890). 
JANSSEN, R.H.C. (1973) Psychoticisme als persoonlijkheidsvariabele. Researchrapport $\mathrm{Nr}$. 7302 , Afdeling Electroencephalografie en Psychofysiologie. Psychiatrische Kliniek, Rijksuniversiteit Leiden.

JANSSEN, R.H.C. , MATTIE, H., PLDOIJ-VAN GORSEL, P.C. and WERRE, P.F. (1978) The effects of a depressant and a stimulant drug on the contingent negative variation. Biol. Psychol. 6, 209-218.

JÄRV ILEHTD, T. and FRUHSTORFER, H. (1970) Differentiation between slow cortical potentials associated with motor and mental acts in man. Exp.Brain.Res. 11, 309-3i7.

JERIS[N, H.J. (1970) Vigilance, discrimination, and attention. In: D.I. Mostofsky (Ed.) Attention: contemporary theory and analysis. New York, Appleton-Century-Crofts.

JOHN, E.R., KARMEL, B.Z., CORNING, W.C., EASTON, P., BROWN, D., AHN, H., JOHN, M., HARMDNY, T., PRICHEP, L., TORO, A , GERSON, I., BARTLETT, F., THATCHER, R., KAYE, H., VALDES, P. and SCHMARTZ, E. (1977) Neurometrics. Numerical taxonomy identifies different profilles of brain functions within groups of behaviorally similar people. Science 196, 1393-1410.

JOHNSTONE, E.C., FRITH, C.D., GOLD, A. and STEVENS, M. (1979) The outcome of severe acute schizophrenic illnesses after one year. Brit. J.Psychiat. 134, 28-33.

KAHNEMAN, D. (1973) Attention and effort. Englewood Cliffs, Prentice-Hall.

KAMPEN, D. van (1976) De "personality questionnaire" van Eysenck and Eysenck: een factoranalytisch onderzoek. Ned.T.Psychol. 31, 23-34.

KENDELL, R.E. (1975) The role of diagnosis in psychiatry. Oxford, Blackwell Scientific Publications.

KENDELL, R.E., PICHOT, P. and VON CRANACH, M. (1974) Diagnostic criteria of English, French and Cerman psychiatrists. Psychol.Med. 4, 1.87-195.

KENDLER, K.S., GRUENBERG, A.M. and STRAUSS, 3.5. (1981) An independent analysis of the Copenhagen sample of the Danish adoption study of schizophrenia. II. The relationship between schizotypal personality disorder and schizophrenia. Arch. Gen.Psychiatry 38, 982-984.

KERNBERG, 0. (1967) Borderline personality organization. J.Am.Paycho-anal. Assoc. 15, 641-685.

KETY, S.5., ROSENTHAL, D., WENDER, P.H. and SCHULSINGER, F. (1968) The types and prevalence of mental illness in the biological and adoptive families of adopted schizophrenics. In: D. Rosenthal and S.S. Kety (Eds.) The transmission of schizophrenia. Elms ford, N.Y., Pergamon Press. 
KETY, S.S., ROSENTHAL, D., WENDER, P.H. and SCHULSINGER, F. (1971) Mental 11 neas in the biological and adoptive famlies of adopted schizophirenics. Am.J.Paychiat. 128, 302-306.

KIETZMAM, M.L., SPRING, B. and ZUBIN, J. (1980) Perception, cognition and attention. In: H.I. Kaplan, A.M. Freedman and B.J. Sadock (Eds.) Comprehensive textbook of paychiatry, Third edition. Baltimore, Williams and Wilkins.

KING, H.E. (1975) Psychomotor correlates of behavior disorder. In: M.L. Kietzman, S. Sutton and J. Zubin (Eds.) Experimental approaches to paychopathology. New York, Academic Press.

KINZLER, E* (1971) Erfahrungen mit dem Depotneuroleptikum Dapotum D. Therapieuroche $21,2672-2677$.

KLEIN, R.H., SALZMAN, L.F., JDNES, F* and RITZLER, B. (1976) Eye tracking in poychiatric patients and their offspring (Abstract). Psychophysiol. 13, 186.

KLORMAN, R., THOMPSON, L.W. and ELLINGSON, R.J. (1978) Event-related brain potentials across the life span. In: E.P. Callaway, P. Tueting and S.H. Koslow (EdB.) Event-related brain potentials in man. New York, Academic Press.

KNIGHT, R. (1953) Borderline states. Bull.Menninger Clin. 17, 1-12.

KNIGHT, R.A., RIFF, J.D., BARRNETT, J. and MOSS, J.L. (1979)

Concurrent and predictive validity of thought disorder and affectivity: a 22-year follow-up of acute schizophrenics. J.Abnorm. Psycho1. 88, 1-12.

KNOTT, J.R. and IRWIN, D.A. (1967) Anxiety, stress, and the contingent negative variation. Electroenceph.clin. Meurophysiol. 22, 188.

KNNOTT, J.R. and IRWIN, D.A. (1968) Anxiety, stress, and the contingent negative variation (CNV). Electroenceph.clin. Neurophysiol. 24, 286-287.

KOHN, M.L. (1973) Social clase and schizophrenia: a critical review and reformuletion. Schizophrenia Bul1. 1 (7), 60-79.

KOHN, M.L. (1976) The interaction of social class and other factors in the etiology of schizophrenia. Am.J.Psychiat. 133, 177-180.

KOKES, R.F., STRAUSS, J.S. and KLORMAN, R. (1977) Premorbid adjustment in achizophrenia. Part. II. Measuring premorbid adjustment: the instruments and their development. Schizophrenia Bul1. 3, 186-213.

KOPFSTEIN, J.H. and NEALE, J.H. (1972) A multivariate study of attention dysfunction in schizophrenia. J.Abnorm.Psychol. 80, 294-298. 
KOPFSTEIN, J.H. and NEALE, J.M. (1973) Performance profiles of hospitalized psychiatric patients. Percept.mot.Skills 36, 739-744.

KORBOOT, P.J. and DAMIANI, N. (1976) Auditory processing speed and signal detection in schizophrenia. J.Abnorm. Psychol, 85, 287-295.

KORNETSKY, C. (1972) The use of a simple test of attention as a measure of drug effects in schizophrenic patients. Paychopharmacologia (Ber1.) 24, 99-106.

KRAEPELIN, E. (1896) Psychiatrie. Se Auflage. Leipzig, Verlag von Johann Ambrosius Barth.

KRAEPELIN, E. (1904) Psychiatrie. 7e Auflage, II Band. Leipzig, Verlag von Johann Ambrosius Barth.

KUDA, K. (1977) The effect of diazepan, chlorpromazine and amobarbital on the contingent negative variation. Folia Psychiat. Neurol.Jap. 31, $77-87$.

KLECHENMEISTER, C.A., LINTON, P.H., MUELLER, T.V. and WHITE, H.B. (1977) Eye tracking in relation to age, sex, and illnese. Arch.Gen. Psychiatry $34,578-579$.

KUHN, T.S. (1962) The structure of scientific revolutions. Chicago, The University of Chicago Press.

LANGFELDT, G. (1960) Diagnosis and prognosis in schizophrenia. Proc. Royal. Soc.Med. 53, 1047-1052.

LAPIDUS, L.B. and SCHMOLLING, P. (1975) Anxiety, arousal and schizophrenia* a theoretical integration. Psychol.Bu11. 82, 689-710.

LATHAM, C., HOLZMAN, P.S., MANSCHRECK, T.C. and TOLE, J. (1981) Dptokinetic nystagmus and pursuit eye movements in schizophrenia. Arch. Gen.Psychiatry 38, 997-1003.

LATZ, A. and KORNETSKY, C. (1965) The effects of chlorpromazine and secobarbital under two conditions of reinforcement on the performance of chronic schizophrenic subjects. Psychopharmacologia (Ber1.) 7 , $77-88$.

LAWSON, J.5., McIHIE, A. and CHAPMAN, J. (1966) Digtractibility in schizophremia and organic cerebral disease. Brit.J.Psychiat. 113, $527-535$

LEFF, J. (1978) Social and psychological causes of the acute attack. In: J.K. Wing (Ed.) Schizophrenia. Towards a new synthesis. London, Academic Press.

LEHMAN, H.E. (1975) Psychopharmacological treatment of schizopnrenia. Schizophrenia Bul1. 1 (13), 27-45. 
LEVIN, S. LIPTON, R.B. and HOLZMAN, P.S. (1981) Pursuit eye movements in psychopathology: Effects of target characteristics. Biol. Psychiat. 16, 255-267.

LEWINE, R.R.J., WATT, N.F., PRENTKY, R.A. and FRYER, J.H. (1980) Childhood social competence in functionally disordered psychiatric patients and in normals. J.Abnorm. Psychol. 89, 132-138.

LIDZ, T. (1973) The origin and treatment of schizophrenic disorders. New York, Basic Books.

LIEBOWITZ, M.R. (1979) Is borderline a distinct entity? Schizaphrenia Bull. 5, 23-38.

LIEM, J.H. (1980) Family studies of schizophrenia: An update and commentary. Schizophrenia Bull. 6, 429-455.

LINDSEY, D.T. HOLZMAN, P.S., HABERMAN, S. and YASILLD, N.J. (1978) Snooth-pursuit eye movements: a comparison of two measurement techniques for studying schizophrenia. J.Abnorm. Psychol. 87, 491-496.

LIPTON, R.B., FROST, L.A. and HOLZMAN, P.S. (1980) Smooth pursuit eye movements, schizophrenia, and distraction. Percept.mot.Skilis 50 , $159-167$.

LIPTON, R.B., LEVIN, S. and HOLZMAN, P.S. (1980) Horizontal and vertical pursuit eye movements, the oculocephalic reflex, and the functional psychoses. Psychiat.Res. 3, 193-203.

LONDON, N.J. (1973) An essay on psychoanalytic theory: two theories of schizophrenia. Part I: Review and critical assessment of the development of the two theories. Int.J.Psycho-Ana1. 54, 169-177.

LOVELESS, M.E. (1973) The contingent negative variation related to preparatory set in a reaction time situation with variable foreperiod. Electroenceph.clin. Neurophysiol. 35, 369-374.

LQVELESS, N.E, and SANFORD, A.J. (1974) Slow potential correlates of preparatoxy set. Biol. Psychol. 1, 303-314.

LOW, M.D. (1969) Discusaion of: Applications a la pathologie. In: J. Dargent et $M$. Dongier (Eds.) Variations contingentes négatives. Congrès et colloques de l"université de Liège, Vol. 52.

LOW, M.C., COATS, A.C., RETTIG, G.M. and MCSHERRY, J.W. (1967)

Anxiety, attentiveness-alertness: a phenomenological study of the CNV. Neuropsychologia $5,379-384$.

LUMSDEN, C.J. and WILSON, E.0. (1981) Genes, mind, and culture. The coevolutionary process. Cambridge, Massachusetts, Harvard University Press. 
LURIA, A.R. (1980) Higher cortical functions in man. Second Edition, Revised and Expanded (Authorized translation from the Russian). New York, Basic Books.

LURIA, R.E. and McHUGH, P.R. (1974) Reliability and clinical utility of the 'Wing' Present State Examination. Arch. Gen.Psychiatry 30, $866-871$.

LYKKEN, D.T., IACONO, W.G. and LYKKEN, J.D. (1981) Measuring deviant eye tracking. Schizophrenia Bull. 7, 204-205.

LYKKEN, D.T., TELLEGEN, A. and DERUBEIS, R. (1978) Volunteer bias in twin research: the rule of two-thirds. Soc.Biol. 25, 1-9.

MARSHALL, W.L. (1973) Cognitive functioning in schizophrenia. 1. Stimulus analysing and response selection processes. Brit..J.Psychiat. $123,413-423$.

MAY, H.J. (1979) Oculomotor pursuit in schizophremia. Arch.Gen. Psychiatry $36,827$.

MCCALLUM, W.C. (1969) The contingent negative variation as a cortical sign of attention in man. In: Evans and Mulholland (Eds.) Attention in neurophysiology. London, Butterworth.

MCCALLUM, W.C. (1973) The CNV and conditionability in psychopaths. In: W.C. McCallum and J.R. Knott (Eds.) Event-related slow potentials of the brain: their relations to behavior. Electroenceph.clin. Neuraphysiol., Suppl. 33.

MCCALLUM, W.C. (1979) Cognitive aspects of slow potential changes. In: J.E. Desmedt (Ed.) Cognitive components in cerebral event-related potentials and selective attention. Prog.clin.Neurophysiol., Vol. 6. Basel, Karger.

MCCALLUM, W.C. and ABRAHAM, P. (1973) The contingent negative variation in psychosis. In: W.C. McCallum and J.R. Knott (Eds.) Event-related slow potentials of the brain: their relations to behavior. Electroenceph.clin. Neurophysiol., Suppl. 33.

MCCALLUM, W.C. and CURRY, S.H. (1981) Late slow wave components of auditory evoked potentials: their cognitive significance and interaction. Electroenceph.clin. Neurophysiol. 51, 123-137.

MCCALLUM, W.C. and PAPAKOSTOPDULOS, D. (1973) The CNV and reaction time in situations of increasing complexity. In: W.C. McCallum and J.R. Knott (Eds.) Event-related slow potentials of the brain: their relations to behavior. Electroenceph.clin.Neurophysiol., Suppl. 33.

MCCALLUM, W.C. and WALTER, W.G. (1968-a) The differential effects of distraction on the contingent negative variation in normal subjects and psychiatric patients. Electroenceph.clin. Neurophysiol. 24, 593. 
MCCALLUM, W.C. and HALTER, W.G. (1968-b) The effects of attention and distraction on the contingent negative variation in normal and neurotic patients. Electroenceph.clin.Neurophysiol. 25, 319-329.

MacDONALD, N. (1960) Living with schizophrenia. Can.Med.Ass.J. 82, 218-221. Also in: B. Kaplan (Ed.) The inner vorld of mental illness. A series of first-person accounte of what it was like. New York, Harper and Roy Publishers, 1964.

MCDOUCALL. W. (1905) On a new method for the study of concurrent mental operations and of mental fatique. Brit.J.Psychol. 1, 435-445.

McGHIE, A. (1970) Attention and perception in sichizophrenia. In: B.A. Maher (Ed.) Progrese in experimental personality research, Vol* 5. New York, Academic Press.

MCGHIE, A. and CHAPMAN, J. (1961) Disorders of attention and perception in early schizophrenia. Brit.J.Med.Psychol. 34, 103-116.

MCGHIE, A., CHAPMAN, J. and LAWSON, J.5. (1965) The effect of distraction on schizophrenic perfornance: (1) Perception and immediate memory. Brit.d.Psychiat. 111, 383-390.

MCPHERSON, F.M., PRESLEY, A.5., ARMSTRONG, J. and CURTIS, R.H. (1974) "Peychoticism' and psychotic illness. Brit.J.Psychiat. 125, 152-160.

McSHERRY, J.W. (1973) Physiological origins. A review. In: W.C. McCallum and J.R. Knott (Eds.) Event-related slow potentials of the brain: their relations to behavior. Electroenceph.clin. Neurophysiol., Supp1. 33.

MEDNICK, S.A. (1958) A learning theory approach to research in schizophrenia. Psychal.Bull. 55, 315-327.

MEEHL, P.E. (1962) 'Schizataxia, schizotypy, schizophrenia'. Am. Psychol. 17, 827-838.

MELTZER, H.Y. (1979) Biochemical studies in schizophrenia. In: L. Bellak (Ed.) Disorders of the schizophrenic syndrame. New York, Basic Books.

MELTZER, H.Y. and STAHL, S. (1976) The dopamine hypothesis: a review. Schlzophrenia Bul1. 2, 19-76.

MENNINGER, K., MAYMAN, M. and PRUYSER, P. (1963) The vital balance: the Iife process in mental health and illness. New York, Viking Press.

MEYER, A. (1951) Fundamental conceptions of dementia praecox. In: Collecteid works, Vol. 2. Baltimore, John Hopkins Press.

MIALET, J1.P. and PICHOT, P. (1981) Eye-tracking patterns in schizophrenia. An analysis based on the incidence of saccades. Arch.Gen.Psychiatry 38, 183-186. 
MILLER, J.G. (1978) Living systems. New York, McGrau-Hil1.

MIRSKY, A.F. and KORMETSKY, C. (1964) On the dissimilar effects of drugs on the Digit Symbol Substitution and Continuous Performance Tests. Psychopharmacalogia (Ber1.) 5, 161-177.

MOL, J.M.F., CABERG, H.B. and ROZENDAAL, N. (1982) Pseudo D.C. restauratie van evoked responsies die $z$ ijn opgenomen met een korte tijjconstante. Paper read at the Ned. Vereniging v. Klin. Neurofysiologie, Utrecht.

NAATANEN, R. and GAILLARD, A.W. (1974) The relationship between the contingent negative variation and the raction time under prolonged experimental conditions. Biol.Psychol. 1, 277-291.

NEALE, J.M. and CROMWELL, R.L. (1970) Attention and schizophrenia. In: B.A. Maher (Ed.) Progress in experimental personality research, Vol. 5. New York, Academic Press.

NEALE, J.M. and OLTMANNS, T.F. (1980) Schizophrenia. New York, Willey.

NEWLIN, D.B., CARPENTER, B. and GOLDEN, C.J. (1981) Hemispheric asymmetries in schizophrenia. Biol.Psychiat. 16, 561-582.

NIDEFFER, R.M., NEALE, J.M. , HELD, J., KOPFSTEIN, M.A. and CROMWELL, R.L. (1971) The effect of previous preparatory intervals upon anticipatory responses in the reaction time of schizophrenic and nonschizophrenic patients. J.Nerv.Ment.Dis. 153, 360-365.

NISSEN; M.J. (1977) Stimulus intensity and information processing. Percept.Psychophysics 22, 338-352.

NORMAN, D.A. (1976) Memory and attention. An introduction to human information processing. Second edition. New York, Wiley.

NORMAN, D.A. and BOBROW, D.G. (1975) On data-limited and resourcelimited processes. Cognit.Psychol. 7, 44-64.

NUECHTERLEIN, K.H. (1977) Reaction time and attention in schizophrenia: a critical evaluation of the data and theories. Schizophrenia Bull. 3, 373-428.

OBERSTEINER, $H$ : (1874) Ueber eine neue einfache Methode zur Beatimmung der psychischen Leistungsfähigkeit des Gehirnes Geisteskranker. Vilirchous Arch. 59, 427-458.

QLBRICH, R. (1972) Reaction time in brain-damaged and normal subjects to variable preparatory intervals. J.Nerv.Ment.Dis. 155, 356-362.

ORZACK, M.H. and KORNETSKY, C. (1966) Attention dysfunction in chronic schizophrenia. Arch. Gen.Psychiatry 14, 323-326. 
ORZACK, M.H. and KORNETSKY, C. (1971) Environmental and familial predictors of attention behavior in chronic schizophrenics.

J.Psychiat. Res. 9, 21-29.

ORZACK, M.H., KORNETSKY, C, and FREEMAN, H. (1967) The effects of daily adrinistration of carphenazine on attention in the schizophrenic patient. Psychopharnacologia (Ber1.) 11, 31-38.

OUERALL, J.E. and HOLLISTER, L.E. (1979) Camparative evaluation of research diagnostic criteria for schizophrenia. Arch. Gen.Psychiatry 36, $1198-1205$.

PAPAKOSTOPOULOS, D. and FENELON, B. (1975) Spatial distribution af the contingent negative variation (CNV) and the relationship between CNV and reaction time. Psychophysiol. 12, 74-78.

PASS, H.L., KLQRMAN, R., SALZMAN, L.F., KLEIN, R.H. and KASKEY, G.B. (1980) The late positive component of the evoked response in acute schizophrenics during a test of sustained attention. Biol. Psychiat. $15,9-20$.

PASS, H.L., SALZMAN, L.F., KLORMAN, R., KASKEY, G.B. and KLEIN, R.H. (1978) The effect of distraction on acute schizophrenics" visual tracking. Biol. Psychiat. 13, 587-593.

PAYNE, R.W. (1966) The measurement and significance of overinclusive thinking and retardation in schizophrenic patients. In: P. Hoch and J. Zubin (Eds.) Psychopathology of schizophrenia. New York, Grune and Stratton.

PAYNE, R.W. (1971) Cognitive defects in schizophrenia: overinclusive thinking. In: J. Helmuth (Ed.) Cognitive studies, Vol. 2. Deficits in cognition. New York, Brunner/Mazel.

PAYNE, R.W. and HEWLETT, J.H.G. (1960) Thought disorder in psychatic patients. In: H.J. Eysenck (Ed.) Experiments on personality, Vol. 2. London, Rautledge and Kegan Paul.

PAYNE, R.W., HOCHBERG, A.C. and HAWKS, D.V. (1970) Dichotic stimulation as a method of assessing disorder of attention in overinclusive schizaphrenic patients. J.Abnarm. Peychol. 76, 185-193.

PERRY, J.C. and KLERMAN, G.L. (1970) The borderline patient. A comparative analysis of four sets of diagnostic criteria. Arch. Gen. Psychiatry $35,141-150$.

PETERS, J.F. and KNOTT, J.R. (1976) CNV and post-response negativity with stressful auditory feedback. In: W.C. MeCallum and J.R. Knott (Eds.) The responsive brain. Bristol, John wight and Sons.

PHILPOTT, S.J.F. (1932) Fluctuations in human output. Brit.J.Psychol., Monog. Suppl. 17. 
PHILPOTT, S.J.F. (1934) A theoretical curve of fluctuations of attention. Brit. J.Psychol. 25, 221-255.

PILLSBURY, W.B. (1908) Attention. London, Swan Sonnenschein.

PIVIK, R.T. (1979-a) Smooth pursuit eye movements and attention in psychiatric patients. Biol.Psychiat. 14, 859-879.

PIUIK, R.T. (1979-b) Target velocity and smoath pursuit eye movements in psychiatric patients. Psychiat. Res. 1, 313-323.

PLOOIJ-UAN GORSEL, P.C. (1980) Persoonlijkheid en arousal. Een onderzoek naar corticale en autonome funkties in relatie tot persoonlijkheid. Dissertation. Lisse, Swets en Zeitlinger.

POPE, H.G. and LIPINSKI, J.F. (1978) Diagnosis in schizophrenia and manic-depressive illness. Arch.Gen.Psychiatry 35, 811-828.

POSNER, M.I. (1975) Psychobiology of attention. In: M.S. Gazzaniga and C. Blakemore (Eds.) Handbook of psychobiology. New York, Academic Press.

POSNER, M.I. and BOIES, S.W. (1971) Components of attention. Psychol.Rev. 78, 391-408.

POSNER, M.I., KLEIN, R., SUMMERS, J. and BUGGIE, S. (1973) On the selection of signals. Memory.Gognit. 1, 2-12.

PROCCI, W.R. (1976) Schiza-affective psychosis: fact or fiction? Arch.Gen.Psychiatry 33, 1167-1178.

RASHBASS, C. (1961) The relationship between saccadic and amooth tracking eye movements. J.Physiol. (Lond.) 159, 326-338.

REBERT, C.S., MCADAM, D.W. KNOTT, J.R. and IPWIN, D.A. (1967) Slow potential change in human brain related to level of motivation. J. comp.physiol.Psychol. 63, 20-23.

REBERT, C.S. and TECCE, J.J. (1973) A sumnary of CNV and reaction time. In: W.C. McCallum and J.R. Knott (Eds.) Event-related potentials of the brain: their relations to behavior. Electroenceph.clin. Neurophysiol., Suppl. 33.

REICH, W. (1976) The schizophrenia spectrum: a genetic concept. J. Nerv.Ment.Dis. 162, 3-12.

RDBINS, L.N., HELZER, J.E., CROUGHAN, J. and RATCLIFF, K.S. (1981) National Institute of Mental Health Diagnostic Interview. Its history, characteristics, and validity. Arch.Gen.Psychiatry 38, 381-389.

ROBINSON, D.A. (1971) Models of oculomotor neural organization. In: P. Bach-y-Rita and C.C. Collins (Eds.) The control of eye movementa. New York, Academic Press. 
RODNICK, E.H. and SHAKDW, D. (1940) Set in the schizophrenic as measured by a composite reaction time index. Am.J.Psychiat. 97, 214-225.

ROSE, 5. (1976) The conacious brain. Revised edition. Harmondsworth, Penguin Booka.

FOSENTHAL, D. (1974) The concept of subachizaphrenic disorders. In: S.A. Mednick, F. Schulsinger, J. Higgins and B. Bell (Eds.) Genetics, environment and psychopathology. Amsterdam/New York, North Holland/ American Elsevier.

ROSENTHAL, D. (1975) The spectrum concept in schizophrenic and manic-depressive disorders. In: D.X. Freedman (Ed.) Biology of the major psychoses. Res.Publ.Assoc.Res.Nerv.Ment.Dis., Vol. 54. New York, Raven Press.

ROSENTHAL, D. (1978) The schizophrenia spectrum disiorders:

implications for psychiatric diagnosis. In: H.G. Akiskal and W.L. Webb (Eds.) Psychiatric diagnosis: exploration of biological predictors. New York, Spectrum Publications.

ROSENTHAL, D., WENDER, P.H., KETY, S.S., WELNER, ]. and SCHULSINGER, F. (1971) The adopted-away offspring of schizophrenics. Arch. Gen. Psychiatry 128, 307-311.

ROSWOLD, H.E., MIRSKY, A.F., SARASON, I., BRAMSOME, E.D. and BECK, L.H. (1956) A continuous performance test of brain damage. J. Consult. Psychol. 20, 343-350.

RUMKE, H.C. (1943) Het kernsymptoom der schizophrenie en het "praecoxgevoel'. In: Studies en voordrachten over paychiatrie. Amsterdam, Scheltema en Holkema.

RUTSCHMANN, J., CORNBLATT, B. and ERLENMEYER-KIMLING, L. (1977) Sustained attention in children at risk for schizophrenia. Arch.Gen. Paychiatry $34,571-575$.

SACCUZZO, D.P., and SCHUBERT, D.L. (1981) Backward masking as a measure of slow processing in schizophrenia spectrum disorders. J.Abnorm. Paychol. $90,305-312$.

SALETU, B. (1974) Classification of psychatropic druge based on human ovoked potentials. In: T.M. Itil (Ed.) Psychotropic drugs and the human EEG. Mod.Probl. Pharmacopsychiat., Vol. 8. Basel, Karger.

SALZMAN, L.F., KLEIN, R.H. and STRAUSS, J.S. (1979) Pendulum eye-tracking in remitted psychiatric patiente. J.Psychiat.Res. 14, 121-126. Also in: S. Matthijsee, B.J. Spring and J. Sugarman (Eds.) Attention and information processing in schizophrenia. Oxford, Pergamon, 1979. 
SCHEFF, T.J. (1966) Being mentally ill, a sociological theory. Chicago, Aldine.

SCHEFF, T.J. (1970) Schizophrenia as ideology. Schizophrenia Bull. I (1), 15-20. Also in: T.J. Scheff (Ed.) Labeling madness. Englewood Cliffe, Prentice-Hall, 1975.

SCHNEIDER, K. (1962) Klinische Psychopathologie. Ge Auflage. Stuttgart, Georg Thieme Verlag.

SCHNEIDER, W. and SHIFFRIN, R.M. (1977) Controlled and autonatic information processing: $I$. Detection, search and attention.

Psychol. Rev. 84, 1-66.

SCHWARTZ, C.C., MYERS, J.K. and ASTRACHAN, B.M. (1975) Concordance of multiple assessments of the outcome of schizophrenia. On defining the dependent variable in outcome studies. Arch. Gen.Psychiatry 32, $1221-1227$.

SHAGASS, C. (1974) Effects of psychotropic drugs on human evoked potentials. In: T.M. Itil (Ed.) Psychotropic drugs and the human EEG. Mod.Probl.Pharmacopsychiat., Vol. 8. Basel, Karger.

SHAGASS, C., AMADEO, M. and OVERTON, D.A. (1974) Eye-tracking performance in psychiatric patients. Biol. Psychiat. 9, 245-260.

SHAGASS, C., ROEMER, R.A. and AMADEO, M. (1976) Eye-tracking performance and engagement of attention. Arch.Gen.Psychiatry 33, 121-125.

SHAGASS, C. and STRAUMANIS, J.J. (1978) Drugs and human sensory evoked potentials. In: M.A. Lipton, A. DiMascio and K.F. Killam (Eds.) Psychopharmacology: a generation of progress. Nev York, Raven Press.

5HAKOW, D. (1962) Segmental set. Arch.Gen.Psychiatry 6, ]7-37.

SHAKOW, D. (1963) Psychological deficit in schizophrenia. Behav.Sci. B, 275-305.

SHAKOW, D. (1971) Some abservations on the psychology (and some fever, on the biology) of schizaphrenia. J.Nerv.Ment.Dis. 153, 300-316.

SHARPE, L., GURLAND, B.J., FLEISS, J.L., KENDELL, R.E., COOPER, J.E. and COPELAND, J.R.M. (1974) Some comparisons of American, Canadian and British psychiatrists in their diagnostic concepts. Can.Psychiat. Ass. J. $19,235-245$.

SHIFFRIN, R.M. and SCHNEIDER, W. (1977) Controlled and automatic human information processing: II. Perceptual learning, automatic attending, and a general theory. Psychol.Rev. 84, 127-190.

SIEBERNS, S. (1978) Erfahrungen mit dem Depotneuroleptikurn Flupentixoldekanoat - Eine Ubersicht. Pharmakopsychiat. 11, 186-198. 
SIEUER, L.J. and GUNDEPSON, J.G. (1979) Genetic determinants of baiderline conditions. Schizophrenia Bul1. 5, 59-86.

SILVENMAN, J. (1964) The problem of attention in research and theory in schizophrenia. Psychol. Rev. 71, 352-379.

SKINNER, H.A. (1981) Toward the integration of classification theory and methods. J.Abnorm.Psychol. 90, 68-87.

SHALL, J.G. and SMALL, I.F, (1971) Contingent negative variation (CNV). Correlations with psychiatric diagnosis. Arch.Gen.Psychiat. 25, $550-554$.

SNEATH, P.H.A. and SDKAL, R.R. (1973) Numerical taxonomy. The principles and practice of numerical classifications. San Francisco, H. H. Feenan and Company.

SOKAL, R.R. and SNEATH, P.H.A. (1963) Principles of numerical taxonomy. San Francisco, W.H. Freeman and Company.

SPITZER, R.L., ANDREASEN, N.C. and ENDICOTT, J. (1978) Schizophrenia and other psychotic disorders in DSM-III. Schizophrenia Bull. 4, 489-509.

SPITZER, R.L. and ENDICOTT, J. (1978) Schedule for Affective Disorders and Schizophrenia SADS. Third edition. New York, Biometrics Research, New York State Psychiatric Institute.

SPITZER, R.L. and ENDICOTT, J. (1979) Justification for separating schizotypal and borderline personality disorders. Schizophrenia Bull. 5, $95-104$.

SPITZER, R.L., ENDICOTT, J. and GIBBON, M. (1979) Crossing the border into borderline personality and borderline schizophrenia: the developiment of criteria. Arch.Gen. Psychiatry 36, 17-24.

SPITZER, R.L., ENDICOTT, J. and ROBINS, E. (1978-a) Research Diagnostic Criteria (ROC) for a selected group of functional disorders. Third edition. New York, Biometrics Research, New York state Paychiatric Institute.

SPITZER, R.L., ENDICOTT, J. and ROBINS, E. (1978-b) Research Diagnostic Criteria. Arch. Gen.Psychiatry 35, 773-782.

SPITZER, R.L., FORMAN, J.B.W. and MEE, J. (1979) DSM-III field trials: I. Initial interrater diagnostic reliability. Ar.J.Psychiat. 136 , $815-817$.

SPOHN, H.E. and FITZPATRICK, T. (1980) Informed consent and bias in samples of schizophrenic subjects at risk for drug withdrawal. J. Abnorm. Psychol. 199, 79-92. 
SPOHN, H.E., LACOURSIERE, R.B., THOMPSON, K. and COYNE, L. (1977) Phenothiazine effects on psychological and psychophysiological dysfunction in chronic schizophrenics. Arch.Gen.Psychiatry 34 , $633-644$.

SPRING, B.J. and ZUBIN, J. (1978) Attention and information processing as indicators of vulnerability to schizophrenic episodes. J.Psychiat. Res. 14, 289-301. Also in: S. Matthijsse, B.J. Spring and J. Sugernan (Eds.) Attention and information processing in schizophrania. Oxford, Pergamon, 1979.

STEINMAN, R.M. (1975) Dculomotor effects on vision. In: G. Lennerstrand and P. Bach-y-Rita (Eds.) Basic mechanisms of ocular motility and thejr clinical implications. Dxford, Pergamon.

STEFFY, R.A. (1978) An early cue sometimes impairs pracess schizophrenic performance. J.Psychiat. Res. 14, 47-57. Also in: S. Matthijsse, B.J. Spring and J. Sugarman (Eds.) Attention and information processing in schizophrenia. Oxford, Pergamon, 1979.

STEFFY, R.A. (1980) Redundancy deficit in normal control subjects? Paper presented at the Annual Meetings of the Canadian Psychological Association, Calgary, Alberta.

STEFFY, R.A. and GALBRAITH, K. (1974) A comparison of segmental set and inhibitory deficit explanations of the crossover pattern in process schizophrenic reaction time. J.Abnorm. Psychol. 83, 227-233.

STEFFY, R.A. and GALBRAITH, K. (1975) Time linked impairment in schizophrenic reaction time performance. J.Abnorm.Psychol. 84, 315-324.

STEFFY, R.A. and GALBRAITH, K.J. (1980) Relation between latency and redundancy-associated deficit in schizophrenic reaction time performance. J.Abnorm. Psycha1. 89, 419-427.

STEFFY, R.A., MacCRIMMDN, D.J. and BELLISSIMO, A. (1979) Reaction time indices reflecting changes in the clinical state of chronic schizophrenic patients. Unpublished manuscript.

STRAUSS, M.E., BOHAUNON, W.E., KAMINSKY, M.J. and KHARABI, F. (1979) Simple reaction time crossover accurs in schizophrenic autpatienta. Schizophrenia Bu11. 5, 612-615.

STRAUSS, J.S. and CARPENTER, W.T. (1974) Characteristic symptoms and outcome in schizophrenia. Arch. Gen.Psychiatry 30, 429-434.

STRAUSS, J.S. and CARPENTER, W.T. (1977) Prediction of outcome in schizophrenia. III. Five-year outcome and its predictors. Arch.Cen. Psychiatry $34,159-163$. 
STRAUSS, J.S. and CARPENTER, W.T. (1978) The prognos is of achizophrenia: rationale for a multidimensional concept. Schizophrenia Bu11. 4, 56-67.

STRAUSS, J.S. and GIFT, T.E. (1977) Choosing an approach for diagnosing schizophrenia. Arch. Cen.Psychiatry 34, 1248-1253.

SLLLIVAN, H.5. (1953) Conceptions of madern psychiatry. Nev York, Norton.

SYNDULKD, K., PARKER, D.A., JENS, R., MALTZMAN, I. and ZISKIND, E. (1975) Psychophyaiology of sociopathy: electrocortical measures. Biol. Psychol. 3, 185-200.

SZASZ, T. (1976) Schizophrenia: the sacred symbol of psychiatry. Brit.J.Psychiat. 129, 308-316.

TATSUOKA, M.M. (1970) Discriminant analysis. The study of group differences. Selected topics in advanced statiatics. An elementary approach, Number 6. Champaign, Institute for Personality and Ability Testing.

TECCE, J.J. (1971) Contingent megative variation and individual differences. A new approach in brain research. Arch.Gen.Psychiatry 24, $1-16$.

TECCE, J.J. (1972) Contingent negative variation (CNW) and psychological processes in man. Psychol. Bul1. 77, 73-108.

TECCE, J.J. and COLE, J.0. (1974) Amphetamine effects in man: paradoxical drowsiness and lawered electrical brain activity (CNV). Science $185,451-453$.

TECCE, J.J., COLE, J.0. and SAVIGNANO-BOWMAN, J. (1975) Chlorpromazine effects on brain activity (contingent negative variation) and reaction time in normal wamen. Psychopharmacologia 43, 293-295.

TECCE, J.J., SAVIGNANO-BOWMAN, J. and MEINBRESSE, D. (1976) Contingent negative variation and the distraction-arousal hypothesis.

Electroenceph.clin. Neurophysiol. 41, 277-286.

TELLEGEN, A. (1978) Manual of the Differential Personality Questionnaire. University of Minnesota (unpublished).

TELLEGEN, A, and ATKINSON, G. (1974) Dpenness to absorbing and self-altering experiences ('absorption'), a trait related to hypnotic susceptibility. J.Abnorm. Poycho1. 83, 268-277.

TIMSIT-BERTHIER, M. (1976) La varjation contingente negative (VCN) et les maladies mentales. Acta psychiatr.belg. 76, 521-550.

TIMSIT-BERTHIER, M., DELAUNOY, J., KONINCKX, N. and ROUSSEAU, J.C. (1973) Slow potential changes in psychiatry. I. Contingent negative variation. Electroenceph.cIin. Neurophysiol. 35, 355-361. 
TIMSIT-BERTHIER, M., DELAUNOY, J. and ROUSSEAU, 3.C. (1973) S10w potential changes in psychiatry. II. Motor potential. Electroenceph. clin. Neurophysiol. 35, 363-367.

TIMSIT-BERTHIER, M. DELAUNOY, J. and ROUSSEAU, J.C. (1976) Some problems and tentative solutions to questions raised by slow potential changes in psychiatry. In: W.C. McCallum and J.R. Knott (Eds.) The responsive brain. Bristol, John Wright and Sons.

TIMSIT-BERTHIER, M. and GERONO, A. (1976) P 300 in psychotic patients. Poster presentation at the Fourth International Congress on Event-Related Slow Potentials of the Brain. Kanuga, N.C. Cited in: Roth, W.T. (1977) Late event-related potentials and psychopathology. Schizophrenia Bu11. 3, 105-120.

IIMSIT, M., KONINCKX, N., DARGENT, J., FONTAINE, O. et DONGIER, $M$. (1968) Etude de la variation contingente negative chez les psychotiques et les pré-psychotiques. Ann. Méd.-psychal. 1, 424-435.

TIMSIT, M. , KONINCKX, N., DARGENT, 3., FONTAINE, 0. et DONGIER, M. (1969) Etude de la durée des VCN chez un groupe de sujets normaux, un groupe de névrosés et un groupe de psychotiques. In: J. Dargent et: M. Dongier (Eds.) Variations contingentes negatives. Liège, Congrès et collaques de l'université de Liège, Vol. 52.

TIMSIT, M. , KONINCKX, N., DARGENT, J., FONTAINE, 0. et DONGIER, M. (1970) Variations contingentes négatives en psychiatrie.

Electroenceph.clin. Neurophysiol. 28, 41-47.

TIMSIT-BERTHIER, M. , KONINCKX, N., DELAUNOY, J. et ROUSSEAU, J.C. (1972) Nouvelles etudes etatistiques de la variation contingente négative en psychiatrie. Rev.EEG. Neurophsyiol. 2, 333-339.

TITCHENER, E.B. (1908) Lectures on the elementary psychology of feeling and attention. New York, MacMillan.

TIZARD, J. and VENABLES, P.H. (1956) Reaction time responses by schizophrenics, mental defectives, and normal adults. Am.J.Psychiat. $112,803-807$.

TOMER, R., MINTZ, M., LEVY, A. and MYSLOBODSKY, M. (1981) Smooth pursuit patterns in schizophrenics during cognitive task. Biol. Psychiat. 16, 131-144.

TREISMAN, A.M. (1964) Verbal cues, language and meaning in selective attention. Am. J.Psychol. 77, 215-216.

TREISMAN, A.M. (1969) Strategies and models of selective attention. Psychol. Rev. 76, 282-299.

TSUANG, M.T. and DEMPSEY, G.M. (1979) Long-term outcome of major psychoses. II. Schizoaffective disorder compared with schizophrenia, affective disorders, and a surgical control group. Arch. Gen.Psychiatry $36,1302-1304$. 
TSUANG, M.T. WOOLSON, R.F. and FLEMIMG, J.A. (1979) Long-tem outcome of major puychoses. I. Schizophrenia and affective disorders compared with psychiatrically sympton-free surgical conditions. Arch. Gen. Psychiatry 36, 1295-1301.

UNDERWOOD, G. (1978) Attentional selectivity and behavioural control. In: C. Underwood (Ed.) Strategies of information processing. London, Academic Press.

VAUGHN, C.E. and LEFF, J.P. (1976) The influence of family and social factors on the caurse of psychiatric illness. A comparison of schizophrenic and depressed neurotic patients. Brit.J.Psychiat. 129, 125-137.

VENABLES, P.H. (1963) The relationship between level of skin potential and fusion of paired light flashes in schizophrenic and normal subjects. J.Psychiat.Res. 1, 279-287.

VENABLES, P.H. (1964) Input dysfunction in schizophrenia. In: B.A. Maher (Ed.) Progress in experimental personality research, Val. 1. New York, Acadenic Press.

VENABLES, P.H. (1966) Psychophysiological aspects of schizophrenia. Brit.J.Med.Psychol. 39, 289-297.

VENABLES, P.H. (1975) Psychophysiological studies of schizophrenic pathology. In: P.H. Venables and M.J. Christie (Eds.) Research in psychophysiology. London, Wiley.

VEREBLY, K., WOLAUKA, J. and CLDUET, D. (1978) Endorphins in psychiatry: an overview and a hypothesis. Arch. Gen.Psychiatry 35 , $877-888$.

VERHEY, F.H.M. (1980) Langzame hersenpiotentialen en gemeten gedrag. Dissertation. Maastricht, Leiter-Nijpels.

VERMA, R.M. and EYSENCK, H.J. (1973) Severity and type of psychotic illness as a function of personality. Brit.J.Psychiat. 122, 573-585.

WALKER, E. (1981) Attentionai and neuromotor functions of schizophrenics, schizoaffectives, and patients with other affective disorders. Arch.Gen. Psychiatry 38, 1355-1358.

WALTER, W.G. (1966) Electrophysiologic contributions to psychiatric therapy. In: J.H. Masserman (Ed.) Current psychiatric therapies. New York, Grune and Stratton.

WALTER, W.G. (1967) Electrical signs of association, expectancy and decision in the human brain. Electroenceph. clin. Neurophysiol., Suppl. $25,258-263$. 
WALTER, W.G., COOPER, R., ALDRIDGE, W.J., MCCALLUM, W.C. and WINTER, A.L. (1964) Contingent negative variation: an electrical sign of sensory-motor association and expectancy in the human brain. Nature $203,380-384$.

WARBURTON, D.M. (1979-a) Physiological aspects of information processing and stress. In: V. Hamilton and D.M. Warburton (Eds.) Human stress and cognition. An information processing approach. Chicester, Wiley.

WARBURTON, D.M. (1979-b) Stress and the processing of information. In: V. Hamilton and D.M. Warburton (Eds.) Human stress and cogrition. An information processing approach. Chicester, Wiley.

WARD, C.H., BECK, A.T., MENDELSON, M., et a1. (1962) The psychiatric momenclature: Reasons for diagnostic disagreement. Arch. Cen.Psychiatry $7,198-205$.

WARD, J.H. (1963) Hierarchical grouping to optimize an objective function. J.Am. Stat.Assoc. 58, 236-244.

WEINER, H. (1980) Schizophrenia: etiology. In: H.I. Kaplan, A.M. Freedman and B.J. Sadock (Eds.) Comprehensive textbook of peychiatry, Third edition. Baltimore, Williams and Wilkins.

WEISS, A.D. (1965) The locus of reaction time change with set, motivation, and age. J.Gerontol. 20, 60-64.

WENDER, P.H. (1963) Dementia praecox: the development of the concept. Am. J.Psychiat. 119, 1143-1151.

WERRE, P.F., FAVEREY, H.A. and JANSSEN, R.H.C. (1975) contingent negative variation and personality. Ned.T.Psychol. 30, 277-299.

WILKINSON, R.T. and HAINES, E. (1970) Evoked response correlates of expectancy during vigilance. Acta pisychol. (Amst.), Suppl. 33, $402-413$

WILKINSON, R.T. and SEALES, D.M. (1978) EEG event-related potentials and signal detection. Biol. Psychal. $7,13-28$.

WILLIAMS, H.L., LUBIN, A. and GOOONOW, J.J. (1959) ImpaJired performance with acute sleep lass. Psychal.Mon. 79, 1-26.

WING, J.K. (1978) Social influences on the course of schizophrenia. In: L.C. Wynne, R.L. Cromwell and S. Matthijsse (Eds.) The nature of schizophrenia. New approaches to research and treatment. New York, Wiley.

WING, J.K., COOPER, J.E. and SARTORIUS, N. (1974) Measurement and classification of psychiatric symptoms. An instruction manual for the PSE and Catego program. Cambridge, Cambridge University Presa. 
WISHNER, J., STEIN, M.K. and PEASTREL, A.L. (1978) Information processing stages in schizophrenia. J.Psychiat.Res. 14, 35-45. Also in: S. Matthijsse, B.J. Spring and J. Sugarman (Eds.) Attention and information processing in schizophrenia. Oxford, Pergamon, 1979.

WOHLBEPG, G.W. and KORNETSKY, C. (1973) Sustained attention in remitted schizophrenics. Arch. Gen.Psychiatry 28, 533-537.

WORLD HEALTH DRGANIZATION (1973) Report of the International Pilot Study of Schizophrenia. Volume I. Geneva, WHO.

WDRLD HEALTH ORGANIZATION (1979) Schizophrenia. An international follow-up study. Chicester, Wiley.

WUNDT, W. (1874) Grundzüge der physiologischen Psychologie, Leipzig, Engelmann.

WYATT, R.J., POTKIN, S.G., BRIDGE, T.P., PHELPS, B.H. and WISE, C.D. (1980) Monoamine oxidase in schizophrenia: an overview. Schizophrenia Bull. 6, 199-207.

WWNNE, L.C., TOOHEY, M.L. and DOANE, J. (1979) Family studies. In: L. Bellak (Ed.) Disorders of the schizophrenic syndrome. New York, Basic Books.

WYNNE, L.C., RYCKOFF, I.M., DAY, J. and HIRSCH, S. (1958)

Pseudomutuality in the family relations of schizophrenics. Psychiatry $21,205-220$.

YATES, A.J. (1966) Psychological deficit. Ann. Rev.Psychol. 17, $111-144$.

YERKES, R.M. and DODSON, J.D. (1908) The relation of strength of stimulus to rapidity of habit formation. J.Comp. Neurol.Psychol. 18, 459-4日2.

YDUNG, L.R. (1971) Pursuit eye tracking movements. In: P. Bach-y-Rita and C.C. Collins (Eds.) The contral of eye movements. New York, Academic Press.

ZAINN, T.P. (1970) Effects of reductions in uncertainty on reaction time in schizophrenic and normal subjects. J.Exp.Res.Pers. 4, 135-143.

ZAHN, T.P. and ROSENTHAL, D. (1965) Preparatory set in acute schizophrenia. J.Nerv.Ment.Dis. 141, 352-358.

ZAHN, T.P., ROSENTHAL, D. and SHAKOW, D. (1963) Effects of irregular preparatory intervals on reaction time in schizophrenia. J.Abnorm. Peychol. 67, 44-52.

ZUBIN, J. (1975) Problem of attention in schizophrenia. In: M.L. Kietzman, 5. Sutton and J. Zubin (Eds.) Experimental approaches to psychopathology. New York, Academic Press. 
ZUBIN, J. (1981) Discussion at the WPA/APA Regional Meeting 'Critical issues in psychiatry for the $80^{\prime \prime} \mathrm{s}^{*}$, New York.

ZUBIN, J. and SPRING, B. (1977) Vulnerability. A new view of schizophrenia. J.Abnorm.Psychol. 86, 103m126. 
Geboren op 22 juli 1946 te Boskoop. HBS-B opleiding aan het Christelijk Lyceum te Alphen aan de Rijn, gevolgd door studie geneeskunde aan de Rijksuniversiteit te Leiden (1964-1970). Studenten-assistentschap fysiologie. Militaire dienstplicht vervuld bij de Sectie Geestelijke Gezondheidszorg van de Koninklijke Landmacht. Opleiding tot psychiater in de Jelgersmakliniek te Oegstgeost (opleider Prof. Dr. J. Bastiaans). Stage neurologie in de Ursulakliniek te Wassenaar; stage sociale psychiatrie bij de Stichting Geestelijke Volksgezondheid in Zuid-Holland. Psychotherapie-supervisie onder andere bij het I.M.P. in Den Haag. Sinds 1976 werkzaam als psychiater in het Psychisch Gezondheidscentrum 'Welterhof' te Heerlen, alwar ook het promotie-onderzoek werd verricht. Sinds enkele jaren bestuurslid van de Vereniging van Polikliniekmedewerkers van Psychiatrische Instellingen (V.P.P.I.). Publiceerde over obesitas, opname preventie, psychiatrische poliklinieken. 